\title{
Esquema Variacional para Estimar un Campo de Velocidad Hidrodinámico a Partir de Datos y su Aplicación Meteorológica
}

Tesis que presenta:

\author{
Gerardo Ramírez Rosario
}

para obtener el grado de Maestro en Ciencias

Maestría en Matemáticas Aplicadas e Industriales

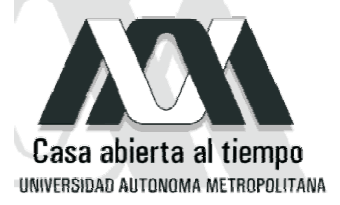

División de Ciencias Básicas e Ingeniería 



\section{Asesores}

Dr. Marco A. Núñez Peralta

Departamento de Física

Asesor

Dr. Héctor Juárez Valencia

Departamento de Matemáticas

Coasesor 



\title{
Sinodales
}

\section{Dra. María Luisa Sandoval}

Departamento de Matemáticas

\section{Dr. Gustavo Izquierdo Buenrostro}

Departamento de Matemáticas

\author{
Dr. Jorge E. Sánchez Sánchez \\ Laboratorio de simulación \\ Instituto de Matemáticas Unidad Cuernavaca \\ U.N.A.M
}





\section{Índice}

$\begin{array}{lll}\text { Introducción } & \text { IX }\end{array}$

1. Ecuaciones básicas en un sistema inercial $\quad 1$

1.1. Descripción Lagrangiana y Euleriana . . . . . . . . . . . . . . . . . . . . . . 1

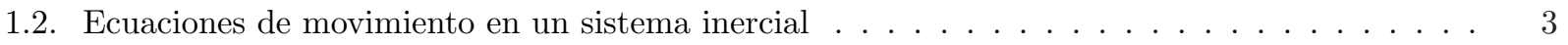

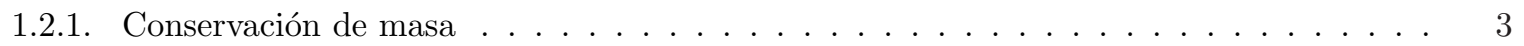

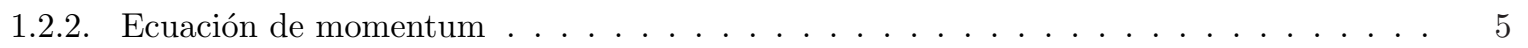

1.3. Relaciones termodinámicas, hipótesis de equilibrio local y flujo isoentrópico . . . . . . . . . 9

1.3.1. Flujo adiabático ó isoentrópico . . . . . . . . . . . . . . . . . . 11

1.3.2. Ecuaciones de Poisson . . . . . . . . . . . . . . . . . . . . . 13

1.4. Ecuaciones de Bernoulli en sistemas inerciales . . . . . . . . . . . . . . . . . . . . 14

1.4.1. Ecuación de Bernoulli para una atmósfera isotérmica . . . . . . . . . . . . . . . . 14

1.4.2. Ecuación de Bernoulli para una atmósfera adiabática . . . . . . . . . . . . . . 15

2. Ecuaciones de movimiento relativas a la tierra $\quad \mathbf{1 7}$

2.1. Ecuaciones en un sistema de referencia fijo a la Tierra . . . . . . . . . . . . . . 17

2.2. Ecuaciones relativas a un plano tangente a la esfera terrestre . . . . . . . . . . . . . . 19

2.3. Ecuaciones de momentum aproximadas y su región de validez . . . . . . . . . . . . . . 22

2.4. Ecuaciones en coordenadas curvilíneas ortogonales $s^{i}$ definidas en el sistema primario $Y^{i} \ldots 24$

2.4.1. Ecuaciones en coordenadas esféricas $\lambda, \phi, r \ldots \ldots \ldots \ldots \ldots$

2.4.2. Ecuaciones en coordenadas curvilíneas esféricas $x_{s} y_{s} z_{s} \ldots \ldots \ldots \ldots$

2.5. Ecuación de Bernoulli en un sistema fijo a la Tierra $\ldots \ldots \ldots \ldots \ldots \ldots$

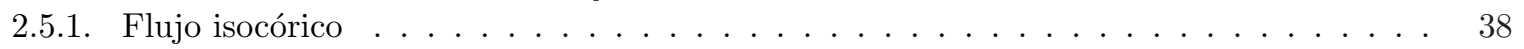

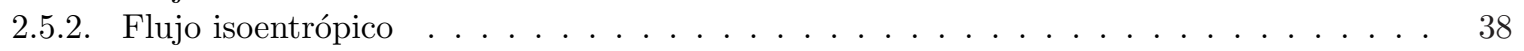

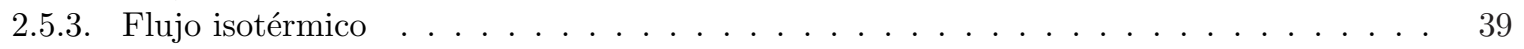

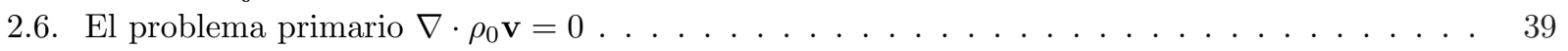

2.7. Modelo elipsoidal terrestre . . . . . . . . . . . . . . . . . . . . . . . . 40

3. Simplificación de las ecuaciones básicas 43

3.1. Análisis de escalas . . . . . . . . . . . . . . . . . . . . . 43

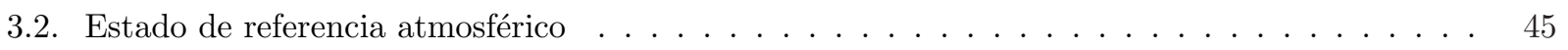

3.2.1. Existencia de un estado de referencia atmosférico . . . . . . . . . . . . . . . . 45

3.2.2. Estimación del valor de referencia $\psi^{(0)}\left(z_{s}, t\right)$ con un flujo bidimensional. . . . . . . . . 49

3.2.3. Estimación de $p^{(0)}\left(z_{s}, t\right) \ldots \ldots \ldots \ldots \ldots \ldots \ldots$

3.2.4. Región de validez de la descomposición estándar $\psi(t, \mathbf{r})=\psi_{0}(z)+\bar{\psi}(t, \mathbf{r}) \ldots \ldots \ldots$

3.2.5. Estimación del orden de magnitud del cociente $\psi_{1}(\mathbf{r}, t) / \psi_{0}(\mathbf{r}, t) \ldots \ldots \ldots \ldots$

3.2.6. Resultados con el flujo $\mathbf{v}$ sobre una topografía bidimensional . . . . . . . . . . . . 51

3.3. Simplificación de la ecuación para $w_{s} \ldots \ldots \ldots \ldots \ldots \ldots \ldots \ldots \ldots \ldots$

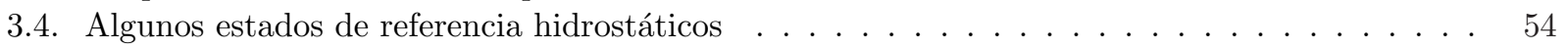

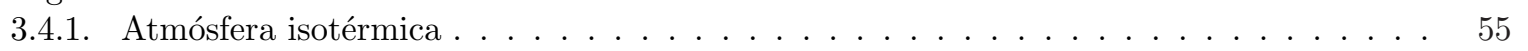

3.4.2. Atmósfera adiabática . . . . . . . . . . . . . . . . . . . . . 55

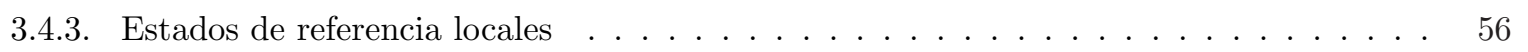


4.1. Cálculo de $\mathbf{v}(\mathbf{r})$ con el método de mapeo conforme . . . . . . . . . . . . . . . . . . . . . . 60

4.2. Interpolación Polinomial Cúbica Segmentada $\left(\mathrm{IP}^{3} \mathrm{~S}\right)$ Natural . . . . . . . . . . . . . . . . 62

4.3. Interpolación Polinomial Cúbica Segmentada Suavizada . . . . . . . . . . . . . . . . . . 63

4.4. Expresión explícita del campo v . . . . . . . . . . . . . . . . . . . . . . . 64

4.5. Algoritmo del método de mapeo conforme . . . . . . . . . . . . . . . . . . . . . . . 67

4.6. Efecto de la topografía en el campo v . . . . . . . . . . . . . . . . . . . . . 67

5. Cálculo aproximado de la solución de $\nabla \cdot \mathbf{v}=0$ a partir de un conjunto de datos experi-

mentales
5.1 . Planteamiento del problema. Campos inicial $\mathbf{v}^{0}$ y ajustado $\mathbf{v} \ldots \ldots \ldots \ldots$

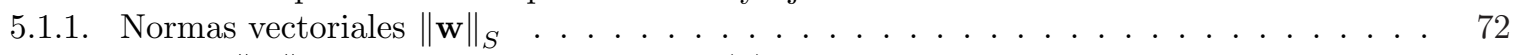

5.1.2. Norma $\|\mathbf{w}\|_{\mathcal{D} S}$ de campos vectoriales $\mathbf{w}(x) \ldots \ldots \ldots \ldots$. . . . . . . . . . . . . 74

5.2. Cálculo del campo ajustado $\mathbf{v}$ minimizando $\left\|\mathbf{w}-\mathbf{v}^{0}\right\|_{\mathcal{D S}} \operatorname{con} \nabla \cdot \mathbf{v}=0 \ldots \ldots \ldots$

5.3. Formulación variacional del problema elíptico generalizado $L_{y} \lambda=f$. . . . . . . . . . . . 79

5.4. Cálculo de $\lambda$ con el método del elemento finito . . . . . . . . . . . . . . . . . . . . . 82

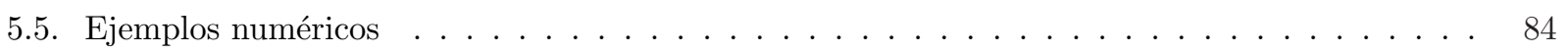

6. Formulación tensorial $\quad 95$

6.1. Base covariante $\tau_{j}$, contravariante $\eta^{j}$ y tensor métrico $\mathbb{G} \ldots \ldots . \ldots$

6.2. Forma covariante y contravariante de un vector . . . . . . . . . . . . . . . . . . . 97

6.3. Forma tensorial de $\nabla, \nabla \cdot, \mathrm{y} \nabla \cdot \rho_{0} \mathbf{v}=0 \ldots \ldots \ldots \ldots$

6.4. Cálculo del campo ajustado $\mathbf{v}$ en coordenadas generalizadas . . . . . . . . . . . . . . . . . 101

6.5. Expresiones con coordenadas $\sigma$ usadas en la literatura . . . . . . . . . . . . . . . . . 103

6.6. Formulación variacional del problema elíptico $L_{y} \lambda=f$. . . . . . . . . . . . . . . . . . . . 106

6.6.1. La condición de frontera $\partial \lambda / \partial n=0$ es incorrecta en general . . . . . . . . . . . 107

6.7. Ejemplos reportados en la literatura . . . . . . . . . . . . . . . . . . . . 108

7. Cálculo de $\mathbf{v}(\mathbf{r})$ con la restricción $\nabla \cdot \rho_{0}(\mathbf{r}) \mathbf{v}=0 \quad 115$

7.1. Cálculo en coordenadas generalizadas . . . . . . . . . . . . . . . . . . . . 115

7.1.1. Forma débil del problema elíptico asociado a $\lambda \ldots \ldots$. . . . . . . . . . . . . 117

7.1.2. Problema variacional asociado a $\lambda \ldots \ldots \ldots$. . . . . . . . . . . . . 119

7.2. Flujo alrededor de un cilindro circular . . . . . . . . . . . . . . . . . . . . . . . . . . 119

7.3. Problema de Sturm-Liouville asociado a $\lambda \ldots$. . . . . . . . . . . . . . . . . . . . . . . . . 121

7.4. Solución aproximada del problema de Sturm-Liouville con el método del elemento finito . . . 122

7.5. Cálculo del campo ajustado con elemento finito . . . . . . . . . . . . . . . . . . . . . . . 124

7.6. Resultados numéricos . . . . . . . . . . . . . . . . . . . . . . 126

$\begin{array}{ll}\text { Conclusiones y perspectivas } & 131\end{array}$

$\begin{array}{ll}\text { Bibliografía } & 135\end{array}$ 


\section{Introducción}

Hoy en día el ser humano se encuentra en el umbral de dos grandes problemas: el primero es el agotamiento de su mayor fuente de energía, el petróleo y el segundo es el cambio climático causado por el uso intensivo de éste.

Casualmente, gran parte de la solución de ambos se encuentra en el entendimiento de la dinámica atmosférica. El viento es una fuente de energía muy importante al grado que algunos países escandinavos ya han empezado a aprovecharlo para satisfacer sus requerimientos de energía eléctrica. En cuanto a la cuestión ambiental, es de todos conocido los esfuerzos a nivel mundial por revertir el calentamiento global y reducir el hoyo en la capa de ozono pues ambos fenómenos son potencialmente mortales para toda forma de vida en nuestro planeta.

Nuestro país no está exento de dichos problemas. Afortunadamente, en la cuestión energética, México tiene zonas propicias para la generación de electricidad utilizando la energía eólica y para poderla aprovechar al máximo es indispensable desarrollar modelos y técnicas capaces de caracterizar el campo de vientos en cualquier zona de una forma precisa. Por otra parte, México cuenta con una de las ciudades más pobladas del mundo: en ella se ha implementado un programa de monitoreo y control de emisión de contaminantes. Dicho programa depende de modelos que estiman la calidad del aire y de ellos depende la salud de los habitantes, de aquí la necesidad de contar con modelos confiables. Además, cada año una gran cantidad de huracanes golpean nuestras costas dejando numerosas víctimas y pérdidas materiales. Es por esto que el desarrollo de modelos que nos ayuden a comprender la dinámica de estos fenómenos es vital para la planeación de asentamientos humanos y para diseñar planes de prevención que minimicen los daños.

Los modelos de predicción numérica son la herramienta que usan los meteorólogos para calcular el estado atmosférico a diferentes lapsos de tiempo. Tales modelos realizan la predicción a partir de un estado atmosférico inicial que es construido a partir de los datos tomados de diversas fuentes como radiosondeos, radares Doppler, imágenes de satélite, etc. Los datos consisten en variables atmosféricas como la presión, temperatura, humedad, velocidad del viento, radiación solar, etc. El estado atmosférico inicial construido a partir de los datos debe cumplir con las leyes físicas que gobiernan la dinámica atmosférica, es por esta razón que debe realizarse un estudio cuidadoso de estas leyes para calcular lo mejor posible dicho estado.

La presente tesis es una introducción al análisis de datos atmosféricos cuyo principal objetivo es la construcción, a partir de datos, de un campo de viento que satisfaga la ecuación de continuidad. Sin embargo, también se hace una revisión de algunos aspectos físicos involucrados en la dinámica atmosférica.

En el Capítulo 1 veremos las ecuaciones básicas que determinan el comportamiento de un fluido ideal en un sistema de referencia inercial, así como la termodinámica básica que relaciona diferentes variables atmosféricas como la presión, la temperatura y la densidad.

En el Capítulo 2 extenderemos los conceptos del Capítulo 1 a sistemas de referencia no inerciales haciendo principal énfasis en el modelo esférico terrestre. También se calcula la región de validez de las ecuaciones de momentum utilizadas en algunos textos recientes de meteorología. Además, se hace un breve comentario acerca de el modelo elipsoidal; haciendo notar al lector que el modelo esférico terrestre es de carácter local, es decir, el modelo esférico terrestre no puede usarse para desarrollar modelos globales.

Dada la complejidad de las ecuaciones obtenidas en el Capítulo 2, en el Capítulo 3 se discute la simplificación de tales ecuaciones mediante la definición de un estado de referencia atmosférico. También se dan ejemplos de algunas atmósferas de referencia utilizando los conceptos desarrollados en los capítulos anteriores.

Desgraciadamente, el campo de viento proporcionado por las redes de monitoreo ambiental no cuenta con los datos suficientes que nos permitan utilizarlo para verificar, de manera confiable, los resultados presentados en este trabajo. Además, dicho campo tiene el inconveniente de carecer de la componente vertical, es decir, 
las redes de monitoreo sólo reportan la componente horizontal del campo de viento, lo cual dificulta, aún más, la validación de los resultados obtenidos con los diversos métodos de interpolación. Es por esto que en el Capítulo 4 se desarrolla un método analítico para obtener un campo de viento bidimensional que satisface la ecuación de continuidad. Este campo, además de proveernos de datos sintéticos que nos evitan la penosa tarea de lidiar con datos obtenidos de las redes de monitoreo, nos proporciona un campo de viento con el cual podemos verificar de manera precisa la bondad de los esquemas de interpolación.

Una vez obtenidas las características más importantes de la atmósfera podemos, con toda confianza, plantear el esquema variacional que se propone para ajustar un campo de viento con divergencia cero a los datos obtenidos con el método analítico descrito en el capítulo anterior. En el Capítulo 5 se plantea teóricamente tal esquema variacional para sistemas cartesianos; además, se utiliza la hipótesis de que la densidad de la atmósfera es constante y se dan algunos ejemplos numéricos.

En el ambiente meteorológico, los sistemas cartesianos no son los únicos que se utilizan. También son utilizados sistemas curvilíneos que no son ortogonales, por ejemplo, las coordenadas $\sigma$ que son coordenadas que siguen el terreno, o las coordenadas de presión, entre otros. El Capítulo 6 está dedicado a la extensión de la teoría del capítulo 5 a este tipo de sistemas.

Para finalizar, en el Capítulo 7 se replantea el esquema desarrollado en el capítulo 5 para una atmósfera con densidad variable. Se dan algunos ejemplos numéricos que muestran las diferencias que hay entre suponer la densidad constante y suponerla variable. Además, se da un desarrollo detallado de la solución del problema elíptico asociado al funcional que minimiza la distancia entre los datos y el campo interpolado. 


\section{Capítulo 1}

\section{Ecuaciones básicas en un sistema inercial}

El objetivo del presente capítulo es obtener correctamente las ecuaciones de movimiento de una partícula de fluido respecto a un sistema de referencia inercial así como presentar las relaciones termodinámicas que mantienen las variables de estado de la atmósfera.

\subsection{Descripción Lagrangiana y Euleriana}

La meteorología aprovecha las propiedades hidrodinámicas y termodinámicas que la atmósfera tiene para el estudio de los fenómenos que en ella se llevan a cabo. La propiedad que sirve de base para el desarrollo de modelos teóricos es la posición de algún elemento de volumen infinitesimal de fluido, llamado parcela, respecto de algún sistema cartesiano inercial. Si en un instante $t$ una parcela de aire tiene coordenadas $X^{i}$, su vector de posición está dado por

$$
\mathbf{R}(t)=X^{i} \hat{\mathbf{X}}_{i} \quad i=1,2,3
$$

donde $\hat{\mathbf{X}}_{i}$ son los vectores base del sistema cartesiano inercial. Ya que el fluido está compuesto por muchas parcelas es necesario etiquetarlas para distinguirlas entre sí con su posición inicial $\left(t=t_{0}\right)$. Es por esto que las coordenadas instantáneas de una parcela en particular dependen tanto de su posición inicial como del tiempo

$$
X^{i}=X^{i}\left(X_{0}^{j}, t\right)
$$

Esta relación entre las coordenadas instantáneas e iniciales es única para cada partícula pues no puede haber dos parcelas distintas en un mismo lugar al mismo tiempo. Se acostumbra llamar a las coordenadas instantáneas "coordenadas Eulerianas" y a las coordenadas iniciales "coordenadas Lagrangianas". Ya que la relación es única podemos obtener la relación inversa de (1.2), la que nos da la expresión de las coordenadas Lagrangianas en términos de las Eulerianas

$$
X_{0}^{i}=X_{0}^{i}\left(X^{j}, t\right) .
$$

A pesar de que las relaciones (1.2) y (1.3) pueden ser no lineales, podemos asociar localmente una transformación $\mathbb{T}$ tal que

$$
\begin{gathered}
\mathbf{X}=X\left(t, X_{0}\right)=\mathbb{T}\left(\mathbf{X}_{0}\right) \\
\mathbf{X}_{0}=X_{0}(t, \mathbf{X})=\mathbb{T}^{-1}(\mathbf{X})
\end{gathered}
$$

Para calcular la velocidad de una parcela de aire se aprovecha que los vectores base $\hat{\mathbf{X}}^{i}$ son constantes. Así que el vector velocidad es

$$
\frac{d \mathbf{R}(t)}{d t}=\frac{d X^{i}\left(X_{0}^{j}, t\right)}{d t} \hat{\mathbf{X}}_{i}=V_{L}\left(X_{0}^{j}, t\right) \hat{\mathbf{X}}_{i}
$$


Las componentes de la velocidad $V^{i}\left(X_{0}^{j}, t\right)$ sólo dependen de las coordenadas Lagrangianas, por lo que se les conoce por componentes Lagrangianas de la velocidad. Para obtener la expresión Euleriana de la velocidad se usa la ecuación (1.3) en (1.5)

$$
V_{E}^{i}\left(X^{i}, t\right)=V_{L}^{i}\left(X_{0}^{j}\left(X^{k}, t\right), t\right)
$$

Para calcular la aceleración usamos (1.6)

$$
A^{i}(X, t)=\frac{d V^{i}\left(X^{j}, t\right)}{d t}
$$

Usando la regla de la cadena

$$
\frac{d V^{i}\left(X^{j}, t\right)}{d t}=\left(\frac{\partial V^{i}\left(X^{k}, t\right)}{\partial t}\right)_{\mathbf{X}=\mathbf{c t e}}+\frac{d X^{j}}{d t} \frac{\partial V^{i}\left(X^{k}, t\right)}{\partial X^{j}}
$$

Recordemos que $\frac{d X^{j}}{d t}$ puede escribirse tanto en coordenadas Eulerianas como en coordenadas Lagrangianas dando por resultado expresiones equivalentes para las componentes de la aceleración

$$
\begin{aligned}
& \frac{d V^{i}\left(X^{j}, t\right)}{d t}=\left(\frac{\partial V^{i}\left(X^{k}, t\right)}{\partial t}\right)_{\mathbf{X}=\mathbf{c t e} .}+V_{L}^{j}\left(X_{0}^{k}, t\right) \frac{\partial V^{i}\left(X^{k}, t\right)}{\partial X^{j}} \\
& \frac{d V^{i}\left(X^{j}, t\right)}{d t}=\left(\frac{\partial V^{i}\left(X^{k}, t\right)}{\partial t}\right)_{\mathbf{X}=\mathbf{c t e} .}+V_{E}^{j}\left(X^{k}, t\right) \frac{\partial V^{i}\left(X^{k}, t\right)}{\partial X^{j}} .
\end{aligned}
$$

Para simplificar la notación se introduce el vector gradiente

$$
\nabla_{X}=\mathbf{X}_{i} \frac{\partial}{\partial X^{i}}
$$

entonces las componentes de la aceleración quedan como sigue

$$
\begin{aligned}
& A^{i}\left(X^{k}, t\right)=\left(\frac{\partial V^{i}\left(X^{k}, t\right)}{\partial t}\right)_{\mathbf{X}=\mathbf{c t e} .}+\mathbf{V}_{L} \cdot \nabla_{X} V^{i} \\
& A^{i}\left(X^{k}, t\right)=\left(\frac{\partial V^{i}\left(X^{k}, t\right)}{\partial t}\right)_{\mathbf{X}=\mathbf{c t e} .}+\mathbf{V}_{E} \cdot \nabla_{X} V^{i} .
\end{aligned}
$$

Aparte de conocer la posición, velocidad y aceleración de una parcela, también nos interesa la distribución espacial y temporal de otras propiedades como la presión o la temperatura. Ya que la posición puede darse en coordenadas Eulerianas y en coordenadas Lagrangianas, una propiedad $\mathbb{Q}$ puede expresarse en términos de estos dos sistemas

$$
\begin{aligned}
\mathcal{Q}_{E} & \equiv \mathcal{Q}\left(t, X^{i}\right) \\
\mathcal{Q}_{L} & \equiv \mathcal{Q}\left(t, X_{0}^{i}\right)
\end{aligned}
$$

A pesar de que las ecuaciones (1.9) y (1.10) tienen expresiones diferentes el valor de $\mathbb{Q}$ debe ser el mismo tratándose de la misma parcela, por lo que

$$
\mathcal{Q}_{L}\left(t, X_{0}^{i}\right)=\mathcal{Q}_{E}\left(t, X^{j}\right)
$$

donde $X$ y $X_{0}$ están relacionados por (1.2). El cambio de la propiedad $\mathbb{Q}$ en el tiempo es

$$
\frac{d \mathcal{Q}_{L}\left(t, X_{0}^{i}\right)}{d t}=\frac{d \mathcal{Q}_{E}\left(t, X^{j}\right)}{d t}
$$


como las componentes del vector $\mathbf{X}$ dependen del tiempo, entonces

$$
\begin{aligned}
& \frac{d \mathcal{Q}_{L}\left(t, X_{0}^{i}\right)}{d t}=\left(\frac{\partial \mathcal{Q}_{E}}{\partial t}\right)_{\mathbf{X}=\mathbf{c t e} .}+\frac{\partial X^{j}}{\partial t} \frac{\partial \mathcal{Q}_{E}}{\partial X^{j}} \\
& \frac{d \mathcal{Q}_{L}\left(t, X_{0}^{i}\right)}{d t}=\left(\frac{\partial \mathcal{Q}_{E}}{\partial t}\right)_{\mathbf{X}=\mathbf{c t e} .}+\mathbf{V} \cdot \nabla \mathcal{Q}_{E}
\end{aligned}
$$

Al término del lado izquierdo se le conoce como derivada material y representa el cambio de la propiedad $\mathbb{Q}$ en el interior de la parcela que en $t_{0}$ estaba en $X_{0}$. El primer miembro del lado derecho representa el cambio de $\mathbb{Q}$ en un punto fijo y el segundo miembro mide el cambio de $\mathbb{Q}$ debido al movimiento de la parcela y a la distribución espacial de $\mathbb{Q}$.

\subsection{Ecuaciones de movimiento en un sistema inercial}

Una vez establecida la manera de calcular la variación temporal de alguna propiedad $\mathbb{Q}$ dedicaremos la presente sección al estudio de tres propiedades muy importantes: la masa, el momentum y la energía de una parcela de aire encerrada en un volumen arbitrario $v$. Dicho volumen debe encerrar las mismas partículas mientras se mueve con el fluido, por lo que éste cambia su forma en cada instante.

\subsubsection{Conservación de masa}

La cantidad de masa en un volumen constituido por las mismas partículas en coordenadas Eulerianas (CE) es

$$
M(t)=\int_{v(t)} \rho(\mathbf{X}, t) d v(t)
$$

donde $\rho(\mathbf{X}, t)$ es la densidad en CE. El cambio temporal de la masa está dado por

$$
\frac{d M(t)}{d t}=\frac{d}{d t} \int_{v(t)} \rho(\mathbf{X}, t) d v(t)
$$

Como la región de integración cambia con el tiempo conviene expresar todo en términos de una integral sobre una región independiente del tiempo. Esto se puede hacer reemplazando las CE por las CL usando la relación (1.2). Este cambio de variable transforma la región $v(t)$ por aquella $v\left(t_{0}\right)$ que ocupaban las partículas en el instante $t_{0}$ y la integral se transforma como sigue

$$
\int_{v(t)} \rho(\mathbf{X}, t) d v(t)=\int_{v\left(t_{0}\right)} \rho\left(\mathbf{X}_{0}, t\right) J d v\left(t_{0}\right)
$$

donde $J$ es el determinante de la matriz Jacobiana asociada a la transformación (1.4). Entonces tenemos

$$
\frac{d M(t)}{d t}=\int_{v\left(t_{0}\right)} \frac{d}{d t}\left[\rho\left(\mathbf{X}_{0}, t\right) J\right] d v\left(t_{0}\right)=\int_{v\left(t_{0}\right)}\left[\frac{d \rho\left(\mathbf{X}_{0}, t\right)}{d t} J+\rho\left(\mathbf{X}_{0}, t\right) \frac{d J}{d t}\right] d v\left(t_{0}\right)
$$

De aquí en adelante utilizaremos la notación de índices repetidos

$$
a_{i j} b_{j k}=a_{i 1} b_{1 k}+a_{i 2} b_{2 k}+a_{i 3} b_{3 k}
$$

para hacer más compactas las expresiones. Con dicha notación el Jacobiano es

$$
J=\epsilon_{i j k} \frac{\partial X^{1}}{\partial X_{i}^{0}} \frac{\partial X^{2}}{\partial X_{j}^{0}} \frac{\partial X^{3}}{\partial X_{k}^{0}}
$$

donde $\epsilon_{i j k}$ es el símbolo de Levi-Civita cuya definición es la siguiente:

$$
\epsilon_{i j k}=\left\{\begin{array}{ccc}
1 & \text { si } & i j k \text { es una permutación par } \\
-1 & \text { si } & i j k \text { es una permutación impar } \\
0 & & \text { si dos índices se repiten }
\end{array} .\right.
$$


Derivando el Jacobiano respecto al tiempo obtenemos

$$
\frac{d J}{d t}=\epsilon_{i j k} \frac{d}{d t}\left[\frac{\partial X^{1}}{\partial X_{i}^{0}} \frac{\partial X^{2}}{\partial X_{j}^{0}} \frac{\partial X^{3}}{\partial X_{k}^{0}}\right]=\epsilon_{i j k}\left\{\frac{\partial \dot{X}^{1}}{\partial X_{i}^{0}} \frac{\partial X^{2}}{\partial X_{j}^{0}} \frac{\partial X^{3}}{\partial X_{k}^{0}}+\frac{\partial X^{1}}{\partial X_{i}^{0}} \frac{\partial \dot{X}^{2}}{\partial X_{j}^{0}} \frac{\partial X^{3}}{\partial X_{k}^{0}}+\frac{\partial X_{1}}{\partial X_{i}^{0}} \frac{\partial X^{2}}{\partial X_{j}^{0}} \frac{\partial \dot{X}^{3}}{\partial X_{k}^{0}}\right\}
$$

donde hemos supuesto que las derivadas temporal y espacial se pueden intercambiar. Por otra parte, las velocidades $\dot{x}_{m}$ son funciones de las coordenadas eulerianas $x_{l}$ y éstas, a su vez, son función de las coordenadas Lagrangianas $x_{n}^{0}$, entonces, podemos usar la regla de la cadena para intentar expresar la derivada temporal de Jacobiano en términos del mismo Jacobiano. Así que, por la regla de la cadena

$$
\frac{\partial \dot{X}^{m}}{\partial X_{n}^{0}}=\frac{\partial X^{l}}{\partial X_{n}^{0}} \frac{\partial \dot{X}^{m}}{\partial X_{l}}
$$

tenemos que la derivada temporal del Jacobiano en términos del mismo Jacobiano es

$$
\begin{aligned}
\frac{d J}{d t} & =\epsilon_{i j k}\left\{\frac{\partial X^{l}}{\partial X_{i}^{0}} \frac{\partial X^{2}}{\partial X_{j}^{0}} \frac{\partial X^{3}}{\partial X_{k}^{0}} \frac{\partial \dot{X}^{1}}{\partial X_{l}}+\frac{\partial X^{1}}{\partial X_{i}^{0}} \frac{\partial X^{l}}{\partial X_{j}^{0}} \frac{\partial X^{3}}{\partial X_{k}^{0}} \frac{\partial \dot{X}^{2}}{\partial X_{l}}+\frac{\partial X^{1}}{\partial X_{i}^{0}} \frac{\partial X^{2}}{\partial X_{j}^{0}} \frac{\partial X^{l}}{\partial X_{k}^{0}} \frac{\partial \dot{X}^{3}}{\partial X_{l}}\right\} \\
& =\delta_{1 l} J \frac{\partial \dot{X}^{1}}{\partial X_{l}}+\delta_{2 l} J \frac{\partial \dot{X}^{2}}{\partial X_{l}}+\delta_{3 l} J \frac{\partial \dot{X}^{3}}{\partial X_{l}} \\
& =J \nabla \cdot \mathbf{V} .
\end{aligned}
$$

Sustituyendo esta última expresión en la integral anterior

$$
\frac{d M}{d t}=\int_{v\left(t_{0}\right)}\left[\frac{d \rho\left(\mathbf{X}_{0}, t\right)}{d t}+\rho\left(\mathbf{X}_{0}, t\right) \nabla \cdot \mathbf{V}\right] J d v\left(t_{0}\right)
$$

Regresando a las CE, la expresión del cambio temporal de la masa dentro de algún volumen es

$$
\frac{d M}{d t}=\int_{v(t)}\left[\frac{d \rho(\mathbf{X}, t)}{d t}+\rho(\mathbf{X}, t) \nabla \cdot \mathbf{V}\right] d v(t)
$$

Usando (1.12) tenemos

$$
\frac{d \rho(\mathbf{X}, t)}{d t}=\frac{\partial \rho(\mathbf{X}, t)}{\partial t}+\mathbf{V} \cdot \nabla \rho
$$

entonces

$$
\frac{d M}{d t}=\int_{v(t)}\left[\frac{\partial \rho(\mathbf{X}, t)}{\partial t}+\nabla \cdot \rho(\mathbf{X}, t) \mathbf{V}\right] d v(t)
$$

Este procedimiento puede aplicarse a cualquier función $\rho \mathcal{A}$ para obtener el Teorema de Transporte de Reynolds

$$
\frac{d}{d t} \int_{v(t)} \rho \mathcal{A} d \Omega(t)=\int_{v(t)}\left[\frac{\partial \rho \mathcal{A}}{\partial t}+\nabla \cdot(\rho \mathcal{A} \mathbf{V})\right] d v(t)
$$

Regresando a nuestro caso, recordemos que el volumen que estamos considerando encierra a las mismas partículas, por lo que el cambio de la masa del volumen en el tiempo es nulo

$$
\int_{v(t)}\left[\frac{\partial \rho(\mathbf{X}, t)}{\partial t}+\nabla \cdot \rho(\mathbf{X}, t) \mathbf{V}\right] d v(t)=0 .
$$

Como esta relación debe cumplirse para cualquier volumen, entonces se debe cumplir que

$$
\frac{\partial \rho(\mathbf{X}, t)}{\partial t}+\nabla \cdot \rho(\mathbf{X}, t) \mathbf{V}=0 \quad \text { ó } \quad \frac{d \rho(\mathbf{X}, t)}{d t}+\rho(\mathbf{X}, t) \nabla \cdot \mathbf{V}=0
$$

Esta ecuación es conocida como la ecuación de continuidad ó ecuación de conservación de masa. 


\subsubsection{Ecuación de momentum}

El momentum de un conjunto de partículas encerradas en un volumen $v(t)$ está dado por

$$
\mathbf{p}(t)=\int_{v(t)} \mathbf{V} \rho d v(t) .
$$

La segunda ley de Newton establece que la derivada temporal de (1.17) es igual a la fuerza resultante que actúa sobre el volumen $v(t)$

$$
\frac{d \mathbf{p}(t)}{d t}=\frac{d}{d t} \int_{v(t)} \mathbf{V} \rho d v(t) .
$$

Las fuerzas que actúan sobre un fluido pueden clasificarse en dos tipos: fuerzas de largo alcance ó volumétricas, que afectan a todas las partículas dentro del volumen y fuerzas de corto alcance, ó de superficie, que se producen por la interacción entre las partículas que están en la superficie del volumen y las partículas del resto del fluido que se localizan en la superficie que encierra al volumen. Las fuerzas de volumen pueden expresarse como

$$
F_{v o l}=\int_{v(t)} \mathbf{f}(X, t) \rho d v(t)
$$

donde $\mathbf{f}(X, t)$ es la fuerza por unidad de masa. Las fuerzas superficiales se pueden cuantificar con

$$
F_{\text {sup }}=\oint_{S(t)} \mathbf{t}^{(n)}(X, t) d S(t)
$$

donde $\mathbf{t}^{(n)}$ es el vector normal del tensor y el superíndice $n$ indica que ésta fuerza superficial sólo depende de la componente normal a la superficie $d S(t)$. Para visualizar la expresión del vector de tensión se han escogido los tres planos cartesianos para escribir los tres vectores de tensión asociados a la base canónica (ver figura 1.2 y figura 1.3$)$.

$$
\begin{aligned}
& \mathbf{t}^{\left(e_{1}\right)}=t_{1}^{\left(e_{1}\right)} \hat{\mathbf{e}}_{1}+t_{2}^{\left(e_{1}\right)} \hat{\mathbf{e}}_{2}+t_{3}^{\left(e_{1}\right)} \hat{\mathbf{e}}_{3} \\
& \mathbf{t}^{\left(e_{2}\right)}=t_{1}^{\left(e_{2}\right)} \hat{\mathbf{e}}_{1}+t_{2}^{\left(e_{2}\right)} \hat{\mathbf{e}}_{2}+t_{3}^{\left(e_{2}\right)} \hat{\mathbf{e}}_{3} \\
& \mathbf{t}^{\left(e_{3}\right)}=t_{1}^{\left(e_{3}\right)} \hat{\mathbf{e}}_{1}+t_{2}^{\left(e_{3}\right)} \hat{\mathbf{e}}_{2}+t_{3}^{\left(e_{3}\right)} \hat{\mathbf{e}}_{3}
\end{aligned}
$$

o en forma matricial

$$
\left[\begin{array}{l}
\mathbf{t}^{\left(e_{1}\right)} \\
\mathbf{t}^{\left(e_{2}\right)} \\
\mathbf{t}^{\left(e_{3}\right)}
\end{array}\right]=\left[\begin{array}{lll}
t_{1}^{\left(e_{1}\right)} & t_{2}^{\left(e_{1}\right)} & t_{3}^{\left(e_{1}\right)} \\
t_{1}^{\left(e_{2}\right)} & t_{2}^{\left(e_{2}\right)} & t_{3}^{\left(e_{2}\right)} \\
t_{1}^{\left(e_{3}\right)} & t_{2}^{\left(e_{3}\right)} & t_{3}^{\left(e_{3}\right)}
\end{array}\right]\left[\begin{array}{c}
\hat{\mathbf{e}}_{1} \\
\hat{\mathbf{e}}_{2} \\
\hat{\mathbf{e}}_{3}
\end{array}\right]
$$

donde la matriz $3 \times 3$ se le conoce como el tensor de tensión. Otra forma de escribir dicha matriz es

$$
\Sigma=\left[\begin{array}{lll}
\sigma_{11} & \sigma_{12} & \sigma_{13} \\
\sigma_{21} & \sigma_{22} & \sigma_{23} \\
\sigma_{31} & \sigma_{32} & \sigma_{33}
\end{array}\right]
$$

Para obtener la expresión del vector de tensión asociado a un plano arbitrario se realiza un balance de fuerzas en el interior de un tetraedro elemental de fluido que tiene su vértice en el punto $P$ y su base normal a la superficie arbitraria, como muestra la figura

Si suponemos que el tetraedro está en equilibrio, el balance de fuerzas está dado por

$$
\mathbf{t}^{(n)} d A-\mathbf{t}^{(1)} d A_{1}-\mathbf{t}^{(2)} d A_{2}-\mathbf{t}^{(3)} d A_{3}+\rho \mathbf{f} d v=0
$$

Las superficies están relacionadas por proyección

$$
d A_{i}=n_{i} d A
$$

donde $n_{i}$ son las componentes del vector normal a la superficie arbitraria. El volumen del tetraedro está dado por

$$
d V=\frac{1}{3} h d A
$$




\section{$4 x^{3}$}

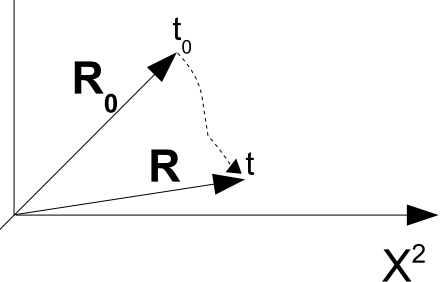

\section{$-x^{1}$}

Figura 1.1: Sistema de referencia inercial $X^{i}$.

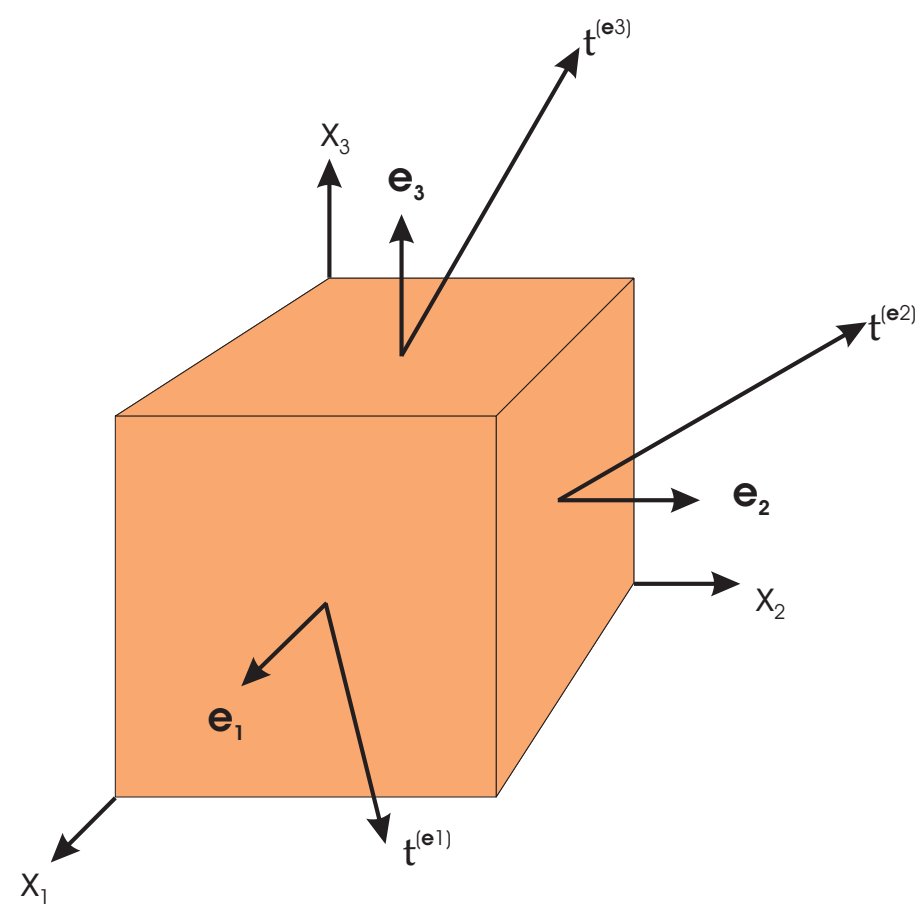

Figura 1.2: Vectores de tensión asociados a los planos cartesianos 


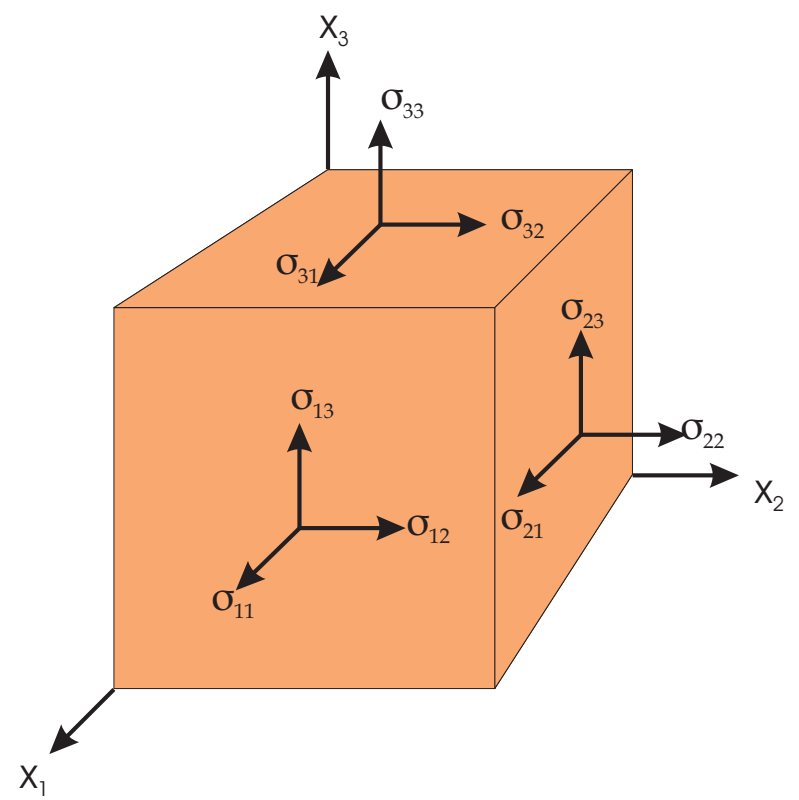

Figura 1.3: Componentes de los vectores de tensión sobre las caras del cubo

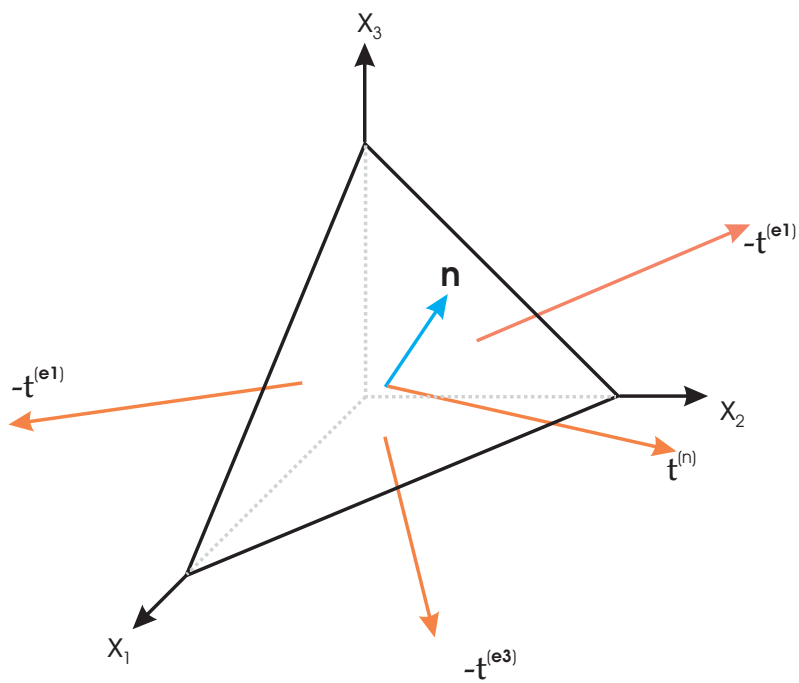

Figura 1.4: Vector de tensión asociado a un plano arbitrario. 
donde $h$ es la altura del tetraedro. Entonces, el balance de fuerzas da

$$
\left[\mathbf{t}^{(n)}-\mathbf{t}^{\left(e_{1}\right)} n_{1}-\mathbf{t}^{\left(e_{2}\right)} n_{2}-\mathbf{t}^{\left(e_{3}\right)} n_{3}+\frac{\rho}{3} \mathbf{f} h\right] d A=0
$$

y por tratarse de una superficie arbitraria

$$
\mathbf{t}^{(n)}-\mathbf{t}^{\left(e_{1}\right)} n_{1}-\mathbf{t}^{\left(e_{2}\right)} n_{2}-\mathbf{t}^{\left(e_{3}\right)} n_{3}+\frac{\rho}{3} \mathbf{f} h=0
$$

si hacemos tender la altura a cero $(h \rightarrow 0)$ obtenemos la relación entre el vector de tensión asociado a una superficie arbitraria en términos de los vectores de tensión cartesianos

$$
\mathbf{t}^{(n)}=\mathbf{t}^{(1)} n_{1}+\mathbf{t}^{(2)} n_{2}+\mathbf{t}^{(3)} n_{3} .
$$

En forma matricial

$$
\left[\begin{array}{lll}
t_{1}^{(n)} & t_{2}^{(n)} & t_{3}^{(n)}
\end{array}\right]=n_{1}\left[\begin{array}{l}
t_{1}^{\left(e_{1}\right)} \\
t_{2}^{\left(e_{1}\right)} \\
t_{3}^{\left(e_{1}\right)}
\end{array}\right]+n_{2}\left[\begin{array}{l}
t_{1}^{\left(e_{2}\right)} \\
t_{2}^{\left(e_{2}\right)} \\
t_{3}^{\left(e_{2}\right)}
\end{array}\right]+n_{3}\left[\begin{array}{l}
t_{1}^{\left(e_{3}\right)} \\
t_{2}^{\left(e_{3}\right)} \\
t_{3}^{\left(e_{3}\right)}
\end{array}\right]=\left[\begin{array}{lll}
n_{1} & n_{2} & n_{3}
\end{array}\right]\left[\begin{array}{lll}
\sigma_{11} & \sigma_{12} & \sigma_{13} \\
\sigma_{21} & \sigma_{22} & \sigma_{23} \\
\sigma_{31} & \sigma_{32} & \sigma_{33}
\end{array}\right]
$$

o simplemente

$$
\mathbf{t}^{(n)}=\mathbf{n}^{T} \boldsymbol{\Sigma} .
$$

En el caso de fluidos no viscosos, como la atmósfera, la matriz $\Sigma$ es diagonal y sus elementos no nulos $\left(\Sigma_{i i}=-p\right)$ están ocupados por la presión atmosférica y ésta actúa siempre normal a cualquier superficie sin importar su orientación, lo que quiere decir que

$$
\mathbf{t}^{(n)}=-p \hat{\mathbf{n}}
$$

donde $\hat{\mathbf{n}}$ es el vector normal unitario a la superficie del volumen $\Omega(t)$. Sustituyendo en (1.30) tenemos

$$
F_{\text {sup }}=\oint_{S(t)}-p \hat{\mathbf{n}} d S(t)
$$

Igualando (1.18) con la suma de (1.19) y (1.20)

$$
\frac{d}{d t} \int_{v(t)} \mathbf{V} \rho d v(t)=\oint_{S(t)}-p \hat{\mathbf{n}} d S(t)+\int_{\Omega(t)} \mathbf{f}(X, t) \rho d v(t) .
$$

Usando (1.15) en el lado izquierdo

$$
\frac{d}{d t} \int_{v(t)} \mathbf{V} \rho d v(t)=\int_{v(t)}\left[\frac{d(\mathbf{V} \rho)}{d t}+\mathbf{V} \rho \nabla \cdot \mathbf{V}\right] d v(t)
$$

Desarrollando

$$
\int_{v(t)}\left[\frac{d(\mathbf{V} \rho)}{d t}+\mathbf{V} \rho \nabla \cdot \mathbf{V}\right] d v(t)=\int_{v(t)}[\rho \frac{d \mathbf{V}}{d t}+\underbrace{\left(\frac{d \rho}{d t}+\rho \nabla \cdot \mathbf{V}\right)}_{\text {Ec. de continuidad }}] d v(t)=\int_{v(t)} \rho \frac{d \mathbf{V}}{d t} d v(t)
$$

Donde hemos usado (1.16). Sustituyendo en (1.22)

$$
\int_{v(t)} \rho \frac{d \mathbf{V}}{d t} d v(t)=\oint_{S(t)}-p \hat{\mathbf{n}} d S+\int_{v(t)} \mathbf{f}(X, t) \rho d v(t) .
$$

Por el teorema de la divergencia

$$
\oint_{S(t)} p \hat{\mathbf{n}} d S=\int_{v(t)} \nabla p d v(t)
$$


entonces

$$
\int_{v(t)}\left[\rho \frac{d \mathbf{V}}{d t}+\nabla p-\mathbf{f}(X, t) \rho\right] d v(t)=0,
$$

ecuación válida para un volumen arbitrario, por lo que

$$
\frac{d \mathbf{V}}{d t}+\frac{1}{\rho} \nabla p-\mathbf{f}(X, t)=0 .
$$

A esta ecuación se le conoce como ecuación de momentum.

\subsection{Relaciones termodinámicas, hipótesis de equilibrio local y flu- jo isoentrópico}

La primera ley de la termodinámica establece la conservación de la energía

$$
d U=d Q+d W
$$

para procesos reversibles o irreversibles, donde la energía interna $U$ es un atributo de cada sistema físico, $d Q$ es el calor que fluye a través de las fronteras del sistema y $d W$ es el trabajo realizado por el sistema. En el caso de un gas ideal como la atmósfera tenemos

$$
d W=-p d v
$$

donde suponemos que la presión $p$ es la misma en cada punto del gas. La segunda ley de la termodinámica establece que para procesos reversibles tiene lugar la relación

$$
d U=T d S-p d v
$$

donde $S$ es la entropía del sistema. Esta relación supone que el sistema está cerca del equilibrio termodinámico, por lo que no hay diferencias significativas entre los valores de sus propiedades termodinámicas en distintos puntos del sistema. Sin embargo, las propiedades de un fluido en movimiento pueden cambiar significativamente de valor de un punto a otro por lo que, en general, el fluido constituye un sistema fuera de equilibrio termodinámico y, en consecuencia, la relación termodinámica (1.25) no es válida. Podemos evitar esta dificultad si consideramos que nuestro (micro) sistema termodinámico es una partícula de fluido y, por su pequeñez, suponemos que dicho sistema está básicamente en equilibrio termodinámico. Esta hipótesis recibe el nombre de hipótesis del equilibrio local. De acuerdo con esta hipótesis el valor de las variables termodinámicas pueden diferir de una partícula a otra por lo que ahora debemos considerarlas funciones de la posición de cada partícula. Por ejemplo, si usamos el subíndice 0 para denotar el valor de una propiedad como función de las coordenadas lagrangianas de cada partícula, tenemos

$$
U_{0}=U_{0}\left(t, X_{0}\right), \quad S_{0}=S_{0}\left(t, X_{0}\right), \quad T_{0}=T_{0}\left(t, X_{0}\right), \quad \text { etc. },
$$

y la segunda ley (1.14) toma la forma

$$
d U_{0}=T_{0} d S_{0}-p_{0} d v_{0}
$$

Si las propiedades termodinámicas son funciones bien comportadas del tiempo $t$ de manera que las derivadas respecto a $t$ están bien definidas, las diferenciales en (1.27) están dadas por

$$
d U_{0}=\frac{d U_{0}}{d t} d t, \quad d S_{0}=\frac{d S_{0}}{d t} d t, \quad d v_{0}=\frac{d v_{0}}{d t} d t
$$

y la segunda ley en su forma (1.27) equivale a la relación siguiente

$$
\frac{d U_{0}}{d t}=T_{0} \frac{d S_{0}}{d t}-p_{0} \frac{d V_{0}}{d t}
$$

Para reescribir lo anterior en forma euleriana basta con sustituir las coordenadas lagrangianas $X_{0}$ por su expresión en coordenadas eulerianas (1.4), por ejemplo

$$
U(t, X) \equiv U_{0}\left(t, X_{0}=X_{0}(t, X)\right)
$$


donde omitimos el subíndice o para indicar a una propiedad en su forma euleriana. Así, la relación termodinámica (1.27) toma la forma

$$
d U=T d S-p d V,
$$

donde usamos el hecho de que cada función depende de $t$ y $X^{i}$ para obtener la expresión de las diferenciales. Por ejemplo, para la energía interna tenemos

$$
d U=\frac{\partial U}{\partial t} d t+\frac{\partial U}{\partial X^{i}} d X^{i} .
$$

De acuerdo con la definición de $\nabla$ y la relación (1.1), podemos escribir

$$
\frac{\partial U}{\partial X^{i}} d X^{i}=\nabla U \cdot d \mathbf{R}
$$

con lo cual llegamos a la expresión

$$
d U=\frac{\partial U}{\partial t} d t+\nabla U \cdot d \mathbf{R}
$$

válida para flujos no estacionarios. Si el flujo es estacionario tenemos

$$
d U=\nabla U \cdot d \mathbf{R}
$$

Reescribamos (1.29) en términos de la densidad

$$
\rho=\frac{M}{v}=\frac{\text { masa }}{\text { volumen }}
$$

donde la masa $M$ de cada partícula de fluido se considera constante. Entonces

$$
d v=d \frac{M}{\rho}=-\frac{M}{\rho^{2}} d \rho .
$$

Sustituyendo en (1.29) y dividiendo entre $M$ se obtiene

$$
d\left(\frac{U}{M}\right)=T d\left(\frac{S}{M}\right)+\frac{p}{\rho} d \rho .
$$

Si definimos

la segunda ley (1.29) toma la forma

$$
u \equiv \frac{U}{M} \quad s \equiv \frac{S}{M}
$$

$$
d u=T d s+\frac{p}{\rho^{2}} d \rho .
$$

Reescribamos esta última ecuación en términos de la función de entalpía

Dividiendo entre $M$,

$$
H \equiv U+p V=U+p \frac{M}{\rho} .
$$

y definiendo

$$
\frac{H}{M}=\frac{U}{M}+\frac{p}{\rho},
$$

se obtiene

$$
h \equiv \frac{H}{M}
$$

y por tanto

$$
h=u+\frac{p}{\rho}
$$

$$
d h=d u+\frac{d p}{\rho}-\frac{p}{\rho^{2}} d \rho .
$$

Despejando $d u$ y sustituyendo el resultado en (1.31) se obtiene la siguiente expresión para la segunda ley de la termodinámica

$$
d h=T d s+\frac{d p}{\rho} .
$$

la cual es válida para un flujo estacionario o no estacionario. 


\subsubsection{Flujo adiabático ó isoentrópico}

Un flujo en el cual la entropía de cada partícula permanece constante, recibe el nombre de flujo isoentrópico ó adiabático. En este caso la segunda ley (1.32) se reduce a

$$
d h=\frac{d p}{\rho} .
$$

Por otro lado si el flujo es estacionario tenemos

$$
d h=d h(\mathbf{R})=\nabla h \cdot d \mathbf{R} \quad d p=d p(\mathbf{R})=\nabla p \cdot d \mathbf{R}
$$

Sustituyendo estas relaciones en (1.33) se concluye que tiene lugar la relación

$$
\nabla h=\frac{1}{\rho} \nabla p .
$$

A continuación, recordaremos algunas relaciones entre las propiedades de un gas ideal uniforme. La relación entre presión y la energía cinética promedio $\left\langle m v^{2} / 2\right\rangle$ de sus moléculas es

$$
p=\frac{2}{3} \frac{N}{V}\left\langle\frac{m v^{2}}{2}\right\rangle
$$

donde $N$ es el número de partículas en un volumen $V$. Por otro lado tenemos la ecuación de estado de gas ideal

$$
p V=N k T
$$

de donde $k$ es la constante de Boltzmann. Comparando lo anterior obtenemos

$$
k T=\frac{1}{3}\left\langle m v^{2}\right\rangle
$$

lo que puede tomarse como la definición de la escala absoluta de temperatura. Reescribiendo la ecuación de estado

$$
\begin{aligned}
p & =\frac{N m}{V} \frac{k}{m} T \\
& =\frac{M}{V} \frac{k}{m} T
\end{aligned}
$$

aparece la masa $M=N m$ del gas contenido en $V$, la densidad $\rho=M / V$ y la constante

$$
\mathcal{R}=\frac{k}{m}
$$

la cual depende de la masa $m$ de las partículas del gas. Así llegamos a la ecuación de estado

$$
p=\rho \mathcal{R} T \text {. }
$$

Podemos reescribir

$$
\mathcal{R}=\frac{N_{0} k}{N_{0} m}=\frac{\mathcal{R}_{0}}{M}
$$

donde

$$
\mathcal{R}_{0}=N_{0} k=8,314 \mathrm{~J} / \mathrm{mol} K
$$

es la constante universal de los gases. La atmósfera es una mezcla de gases por lo que el valor de $M$ es un promedio de las masas de tales gases y aproximadamente tenemos

$$
\mathcal{R}=287 \mathrm{~J} / \mathrm{kg} K
$$

La primera Ley de la termodinámica

$$
d U=d Q-p d V
$$


da lugar a la definición de la capacidad calorífica a volumen constante

$$
C_{V}=\left(\frac{d Q}{d T}\right)_{V}=\left(\frac{\partial U}{\partial T}\right)_{V} .
$$

Reescribiendo la primera Ley en términos de la entalpía $H=U+p V$ obtenemos

$$
d H=d Q+V d p
$$

con lo cual definimos la capacidad calorífica a presión constante

$$
C_{p}=\left(\frac{d Q}{d T}\right)_{p}=\left(\frac{\partial H}{\partial T}\right)_{p} .
$$

Para obtener la relación entre $C_{p}$ y $C_{V}$ derivamos la identidad

$$
H(T, p)=U(T, V)+p V(T, p)
$$

para obtener

$$
C_{p}=\left(\frac{\partial U}{\partial T}\right)_{p}+p\left(\frac{\partial V}{\partial T}\right)_{p}
$$

Para calcular la derivada sobre $U$ usamos la identidad (proporcionada por la regla de la cadena)

$$
\left(\frac{\partial}{\partial T}\right)_{p}=\left(\frac{\partial}{\partial T}\right)_{V}+\left(\frac{\partial V}{\partial T}\right)_{p}\left(\frac{\partial}{\partial V}\right)_{T},
$$

lo que nos da

$$
C_{p}=C_{V}+\left(\frac{\partial V}{\partial T}\right)_{p}\left(\frac{\partial U}{\partial V}\right)_{T}+p\left(\frac{\partial V}{\partial T}\right)_{p} .
$$

En el caso de un gas ideal tenemos la así llamada Ley de Joule

$$
\left(\frac{\partial U}{\partial V}\right)_{T}=0
$$

la cual junto con la ecuación de estado da

$$
C_{p}=C_{V}+N k
$$

Las relaciones anteriores podemos reescribirlas en términos de cantidades por unidad de masa dividiendo con $M$, cantidades que denotamos con letras minúsculas como arriba, por ejemplo

$$
c_{p}=\frac{C_{p}}{M} \quad c_{V}=\frac{C_{V}}{M}
$$

con lo cual obtenemos

$$
c_{p}=c_{V}+\mathcal{R}
$$

La termodinámica tiene la virtud de proporcionar relaciones entre las variables de estado sin considerar los procesos moleculares involucrados en las transformaciones de un sistema termodinámico. Por ejemplo, consideremos que el gas ideal homogéneo sometido a un proceso adiabático, es decir, no intercambia calor con su entorno. En tal caso la primera Ley se reduce a

$$
d u=\frac{p}{\rho^{2}} d \rho
$$

y si usamos la Ley de Joule (1.35) en la forma

$$
d u=c_{v} d T
$$


obtenemos una ecuación diferencial que sólo involucra variables termodinámicas

$$
c_{v} d T=\frac{p}{\rho^{2}} d \rho
$$

y que podemos integrar con ayuda de la ecuación de estado $p=\rho \mathcal{R} T$ como sigue

$$
c_{v} \frac{d T}{T}=\frac{\mathcal{R}}{\rho} d \rho \rightarrow d \ln T=\frac{\mathcal{R}}{c_{v}} d \ln \rho \rightarrow T=\rho^{\frac{\mathcal{R}}{c_{v}}}+\text { cte } .
$$

por tanto

$$
T=C \rho^{\frac{\mathcal{R}}{c_{v}}}
$$

donde $C$ es una constante de integración.

\subsubsection{Ecuaciones de Poisson}

Hasta aquí hemos considerado un gas ideal con propiedades espacialmente uniformes, pero si invocamos la hipótesis de equilibrio local la última relación es válida para cada partícula independientemente de la forma en que $T$ y $\rho$ dependan de sus coordenadas lagrangianas o eulerianas, es decir, en la deducción anterior hemos trabajado con funciones de la forma $T=T\left(t, x_{0}^{i}\right)$ ó $T\left(t, x^{i}\right)$, de manera que para el caso de un flujo estacionario e isoentrópico podemos escribir

$$
T(\mathbf{r})=C \rho^{\frac{\mathcal{R}}{c_{v}}}(\mathbf{r}) .
$$

Si denotamos con un subíndice 0 al valor de las funciones en $\mathbf{r}_{0}$, tenemos

$$
T_{0}=C \rho_{0}^{\frac{\mathcal{R}}{c_{v}}}
$$

Dividiendo miembro a miembro las dos últimas relaciones se obtiene

$$
T(\mathbf{r})=T_{0}\left(\frac{\rho(\mathbf{r})}{\rho_{0}}\right)^{\frac{\mathcal{R}}{c_{v}}}
$$

o, simplemente,

$$
T=T_{0}\left(\frac{\rho}{\rho_{0}}\right)^{\frac{\mathcal{R}}{c_{v}}}
$$

donde queda implícito que las funciones termodinámicas dependen las coordenadas (lagrangianas o eulerianas) de cada partícula. Usando la ecuación de estado podemos sustituir a $\rho$ por $p$ en (1.38):

$$
\frac{\rho}{\rho_{0}}=\frac{T_{0}}{p_{0}} \frac{p}{T} \rightarrow T=T_{0}\left(\frac{T_{0}}{p_{0}} \frac{p}{T}\right)^{\frac{\mathcal{R}}{c_{v}}} \rightarrow T^{1+\frac{\mathcal{R}}{c_{v}}}=T_{0}^{1+\frac{\mathcal{R}}{c_{v}}}\left(\frac{p}{p_{0}}\right)^{\frac{\mathcal{R}}{c_{v}}}
$$

La relación (1.36) entre capacidades caloríficas equivale a $1+\frac{\mathcal{R}}{c_{v}}=\frac{c_{p}}{c_{v}}$ con lo cual se obtiene

$$
T=T_{0}\left(\frac{p}{p_{0}}\right)^{\frac{\mathcal{R}}{c_{p}}}
$$

Siguiendo el mismo razonamiento sustituyamos $T$ por $\rho$ en (1.38):

$$
\frac{p}{\rho \mathcal{R}}=\frac{p_{0}}{\rho_{0} \mathcal{R}}\left(\frac{p}{p_{0}}\right)^{\frac{\mathcal{R}}{c_{p}}} \rightarrow \rho=\rho_{0} \frac{p}{p_{0}}\left(\frac{p}{p_{0}}\right)^{-\frac{\mathcal{R}}{c_{p}}}=\rho_{0}\left(\frac{p}{p_{0}}\right)^{1-\frac{\mathcal{R}}{c_{p}}}
$$

y usando $1-\frac{\mathcal{R}}{c_{p}}=\frac{c_{v}}{c_{p}}$ se obtiene

$$
\rho=\rho_{0}\left(\frac{p}{p_{0}}\right)^{1 / \gamma}
$$


donde definimos

$$
\gamma \equiv \frac{c_{p}}{c_{v}}
$$

Las ecuaciones (1.38), (1.39), (1.40) reciben el nombre de ecuaciones de Poisson. Para calcular la entalpía integramos (1.37) para obtener

$$
u=c_{v} T
$$

donde, sin perder generalidad, hacemos cero a la constante de integración, entonces

$$
h=u+\frac{p}{\rho}=c_{v} T+\mathcal{R} T
$$

por tanto

$$
h=c_{p} T \text {. }
$$

\subsection{Ecuaciones de Bernoulli en sistemas inerciales}

El propósito de la presente sección es obtener constantes de movimiento a partir de la ecuación de momentum para flujos ideales. Las expresiones de dichas constantes son las Ecuaciones de Bernoulli, ecuaciones de gran interés tanto para la hidrodinámica como para la meteorología. La idea principal para obtener tales ecuaciones es escribir los términos de la ecuación de momentum, para un flujo ideal, como el gradiente de funciones escalares. Recordemos que la ecuación de momentum para un flujo ideal es, en forma Euleriana

$$
\frac{\partial \mathbf{V}}{\partial t}+(\mathbf{V} \cdot \nabla) \mathbf{V}+\frac{1}{\rho} \nabla p-\mathbf{f}(X, t)=0 .
$$

Sustituyendo la siguiente identidad

$$
(\mathbf{V} \cdot \nabla) \mathbf{V}=\nabla \frac{V^{2}}{2}-\mathbf{V} \times(\nabla \times \mathbf{V})
$$

en la ecuación anterior obtenemos

$$
\frac{\partial \mathbf{V}}{\partial t}+\nabla \frac{V^{2}}{2}+\frac{1}{\rho} \nabla p-\mathbf{f}(X, t)=\mathbf{V} \times(\nabla \times \mathbf{V})
$$

Si suponemos que la función $\mathbf{f}$ y el campo $\mathbf{V}$ son el gradiente de alguna función escalar, es decir

$$
\mathbf{f}=-\nabla \Phi_{g} \quad \mathbf{V}=\nabla \Phi_{v}
$$

entonces la ecuación de momentum es

$$
\nabla \frac{\partial \Phi_{v}}{\partial t}+\nabla \frac{V^{2}}{2}+\frac{1}{\rho} \nabla p+\nabla \Phi_{g}=0 .
$$

Ya que, en general, la densidad es función de la posición, debemos utilizar las relaciones termodinámicas desarrolladas en la sección anterior par poder factorizar el operador gradiente.

\subsubsection{Ecuación de Bernoulli para una atmósfera isotérmica}

Si suponemos que la temperatura es constante podemos sustituir la ecuación de estado

$$
\frac{1}{\rho}=\frac{\mathcal{R} T_{0}}{p}
$$

en la ecuación (1.43) para obtener

$$
\nabla \frac{\partial \Phi_{v}}{\partial t}+\nabla \frac{V^{2}}{2}+\frac{\mathcal{R} T_{0} \nabla p}{p}+\nabla \Phi_{g}=0
$$


de donde podemos factorizar el operador gradiente

$$
\nabla\left[\frac{\partial \Phi_{v}}{\partial t}+\frac{V^{2}}{2}+\mathcal{R} T_{0} \ln p+\Phi_{g}\right]=0 .
$$

Lo que quiere decir que

$$
\frac{\partial \Phi_{v}}{\partial t}+\frac{V^{2}}{2}+\mathcal{R} T_{0} \ln p+\Phi_{g}=\mathcal{G}(t)
$$

es una función, a lo más, del tiempo. Si ahora suponemos que el flujo es estacionario entonces

$$
\nabla\left[\frac{V^{2}}{2}+\mathcal{R} T_{0} \ln p+\Phi_{g}\right]=0 .
$$

lo que quiere decir que la función

$$
E(\mathbf{r})=\frac{V^{2}(\mathbf{r})}{2}+\mathcal{R} T_{0} \ln p(\mathbf{r})+\Phi_{g}(\mathbf{r})
$$

tiene el mismo valor numérico en cualquier punto. Para calcular dicho valor suponemos que conocemos el valor de el campo, de la presión y de el potencial en algún punto $\mathbf{r}_{0}$

$$
E\left(\mathbf{r}_{\mathbf{0}}\right)=\frac{V^{2}(\mathbf{r})}{2}+\mathcal{R} T_{0} \ln p\left(\mathbf{r}_{0}\right)+\Phi_{g}\left(\mathbf{r}_{0}\right)=E_{0}
$$

por lo que la Ecuación de Bernoulli para una flujo isotérmico es

$$
\frac{V^{2}(\mathbf{r})}{2}+\mathcal{R} T_{0} \ln p(\mathbf{r})+\Phi_{g}(\mathbf{r})=E_{0}
$$

\subsubsection{Ecuación de Bernoulli para una atmósfera adiabática}

Sustituyendo la ecuación (1.34) en (1.43) podemos factorizar el gradiente:

$$
\nabla\left[\frac{\partial \Phi_{v}}{\partial t}+\frac{V^{2}}{2}+h+\Phi_{g}\right]=0
$$

Nuevamente, para un flujo estacionario tenemos que la constante de movimiento es:

$$
\frac{V^{2}(\mathbf{r})}{2}+h(\mathbf{r})+\Phi_{g}(\mathbf{r})=E_{0}
$$

De (1.39) y (1.41) vemos que

$$
h(\mathbf{r})=c_{p} T_{0}\left(\frac{p(\mathbf{r})}{p_{0}}\right)^{\frac{\nabla}{c_{p}}}
$$

entonces la Ecuación de Bernoulli para una atmósfera adiabática tiene la expresión

$$
\frac{V^{2}(\mathbf{r})}{2}+c_{p} T_{0}\left(\frac{p(\mathbf{r})}{p_{0}}\right)^{\frac{\nabla}{c_{p}}}+\Phi_{g}(\mathbf{r})=E_{0}
$$

Debido al movimiento de rotación de la tierra las ecuaciones desarrolladas hasta el momento no pueden aplicarse directamente. Es necesario extender las ideas presentadas en este capítulo a sistema de referencia no inerciales para expresar las ecuaciones en términos de cantidades relativas a un observador que está parado sobre la superficie terrestre. 


\section{Capítulo 2}

\section{Ecuaciones de movimiento relativas a la tierra}

\subsection{Ecuaciones en un sistema de referencia fijo a la Tierra}

En el capítulo anterior obtuvimos las ecuaciones de balance en un marco inercial $X$ el cual suponemos colocado en el centro de la tierra. Nuestra tarea ahora es transformar dichas ecuaciones a un sistema $Y$ que está fijo a la tierra, es decir, que rota a una velocidad angular constante $\Omega$ alrededor del eje $Y^{3}$.

Para empezar escribimos la base $\left\{\hat{\mathbf{Y}}_{i}\right\}$ en términos de la base $\left\{\hat{\mathbf{X}}_{j}\right\}$.

$$
\begin{aligned}
& \hat{\mathbf{Y}}_{1}=\cos \theta \hat{\mathbf{X}}_{1}+\operatorname{sen} \theta \hat{\mathbf{X}}_{2} \\
& \hat{\mathbf{Y}}_{2}=-\operatorname{sen} \theta \hat{\mathbf{x}}_{1}+\cos \theta \hat{\mathbf{X}}_{2} \\
& \hat{\mathbf{Y}}_{3}=\hat{\mathbf{X}}_{3}
\end{aligned}
$$

o en forma matricial

$$
\left[\begin{array}{c}
\hat{\mathbf{Y}}_{1} \\
\hat{\mathbf{Y}}_{2} \\
\hat{\mathbf{Y}}_{3}
\end{array}\right]=\left[\begin{array}{ccc}
\cos \theta & \operatorname{sen} \theta & 0 \\
-\operatorname{sen} \theta & \cos \theta & 0 \\
0 & 0 & 1
\end{array}\right]\left[\begin{array}{c}
\hat{\mathbf{X}}_{1} \\
\hat{\mathbf{X}}_{2} \\
\hat{\mathbf{X}}_{3}
\end{array}\right] \quad \text { ó } \quad \hat{\mathbf{Y}}_{i}=\mathbb{R}_{i j} \hat{\mathbf{X}}_{j}
$$

La matriz $\mathbb{R}$ tiene las propiedades siguientes

$$
\operatorname{det}(\mathbb{R})=1 \quad \mathbb{R}^{T}=\mathbb{I} .
$$

Ya que el sistema $Y$ está girando el ángulo entre los ejes $X^{1}$ y $Y^{1}$ está dado por $\theta=\Omega t+\theta_{0}$. Por lo que la matriz $\mathbb{R}$ depende del tiempo y, en consecuencia, los vectores base $\left\{\hat{\mathbf{Y}}_{i}\right\}$ también. La expresión del vector de posición, en ambos sistemas es

usando (2.1)

$$
\mathbf{R}=X^{i} \hat{\mathbf{X}}_{i}=Y^{j} \hat{\mathbf{Y}}_{j}
$$

$$
X^{i} \hat{\mathbf{X}}_{i}=Y^{j} \mathbb{R}_{j i} \hat{\mathbf{X}}_{i}
$$

comparando componentes obtenemos la ley de transformación entre componentes de vectores en el sistema $X$ y el sistema $Y$

$$
X^{i}=\mathbb{R}_{i j} Y^{j}, \quad Y^{j}=\mathbb{R}_{j i} X^{i} .
$$

Para calcular la relación entre velocidades no debemos olvidar la dependencia temporal de la base $\left\{\hat{\mathbf{Y}}_{i}\right\}$. Entonces tenemos que el vector velocidad es

$$
\frac{d \mathbf{R}}{d t}=\frac{d X^{i}}{d t} \hat{\mathbf{X}}_{i}=\frac{d}{d t} Y^{j} \hat{\mathbf{Y}}_{j}=\dot{Y}^{j} \hat{\mathbf{Y}}_{j}+Y^{i} \frac{d \hat{\mathbf{Y}}_{j}}{d t}
$$


para calcular $d \hat{\mathbf{Y}}_{j} / d t$ en términos de $\left\{\hat{\mathbf{Y}}_{i}\right\}$ usamos (2.1)

$$
\frac{d \hat{\mathbf{Y}}_{j}}{d t}=\frac{d \mathbb{R}_{j k}}{d t} \hat{\mathbf{X}}_{i}=\frac{d \mathbb{R}_{j k}}{d t} \mathbb{R}_{k j} \hat{\mathbf{Y}}_{j}
$$

Sustituyendo en (2.6)

$$
\frac{d \mathbf{R}}{d t}=\frac{d X^{i}}{d t} \hat{\mathbf{X}}_{i}=\left[\dot{Y}^{j}+Y^{i} \frac{d \mathbb{R}_{j k}}{d t} \mathbb{R}_{k j}\right] \hat{\mathbf{Y}}_{j}
$$

donde

$$
\frac{d \mathbb{R}}{d t} \mathbb{R}^{T}=\left[\begin{array}{ccc}
0 & \Omega & 0 \\
-\Omega & 0 & 0 \\
0 & 0 & 0
\end{array}\right]
$$

Si aplicamos la matriz anterior a un vector cualquiera tenemos

$$
\left[\begin{array}{lll}
A_{1} & A_{2} & A_{3}
\end{array}\right]\left[\begin{array}{ccc}
0 & \Omega & 0 \\
-\Omega & 0 & 0 \\
0 & 0 & 0
\end{array}\right]=\left[\begin{array}{lll}
-\Omega A_{2} & \Omega A_{1} & 0
\end{array}\right] .
$$

Este mismo resultado lo obtenemos del siguiente modo: sea el vector $\boldsymbol{\Omega}=\Omega(0,0,1)^{T}$ entonces

$$
\boldsymbol{\Omega} \times \mathbf{A}=\epsilon_{i j k} \Omega_{j} A_{k}=\left(-\Omega A_{2}, \Omega A_{1}, 0\right) .
$$

Entonces

$$
\mathbf{V}=\dot{X}^{i} \hat{\mathbf{X}}_{i}=\left[\dot{Y}^{j}+\epsilon_{j k l} \Omega_{k} Y^{l}\right] \hat{\mathbf{Y}}_{j}
$$

Para la aceleración tenemos

$$
\begin{aligned}
\mathbf{A} & =\frac{d \mathbf{V}}{d t}=\frac{d}{d t}\left[\dot{Y}^{j}+\epsilon_{j k l} \Omega_{k} Y^{l}\right] \hat{\mathbf{Y}}_{j} \\
& =\left\{\ddot{Y}^{j}+2 \epsilon_{j k l} \Omega_{k} \dot{Y}^{l}+\epsilon_{j k l} \epsilon_{l m n} \Omega_{k} \Omega_{m} Y^{n}\right\} \hat{\mathbf{Y}}_{j} \\
& =\mathbf{a}_{Y}+2 \boldsymbol{\Omega} \times \mathbf{v}+\boldsymbol{\Omega} \times(\boldsymbol{\Omega} \times \mathbf{Y}) .
\end{aligned}
$$

Para el vector gradiente tenemos que

$$
\nabla_{X}=\hat{\mathbf{X}}_{i} \frac{\partial}{\partial X^{i}}
$$

usando regla de la cadena

$$
\nabla_{X}=\hat{\mathbf{X}}_{i} \frac{\partial Y^{k}}{\partial X^{i}} \frac{\partial}{\partial Y^{k}}
$$

de (2.5) vemos que

$$
\frac{\partial Y^{k}}{\partial X^{i}}=\mathbb{R}_{k i}
$$

sustituyendo (2.13) y (2.1) en (2.12)

$$
\nabla_{X}=\mathbb{R}_{i k} \hat{\mathbf{Y}}_{k} \mathbb{R}_{k i} \frac{\partial}{\partial Y^{k}}=\hat{\mathbf{Y}}_{k} \frac{\partial}{\partial Y^{k}}=\nabla_{Y} .
$$

Con lo anterior podemos transformar las ecuaciones de balance. Para obtener la expresión de la densidad en CE $Y$ utilizamos (2.5) para reemplazar la expresión de las CE $X$

$$
\rho(\mathbf{X}, t)=\rho[\mathbf{X}=\mathbf{X}(\mathbf{Y}, t)]=\rho(\mathbf{Y}, t)
$$

por lo que la derivada temporal en ambos sistemas es

$$
\frac{d \rho(\mathbf{X}, t)}{d t}=\frac{d \rho(\mathbf{Y}, t)}{d t} .
$$


Para transformar la velocidad usamos (2.9)

$$
V^{i}=\left[v^{j}+\epsilon_{j k l} \Omega_{k} Y^{l}\right] \mathbb{R}_{j i}
$$

usando regla de la cadena y (2.13) tenemos

$$
\frac{\partial V^{i}}{\partial X^{i}}=\frac{\partial Y^{j}}{\partial X^{i}} \frac{\partial}{\partial Y^{j}}\left[v^{j}+\epsilon_{j k l} \Omega_{k} Y^{l}\right] \mathbb{R}_{j i}=\mathbb{R}_{j i}\left[\frac{\partial v^{j}}{\partial Y^{j}}+\epsilon_{j k l} \Omega_{k} \delta_{j l}\right] \mathbb{R}_{j i}=\mathbb{R}_{j i} \frac{\partial u^{j}}{\partial Y^{j}} \mathbb{R}_{j i}=\frac{\partial u^{j}}{\partial Y^{j}} .
$$

Usando (2.14), (2.16) y (2.17)

$$
\begin{array}{r}
\frac{d \rho(\mathbf{Y}, t)}{d t}+\rho(\mathbf{Y}, t) \frac{\partial v^{j}}{\partial Y^{j}}=0 \\
\frac{d \rho(\mathbf{Y}, t)}{d t}+\rho(\mathbf{Y}, t) \nabla_{Y} \cdot \mathbf{v}(\mathbf{Y}, t)=0 \\
\frac{\partial \rho(\mathbf{Y}, t)}{\partial t}+\nabla_{Y} \cdot \rho(\mathbf{Y}, t) \mathbf{v}(\mathbf{Y}, t)=0 .
\end{array}
$$

En el sistema $X$ la ecuación de momentum es la siguiente

$$
\frac{d \mathbf{V}}{d t}+\frac{1}{\rho} \nabla_{X} p-\mathbf{f}(X, t)=0
$$

Usando (2.10) y (2.14) tenemos

$$
\mathbf{a}_{Y}+2 \boldsymbol{\Omega} \times \mathbf{v}+\boldsymbol{\Omega} \times\{\boldsymbol{\Omega} \times \mathbf{Y}\}+\frac{1}{\rho(\mathbf{Y}, t)} \nabla_{Y} p(\mathbf{Y}, t)-\mathbf{f}(\mathbf{Y}, t)=0
$$

La expresión de la velocidad angular de la tierra en el sistema $Y$ es

$$
\Omega=\Omega \hat{\mathbf{Y}}^{3}
$$

entonces

$$
\begin{aligned}
a_{1}(\mathbf{Y}, t)-2 \Omega Y^{2}-\Omega^{2} Y^{1} & =-\frac{1}{\rho(\mathbf{Y}, t)} \frac{\partial p(\mathbf{Y}, t)}{\partial Y^{1}}+f_{1}(\mathbf{Y}, t) \\
a_{2}(\mathbf{Y}, t)+2 \Omega Y^{1}-\Omega^{2} Y^{2} & =-\frac{1}{\rho(\mathbf{Y}, t)} \frac{\partial p(\mathbf{Y}, t)}{\partial Y^{2}}+f_{2}(\mathbf{Y}, t) \\
a_{3}(\mathbf{Y}, t) & =-\frac{1}{\rho(\mathbf{Y}, t)} \frac{\partial p(\mathbf{Y}, t)}{\partial Y^{3}}+f_{3}(\mathbf{Y}, t)
\end{aligned}
$$

\subsection{Ecuaciones relativas a un plano tangente a la esfera terrestre}

Consideremos un sistema de referencia cartesiano $x^{1} x^{2} x^{3}$ con el plano $x^{1} x^{2}$ tangente a la esfera terrestre en un punto con longitud $\lambda_{c}$ y latitud $\phi_{c}$ como se ilustra en la figura 1.5. La matriz que relaciona a la base asociada al sistema $Y$ con la base asociada al sistema $x^{1} x^{2} x^{3}$ es

$$
\mathbb{R}_{c}=\left[\begin{array}{ccc}
-\operatorname{sen} \lambda_{c} & \cos \lambda_{c} & 0 \\
-\cos \lambda_{c} \operatorname{sen} \phi_{c} & -\operatorname{sen} \lambda_{c} \operatorname{sen} \phi_{c} & \cos \phi_{c} \\
\cos \lambda_{c} \cos \phi_{c} & \operatorname{sen} \lambda_{c} \cos \phi_{c} & \operatorname{sen} \phi_{c}
\end{array}\right]
$$

donde el punto $\xi=\left(\lambda_{f}, \phi_{f}, r_{f}\right)$. La relación de vectores base esta dada por

$$
\hat{\mathbf{x}}_{i}=\mathbb{R}_{c i j}(\xi) \hat{\mathbf{Y}}_{j} .
$$

La relación que tienen las componentes de cualquier vector en ambos sistema es la siguiente

$$
A_{i}(\mathbf{Y}) \hat{\mathbf{Y}}_{i}=A_{j}(\mathbf{x}) \hat{\mathbf{x}}_{j}
$$

utilizando $(2.21)$

$$
A_{i}(\mathbf{Y}) \hat{\mathbf{Y}}_{i}=A_{j}(\hat{\mathbf{x}}) \mathbb{R}_{c j i} \hat{\mathbf{Y}}_{i}
$$



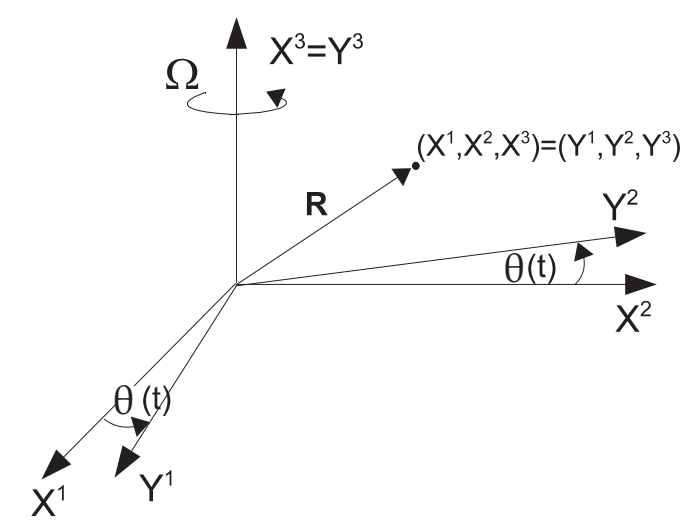

Figura 2.1: Sistemas de referencia inercial $X^{i}$ y primario $Y^{j}$.

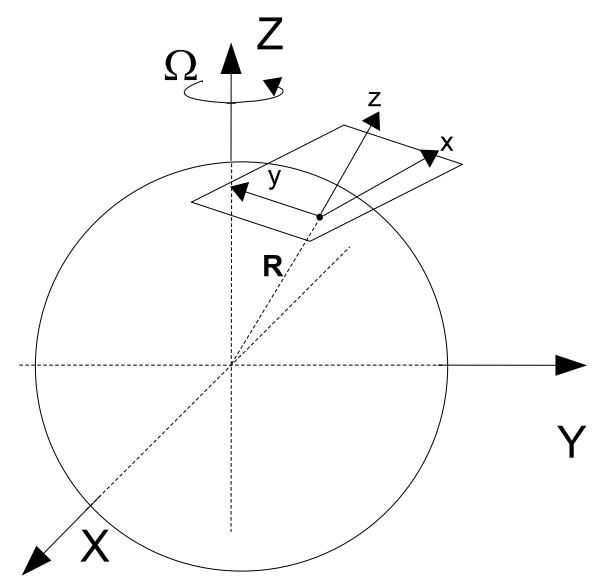

Figura 2.2: Sistema de referencia primario $Y^{i}$ y coordenadas relativas a un plano tangente. 
comparando componentes obtenemos la relación buscada

$$
A_{i}(\mathbf{Y})=A_{j}(\hat{\mathbf{x}}) \mathbb{R}_{c j i}
$$

El vector de posición está dado por

$$
\mathbf{R}=Y^{i} \hat{\mathbf{Y}}_{i}=\left(x^{j}+\delta_{3 j} a\right) \hat{\mathbf{x}}_{j}
$$

donde $a$ es el radio terrestre. Para la transformación del vector velocidad utilizaremos la expresión

$$
\mathbf{V}=\mathbf{v}+\boldsymbol{\Omega} \times \mathbf{R}
$$

Donde

$$
\mathbf{v}=\frac{d Y^{i}}{d t} \hat{\mathbf{Y}}_{i}
$$

usando regla de la cadena, (2.21) y (2.22)

$$
\mathbf{v}=\dot{x}^{k} \frac{\partial x^{j} \mathbb{R}_{i j}}{\partial x^{k}} \mathbb{R}_{j i} \hat{\mathbf{x}}_{j}=\dot{x}^{k} \frac{\partial x^{j}}{\partial x^{k}} \mathbb{R}_{i j} \mathbb{R}_{j i} \hat{\mathbf{x}}_{j}=\dot{x}^{k} \delta_{k j} \hat{\mathbf{x}}_{j}=\dot{x}^{k} \hat{\mathbf{x}}_{k}=w^{k} \hat{\mathbf{x}}_{k}
$$

La velocidad angular tiene la siguiente expresión en el sistema $X_{P}$

$$
\boldsymbol{\Omega}=\Omega\left\{\hat{\mathbf{x}}_{2} \cos \phi+\hat{\mathbf{x}}_{3} \operatorname{sen} \phi\right\}
$$

entonces

$\boldsymbol{\Omega} \times \mathbf{Y}=\Omega\left\{\hat{\mathbf{x}}_{2} \cos \phi+\hat{\mathbf{x}}_{3} \operatorname{sen} \phi\right\} \times\left(x^{j}+\delta_{3 j} a\right) \hat{\mathbf{x}}_{j}=\Omega\left\{\left[\left(x^{3}+a\right) \cos \phi-x^{2} \operatorname{sen} \phi\right] \hat{\mathbf{x}}_{1}+x^{1} \operatorname{sen} \phi \hat{\mathbf{x}}_{2}-x^{1} \cos \phi \hat{\mathbf{x}}_{3}\right\}$

Usando (2.23) y (2.25) obtenemos la velocidad en términos de cantidades medidas en el sistema $x^{1}, x^{2}, x^{3}$

$$
\left[\begin{array}{l}
V^{1} \\
V^{2} \\
V^{3}
\end{array}\right]=\left[\begin{array}{c}
w^{1}+\Omega\left[\left(x^{3}+a\right) \cos \phi-x^{2} \operatorname{sen} \phi\right] \\
w^{2}+x^{1} \Omega \operatorname{sen} \phi \\
w^{3}-x^{1} \Omega \cos \phi
\end{array}\right]
$$

Para la aceleración se hace algo similar, de la expresión

$$
\mathbf{A}=\mathbf{a}+2 \boldsymbol{\Omega} \times \mathbf{v}+\boldsymbol{\Omega} \times \boldsymbol{\Omega} \times \mathbf{R}
$$

se transforma la aceleración en el sistema $Y$ utilizando (2.21) y (2.22)

$$
\mathbf{a}=\frac{d v^{i}}{d t} \hat{\mathbf{Y}}_{i}=\frac{d w^{j}}{d t} \mathbb{R}_{i j} \mathbb{R}_{j i} \hat{\mathbf{x}}_{j}=\frac{d w^{j}}{d t} \hat{\mathbf{x}}_{j}
$$

Ahora se transforma $2 \boldsymbol{\Omega} \times \mathbf{v}$ utilizando (2.23) y (2.24)

$$
\begin{aligned}
\boldsymbol{\Omega} \times \mathbf{v} & =\Omega\left\{\hat{\mathbf{x}}_{2} \cos \phi+\hat{\mathbf{x}}_{3} \operatorname{sen} \phi\right\} \times w^{k} \hat{\mathbf{x}}_{k} \\
& =\Omega\left\{\left(w^{3} \cos \phi-w^{2} \operatorname{sen} \phi\right) \hat{\mathbf{x}}_{1}+w^{1} \operatorname{sen} \phi \hat{\mathbf{x}}_{2}-w^{1} \cos \phi \hat{\mathbf{x}}_{3}\right\} .
\end{aligned}
$$

Para la aceleración centrípeta tenemos

$$
\begin{aligned}
\boldsymbol{\Omega} \times \boldsymbol{\Omega} \times \mathbf{R} & =\Omega\left\{\hat{\mathbf{x}}_{2} \cos \phi+\hat{\mathbf{x}}_{3} \operatorname{sen} \phi\right\} \times \boldsymbol{\Omega} \times \mathbf{R} \\
& =\Omega^{2}\left\{-x^{1} \hat{\mathbf{x}}_{1}+\operatorname{sen} \phi\left[\left(x^{3}+a\right) \cos \phi-x^{2} \operatorname{sen} \phi\right] \hat{\mathbf{x}}_{2}-\cos \phi\left[\left(x^{3}+a\right) \cos \phi-x^{2} \operatorname{sen} \phi\right] \hat{\mathbf{x}}_{3}\right\} .
\end{aligned}
$$

Finalmente tenemos

$$
\mathbf{A}=\left[\begin{array}{c}
\dot{w}^{1}+2 \Omega\left(w^{3} \cos \phi-w^{2} \operatorname{sen} \phi\right)-\Omega^{2} x^{1} \\
\dot{w}^{2}+2 w^{1} \Omega \operatorname{sen} \phi+\Omega^{2} \operatorname{sen} \phi\left[\left(x^{3}+a\right) \cos \phi-x^{2} \operatorname{sen} \phi\right] \\
\dot{w}^{3}-2 w^{1} \Omega \cos \phi-\Omega^{2} \cos \phi\left[\left(x_{3}+a\right) \cos \phi-x^{2} \operatorname{sen} \phi\right]
\end{array}\right]
$$


La transformación de gradiente es como sigue

$$
\nabla=\hat{\mathbf{Y}}_{i} \frac{\partial}{\partial Y^{i}}=\mathbb{R}_{j i} \hat{\mathbf{x}}_{j} \frac{\partial x^{j}}{\partial Y^{i}} \frac{\partial}{\partial x^{j}}=\mathbb{R}_{j i} \hat{\mathbf{x}}_{j} \mathbb{R}_{j i} \frac{\partial}{\partial x^{j}}=\hat{\mathbf{x}}_{j} \mathbb{R}_{i j} \mathbb{R}_{j i} \frac{\partial}{\partial x^{j}}=\hat{\mathbf{x}}_{j} \frac{\partial}{\partial x^{j}}
$$

Para transformar la ecuación de continuidad

$$
\frac{d \rho(\mathbf{R}, t)}{d t}+\rho(\mathbf{R}, t) \frac{\partial v^{i}}{\partial Y^{i}}=0
$$

utilizamos (2.22) y la regla de la cadena.

$$
\frac{\partial v^{i}}{\partial Y^{i}}=\frac{\partial w^{j}}{\partial Y^{i}} \mathbb{R}_{i j}=\frac{\partial x^{k}}{\partial Y^{i}} \frac{\partial w^{j}}{\partial x^{k}} \mathbb{R}_{i j}=\frac{\partial w^{j}}{\partial x^{k}} \mathbb{R}_{i j} \mathbb{R}_{j i}=\frac{\partial w^{j}}{\partial x^{k}} \delta_{j k}=\frac{\partial w^{j}}{\partial x^{j}}
$$

Entonces la ecuación de continuidad en el sistema $x^{1}, x^{2}, x^{3}$ queda

$$
\frac{d \rho(\mathbf{x}, t)}{d t}+\rho(\mathbf{x}, t) \frac{\partial w^{i}}{\partial x^{i}}=0 .
$$

Para transformar la ecuación de momentum

$$
\mathbf{a}+2 \boldsymbol{\Omega} \times \mathbf{v}+\boldsymbol{\Omega} \times(\boldsymbol{\Omega} \times \mathbf{R})=-\frac{1}{\rho(\mathbf{R}, t)} \nabla_{Y} p+\mathbf{g}+\mathbf{f}(\mathbf{R}, t)
$$

utilizamos $(2.27),(2.28)$ y la regla de la cadena. Por lo que tenemos

$$
\begin{aligned}
\dot{w}^{1}+2 \Omega\left(w^{3} \cos \phi-w^{2} \operatorname{sen} \phi\right)-\Omega^{2} x^{1} & =-\frac{1}{\rho(\mathbf{x}, t)} \frac{\partial p}{\partial x^{1}}+g_{1}+f_{1}(\mathbf{x}, t) \\
\dot{w}^{2}+2 w^{1} \Omega \operatorname{sen} \phi+\Omega^{2} \operatorname{sen} \phi\left[\left(x^{3}+a\right) \cos \phi-x^{2} \operatorname{sen} \phi\right] & =-\frac{1}{\rho(\mathbf{x}, t)} \frac{\partial p}{\partial x^{2}}+g_{2}+f_{2}(\mathbf{x}, t) \\
\dot{w}^{3}-2 w^{1} \Omega \cos \phi-\Omega^{2} \cos \phi\left[\left(x^{3}+a\right) \cos \phi-x^{2} \operatorname{sen} \phi\right] & =-\frac{1}{\rho(\mathbf{x}, t)} \frac{\partial p}{\partial x^{3}}+g_{3}+f_{2}(\mathbf{x}, t)
\end{aligned} .
$$

Que es la ecuación de momentum relativa a un plano tangente a la esfera en el punto $\xi=\left(\lambda_{f}, \phi_{f}, r_{f}\right)$. Para simplificar las ecuaciones anteriores despreciamos los términos que contengan $\Omega^{2}$ pues se consideran pequeños con respecto a los demás. Además consideramos que la única fuerza volumétrica de importancia es la gravedad, que en este caso tiene la expresión

$$
\mathbf{g}=-g \frac{a^{2}}{r^{3}}\left(x_{i}+\delta_{i 3} a\right) \hat{\mathbf{x}}_{i} \quad g=\frac{G M_{T}}{a^{2}}
$$

donde $G$ es la contante gravitacional, $M_{T}$ es la masa terrestre, $a$ es el radio terrestre y

$$
r=\left[\left(x^{1}\right)^{2}+\left(x^{2}\right)^{2}+\left(x^{3}+a\right)^{2}\right]^{3 / 2}
$$

Con esto obtenemos la ecuación de momentum

$$
\begin{aligned}
\dot{w}^{1}+2 \Omega\left(w^{3} \cos \phi-w^{2} \operatorname{sen} \phi\right) & =-\frac{1}{\rho(\mathbf{x}, t)} \frac{\partial p}{\partial x^{1}}-g a^{2} r^{-3} x^{1} \\
\dot{w}^{2}+2 w^{1} \Omega \operatorname{sen} \phi & =-\frac{1}{\rho(\mathbf{x}, t)} \frac{\partial p}{\partial x^{2}}-g a^{2} r^{-3} x^{2} \\
\dot{w}^{3}-2 w^{1} \Omega \cos \phi & =-\frac{1}{\rho(\mathbf{x}, t)} \frac{\partial p}{\partial x^{2}}-g a^{2} r^{-3}\left(x^{3}+a\right) .
\end{aligned}
$$

\subsection{Ecuaciones de momentum aproximadas y su región de validez}

El problema de la validez local de las ecuaciones de momentum fué señalado por McVitte en 1948 [21] y replanteado por Nuñez $[27,29,28]$ en años recientes.

Es necesario hacer notar que en la literatura convencional de meteorología, se utiliza la aproximación para la aceleración de la gravedad

$$
\mathbf{g}=-g \hat{\mathbf{x}}_{3}
$$


con esta aproximación las ecuaciones de momentum quedan

$$
\begin{aligned}
\dot{w}^{1}+2 \Omega\left(w^{3} \cos \phi-w^{2} \operatorname{sen} \phi\right) & =-\frac{1}{\rho(\mathbf{x}, t)} \frac{\partial p}{\partial x^{1}} \\
\dot{w}^{2}+2 w^{1} \Omega \operatorname{sen} \phi & =-\frac{1}{\rho(\mathbf{x}, t)} \frac{\partial p}{\partial x^{2}} \\
\dot{w}^{3}-2 w^{1} \Omega \cos \phi & =-\frac{1}{\rho(\mathbf{x}, t)} \frac{\partial p}{\partial x^{3}}-g .
\end{aligned}
$$

Para mostrar que los términos que se han omitido en (2.32) son los dominantes, a partir de 100 km, mostramos la tabla (2.1) con los órdenes de magnitud de los términos de (2.31).

\begin{tabular}{|c|c|c|c|c|c|}
\hline & $\frac{d u}{d t}$ & $-\frac{1}{\rho} \frac{\partial p}{\partial x^{1}}$ & $+f v$ & $-f w$ & $-\frac{g a^{2} x^{1}}{r^{3}}$ \\
\hline$L$ & $\frac{U^{2}}{L}$ & $\frac{\Delta p}{\rho L}$ & $f U$ & $\frac{f H U}{L}$ & \\
$10^{6}$ & $10^{-4}$ & $10^{-3}$ & $10^{-2}$ & $10^{-5}$ & $10^{0}$ \\
$10^{5}$ & $10^{-3}$ & $10^{-2}$ & $10^{-3}$ & $10^{-4}$ & $10^{-1}$ \\
$10^{4}$ & $10^{-2}$ & $10^{-1}$ & $10^{-3}$ & $10^{-3}$ & $10^{-2}$ \\
\hline
\end{tabular}

Cuadro 2.1: Magnitudes en $\mathrm{ms}^{-2}$ de los términos de las ecuaciones de momentum. Donde $L(\mathrm{~m})$ es la escala horizontal, $U=10 \mathrm{~ms}^{-1}, H=10^{4} \mathrm{~m}, f=2 \Omega \operatorname{sen} \phi, \phi=45^{\circ}, g=10 \mathrm{~ms}^{-2}, x_{1}=L / 2, x_{2}=x_{3}=0$, $r=\sqrt{x_{1}^{2}+a^{2}}, a=6378 \mathrm{~km}$

Para estimar la región de validez se utiliza (2.31) y (2.32) con el fin de obtener la presión de una atmósfera isotérmica en reposo con respecto a la tierra, con la condición $p\left(z_{i}=0\right)=p_{0}$. Esto es, resolvemos las ecuaciones

$$
\begin{aligned}
& \frac{1}{p} \frac{d p}{d x^{i}}=-\frac{g a^{2}}{\mathcal{R} T} \frac{x^{i}}{r^{3}} \\
& \frac{1}{p} \frac{d p}{d x^{3}}=-\frac{g}{\mathcal{R} T} .
\end{aligned}
$$

La expresión de la densidad la obtenemos de la ley de gas ideal $\rho=p / \mathcal{R} T$. Las soluciones a las ecuaciones (2.33a) y (2.33b) son, respectivamente

$$
\begin{aligned}
& p_{E}=p_{0} e^{-b a\left(1-\frac{a}{r}\right)} \\
& p_{A}=p_{0} e^{-b x^{3}}
\end{aligned}
$$

con $b=g / \mathcal{R} T$. Donde (2.34) es la presión en cualquier parte de la esfera terrestre con superficies esféricas de presión constante y (2.35) es la presión aproximada, la cual es constante en el plano $x^{3}=$ cte. Con estas dos últimas expresiones calculamos la presión sobre la esfera terrestre

$$
p_{E}=p_{0} \quad p_{A}=p_{0} e^{b\left(a-\sqrt{a^{2}-\xi^{2}}\right)}
$$

donde

$$
x^{3}=-a+\sqrt{a^{2}-\xi^{2}} \quad \xi^{2}=\left(x^{1}\right)^{2}+\left(x^{3}\right)^{2} .
$$

Entonces, el error relativo es

$$
\begin{aligned}
& \Delta p=\left|\frac{p_{A}}{p_{E}}-1\right| \times 100 \\
& \Delta p=100\left[e^{b\left(a-\sqrt{a^{2}-\xi^{2}}\right)}-1\right] .
\end{aligned}
$$

Si usamos los valores $g=9,8 \mathrm{~ms}^{-2}, T=300 \mathrm{~K}, \mathcal{R}=287 \mathrm{~J} / \mathrm{kgK}, a=6378 \mathrm{~km}$ se obtiene $b=0,11382 \mathrm{~km}^{-1}$. En la figura (2.3) se observa la dependencia de el error relativo con la distancia del origen a un punto sobre el plano $x^{3}=0$. De aquí se puede ver que, si el error máximo permitido es de $5 \%$, entonces la región máxima es de un radio de $75 \mathrm{~km}$ por lo que el dominio horizontal máximo es de $150 \mathrm{~km} \times 150 \mathrm{~km}$. 


\subsection{Ecuaciones en coordenadas curvilíneas ortogonales $s^{i}$ defini- das en el sistema primario $Y^{i}$}

Para obtener las ecuaciones de mometum en coorenadas curvileneas seguimos el formalismo de matrices de rotacion usado en $[27,28]$

Consideremos que las coordenadas $Y^{i}$ dependen de otras coordenadas $s^{1} s^{2} s^{3}$

$$
Y^{i}=Y^{i}\left(s^{1}, s^{2}, s^{3}\right) \equiv Y^{i}(s)
$$

donde no aparece explícitamente el tiempo $t$ (ver figura 2.4) de manera que

$$
\mathbf{R}=\mathbf{R}(s, t)=Y^{i}(s) \widehat{\mathbf{Y}}_{i}(t) .
$$

La base covariante $\tau_{i}$ se define por

$$
\tau_{i}=\left(\frac{\partial \mathbf{R}}{\partial s^{i}}\right)_{t}=\frac{\partial Y^{j}}{\partial s^{i}} \widehat{\mathbf{Y}}_{j}
$$

y los factores métricos $h_{i}$ por

$$
h_{i} \equiv\left\|\tau_{i}\right\|
$$

Consideremos que el sistema curvilíneo $s^{i}$ es ortogonal, esto es, los vectores unitarios

$$
\widehat{\mathbf{s}}_{i}=\frac{\tau_{i}}{h_{i}} \quad(\text { sin sumar en i) }
$$

forman una base ortonormal,

$$
\widehat{\mathbf{s}}_{i} \cdot \widehat{\mathbf{s}}_{j}=\delta_{i j}
$$

Además supondremos que el orden de $s^{1}, s^{2}, s^{3}$ es tal que los vectores $\widehat{\mathbf{s}}^{i}$ obedecen la regla de la mano derecha,

$$
\widehat{\mathbf{s}}_{i} \times \widehat{\mathbf{s}}_{j}=\epsilon_{i j k} \widehat{\mathbf{s}}_{k}
$$

de manera que la base $\widehat{\mathbf{s}}_{i}$ es una rotación rígida de $\widehat{\mathbf{Y}}_{1}, \widehat{\mathbf{Y}}_{2}, \widehat{\mathbf{Y}}_{3}$. Esto implica que existe una matriz de rotación $\mathbb{R}$ tal que

$$
\widehat{\mathbf{s}}^{i}=\mathbb{R}_{i j} \widehat{\mathbf{Y}}^{j}
$$

satisface $\operatorname{det}(\mathbb{R})=1 \mathrm{y}$

$$
\mathbb{R}^{T}=\mathbb{I} \quad \text { ó } \quad \mathbb{R}_{i j} \mathbb{R}_{k j}=\delta_{i k}
$$

de donde se obtiene

$$
\widehat{\mathbf{Y}}^{j}=\mathbb{R}_{i j} \widehat{\mathbf{s}}^{i}
$$

La relación

$$
\tau_{i}=h_{i} \widehat{\mathbf{s}}_{i}=\frac{\partial Y^{j}}{\partial s^{i}} \widehat{\mathbf{Y}}_{j}
$$

nos da

$$
\widehat{\mathbf{s}}_{i}=\frac{1}{h_{i}} \frac{\partial Y^{j}}{\partial s^{i}} \widehat{\mathbf{Y}}_{j} \quad(\text { sin sumar en } i) .
$$

Comparando con (2.42) obtenemos las componentes de $\mathbb{R}$ :

$$
\mathbb{R}_{i j}=\frac{1}{h_{i}} \frac{\partial Y^{j}}{\partial s^{i}} \quad(\text { sin sumar en } i) .
$$

La matriz Jacobiana $\mathbb{J}$ de la transformación (2.37) es

$$
\mathbb{J}_{i j}=\frac{\partial Y^{j}}{\partial s^{i}}
$$




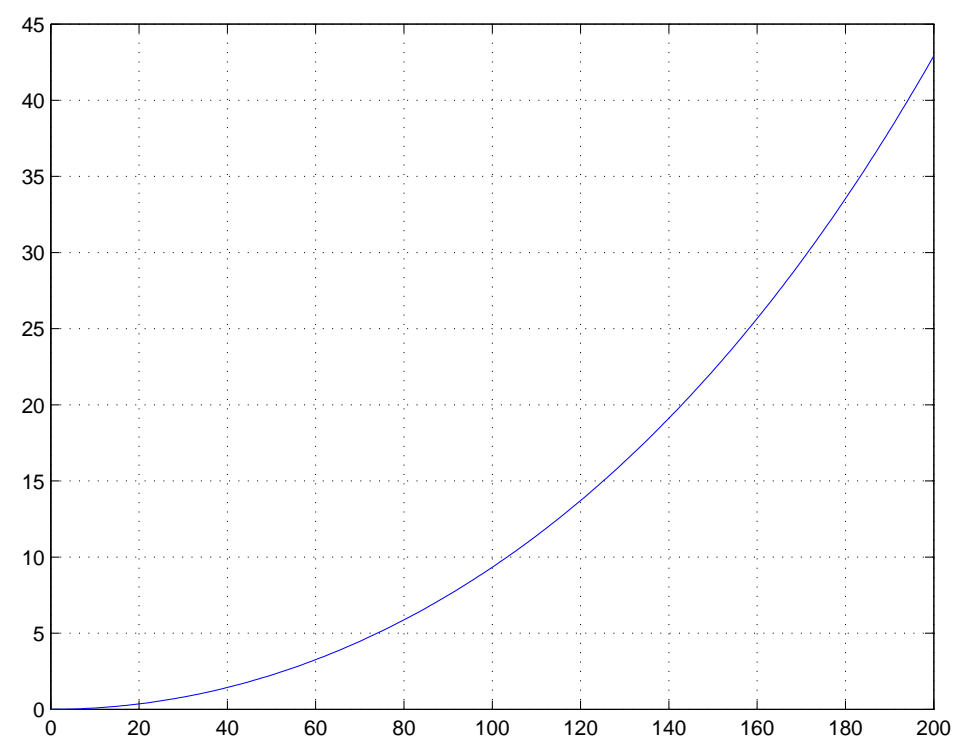

Figura 2.3: Error relativo vs región de validez

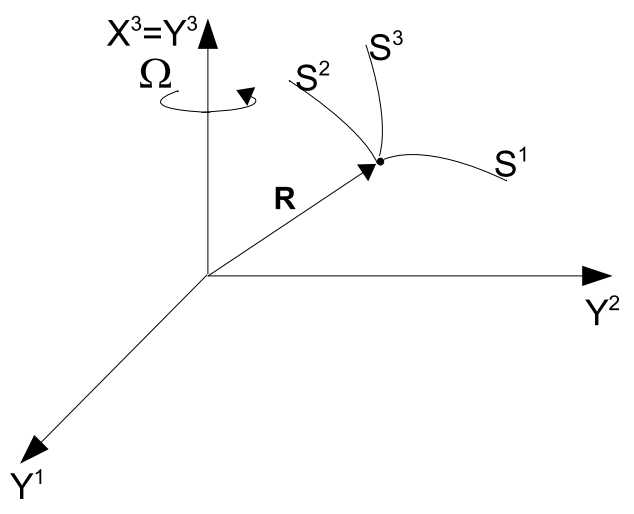

Figura 2.4: Sistema curvilíneo $s^{1} s^{2} s^{3}$. 
y el Jacobiano correspondiente es

$$
\begin{aligned}
J & =\operatorname{det}\left(\mathbb{J}_{i j}\right)=\tau_{1} \cdot\left(\tau_{2} \times \tau_{3}\right) \\
& =h_{1} \widehat{\mathbf{s}}_{1} \cdot\left(h_{2} \widehat{\mathbf{s}}_{2} \times h_{3} \widehat{\mathbf{s}}_{3}\right) \\
& =h_{1} h_{2} h_{3} .
\end{aligned}
$$

Ahora consideremos la transformación de operaciones vectoriales para obtener las ecuaciones de movimiento en las coordenadas $s^{i}$ :

a) Para la transformación de las componentes de un vector consideremos

$$
\mathbf{b}=b^{j}(Y) \widehat{\mathbf{Y}}_{j}=b^{i}(s) \widehat{\mathbf{s}}_{i} .
$$

Sustituyendo (2.44) obtenemos

$$
b^{i}(s)=\mathbb{R}_{i j} b^{j}(Y),
$$

y, usando (2.43),

$$
b_{j}(Y)=\mathbb{R}_{i j} b^{i}(s) .
$$

En particular, para el vector de posición

$$
\mathbf{R}=[\mathbf{R}]_{s}^{i} \widehat{\mathbf{s}}_{i}=Y^{j}(s) \widehat{\mathbf{Y}}_{j}(t)
$$

tenemos

$$
[\mathbf{R}]_{s}^{i}=\mathbb{R}_{i j} Y^{j}(s) .
$$

Para el producto vectorial

$$
\mathbf{a} \times \mathbf{b}=\mathbf{c}
$$

consideremos

$$
\mathbf{a}=a^{i} \widehat{\mathbf{s}}_{i} \quad \mathbf{b}=b^{j} \widehat{\mathbf{s}}_{j} \quad \mathbf{c}=c^{l} \widehat{\mathbf{s}}_{l},
$$

entonces

$$
a^{i} b^{j} \widehat{\mathbf{s}}_{i} \times \widehat{\mathbf{s}}_{j}=c^{l} \widehat{\mathbf{s}}_{l},
$$

multiplicando por $\widehat{\mathbf{s}}_{k} \cdot$ y usando (2.41) se obtiene

$$
c^{k}=\epsilon_{i j k} a^{i} b^{j}
$$

lo que significa que podemos usar la formula del determinante para calcular $\mathbf{a} \times \mathbf{b}$,

$$
\mathbf{a} \times \mathbf{b}=\left|\begin{array}{ccc}
\widehat{\mathbf{s}}_{1} & \widehat{\mathbf{s}}_{2} & \widehat{\mathbf{s}}_{3} \\
a_{1} & a_{2} & a_{3} \\
b_{1} & b_{2} & b_{3}
\end{array}\right|
$$

b) Para obtener la expresión de la velocidad

$$
\mathbf{V}=\frac{d \mathbf{R}}{d t}=\mathbf{v}+\mathbf{\Omega} \times \mathbf{R}
$$

en el sistema $s^{j}$ comenzamos por la transformación de la velocidad $\mathbf{v}$ relativa a la Tierra:

$$
\mathbf{v}=\dot{Y^{i}} \widehat{\mathbf{Y}}^{i}=\widehat{\mathbf{Y}}_{i} \frac{d}{d t} Y^{i}(s)=\widehat{\mathbf{Y}}_{i} \frac{\partial Y^{i}}{\partial s^{j}} \dot{s}^{j} \quad \text { (usando regla de la cadena) }
$$

donde aparece la definición (2.39), por tanto

$$
\mathbf{v}=\tau_{j} \dot{s}^{j}
$$

Sustituyendo (2.45) obtenemos

$$
\mathbf{v}=\widehat{\mathbf{s}}_{j} v^{j} \quad \text { con } \quad v^{j} \equiv h_{j} \dot{s}^{j} \quad(\text { sin sumar en } j)
$$


siendo $v^{j}$ las componentes físicas de $\mathbf{v}$ en la base $\widehat{\mathbf{s}}_{j} \mathrm{y}$

$$
\dot{s}^{j}=\frac{v^{j}}{h_{j}}(\text { sin sumar en } j)
$$

son las velocidades generalizadas. Para el término $\Omega \times \mathbf{R}$ usamos (2.52)

$$
\boldsymbol{\Omega} \times \mathbf{R}=\Omega^{i} \widehat{\mathbf{s}}_{i} \times[\mathbf{R}]_{s}^{j} \widehat{\mathbf{s}}_{j}=\left|\begin{array}{ccc}
\widehat{\mathbf{s}}_{1} & \widehat{\mathbf{s}}_{2} & \widehat{\mathbf{s}}_{3} \\
\Omega^{1} & \Omega^{2} & \Omega^{3} \\
{[\mathbf{R}]_{s}^{1}} & {[\mathbf{R}]_{s}^{2}} & {[\mathbf{R}]_{s}^{3}}
\end{array}\right|
$$

donde las componentes $\Omega^{i}$ están dadas por

$$
\left(\begin{array}{l}
\Omega_{1} \\
\Omega_{2} \\
\Omega_{3}
\end{array}\right)_{s}=\mathbb{R}\left(\begin{array}{l}
0 \\
0 \\
\Omega
\end{array}\right)_{Y} .
$$

c) Para la aceleración tenemos

$$
\mathbf{A}=\frac{d \mathbf{V}}{d t}=\mathbf{a}+2 \boldsymbol{\Omega} \times \mathbf{v}+\boldsymbol{\Omega} \times(\boldsymbol{\Omega} \times \mathbf{R}) .
$$

(i) Para transformar la aceleración relativa a la Tierra

$$
\mathbf{a}=\dot{v}^{j}(Y) \widehat{\mathbf{Y}}_{j}
$$

usamos la relación (2.50) entre componentes físicas de $\mathbf{v}$ en la bases $\widehat{\mathbf{Y}}_{j}$ y $\widehat{\mathbf{s}}_{i}$, a saber,

$$
v^{j}(Y)=\mathbb{R}_{i j} v^{i}(s) .
$$

Derivando

$$
\dot{v}^{j}(Y)=\mathbb{R}_{i j} \dot{v}^{i}(s)+\dot{\mathbb{R}}_{i j} v^{i}(s)
$$

y usando (2.44) se obtiene

$$
\mathbf{a}=\left(\mathbb{R}_{i j} \dot{v}^{i}+\dot{\mathbb{R}}_{i j} v^{i}\right) \mathbb{R}_{k j} \widehat{\mathbf{s}}_{k}=\left(\mathbb{R}_{i j} \mathbb{R}_{k j} \dot{v}^{i}+\mathbb{R}_{k j} \dot{\mathbb{R}}_{i j} v^{i}\right) \widehat{\mathbf{s}}_{k}=\left(\dot{v}^{k}+\mathbb{R}_{k j} \dot{\mathbb{R}}_{i j} v^{i}\right) \widehat{\mathbf{s}}_{k}
$$

donde identificamos a las componentes físicas $a^{k}$ de $\mathbf{a}$,

$$
\mathbf{a}=a^{k} \widehat{\mathbf{s}}_{k},
$$

a saber,

$$
a^{k}=\dot{v}^{k}+\mathbb{R}_{k j} \dot{\mathbb{R}}_{i j} v^{i}
$$

expresión que tiene la forma matricial

$$
\left(\begin{array}{c}
a^{1} \\
a^{2} \\
a^{3}
\end{array}\right)=\left(\begin{array}{c}
\dot{v}^{1} \\
\dot{v}^{2} \\
\dot{v}^{3}
\end{array}\right)+\mathbb{R}^{T}\left(\begin{array}{c}
v^{1} \\
v^{2} \\
v^{3}
\end{array}\right) .
$$

(ii) El cálculo de la matriz antisimétrica $\mathbb{R}^{T}$ se hace con la regla de la cadena

$$
\frac{d}{d t} \mathbb{R}(s)=\dot{s}^{n} \frac{\partial \mathbb{R}}{\partial s^{n}}
$$

donde $\dot{s}^{n}$ se expresa en términos de las componentes físicas de $\mathbf{v}$ [ec. (2.55)], en esta forma

$$
\mathbb{R} \dot{\mathbb{R}}^{T}=\frac{v^{n}}{h_{n}} \mathbb{R} \frac{\partial \mathbb{R}^{T}}{\partial s^{n}}
$$


donde sólo hay que calcular explícitamente las matrices antisimétricas

$$
\mathbb{R} \frac{\partial \mathbb{R}^{T}}{\partial s^{n}} .
$$

(iii) El término $\boldsymbol{\Omega} \times \mathbf{v}$ se calcula en la forma usual

$$
\boldsymbol{\Omega} \times \mathbf{v}=\boldsymbol{\Omega}^{i} \widehat{\mathbf{s}}_{i} \times v^{j} \widehat{\mathbf{s}}_{j}=\left|\begin{array}{ccc}
\widehat{\mathbf{s}}_{1} & \widehat{\mathbf{s}}_{2} & \widehat{\mathbf{s}}_{3} \\
\Omega^{1} & \Omega^{2} & \Omega^{3} \\
v^{1} & v^{2} & v^{3}
\end{array}\right| .
$$

mientras que $\boldsymbol{\Omega} \times(\boldsymbol{\Omega} \times \mathbf{R})$ puede calcularse en forma análoga.

d) Para transformar el operador $\nabla$ introducimos la base contravariante

$$
\eta^{i} \equiv \nabla s_{i}=\widehat{\mathbf{Y}}^{j} \frac{\partial s^{i}}{\partial Y^{j}} .
$$

El par de bases $\tau_{k}, \eta^{i}$ es recíproco, es decir, satisface

$$
\eta^{i} \cdot \tau_{k}=\delta_{k}^{i} .
$$

En efecto, tenemos

$$
\eta^{i} \cdot \tau_{k}=\widehat{\mathbf{Y}}^{j} \frac{\partial s^{i}}{\partial Y^{j}} \cdot \widehat{\mathbf{Y}}_{l} \frac{\partial Y^{l}}{\partial s^{k}}=\delta_{l}^{j} \frac{\partial s^{i}}{\partial Y^{j}} \frac{\partial Y^{l}}{\partial s^{k}}=\frac{\partial s^{i}}{\partial s^{k}}=\delta_{k}^{i} .
$$

Usando la reciprocidad es fácil obtener

$$
\begin{array}{ll}
\eta^{i} & =\frac{1}{h_{i}} \widehat{\mathbf{s}}_{i} \quad(\text { sin sumar en } i) \\
\widehat{\mathbf{s}}_{i} & =h_{i} \eta^{i}=h_{i} \nabla s^{i} \quad(\text { sin sumar en } i) .
\end{array}
$$

Así, la transformación de $\nabla$ es inmediata

$$
\nabla=\widehat{\mathbf{Y}}^{j} \frac{\partial}{\partial Y^{j}}=\widehat{\mathbf{Y}}^{j} \frac{\partial s^{i}}{\partial Y^{j}} \frac{\partial}{\partial s^{i}}
$$

donde aparece la definición (2.61), por lo tanto

$$
\nabla=\eta^{i} \frac{\partial}{\partial s^{i}}
$$

y usando (2.63) obtenemos

$$
\nabla=\widehat{\mathbf{s}}^{i} \frac{1}{h_{i}} \frac{\partial}{\partial s^{i}} .
$$

e) Para la transformación del operador $\nabla \cdot$ usamos la identidad

$$
\widehat{\mathbf{s}}_{i}=\frac{1}{2} \epsilon_{i j k} \widehat{\mathbf{s}}_{j} \times \widehat{\mathbf{s}}_{k},
$$

la cual es fàcil de demostrar, junto con

$$
\begin{gathered}
\nabla \cdot f \mathbf{b}=\nabla f \cdot \mathbf{b}+f \nabla \cdot \mathbf{b} \\
\nabla \cdot\left(\eta^{j} \times \eta^{k}\right)=\nabla \cdot\left(\nabla s^{j} \times \nabla s^{k}\right)=\mathbf{0}
\end{gathered}
$$

la ultima identidad se obtiene de $\nabla \cdot(\mathbf{a} \times \mathbf{b})=\mathbf{b} \cdot[\nabla \times \mathbf{a}]-\mathbf{a} \cdot[\nabla \times \mathbf{b}]$ y $\nabla \times \nabla f=\mathbf{0}$. Si tenemos

$$
\mathbf{b}=b^{i} \widehat{\mathbf{s}}_{i}
$$


entonces

$$
\begin{aligned}
\nabla \cdot \mathbf{b} & =\frac{1}{2} \epsilon_{i j k} \nabla \cdot\left(b^{i} \widehat{\mathbf{s}}_{j} \times \widehat{\mathbf{s}}_{k}\right) & & \text { [usando (2.67)] } \\
& =\frac{1}{2} \epsilon_{i j k} \nabla \cdot\left(h_{j} h_{k} b^{i} \eta^{j} \times \eta^{k}\right) & & {[\text { usando }(2.64)] } \\
& =\frac{1}{2} \epsilon_{i j k}\left[\left(\nabla h_{j} h_{k} b^{i}\right) \cdot\left(\eta^{j} \times \eta^{k}\right)+\mathbf{0}\right] & & {[\text { usando }(2.68),(2.69)] } \\
& =\frac{1}{2} \epsilon_{i j k} \nabla\left(h_{j} h_{k} b^{i}\right) \cdot\left(\frac{\widehat{\mathbf{s}}^{j}}{h_{j}} \times \frac{\widehat{\mathbf{s}}^{k}}{h_{k}}\right) & & {[\text { usando }(2.63)] } \\
& =\frac{1}{h_{j} h_{k}} \nabla\left(h_{j} h_{k} b^{i}\right) \cdot \widehat{\mathbf{s}}^{i} & & {[\text { usando }(2.67)](\sin \text { sumar en } j, k) } \\
& =\frac{1}{h_{j} h_{k} h_{i}} \frac{\partial}{\partial s^{i}}\left(h_{j} h_{k} b^{i}\right) & & \text { [usando }(2.66)](\sin \text { sumar en } j, k) .
\end{aligned}
$$

Por lo tanto

$$
\begin{aligned}
\nabla \cdot\left(b^{i} \widehat{\mathbf{s}}_{i}\right) & =\frac{1}{h_{j} h_{k} h_{i}} \frac{\partial}{\partial s^{i}}\left(h_{j} h_{k} b^{i}\right) \quad(\text { sin sumar en } j, k) \\
& =\frac{1}{h_{1} h_{2} h_{3}}\left[\frac{\partial}{\partial s^{1}} h_{2} h_{3} b^{1}+\frac{\partial}{\partial s^{2}} h_{1} h_{3} b^{2}+\frac{\partial}{\partial s^{3}} h_{1} h_{2} b^{3}\right] .
\end{aligned}
$$

La expresión anterior toma una forma compacta si usamos las componentes $b_{\eta}^{i}$ de $\mathbf{b}$ en la base $\tau_{i}$ conocidas como componentes contravariantes,

$$
\mathbf{b}=b_{\eta}^{i} \tau_{i}
$$

De acuerdo con

$$
\mathbf{b}=b^{i} \widehat{\mathbf{s}}_{i}=b_{\eta}^{i} \tau_{i}=b_{\eta}^{i} h_{i} \widehat{\mathbf{s}}_{i}
$$

la relación entre componentes físicas $b^{i}$ y contravariantes $b_{\eta}^{i}$ es

$$
\begin{aligned}
& b^{i}=h_{i} b_{\eta}^{i} \\
& b_{\eta}^{i}=\frac{b^{i}}{h_{i}} .
\end{aligned}
$$

Usando (2.72) en (2.70) se obtiene

$$
\nabla \cdot \mathbf{b}=\frac{1}{h_{1} h_{2} h_{3}}\left[\frac{\partial}{\partial s^{1}} h_{1} h_{2} h_{3} b_{\eta}^{1}+\frac{\partial}{\partial s^{2}} h_{1} h_{2} h_{3} b_{\eta}^{2}+\frac{\partial}{\partial s^{3}} h_{1} h_{2} h_{3} b_{\eta}^{3}\right]=\frac{1}{J} \frac{\partial}{\partial s^{i}} J b_{\eta}^{i} .
$$

Comparando (2.53) con (2.71) concluimos que las velocidades generalizadas $\dot{s}^{j}$ son las componentes contravariantes de $\mathbf{v}$, con lo cual (2.74) conduce a

$$
\nabla \cdot \mathbf{v}=\frac{1}{J} \frac{\partial}{\partial s^{i}} J \dot{s}^{i} .
$$

f) Para el operador $\frac{d}{d t}$ solo usamos la regla de la cadena

$$
\frac{d}{d t}=\left(\frac{\partial}{\partial t}\right)_{s}+\dot{s}^{j} \frac{\partial}{\partial s^{i}}
$$

Usando (2.55), (2.54) y (2.66) obtenemos

$$
\dot{s}^{j} \frac{\partial}{\partial s^{i}}=\frac{v^{i}}{h_{i}} \frac{\partial}{\partial s^{i}}=v^{i} \frac{1}{h_{i}} \frac{\partial}{\partial s^{i}}=\mathbf{v} \cdot \nabla
$$

con lo cual (2.76) toma la forma

$$
\frac{d}{d t}=\left(\frac{\partial}{\partial t}\right)_{s}+\frac{v^{i}}{h_{i}} \frac{\partial}{\partial s^{i}}=\left(\frac{\partial}{\partial t}\right)_{s}+\mathbf{v} \cdot \nabla
$$


g) Para la ecuación de continuidad,

$$
\frac{d \rho}{d t}+\rho \nabla \cdot \mathbf{v}=0
$$

tenemos (2.70) y (2.76) ó (2.77). Con tales expresiones obtenemos la forma flujo de la ecuación de continuidad,

$$
\left(\frac{\partial \rho}{\partial t}\right)_{s}+\nabla \cdot \rho \mathbf{v}=0
$$

Para la ecuación de momentum,

$$
\mathbf{a}+2 \boldsymbol{\Omega} \times \mathbf{v}+\boldsymbol{\Omega} \times(\boldsymbol{\Omega} \times \mathbf{R})=-\frac{1}{\rho} \nabla p+\mathbf{g}+\mathbf{f},
$$

tenemos $\mathbf{a}=a^{i} \widehat{\mathbf{s}}_{i}$ con $a^{i}$ dada por $(2.58,2.59)$, la aceleración de Coriolis esta dada por (2.52),

$$
\boldsymbol{\Omega} \times \mathbf{v}=\left|\begin{array}{ccc}
\widehat{\mathbf{s}}_{1} & \widehat{\mathbf{s}}_{2} & \widehat{\mathbf{s}}_{3} \\
\Omega^{1} & \Omega^{2} & \Omega^{3} \\
v^{1} & v^{2} & v^{3}
\end{array}\right|
$$

y la aceleración centrípeta $\boldsymbol{\Omega} \times(\boldsymbol{\Omega} \times \mathbf{R})$ se puede calcular como la de Coriolis. Para $\nabla p$ y $\mathbf{g}$ tenemos

$$
\nabla p=\widehat{\mathbf{s}}_{i} \frac{1}{h_{i}} \frac{\partial p}{\partial s^{i}}, \quad \mathbf{g}=\nabla \phi_{g}=\widehat{\mathbf{s}}_{i} \frac{1}{h_{i}} \frac{\partial \phi_{g}}{\partial s^{i}} .
$$

\subsubsection{Ecuaciones en coordenadas esféricas $\lambda, \phi, r$}

En el sistema de referencia primario $Y^{1} Y^{2} Y^{3}$ definimos las coordenadas esféricas $\lambda, \phi, r$ como se aprecia en la figura (2.5). Las ecuaciones de transformación entre las coordenadas de ambos sistemas son

$$
Y^{1}=r \cos \phi \cos \lambda \quad Y^{2}=r \cos \phi \operatorname{sen} \lambda \quad Y^{3}=r \operatorname{sen} \phi,
$$

el vector de posición está dado por

$$
\mathbf{R}=Y^{i} \widehat{\mathbf{Y}}_{i}=r\left[\cos \phi\left(\cos \lambda \widehat{\mathbf{Y}}_{1}+\operatorname{sen} \lambda \widehat{\mathbf{Y}}_{2}\right)+\operatorname{sen} \phi \widehat{\mathbf{Y}}_{3}\right] .
$$

Con la definición siguiente

$$
s^{1} \equiv \lambda \quad s^{2} \equiv \phi \quad s^{3} \equiv r
$$

la base covariante $\tau_{i}(2.39)$ esta dada por

$$
\tau_{\lambda}=\frac{\partial \mathbf{R}}{\partial \lambda}=h_{\lambda} \widehat{\lambda}, \quad \tau_{\phi}=\frac{\partial \mathbf{R}}{\partial \phi}=h_{\phi} \widehat{\phi}, \quad \tau_{r}=\frac{\partial \mathbf{R}}{\partial r}=h_{r} \widehat{\mathbf{r}},
$$

donde

$$
h_{\lambda}=r \cos \phi \quad h_{\phi}=r \quad h_{r}=1 .
$$

De (2.40) obtenemos la base ortonormal $\widehat{\mathbf{s}}^{i}$,

$$
\widehat{\mathbf{s}}^{1} \equiv \widehat{\lambda} \quad \widehat{\mathbf{s}}^{2} \equiv \widehat{\phi} \quad \widehat{\mathbf{s}}^{3} \equiv \widehat{\mathbf{r}}
$$

a saber,

$$
\left(\begin{array}{c}
\widehat{\lambda} \\
\widehat{\phi} \\
\widehat{\mathbf{r}}
\end{array}\right)=\mathbb{R}(\lambda, \phi)\left(\begin{array}{c}
\widehat{\mathbf{Y}}^{1} \\
\widehat{\mathbf{Y}}^{2} \\
\widehat{\mathbf{Y}}^{3}
\end{array}\right)
$$

donde

$$
\mathbb{R}(\lambda, \phi)=\left(\begin{array}{ccc}
-\operatorname{sen} \lambda & \cos \lambda & 0 \\
-\operatorname{sen} \phi \cos \lambda & -\operatorname{sen} \phi \operatorname{sen} \lambda & \cos \phi \\
\cos \phi \cos \lambda & \cos \phi \operatorname{sen} \lambda & \operatorname{sen} \phi
\end{array}\right)
$$


con lo cual el vector de posición (2.81) toma la forma

$$
\mathbf{R}=r \widehat{\mathbf{r}} .
$$

Para la velocidad tenemos

$$
\mathbf{V}=\mathbf{v}+\mathbf{\Omega} \times \mathbf{R}
$$

donde la velocidad relativa a la Tierra

$$
\mathbf{v}=u \widehat{\lambda}+v \widehat{\phi}+w \widehat{\mathbf{r}}
$$

tiene componentes físicas (2.54)

$$
u=\dot{\lambda} h_{\lambda}=\dot{\lambda} r \cos \phi \quad v=\dot{\phi} h_{\phi}=\dot{\phi} r \quad w=\dot{r}
$$

y las componentes contravariantes están dadas por

$$
\dot{\lambda}=\frac{u}{r \cos \phi} \quad \dot{\phi}=\frac{v}{r} \quad \dot{r}=w .
$$

Para la ecuación de continuidad

$$
\frac{d \rho}{d t}+\rho \nabla \cdot \mathbf{v}=0
$$

tenemos las ecuaciones $(2.76)$ y $(2.77)$

$$
\frac{d}{d t} \equiv \frac{\partial}{\partial t}+\dot{\lambda} \frac{\partial}{\partial \lambda}+\dot{\phi} \frac{\partial}{\partial \phi}+\dot{r} \frac{\partial}{\partial r}=\frac{\partial}{\partial t}+\frac{u}{r \cos \phi} \frac{\partial}{\partial \lambda}+\frac{v}{r} \frac{\partial}{\partial \phi}+w \frac{\partial}{\partial r}
$$

y la ecuación (2.70)

$$
\nabla \cdot \mathbf{v}=\nabla \cdot(u \widehat{\lambda}+v \widehat{\phi}+w \widehat{\mathbf{r}})=\frac{1}{r^{2} \cos \phi}\left(\frac{\partial}{\partial \lambda} r u+\frac{\partial}{\partial \phi} r \cos \phi v+\frac{\partial}{\partial r} r^{2} \cos \phi w\right) .
$$

De acuerdo con la figura (2.6), la velocidad angular terrestre es

$$
\boldsymbol{\Omega}=\Omega(\widehat{\phi} \cos \phi+\widehat{\mathbf{r}} \operatorname{sen} \phi)
$$

Para la aceleración relativa a la Tierra

$$
\mathbf{a}=a_{\lambda} \widehat{\lambda}+a_{\phi} \widehat{\phi}+a_{r} \widehat{\mathbf{r}}
$$

tenemos (2.57-2.59). Calculando

$$
\mathbb{R}^{T}=\mathbb{R} \frac{d}{d t} \mathbb{R}^{T}(\lambda, \phi)=\mathbb{R}\left(\dot{\lambda} \frac{\partial \mathbb{R}^{T}}{\partial \lambda}+\dot{\phi} \frac{\partial \mathbb{R}^{T}}{\partial \phi}\right)=\left(\begin{array}{ccc}
0 & -\frac{u \tan \phi}{r} & \frac{u}{r} \\
\frac{u \tan \phi}{r} & 0 & \frac{v}{r} \\
-\frac{u}{r} & -\frac{v}{r} & 0
\end{array}\right)
$$

se obtiene

$$
\left(\begin{array}{c}
a_{\lambda} \\
a_{\phi} \\
a_{r}
\end{array}\right)=\left(\begin{array}{c}
\dot{u} \\
\dot{v} \\
\dot{w}
\end{array}\right)+\left(\begin{array}{ccc}
0 & -\frac{u \tan \phi}{r} & \frac{u}{r} \\
\frac{u \tan \phi}{r_{u}} & 0 & \frac{v}{r} \\
-\frac{u}{r} & -\frac{v}{r} & 0
\end{array}\right)\left(\begin{array}{c}
u \\
v \\
w
\end{array}\right)
$$

o en forma escalar

$$
\begin{aligned}
& a_{\lambda}=\dot{u}-u v \frac{\tan \phi}{r}+\frac{u w}{r} \\
& a_{\phi}=\dot{v}+u^{2} \frac{\tan \phi}{r}+\frac{v w}{r} \\
& a_{r}=\dot{w}-\frac{u^{2}+v^{2}}{r}
\end{aligned}
$$




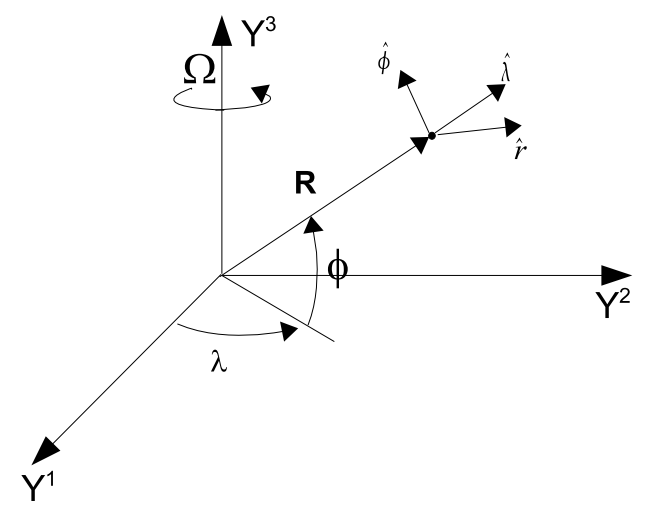

Figura 2.5: Sistema primario $Y^{i} \mathrm{y}$ sistema de coordenadas esféricas $\lambda \phi r$.

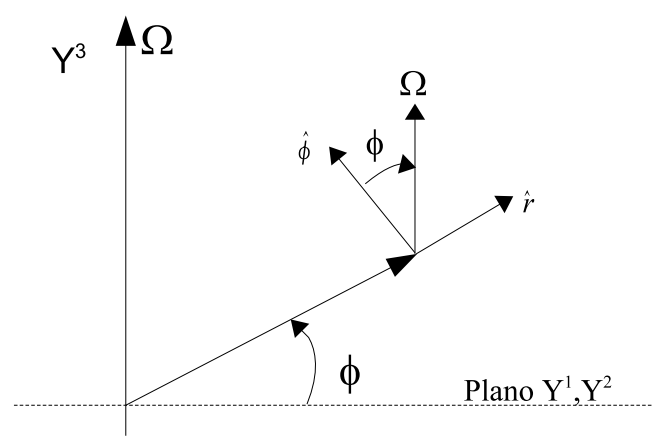

Figura 2.6: Descomposición de $\boldsymbol{\Omega}$. 
En este sistema de coordenadas, la aceleración de Coriolis tiene la siguiente expresión

$$
\begin{aligned}
\boldsymbol{\Omega} \times \mathbf{v} & =\Omega(\widehat{\phi} \cos \phi+\widehat{\mathbf{r}} \operatorname{sen} \phi) \times(u \widehat{\lambda}+v \widehat{\phi}+w \widehat{\mathbf{r}}) \\
& =\Omega(-v \operatorname{sen} \phi+w \cos \phi) \widehat{\lambda}+\Omega u \operatorname{sen} \phi \widehat{\phi}-\Omega u \cos \phi \widehat{\mathbf{r}}
\end{aligned}
$$

y la aceleración centrípeta $\boldsymbol{\Omega} \times(\boldsymbol{\Omega} \times \mathbf{R})$ se puede definirse como el gradiente de el potencial

$$
\Phi_{c}=\frac{\Omega^{2}}{2} r^{2} \cos ^{2} \phi
$$

Usando la ecuación (2.66)

$$
\nabla=\widehat{\lambda} \frac{1}{r \cos \phi} \frac{\partial}{\partial \lambda}+\widehat{\phi} \frac{1}{r} \frac{\partial}{\partial \phi}+\widehat{\mathbf{r}} \frac{\partial}{\partial r}
$$

obtenemos

$$
\boldsymbol{\Omega} \times(\boldsymbol{\Omega} \times \mathbf{R})=-\nabla \Phi_{c}=-\frac{\Omega^{2}}{2}\left[\widehat{\phi} \frac{1}{r} \frac{\partial}{\partial \phi}+\widehat{\mathbf{r}} \frac{\partial}{\partial r}\right] r^{2} \cos ^{2} \phi=\Omega^{2} r \cos \phi(\widehat{\phi} \operatorname{sen} \phi-\widehat{\mathbf{r}} \cos \phi) .
$$

En el caso particular de una Tierra esférica tenemos que el potencial gravitacional es

$$
\Phi_{g}(\mathbf{R})=g \frac{R_{e}^{2}}{r}
$$

por lo que el vector de aceleración gravitacional es

$$
\mathbf{g}(\mathbf{R})=\nabla \Phi_{g}=\left(\frac{\widehat{\lambda}}{r \cos \phi} \frac{\partial}{\partial \lambda}+\widehat{\phi} \frac{1}{r} \frac{\partial}{\partial \phi}+\widehat{\mathbf{r}} \frac{\partial}{\partial r}\right) \Phi_{g}=-g \frac{R_{e}^{2}}{r^{2}} \widehat{\mathbf{r}}
$$

Entonces las ecuaciones escalares de momentum son

$$
\begin{aligned}
\frac{d u}{d t}-\frac{u v}{r} \tan \phi+\frac{u w}{r}-2 \Omega v \operatorname{sen} \phi+2 \Omega w \cos \phi & =-\frac{1}{r \cos \phi} \frac{1}{\rho} \frac{\partial p}{\partial \lambda} \\
\frac{d v}{d t}+u^{2} \frac{\tan \phi}{r}+\frac{v w}{r}+2 \Omega u \operatorname{sen} \phi+\Omega^{2} r \cos \phi \operatorname{sen} \phi & =-\frac{1}{r} \frac{1}{\rho} \frac{\partial p}{\partial \phi} \\
\frac{d w}{d t}-\frac{u^{2}+v^{2}}{r}-2 \Omega u \cos \phi-\Omega^{2} r \cos ^{2} \phi & =-\frac{1}{\rho} \frac{\partial p}{\partial r}-g \frac{R_{e}^{2}}{r^{2}}
\end{aligned}
$$

Para simplificar la ecuación (2.99) se considera que los términos con $\Omega^{2}$ son pequeños comparados con los demás. Además, también se considera que la única fuerza de volumen de importancia es la gravedad.

\subsubsection{Ecuaciones en coordenadas curvilíneas esféricas $x_{s} y_{s} z_{s}$}

Las coordenadas curvilíneas esféricas se definen por

$$
x_{s}=a \cos \phi\left(\lambda-\lambda_{c}\right) \quad y_{s}=a\left(\phi-\phi_{c}\right) \quad z_{s}=r-a .
$$

Estas coordenadas constituyen básicamente un reescalamiento de las coordenadas esféricas $\lambda \phi r$ y miden el desplazamiento de una partícula a partir de un punto de referencia con coordenadas esféricas $\left(\lambda_{c}, \phi_{c}, r=a\right)$ como se aprecia en la figura (2.7).

Despejando obtenemos

$$
\lambda=\frac{x_{s}}{a \cos \left[\frac{y_{s}}{a}+\phi_{c}\right]}+\lambda_{c} \quad \phi=\frac{y_{s}}{a}+\phi_{c} \quad r=z_{s}+a
$$

donde

$$
a=\text { radio de una esfera que aproxime a la Tierra. }
$$



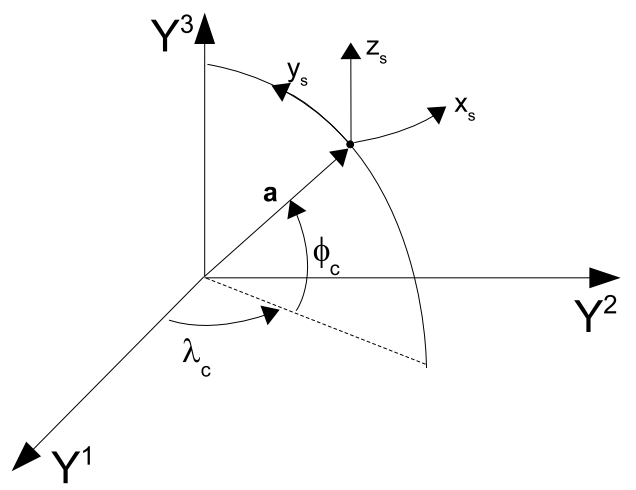

Figura 2.7: Coordenadas curvilíneas esféricas $x_{s}, y_{s}, z_{s}$.

Los vectores base unitarios coinciden con aquellos en coordenadas esféricas,

$$
\widehat{\mathbf{x}}_{s}=\widehat{\lambda} \quad \widehat{\mathbf{y}}_{s}=\widehat{\phi} \quad \widehat{\mathbf{z}}_{s}=\widehat{\mathbf{r}}
$$

y los factores métricos correspondientes son

$$
h_{x}=\frac{r \cos \phi}{a \cos \phi_{c}} \quad h_{y}=\frac{r}{a} \quad h_{z}=1
$$

Para obtener expresiones en el sistema $x_{s} y_{s} z_{s}$ basta con usar las expresiones en coordenadas esféricas y las relaciones

$$
\begin{aligned}
\frac{\partial}{\partial \lambda} & =\frac{\partial x_{s}}{\partial \lambda} \frac{\partial}{\partial x_{s}}= & a \cos \phi_{c} & \frac{\partial}{\partial x_{s}} \\
\frac{\partial}{\partial \phi} & =\frac{\partial y_{s}}{\partial \phi} \frac{\partial}{\partial y_{s}}= & a & \frac{\partial}{\partial y_{s}} \\
\frac{\partial}{\partial r} & =\frac{\partial z_{s}}{\partial r} \frac{\partial}{\partial z_{s}}= & & \frac{\partial}{\partial z_{s}}
\end{aligned} .
$$

Por ejemplo, para el gradiente

$$
\nabla=\widehat{\lambda} \frac{1}{r \cos \phi} \quad \frac{\partial}{\partial \lambda}+\widehat{\phi} \frac{1}{r} \frac{\partial}{\partial \phi}+\widehat{\mathbf{r}} \frac{\partial}{\partial r}=\widehat{\mathbf{x}}_{s} \frac{a \cos \phi_{c}}{r \cos \phi} \frac{\partial}{\partial x_{s}}+\widehat{\mathbf{y}} \frac{a}{r} \frac{\partial}{\partial y_{s}}+\widehat{\mathbf{z}}_{s} \frac{\partial}{\partial z_{s}}
$$

De acuerdo con (2.85) y (2.105) el vector de posición es

$$
\mathbf{R}=\left(z_{s}+a\right) \widehat{\mathbf{r}}
$$

y la identidad (2.106) implica que las componentes físicas de la velocidad relativa a la Tierra

$$
\mathbf{v}=v_{s} \widehat{\mathbf{x}}_{s}+v_{s} \widehat{\mathbf{y}}_{s}+v_{s} \widehat{\mathbf{z}}_{s}
$$

coinciden con aquellas en coordenadas esféricas

$$
u_{s}=u_{a}=v_{a} \quad w_{s}=w_{a} .
$$

Las relaciones entre velocidades generalizadas son

$$
\begin{aligned}
\dot{x}_{s}=a \cos \phi_{c} \dot{\lambda} & =\frac{a \cos \phi_{c}}{r \cos \phi} u_{s} \\
\dot{y}_{s}=a \dot{\phi} & =\frac{a}{r} v_{s} \\
\dot{z}_{s}= & \dot{r}=w_{s}
\end{aligned}
$$


En la ecuación de continuidad

$$
\frac{d \rho}{d t}+\rho \nabla \cdot \mathbf{v}=0
$$

tenemos

$$
\begin{aligned}
\frac{d}{d t} & =\frac{\partial}{\partial t}+\dot{x_{s}} \frac{\partial}{\partial x_{s}}+\dot{y_{s}} \frac{\partial}{\partial y_{s}}+\dot{z_{s}} \frac{\partial}{\partial z_{s}} \\
& =\frac{\partial}{\partial t}+\frac{a \cos \phi_{c}}{r \cos \phi} u_{s} \frac{\partial}{\partial x_{s}}+\frac{a}{r} v_{s} \frac{\partial}{\partial y_{s}}+w \frac{\partial}{\partial z_{s}}
\end{aligned}
$$

y para $\nabla \cdot \mathbf{v}$ sustituimos $r=z_{s}+a$ en lo siguiente

$$
\begin{aligned}
\nabla \cdot \mathbf{v} & =\frac{1}{r^{2} \cos \phi}\left[a \cos \phi_{c} \frac{\partial}{\partial x_{s}} r u_{s}+a \frac{\partial}{\partial y_{s}} r \cos \phi v_{s}+\frac{\partial}{\partial z_{s}} r^{2} \cos \phi w_{s}\right] \\
& =\frac{a \cos \phi_{c}}{r \cos \phi} \frac{\partial u_{s}}{\partial x_{s}}+\frac{a}{r \cos \phi} \frac{\partial\left(v_{s} \cos \phi v_{s}\right)}{\partial y_{s}}+\frac{\partial w_{s}}{\partial z_{s}}+\frac{2}{r} w_{s} .
\end{aligned}
$$

En el caso particular de una Tierra esférica tenemos $r=z_{s}+a$ las ecuaciones escalares de momentum son

$$
\begin{aligned}
\frac{d u_{s}}{d t}-\frac{u_{s} v_{s}}{r} \tan \phi+\frac{u_{s} w_{s}}{r}-2 \Omega v_{s} \operatorname{sen} \phi+2 \Omega w_{s} \cos \phi & =-\frac{a \cos \phi_{c}}{r \cos \phi} \frac{1}{\rho} \frac{\partial p}{\partial x_{s}} \\
\frac{d v_{s}}{d t}+u_{s}^{2} \frac{\tan \phi}{r}+\frac{v_{s} w_{s}}{r}+2 \Omega u_{s} \operatorname{sen} \phi+\Omega^{2} r \cos \phi \operatorname{sen} \phi & =-\frac{a}{r} \frac{\partial p}{\partial y_{s}} \\
\frac{d w_{s}}{d t}-\frac{u_{s}^{2}+v_{s}^{2}}{r}-2 \Omega u_{s} \cos \phi-\Omega^{2} r \cos ^{2} \phi & =-\frac{1}{\rho} \frac{\partial p}{\partial z_{s}}-g \frac{R_{e}^{2}}{r^{2}}
\end{aligned}
$$

\subsection{Ecuación de Bernoulli en un sistema fijo a la Tierra}

Consideremos los sistemas de referencia inercial $X^{1}, X^{2}, X^{3}$ y primario $X=Y^{1}, Y=Y^{2}, Z=Y^{3}$. La relación entre las velocidades $\mathbf{V}$ y $\mathbf{v}$ es

$$
\mathbf{V}=\mathbf{v}+\mathbf{\Omega} \times \mathbf{R}
$$

donde $\mathbf{v}$ es la velocidad relativa a la Tierra. Para obtener la ecuación de Bernoulli en términos de cantidades relativas a la Tierra conviene tener presente los resultados siguientes.

Proposición 2.1 El término $\boldsymbol{\Omega} \times \mathbf{R}$ no puede representarse como el gradiente de un campo escalar.

En efecto, en el sistema de referencia primario tenemos $\boldsymbol{\Omega}=\Omega \widehat{\mathbf{Z}}$ y por tanto

$$
\boldsymbol{\Omega} \times \mathbf{R}=\Omega \widehat{\mathbf{Z}} \times(X \widehat{\mathbf{X}}+Y \widehat{\mathbf{Y}}+Z \widehat{\mathbf{Z}})=\Omega(-Y \widehat{\mathbf{X}}+X \widehat{\mathbf{Y}})
$$

Supongamos que existe un potencial $\Phi_{\Omega}$ que satisface la relación $\Omega \times \mathbf{R}=\nabla \Phi_{\Omega}$ la cual equivale al par de ecuaciones

$$
\frac{\partial \Phi_{\Omega}}{\partial X}=-\Omega Y \quad \frac{\partial \Phi_{\Omega}}{\partial Y}=\Omega X .
$$

Integrando la primera ecuación

$$
\Phi_{\Omega}=-\Omega Y X+f(Y)
$$

y derivando la ecuación resultante con respecto a $Y$

$$
\frac{\partial \Phi_{\Omega}}{\partial Y}=-\Omega X+\frac{d f(Y)}{d Y}
$$

se llega a una contradicción

$$
\frac{d f(Y)}{d Y}=2 \Omega X
$$

Corolario 2.1 . Los flujos $\mathbf{V}$ y $\mathbf{v}$ no pueden ser simultáneamente potenciales. 
En efecto si tenemos $\mathbf{V}=\nabla \Phi_{V}, \mathbf{v}=\nabla \Phi_{v}$, entonces $\mathbf{V}-\mathbf{v}=\nabla\left(\Phi_{V}-\Phi_{v}\right)=\mathbf{\Omega} \times \mathbf{R}$ lo que contradice la proposición anterior.

En ausencia de fuerzas distintas a la presión, la ecuación de movimiento [ec. (1.64)] de una partícula respecto al sistema $x^{1}, x^{2}, x^{3}$ es

$$
\mathbf{a}+2 \boldsymbol{\Omega} \times \mathbf{v}+\boldsymbol{\Omega} \times(\boldsymbol{\Omega} \times \mathbf{R})=-\frac{1}{\rho} \nabla p+\mathbf{g} .
$$

En términos de los potenciales asociados a la aceleración centrípeta y la aceleración gravitacional,

$$
\boldsymbol{\Omega} \times(\boldsymbol{\Omega} \times \mathbf{R})=-\nabla \Phi_{c} \quad \mathbf{g}=\nabla \Phi_{g}
$$

podemos escribir

$$
\mathbf{a}+2 \mathbf{\Omega} \times \mathbf{R}=-\frac{1}{\rho} \nabla p+\nabla \Phi
$$

donde definimos

$$
\Phi \equiv \Phi_{g}+\Phi_{c}
$$

La aceleración a, relativa a la Tierra, puede reescribirse como sigue

$$
\mathbf{a}=\frac{d v^{i}}{d t} \widehat{\mathbf{x}}_{i}=\frac{\partial v^{i}}{\partial t} \widehat{\mathbf{x}}_{i}+v^{j} \frac{\partial v^{i}}{\partial x^{j}} \widehat{\mathbf{x}}_{i}=\frac{\partial v^{i}}{\partial t} \widehat{\mathbf{x}}_{i}+(\mathbf{v} \cdot \nabla) v^{i} \widehat{\mathbf{x}}_{i}=\frac{\partial v^{i}}{\partial t} \widehat{\mathbf{x}}_{i}+(\mathbf{v} \cdot \nabla) \mathbf{v}
$$

donde

$$
\nabla=\widehat{\mathbf{x}}^{j} \frac{\partial}{\partial x^{j}}
$$

sustituyendo lo anterior en la ecuación de movimiento obtenemos

$$
\frac{\partial v^{i}}{\partial t} \widehat{\mathbf{x}}_{i}+(\mathbf{v} \cdot \nabla) \mathbf{v}+2 \boldsymbol{\Omega} \times \mathbf{v}=-\frac{1}{\rho} \nabla p+\nabla \phi .
$$

Es fácil ver que tiene lugar la identidad

$$
(\mathbf{v} \cdot \nabla) \mathbf{v}=\nabla \frac{v^{2}}{2}-\mathbf{v} \times(\nabla \times \mathbf{v})
$$

con la cual (2.112) toma la forma

$$
\frac{\partial v^{i}}{\partial t} \widehat{\mathbf{x}}_{i}+\frac{1}{\rho} \nabla p+\nabla\left[\frac{v^{2}}{2}-\Phi\right]=\mathbf{v} \times[2 \boldsymbol{\Omega}+\nabla \times \mathbf{v}]
$$

Si $x^{i}$ son las coordenadas instantáneas (o Eulerianas) de una partícula, las coordenadas $\left.x_{0}^{i} \equiv x^{i}\right|_{t_{0}}$ en un instante dado $t_{0}$ son las correspondientes coordenadas Lagrangianas. Si conocemos las componentes $v^{i} \equiv \dot{x}^{i}$ de la velocidad $\mathbf{v} \equiv v^{i} \widehat{\mathbf{x}}_{i}$ en su forma Euleriana $v^{i}\left(t, x^{i}\right)$, podemos recuperar la forma en la que las coordenadas $x^{i}$ dependen de $t$ y $x_{0}^{i}$ resolviendo el sistema de ecuaciones diferenciales ordinarias

$$
\frac{d x^{i}}{d t}=v^{i}\left(t, x^{i}\right) \quad \text { con } i=1,2,3
$$

sujeto a la condición inicial

$$
\left.x^{i}\right|_{t_{0}}=x_{0}^{i}
$$

Si el tiempo aparece explícitamente en $v^{i}\left(t, x^{i}\right)$, entonces, en general, partículas que pasen por un mismo punto $x_{0}^{i}$ en instantes diferentes, tendrán trayectorias diferentes, mientras que en el caso autónomo o estacionario $v^{i}=v^{i}\left(x^{i}\right)$, las trayectorias de partículas que pasan por el mismo punto coincidirán.

Dado que estamos interesados en la descripción respecto a la Tierra diremos que un flujo es estacionario si las propiedades medidas en un punto fijo respecto a la Tierra, no cambian con el tiempo. Por ejemplo, si el campo $\mathbf{v}$ es estacionario, la forma Euleriana de sus componentes $v^{i}$ no depende explícitamente del tiempo,

$$
\frac{\partial v^{i}}{\partial t}=0
$$


de manera que

$$
\frac{d}{d t} v^{i}=\frac{d}{d t} v^{i}\left(x^{1}, x^{2}, x^{3}\right)=\frac{d x^{j}}{d t} \frac{\partial v^{i}}{\partial x^{j}}=\mathbf{v} \cdot \nabla v^{i}
$$

Sean $\mathcal{Q}_{0}\left(t, x_{0}^{i}\right)$ y $\mathcal{Q}\left(t, x^{i}\right)$ a las formas Lagrangiana y Euleriana, respectivamente, de una propiedad $\mathcal{Q}$ de una partícula. Tenemos

$$
\mathcal{Q}_{0}\left(t, x_{0}^{i}\right)=\mathcal{Q}\left(t, x^{i}\right)
$$

y derivando obtenemos

$$
\frac{d}{d t} \mathcal{Q}_{0}=\frac{\partial \mathcal{Q}}{\partial t}+\mathbf{v} \cdot \nabla \mathcal{Q}
$$

Si suponemos que (i) el flujo es estacionario y (ii) el valor de $\mathcal{Q}$ dentro de cada partícula de fluido no cambia con el tiempo, entonces

$$
\frac{d}{d t} \mathcal{Q}_{0}=\frac{\partial \mathcal{Q}}{\partial t}=0
$$

con lo cual (2.114) se reduce a

$$
\mathbf{v} \cdot \nabla \mathcal{Q}(\mathbf{r})=0 \quad \text { para cada } \mathbf{r} .
$$

Recíprocamente, si la ecuación (2.115) se cumple y el flujo es estacionario, entonces la propiedad $\mathcal{Q}$ no cambia en el interior de una partícula mientras ésta se mueve o, equivalentemente, $\mathcal{Q}$ tiene un valor constante sobre la trayectoria de cada partícula,

$$
\left.\mathcal{Q}\right|_{\mathbf{r}_{0}}=\left.\mathcal{Q}\right|_{\mathbf{r}}
$$

Consideremos un flujo potencial $\mathbf{v}=\nabla \Phi_{v}$ con densidad constante $\rho=\rho_{0}$. Entonces $\nabla \times \mathbf{v}=\mathbf{0} \mathrm{y}$

$$
\widehat{\mathbf{x}}_{i} \frac{\partial v^{i}}{\partial t}=\widehat{\mathbf{x}}_{i} \frac{\partial}{\partial t} \frac{\partial \Phi_{v}}{\partial x^{i}}=\widehat{\mathbf{x}}_{i} \frac{\partial}{\partial x^{i}} \frac{\partial \Phi_{v}}{\partial t}=\nabla \frac{\partial \Phi_{v}}{\partial t} .
$$

con lo cual la ecuación (2.113) toma la forma

$$
\nabla\left[\frac{\partial \Phi_{v}}{\partial t}+\frac{p}{\rho_{0}}+\frac{v^{2}}{2}-\Phi\right]=2 \mathbf{v} \times \boldsymbol{\Omega} .
$$

Multiplicando por $\mathbf{v} \cdot$ se obtiene

$$
\mathbf{v} \cdot \nabla\left[\frac{\partial \Phi_{v}}{\partial t}+\frac{p}{\rho_{0}}+\frac{v^{2}}{2}-\Phi\right]=0 .
$$

Dado que el flujo puede ser no estacionario la función siguiente

$$
E \equiv \frac{\partial \Phi_{v}}{\partial t}+\frac{p}{\rho_{0}}+\frac{v^{2}}{2}-\Phi
$$

puede depender explícitamente de $t$,

$$
E=E(t, \mathbf{r})
$$

con lo cual tenemos

$$
\frac{d E}{d t}=\frac{\partial E}{\partial t}+\mathbf{v} \cdot \nabla E
$$

pero $\mathbf{v} \cdot \nabla E=0$ por $(2.117)$ por tanto

$$
\frac{d E}{d t}=\frac{\partial E}{\partial t}
$$

de donde no podemos inferir que el valor de $E$ es constante sobre la trayectoria de una partícula. Esto parece contradecir a las ecuaciones de Bernoulli obtenidas en un sistema de referencia inercial, pero la contradicción sólo es aparente ya que si $\mathbf{v}$ es potencial el campo $\mathbf{V}$ dado por $\mathbf{V}=\mathbf{v}+\boldsymbol{\Omega} \times \mathbf{R}$ no es potencial . Si en adición a las hipótesis iniciales supondremos que el flujo es estacionario podemos concluir que la función

$$
E(\mathbf{r})=\frac{p(\mathbf{r})}{\rho_{0}}+\frac{v^{2}(\mathbf{r})}{2}-\Phi(\mathbf{r})
$$


es una constante de movimiento para cada partícula, es decir, es constante sobre la trayectoria de cada partícula.

El ejemplo anterior muestra que la hipótesis de flujo potencial no permite simplificar la ecuación de movimiento (2.113) ya que sobrevive el término no-inercial $\boldsymbol{\Omega} \times \mathbf{v}$ el cual se anuló multiplicando (2.116) por $\mathbf{v} \cdot$, pero tal operación también anula al término $\mathbf{v} \times \nabla \times \mathbf{v}$ sin necesidad de suponer que el flujo es potencial lo que nos lleva a reformular el ejemplo anterior como sigue.

\subsubsection{Flujo isocórico}

Si el flujo es estacionario en todas sus propiedades y la densidad es constante $\rho=\rho_{0}$, la ecuación (2.113) toma la forma

$$
\nabla\left[\frac{p}{\rho_{0}}+\frac{v^{2}}{2}-\Phi\right]=\mathbf{v} \times[-2 \boldsymbol{\Omega} \times \mathbf{v}+\nabla \times \mathbf{v}]
$$

Multiplicando por $\mathbf{v} \cdot$, se obtiene

$$
\mathbf{v} \cdot \nabla\left[\frac{p}{\rho_{0}}+\frac{v^{2}}{2}-\Phi\right]=0
$$

de donde se concluye que la función $E(\mathbf{r})$ (2.118) es constante sobre la trayectoria de cada partícula (proposición 5).

Para obtener otra formulación de la ecuación de Bernoulli sustituyamos la hipótesis de densidad constante por la de flujo adiabático o isoentrópico. De acuerdo con la hipótesis de equilibrio local, la segunda Ley de la Termodinámica en su forma (1.32) es válida, sólo que ahora las funciones termodinámicas son funciones de las coordenadas $x^{i}$ relativas a la Tierra y del tiempo $t$. Por ejemplo, para la entalpía tenemos $h=h\left(t, x^{i}\right)$ y

$$
d h=\frac{\partial h}{\partial t} d t+\frac{\partial h}{\partial x^{i}} d x^{i}
$$

Usando la expresión de $\nabla$ en la base $\widehat{\mathbf{x}}_{i}$ y $d \mathbf{r} \equiv \widehat{\mathbf{x}}_{i} d x^{i}$ podemos escribir

$$
d h=\frac{\partial h}{\partial t} d t+\nabla h \cdot d \mathbf{r}
$$

Si el flujo es estacionario tenemos simplemente $d h=\nabla h \cdot d \mathbf{r}$.

\subsubsection{Flujo isoentrópico}

Consideremos un flujo estacionario e isoentrópico. Para un flujo estacionario la ecuación (2.113) se reduce $\mathrm{a}$

$$
\frac{1}{\rho} \nabla p+\nabla\left[\frac{v^{2}}{2}-\Phi\right]=\mathbf{v} \times[2 \boldsymbol{\Omega}+\nabla \times \mathbf{v}] .
$$

Si el flujo de calor es nulo en cada punto del fluido la segunda ley (2.114) se reduce a $d h=d p / \rho$ que equivale a $\nabla h=\frac{1}{\rho} \nabla p$ con lo cual se obtiene

$$
\nabla\left[h+\frac{v^{2}}{2}-\Phi\right]=\mathbf{v} \times[2 \boldsymbol{\Omega}+\nabla \times \mathbf{v}]
$$

Multiplicando por $\mathbf{v} \cdot$ se concluye que la función

$$
E_{h}(\mathbf{r}) \equiv h(\mathbf{r})+\frac{v^{2}(\mathbf{r})}{2}-\Phi(\mathbf{r})
$$

tiene una valor constante sobre la trayectoria de cada partícula

$$
\left[h+\frac{v^{2}}{2}-\Phi\right]_{\mathbf{r}_{0}}=\left[h+\frac{v^{2}}{2}-\Phi\right]_{\mathbf{r}}
$$




\subsubsection{Flujo isotérmico}

Consideremos un flujo estacionario de gas ideal isotérmico, es decir, con temperatura constante, flujo que puede ser no-potencial. Usando la ecuación de estado $p=\mathcal{R} T \rho(T=$ cte.) obtenemos

$$
\frac{1}{\rho} \nabla p=\frac{\mathcal{R} T}{p} \nabla p=\mathcal{R} T \nabla \ln p=\nabla \mathcal{R} T \ln p
$$

y (2.113) toma la forma

$$
\nabla\left[\mathcal{R} T \ln p+\frac{v^{2}}{2}-\Phi\right]=\mathbf{v} \times[2 \boldsymbol{\Omega}+\nabla \times \mathbf{v}] .
$$

Multiplicando por v · concluimos que la función

$$
E(\mathbf{r})=\mathcal{R} T \ln p(\mathbf{r})+\frac{v^{2}(\mathbf{r})}{2}-\Phi(\mathbf{r})
$$

tiene un valor constante sobre la trayectoria de cada partícula,

$$
\left[\mathcal{R} T \ln p+\frac{v^{2}}{2}-\Phi\right]_{\mathbf{r}_{1}}=\left[\mathcal{R} T \ln p+\frac{v^{2}}{2}-\Phi\right]_{\mathbf{r}_{2}}
$$

\subsection{El problema primario $\nabla \cdot \rho_{0} \mathbf{v}=0$}

El conjunto de ecuaciones de Poisson (1.39-2.121) junto con la ecuación de Bernoulli (2.120) es importante ya que permite obtener las funciones termodinámicas de cada partícula de fluido a partir del campo de velocidad $\mathbf{v}(\mathbf{r})$ y dos valores $p_{00}$ y $T_{00}$ en un punto arbitrario $\mathbf{r}_{00}$.

Proposición 2.2 Si conocemos el campo de velocidad $\mathbf{v}(\mathbf{r})$ de un flujo ideal, estacionario e isoentrópico en cada punto $\mathbf{r}$ del espacio y un par de valores $\left(p_{00}, T_{00}\right)$, ó $\left(\rho_{00}, T_{00}\right)$ ó $\left(p_{00}, \rho_{00}\right)$, entonces podemos calcular cada variable termodinámica en cada punto del espacio.

Un resultado similar puede enunciarse en el caso de un flujo ideal y estacionario con densidad constante ó isotérmico. Esto enfatiza el bien conocido hecho en hidrodinámica que el campo de velocidad $\mathbf{v}$ es una de las variables fundamentales. El problema es, entonces, el cálculo de v. En todo este capítulo no hemos considerado la ecuación de continuidad

$$
\frac{d \rho}{d t}+\rho \nabla \cdot \mathbf{v}=0 \quad \text { ó } \quad \frac{\partial \rho}{\partial t}+\nabla \cdot \rho \mathbf{v}=0 .
$$

Si el flujo es estacionario se reduce a

$$
\nabla \cdot \rho \mathbf{v}=0
$$

y si consideremos que las variaciones de la densidad son pequeñas con respecto a un valor de referencia $\rho_{0}(\mathbf{r})$, podemos considerar la ecuación

$$
\nabla \cdot \rho_{0} \mathbf{v}=0
$$

que debe resolverse con la condición de frontera

$$
\mathbf{v} \cdot \mathbf{n}=0
$$

sobre las fronteras sólidas, donde $\mathbf{n}$ es un vector normal a dichas fronteras. Afortunadamente podemos obtener una solución completa de las ecuaciones hidrodinámicas. $(2.123,2.124)$ en regiones con una simetría razonable. 


\section{7. $\quad$ Modelo elipsoidal terrestre}

Los datos de elevación del terreno (la topografía) están definidos con respecto a un elipsoide como superficie de referencia [14]. Consideremos, por ejemplo, el elipsoide de referencia WGS84 (abreviatura de "World Geodetic System 1984") con ejes

$$
a=6378,137 \mathrm{~km} \quad b=6356,753 \mathrm{~km},
$$

donde consideramos que el radio ecuatorial del $W G S 84$ coincide con el de la esfera $\mathcal{S}_{a}$. Aunque la diferencia relativa entre $a$ y $b$ es muy pequeña,

$$
\frac{a-b}{a}=3 \times 10^{-3},
$$

la diferencia absoluta

$$
a-b=21,384 \mathrm{~km}
$$

tiene el espesor promedio de la troposfera. Es por esta razón que el modelo esférico no es el mejor modelo para estudiar la dinámica atmosférica a nivel global.

Algunos autores como Belinskii [1] o Holton [13] han señalado que la aceleración centrípeta se puede incluir en la definición de un modelo elipsoidal terrestre para que tal superficie coincida con una superficie de presión constante, sin dar detalles del cálculo correcto del modelo elipsoidal y su potencial gravitacional. Después de una discusión sobre el asunto Belinskii y Holton usan las ecuaciones de conservación en coordenadas esféricas (1.4), que omiten los términos con $\Omega^{2}$, sin estimar su región de validez y como si fueran adecuadas a escala planetaria.

La verdadera superficie terrestre, conocida como geoide, se define a partir de una superficie de referencia, la cual a su vez se define a partir de la fuerza que actúa sobre un objeto localizado sobre dicha superficie de referencia. De acuerdo con la 2a. ley de Newton la aceleración de una partícula con respecto al sistema de referencia primario $Y^{1}, Y^{2}, Y^{3}$ fijo a la tierra es

$$
\mathbf{a}=\frac{1}{m} \mathbf{F}-2 \boldsymbol{\Omega} \times \mathbf{v}+\mathbf{g}-\boldsymbol{\Omega} \times(\boldsymbol{\Omega} \times \mathbf{R})
$$

donde $\mathbf{F}$ es la resultante de la fuerzas distintas a la aceleración gravitacional $\mathbf{g}$, las fuerzas inerciales de Coriolis y centrípeta. La aceleración $\mathbf{g}$ está dada por

$$
\mathrm{g}=\nabla G \mathcal{V}
$$

donde $\mathcal{V}$ el potencial gravitacional

$$
\mathcal{V}(\mathbf{R})=\int_{\Sigma} \frac{d M\left(\mathbf{R}^{\prime}\right)}{\left\|\mathbf{R}-\mathbf{R}^{\prime}\right\|} .
$$

La aceleración centrípeta puede escribirse como el gradiente de una función,

$$
-\boldsymbol{\Omega} \times(\boldsymbol{\Omega} \times \mathbf{R})=\nabla \frac{1}{2} \Omega^{2}\left(X^{2}+Y^{2}\right),
$$

en esta forma podemos reescribir

$$
\mathbf{g}-\mathbf{\Omega} \times(\boldsymbol{\Omega} \times \mathbf{R})=\nabla \mathcal{W}
$$

donde definimos el potencial gravitatorio

$$
\mathcal{W}=G \mathcal{V}+\frac{1}{2} \Omega^{2}\left(X^{2}+Y^{2}\right) .
$$

En la definición formal del modelo matemático terrestre se considera que el eje $Z$ del sistema fijo a la tierra coincide con el eje de rotación terrestre [5], [14].

Definición. El modelo matemático terrestre o superficie de referencia es un elipsoide $\mathcal{E}$ con las propiedades siguientes: (i) cuya velocidad angular coincide con la de la tierra $\boldsymbol{\Omega}=\Omega \hat{\mathbf{Z}}$, (ii) su ecuación cartesiana tiene la forma

$$
\frac{X^{2}}{a^{2}}+\frac{Y^{2}}{c^{2}}+\frac{Z^{2}}{b^{2}}=1
$$


(iii) contiene una masa $M$ que genera un potencial gravitatorio $\mathcal{W}, \mathrm{y}$ (iv) los parámetros $a, b, c$ son tales que la función potencial $\mathcal{W}$ es constante en $\mathcal{E}$.

Nota. Durante el siglo XIX se comenzaron a proponer modelos elipsoidales de la tierra y a la fecha hay numerosos elipsoides de referencia $\mathcal{E}$ definidos para aproximar la verdadera superficie terrestre (geoide) en alguna región de interés. Sin embargo, los satélites artificiales han proporcionado datos suficientes para definir un modelo elipsoidal global con parámetros $a, b, G M$ y $\Omega$ más precisos. Por ejemplo, los parámetros del WGS84,

$$
a=6378137 \mathrm{~m} \quad b=6356752,314 \underline{24} \mathrm{~m}
$$

y los del Geodetic Reference System 80 (GRS80),

$$
a=6378137 \mathrm{~m} \quad b=6356752,314 \underline{14} \mathrm{~m}
$$

son los mismos para cualquier aplicación en modelación atmosférica [14]. Por tal razón, al hablar del elipsoide de referencia $\mathcal{E}$ nos referiremos al mejor elipsoide definido con la información disponible en el momento de leer este reporte.

De acuerdo con el desarrollo multipolar

$$
\left\|\mathbf{R}-\mathbf{R}^{\prime}\right\|^{-1}=r^{-1}\left(1+\zeta^{2}-2 \zeta \chi\right)^{-1 / 2}=r^{-1} \sum_{n=0}^{\infty} P_{n}(\chi) \zeta^{n},
$$

donde $P_{n}$ son los polinomios de Legendre y

$$
\zeta=\frac{r^{\prime}}{r} \quad \chi=\frac{\mathbf{R} \cdot \mathbf{R}^{\prime}}{r r^{\prime}},
$$

el potencial $\mathcal{V}$ tiene la forma

$$
\mathcal{V}(\mathbf{R})=r^{-1} M+O\left(r^{-2}\right)
$$

con la cual se obtiene el comportamiento asintótico

$$
\lim _{r \rightarrow \infty} r \mathcal{V}(\mathbf{R})=M \text {. }
$$

Además, $\mathcal{V}$ es una función armónica en la región exterior al elipsoide $\mathcal{E}$, es decir, satisface la ecuación de Laplace

$$
\nabla^{2} \mathcal{V}=0 \quad \text { para }(X, Y, Z) \text { fuera de } \mathcal{E}
$$

Podemos resumir la definición del elipsoide de referencia $\mathcal{E}$ como la superficie (i) en la cual $\mathcal{W}$ es una constante $[\mathcal{W}]_{\mathcal{E}}$

$$
\mathcal{W}=G \mathcal{V}+\frac{1}{2} \Omega^{2}\left(X^{2}+Y^{2}\right)=[\mathcal{W}]_{\mathcal{E}} \quad \text { para }(X, Y, Z) \text { en } \mathcal{E}
$$

(ii) $\mathcal{V}$ satisface $(2.125)$ y (2.126). Existe una expresión de $\mathcal{V}$ que satisface estas condiciones para un conjunto dado de parámetros $\{a, b, c, \Omega, M\}$ en términos de integrales elípticas. Por otro lado, la información del campo gravitacional terrestre sugiere que el geoide puede aproximarse por un elipsoide de revolución $\mathcal{E}$ con $a=c$.

En lo que resta del trabajo consideramos, por simplicidad, que la Tierra es una esfera aunque el planteamiento es válido para un modelo elipsoidal.

Una deduccion detallada de las ecuaciones de movimiento considerando el campo gravitacional y la geometría de un modelo elipsoidal terrestre se da en [26]. 


\section{Capítulo 3}

\section{Simplificación de las ecuaciones básicas}

El objetivo primario de éste capítulo es obtener simplificaciones de las ecuaciones de movimiento para flujos atmosféricos con escalas espaciales horizontales del orden de $10^{3} \mathrm{~km}$.

\subsection{Análisis de escalas}

Hasta aquí se han desarrollado de manera abstracta las ecuaciones que gobiernan el movimiento de las masas de aire alrededor del globo terráqueo. En teoría, podemos obtener con las ecuaciones (2.111) las variables atmosféricas en latitudes medias y bajas del planeta sobre alguna región del tamaño que queramos. Sin embargo la complejidad y el gran tamaño de la atmósfera hacen de estas ecuaciones un modelo poco práctico para describir la mayoría de los fenómenos atmosféricos. Por ejemplo, no es lo mismo estudiar el estado atmosférico en una región como la de la Ciudad de México que en otra del tamaño del país entero pues la percepción de los fenómenos atmosféricos en ambas regiones es bastante diferente. En la región de mayor tamaño puede perderse información que en la pequeña región resulta importante. Es aquí donde es necesario el método de análisis de escala. Este análisis nos permite discernir qué términos de las ecuaciones (2.111) son importantes para describir el estado atmosférico en alguna región de ciertas dimensiones y cuales son despreciables. El principio es simple, sólo debemos cambiar todas las variables por aquellas adimensionales multiplicadas por el correspondiente valor característico.

$$
\begin{array}{cccc}
\bar{x}_{s}=\frac{x_{s}}{L} & \bar{y}_{s}=\frac{y_{s}}{L} & \bar{z}_{s}=\frac{z_{s}}{H_{H}} & \bar{t}=\frac{t}{t_{0}} \\
\bar{r}=\frac{r}{a} & \bar{u}_{s}=\frac{u_{s}}{U_{0}} & \bar{v}_{s}=\frac{v_{s}}{U_{0}} & \bar{w}_{s}=\frac{w_{s}}{W_{0}} \\
\bar{p}=\frac{p}{p_{r}} & \bar{\rho}=\frac{\rho}{\rho_{r}} &
\end{array}
$$

también son de utilidad los siguientes parámetros adimensionales

$$
\begin{aligned}
\epsilon & =\frac{H}{L} \quad \eta=\frac{W_{0}}{U_{0}} & \delta & =\frac{L}{a} \\
\mu & =\frac{U_{0}^{2}}{g L} & t_{0} & =\frac{L}{U_{0}}
\end{aligned} .
$$

Las ecuaciones de momentum adimensionales son

$$
\begin{aligned}
\frac{d \bar{u}_{s}}{d \bar{t}}+\delta \eta \frac{\bar{u}_{s} \bar{w} s}{\bar{r}}-\delta \frac{\bar{u}_{s} \bar{v}_{s}}{\bar{r}} \tan \phi+\frac{2 \Omega L_{0}}{U_{0}}\left(\eta \bar{w}_{s} \cos \phi-\bar{v}_{s} \sin _{I V} \phi\right) & =-\frac{p_{r}}{\rho_{r} U_{0}^{2}} \frac{\cos \phi_{0}}{\bar{r} \cos \phi} \frac{1}{\bar{\rho}} \frac{\partial \bar{p}}{\partial \bar{x}_{s}} \\
\frac{d \bar{v}_{s}}{d \bar{t}}+\delta \eta \frac{\bar{v}_{s} \bar{w}_{s}}{\bar{r}}-\delta \frac{\bar{u}_{s}^{2}}{r} \tan \phi+\frac{2 \Omega L_{0}}{U_{0}} \bar{u}_{s} \sin \phi & =-\frac{p_{r}}{\rho_{r} U_{0}^{2}} \frac{1}{\bar{\rho} \bar{r}} \frac{\partial \bar{p}}{\partial \bar{y}_{s}} \\
\epsilon \eta \frac{d \bar{w}_{s}}{d \bar{t}}-\delta \epsilon \frac{\bar{u}_{s}^{2}+\bar{w}_{s}^{2}}{{ }_{I}}-\frac{2 \Omega L_{0}}{U_{0}} \epsilon \bar{u}_{s} \cos \phi & =-\frac{p_{r}}{\rho_{r} U_{0}^{2}} \frac{1}{\bar{\rho}} \frac{\partial \bar{p}}{\partial \bar{z}_{s}}-\frac{\epsilon}{\mu \bar{r}^{2}} \\
I I I & { }_{I I}
\end{aligned}
$$




\begin{tabular}{|c|c|c|c|c|c|}
\hline \multicolumn{7}{|c|}{ Ecuaciones Horizontales } \\
\hline$I$ & $I I$ & $I I I$ & $I V$ & $V$ & $V I$ \\
\hline $10^{0}$ & $10^{-4}$ & $10^{-1}$ & 10 & $10^{-2}$ & $10^{3}$ \\
\hline \multicolumn{7}{|c|}{ Ecuación Vertical } \\
\hline$I$ & $I I$ & $I I I$ & $I V$ & $V$ & \\
\hline $10^{-5}$ & $10^{-3}$ & $10^{-1}$ & $10^{3}$ & $10^{3}$ & \\
\hline
\end{tabular}

Cuadro 3.1: Valores característicos de las variables atmosféricas y parámetros adimensionales

donde

$$
\frac{d}{d \bar{t}}=\frac{\partial}{\partial \bar{t}}+\frac{\bar{w}_{1} \cos \phi_{0}}{\bar{r} \cos \phi} \frac{\partial}{\partial \bar{x}}+\frac{\bar{w}_{2}}{\bar{r}} \frac{\partial}{\partial \bar{y}}+\frac{\eta}{\epsilon} \bar{w}_{3} \frac{\partial}{\partial \bar{z}}
$$

Con las expresiones dadas en (3.3) y (3.4) podemos cuantificar la importancia relativa de los términos y obtener un modelo adecuado a la escala que queremos. En [10, pág. 6] se muestran los valores característicos de la longitud y el tiempo correspondientes a varios tipos de escalas definidas por varios investigadores a lo largo de varios años.

Como ejemplo usaremos los siguientes valores característicos tomados de [13, pág. 39] para realizar el análisis de escala de las ecuaciones de movimiento

$$
\begin{array}{ccc}
L=10^{3} \mathrm{~km} & U_{0}=10 \mathrm{~ms}^{-1} & W_{0}=10^{-2} \mathrm{~ms}^{-1} \\
\epsilon=10^{-2} & \eta=10^{-3} & \delta=10^{-1} \\
\mu=10^{-5} & t_{0}=L / U=10^{5} \mathrm{~s} & T_{r}=300 \mathrm{~K} \\
\frac{p_{r}}{\rho_{r}}=R T_{r} \simeq 10^{5} \mathrm{~m}^{2} \mathrm{~s}^{-2} & R=287 \mathrm{~J} / \mathrm{kgK}
\end{array} .
$$

Sustituyendo los valores anteriores en (3.3) obtenemos las magnitudes de sus términos los cuales se muestran en la tabla (3.1). Como se puede apreciar los términos de las ecuaciones (3.3) tienen diferentes magnitudes, lo que aprovechamos para su simplificación al eliminar los términos más pequeños. Así las ecuaciones simplificadas de momentum son

$$
\begin{aligned}
\frac{2 \Omega L_{0}}{U_{0}} \bar{v}_{s} \sin \phi & =\frac{p_{r}}{\rho_{r} U_{0}^{2}} \frac{\cos \phi_{0}}{\bar{r} \cos \phi} \frac{1}{\bar{\rho}} \frac{\partial \bar{p}}{\partial \bar{x}_{s}} \\
\frac{2 \Omega L_{0}}{U_{0}} \bar{u}_{s} \cos \phi & =-\frac{p_{r}}{\rho_{r} U_{0}^{2}} \frac{1}{\bar{r} \bar{\rho}} \frac{\partial \bar{p}}{\partial \bar{y}_{s}} \\
\frac{\epsilon}{\mu \bar{r}^{2}} & =-\frac{p_{r}}{\rho_{r}} \frac{1}{\bar{\rho}} \frac{\partial \bar{p}}{\partial \bar{z}_{s}}
\end{aligned}
$$

A las ecuaciones (3.6a) y (3.6b) se les conoce como la aproximación geostrófica y a la ecuación (3.6c) la aproximación hidrostática. La ecuación adimensional de continuidad es, con las magnitudes de cada término

$$
\frac{U_{0}}{L} \frac{d \log \bar{\rho}}{d \bar{t}}+\underbrace{\frac{U_{0}}{L} \frac{1}{\bar{r} \cos \phi}\left(\cos \phi \frac{\partial \bar{u}_{s}}{\partial \bar{x}_{s}}+\frac{\partial \bar{v}_{s} \cos \phi}{\partial \bar{y}_{s}}\right)}_{10^{-5} s^{-1} s^{-1}}+\frac{W_{0}}{H} \frac{\partial \bar{w}_{s}}{\partial \bar{z}_{s}}+2 \frac{W_{0}}{a_{0}} \frac{\bar{w}_{s}}{\bar{r}}=0 .
$$

Por lo que la ecuación simplificada es

$$
\frac{d \log \bar{\rho}}{d \bar{t}}+\frac{1}{\bar{r} \cos \phi}\left(\cos \phi_{0} \frac{\partial \bar{u}_{s}}{\partial \bar{x}_{s}}+\frac{\partial \bar{v}_{s} \cos \phi}{\partial \bar{y}_{s}}\right)+\frac{\eta}{\epsilon} \frac{\partial \bar{w}_{s}}{\partial \bar{z}_{s}}=0
$$

o en forma vectorial

$$
\frac{U_{0}}{L} \frac{d \bar{\rho}}{d \bar{t}}+\bar{\rho} \nabla \cdot \mathbf{W}=0
$$

con

$$
\begin{aligned}
\nabla & =\hat{\lambda} \frac{\cos \phi_{0}}{L \bar{r} \cos \phi} \frac{\partial}{\partial \bar{x}_{s}}+\hat{\phi} \frac{1}{L \bar{r}} \frac{\partial}{\partial \bar{y}_{s}}+\hat{\mathbf{r}} \frac{1}{H} \frac{\partial}{\partial \bar{z}_{s}} \\
\frac{d}{d \bar{t}} & =\frac{\partial}{\partial \bar{t}}+\frac{\bar{u}_{s} \cos \phi_{0}}{\bar{r} \cos \phi} \frac{\partial}{\partial \bar{x}_{s}}+\frac{\bar{v}_{s}}{\bar{r}} \frac{\partial}{\partial \bar{y}_{s}}+\frac{\eta}{\epsilon} \bar{w}_{s} \frac{\partial}{\partial \bar{z}_{s}}
\end{aligned}
$$


entonces

$$
\frac{U_{0}}{L} \frac{d \bar{\rho}}{d \bar{t}}=\frac{U_{0}}{L} \frac{\partial \bar{\rho}}{\partial \bar{t}}+\mathbf{W} \cdot \nabla \bar{\rho}
$$

por lo que la ecuación (3.8) se puede escribir de forma equivalente

$$
\frac{U_{0}}{L} \frac{\partial \bar{\rho}}{\partial \bar{t}}+\underset{10^{-5}}{\nabla \cdot \bar{\rho} \mathbf{W}}=0 .
$$

Dando por resultado la ecuación de continuidad simplificada. Sin embargo, en las ecuaciones de momentum simplificadas (3.6) el término con el operador temporal $d / d t$ se ha despreciado por lo que es razonable suponer que la densidad es independiente del tiempo, por lo que obtenemos la ecuación

$$
\nabla \cdot \bar{\rho} \mathbf{W}=0
$$

que es conocida como la ecuación de continuidad profunda.

\subsection{Estado de referencia atmosférico}

En esta seccion seguimos el procedimineto usado en [23] para probar la existencia de un estado de referencia atmosferico. Las ecuaciones (3.6) y (3.10) son parte del sistema de ecuaciones a resolver para poder determinar de manera única el estado de la atmósfera dentro de los límites definidos en (3.5). Sin embargo, un nuevo problema surge relacionado con la implementación computacional. Resulta que la validez de los incrementos espaciales y temporales $\delta t, \delta x, \delta y$ y $\delta z$ son tales que éstos sean mucho mayores que la distancia entre las moléculas y lo suficientemente pequeñas como para ser considerados constantes [33]. Este criterio nos obliga a considerar incrementos espaciales del orden de centímetros e incrementos temporales de segundos. Si revisamos las cantidades características en (3.5) notaremos que serían necesarios una gran cantidad de datos para poder resolver numéricamente las ecuaciones. Una forma de sortear esta dificultad es promediando las variables termodinámicas sobre una región dada. En la siguiente sección se muestra detalladamente la manera de promediar dichas variables.

\subsubsection{Existencia de un estado de referencia atmosférico}

Es un hecho que la presión atmosférica es una función de las coordenadas $x_{s}, y_{s}, z_{s}$ y del tiempo $t$

$$
p=p\left(x_{s}, y_{s}, z_{s}, t\right)
$$

Ahora supongamos que se puede encontrar alguna parametrización de las coordenadas de la siguiente forma

$$
x_{s}=f_{1}(\xi), y_{s}=f_{2}(\xi), z_{s}=f(\xi) \text {. }
$$

Entonces, la derivada de la presión con respecto al parámetro $\xi$ es

$$
\frac{d p}{d \xi}=\frac{\partial p}{\partial x_{s}} \frac{d f_{1}}{d \xi}+\frac{\partial p}{\partial y_{s}} \frac{d f_{2}}{d \xi}+\frac{\partial p}{\partial z_{s}} \frac{d f}{d \xi} .
$$

Lo que nos interesa es encontrar las superficies de presión constante, entonces la derivada de la presión con respecto al parámetro $\xi$ es cero

$$
\frac{\partial p}{\partial x_{s}} \frac{d f_{1}}{d \xi}+\frac{\partial p}{\partial y_{s}} \frac{d f_{2}}{d \xi}+\frac{\partial p}{\partial z_{s}} \frac{d f}{d \xi}=0
$$

Si proporcionamos explícitamente alguna parametrización de las coordenadas $x_{s}$ y $y_{s}$ entonces la coordenada $z_{s}=f(\xi)$ debe satisfacer la ecuación

$$
\frac{d f}{d \xi}=-\frac{\frac{\partial p}{\partial x_{s}}}{\frac{\partial p}{\partial z_{s}}} \frac{d f_{1}}{d \xi}-\frac{\frac{\partial p}{\partial y_{s}}}{\frac{\partial p}{\partial z_{s}}} \frac{d f_{2}}{d \xi}
$$


Las derivadas $\partial p / \partial x_{s}, \partial p / \partial y_{s}, \partial p / \partial z_{s}$ pueden obtenerse de las ecuaciones de momentum. A la ecuación (3.11) le llamaremos la Ecuación de Isobaras. En forma adimensional la ecuación de las isobaras tiene la forma

$$
\frac{d \bar{f}}{d \bar{\xi}}=-\epsilon\left[\frac{\frac{\partial \bar{p}}{\partial \bar{x}_{s}}}{\frac{\partial \bar{p}}{\partial \bar{z}_{s}}} \frac{d \bar{f}_{1}}{d \bar{\xi}}+\frac{\frac{\partial \bar{p}}{\partial \bar{y}_{s}}}{\frac{\partial \bar{p}}{\partial \bar{z}_{s}}} \frac{d \bar{f}_{2}}{d \bar{\xi}}\right]
$$

Introduzcamos la notación siguiente para el lado izquierdo de las ecuaciones horizontales de momentum

$$
\begin{aligned}
& p_{x} \equiv \frac{d \bar{u}_{s}}{d \bar{t}}-\delta \frac{\bar{u}_{s} \bar{v}_{s}}{\bar{r}} \tan \phi+\delta \eta \frac{\bar{u}_{s} \bar{w}_{s}}{\bar{r}}+\frac{2 \Omega L}{U_{0}} \times\left(\eta \bar{w}_{s} \cos \phi-\bar{v}_{s} \sin \phi\right) \\
& p_{y} \equiv \frac{d \bar{v}_{s}}{d \bar{t}}+\delta \frac{\bar{u}_{s}^{2}}{\bar{r}} \tan \phi+\delta \eta \frac{\bar{v}_{s} \bar{w}_{s}}{\bar{r}}+\frac{2 \Omega L}{U_{0}} \bar{u}_{s} \sin \phi+\frac{\Omega^{2} a L}{U_{0}^{2}} \bar{r} \cos \phi \sin \phi
\end{aligned}
$$

donde usamos los valores característicos para estimar el orden de magnitud de cada término. Para el lado izquierdo de la ecuación de momentum vertical tenemos

$$
\varepsilon \eta \frac{d \bar{w}}{d \bar{t}}-\delta \varepsilon \frac{\bar{u}^{2}+\bar{v}^{2}}{\bar{r}}-\frac{2 \Omega L}{U_{0}} \varepsilon \bar{u} \cos \phi-\frac{\Omega^{2} a L}{U_{0}^{2}} \varepsilon \bar{r} \cos ^{2} \phi+\frac{g H}{U_{0}^{2}} \bar{r}^{-2}=\left(1+\tilde{\mu} p_{z}\right) \frac{\varepsilon}{\tilde{\mu}} \bar{r}^{-2}
$$

donde definimos

$$
p_{z} \equiv\left(\eta \frac{d \bar{w}_{s}}{d \bar{t}}-\delta \frac{\bar{u}_{s}^{2}+\bar{v}_{s}^{2}}{\bar{r}}-\frac{2 \Omega L}{U_{0}} \bar{u}_{s} \cos \phi-\frac{\Omega^{2} a L}{U_{0}^{2}} \bar{r} \cos ^{2} \phi\right) \bar{r}^{22}
$$

Entonces las ecuaciones de momentum toman la forma

$$
\begin{aligned}
p_{x} & =-\frac{p_{r} / \rho_{r}}{U_{0}^{2}} \frac{\cos \phi_{c}}{\cos \phi} \frac{1}{\bar{r} \bar{\rho}} \frac{\partial \bar{p}}{\partial \bar{x}} \\
p_{y} & =-\frac{p_{r} / \rho_{r}}{U_{0}^{2}} \frac{1}{\bar{r}} \frac{\partial \bar{p}}{\partial \bar{y}} \\
\left(1+\tilde{\mu} p_{z}\right) \frac{\varepsilon}{\tilde{\mu}} \bar{r}^{-2} & =-\frac{p_{r} / \rho_{r}}{U_{0}^{2}} \frac{1}{\bar{\rho}} \frac{\partial \bar{p}}{\partial \bar{z}}
\end{aligned}
$$

de la cual obtenemos los cocientes

$$
\frac{\frac{\cos \phi_{c}}{\cos \phi} \frac{1}{\bar{r}} \frac{\partial \bar{p}}{\partial \bar{x}}}{\frac{\partial \bar{p}}{\partial \bar{z}}}=\frac{\tilde{\mu}}{\varepsilon} \bar{r}^{2} \frac{p_{x}}{1+\tilde{\mu} p_{z}} \quad \frac{\frac{1}{\bar{r}} \frac{\partial \bar{p}}{\partial \bar{y}}}{\frac{\partial \bar{p}}{\partial \bar{z}}}=\frac{\tilde{\mu}}{\varepsilon} \bar{r}^{2} \frac{p_{y}}{1+\tilde{\mu} p_{z}}
$$

y la ecuación de isobaras queda como sigue

$$
\frac{d \bar{f}_{s}}{d \bar{\xi}_{s}}=-\frac{\tilde{\mu}}{\varepsilon}\left[\cos \theta \frac{\cos \phi}{\cos \phi_{c}} p_{x}+\sin \theta p_{y}\right] \frac{\bar{r}^{3}}{1+\tilde{\mu} p_{z}} .
$$

El parámetro relevante para estimar el orden de magnitud del lado derecho es

$$
\mu=\frac{\tilde{\mu}}{\varepsilon}
$$

y si usamos los valores característicos anteriores

$$
\begin{aligned}
\mu & =\frac{\tilde{\mu}}{\varepsilon}=\frac{U_{0}^{2}}{g H}=\frac{10^{2} \mathrm{~m}^{-2} \mathrm{~s}^{-2}}{10 \mathrm{~m}^{-1} \mathrm{~s}^{-2} \times 10^{4} \mathrm{~m}^{-2}}=10^{-3} \\
\tilde{\mu} & =\mu \varepsilon=10^{-5} \\
\bar{r} & \sim 1
\end{aligned}
$$


obtenemos

$$
\frac{d \bar{f}_{s}}{d \bar{\xi}_{s}}=-\mu \quad\left[\cos \theta \frac{\cos \phi}{\cos \phi_{c}} p_{x}+\sin \theta p_{y}\right] \frac{\bar{r}^{3}}{1+\mu \varepsilon p_{z}}
$$

De acuerdo con lo anterior la ecuación (3.14) tiene la forma

$$
\frac{d \bar{f}_{s}}{d \bar{\xi}_{s}}=\mu F\left(\bar{\xi}_{s}, \bar{f}_{s}, \bar{t}, \theta_{s}, \mu\right) \quad \text { con } \bar{f}_{s}=\bar{z}_{0} \quad \text { en } \bar{\xi}_{s}=0 .
$$

donde $\mu=10^{-3}$ es un parámetro pequeño y $F \sim 10^{2}$ lo que justifica la aplicación del siguiente resultado.

Teorema 3.1 [12, p 213] Sea $\mathbf{X}=\left(x_{1}, x_{2}, \ldots, x_{n}\right)^{t}$ un vector columna con $n$ componentes que dependen de un parámetro $\xi$. Si $\mathbf{F}(\mathbf{X}, \xi, \mu)$ es analítica en $x_{1}, x_{2}, \ldots, x_{n}, \mu, y$ continua en $\xi$, entonces el sistema de ecuaciones diferenciales ordinarias

$$
\frac{d \mathbf{X}}{d \xi}=\mathbf{F}(\mathbf{X}, \xi, \mu)
$$

bajo la condición $\mathbf{X}(\xi=0, \mu)=0$, tiene solución única con la forma

$$
\mathbf{X}=\sum_{k=0}^{\infty} \mathbf{X}^{(k)}(\xi) \mu^{k}
$$

donde $\mathbf{X}^{(k)}(\xi)$ es una función vectorial de $\xi$.

En nuestro caso el lado derecho de (3.14) es una función analítica de $\mu$ en una vecindad de $\mu=0$ y podemos suponer que $F\left(\bar{\xi}_{s}, \bar{f}_{s}, \bar{t}, \theta_{s}, \mu\right)$ es analítica en $\bar{f}_{s}$ y continua en $\bar{\xi}_{s}$. Entonces el teorema anterior afirma que existe una solución de la forma

$$
\bar{f}_{s}=\sum_{k=0}^{\infty} f^{(k)}\left(\bar{\xi}_{s}, \bar{f}_{s}, \bar{t}, \theta_{s}\right) \mu^{k}
$$

Es fácil de ver que los coeficientes $f_{s}^{(k)}$ satisfacen las condiciones de frontera

$$
\bar{f}^{(0)}=\bar{z}_{0}, \quad \bar{f}^{(k)}=0 \quad \text { para } k \geq 0
$$

Reemplazando la serie (3.18) en (3.15) obtenemos la solución a orden cero.

$$
f^{(0)}\left(\xi_{s}, t, \theta_{s}\right)=z_{0}
$$

Esto significa que a orden cero la isobara que pasa a través de $\left(x_{s}=y_{s}=0, z_{s}=z_{s 0}\right)$ depende únicamente de $z_{s}$ y, en consecuencia, el campo de presión tiene la forma

$$
p(\mathbf{r}, t)=p^{(0)}\left(z_{s}, t\right)+\sum_{k=0}^{\infty} p^{(k)}(\mathbf{r}, t) \mu^{k}
$$

Este resultado riguroso es sobresaliente ya que es independiente de la distribución de temperatura $T$ (r,t) $y$ densidad $\rho(\mathbf{r}, t)$.

Para la temperatura y la densidad proponemos los desarrollos

$$
T=\sum_{k=0}^{\infty} T^{(k)} \mu^{k} \quad \rho=\sum_{k=0}^{\infty} \rho^{(k)} \mu^{k} .
$$

Sustituyendo los desarrollos (3.19) y (3.20) en la ecuación de estado

$$
p(\mathbf{r}, T)=R T(\mathbf{r}, T) \rho(\mathbf{r}, T)
$$

obtenemos

$$
\sum_{k=0}^{\infty} \mu^{k} p^{(k)}=R \sum_{k=0}^{\infty} \mu^{k}\left[\sum_{m=0}^{\infty} T^{(m)} \rho^{(k-m)}\right]
$$


donde usamos

$$
T \rho=\left(\sum_{m=0}^{\infty} \mu^{m} T^{(m)}\right)\left(\sum_{l=0}^{\infty} \mu^{l} \rho^{(l)}\right)=\sum_{k=0} \mu^{k}\left(\sum_{m=0}^{k} T^{(m)} \rho^{(k-l)}\right) .
$$

Por tanto

$$
p^{(k)}=\mathcal{R} \sum_{m=0}^{k} T^{(m)} \rho^{(k-m)} \quad \text { para } k \geq 0
$$

A orden cero tenemos

$$
p^{(0)}\left(z_{s}, t\right)=R T^{(0)}(\mathbf{r}, t) \rho^{(0)}(\mathbf{r}, t)
$$

de donde es natural suponer que el lado derecho es sólo función de $z_{s}$ y $t$,es decir

$$
T^{(0)}=T^{(0)}\left(z_{s}, t\right) \quad \rho^{(0)}=\rho^{(0)}\left(z_{s}, t\right) .
$$

De acuerdo con lo anterior: Cada variable termodinámica admite un desarrollo en potencias del parámetro $\mu$ con la forma

$$
\psi(\mathbf{r}, t)=\psi^{(0)}\left(z_{s}, t\right)+\sum_{k=1}^{\infty} \psi^{(k)}(\mathbf{r}, t) \mu^{k} .
$$

Los términos de orden cero $p^{(0)}\left(z_{s}, t\right), \rho^{(0)}\left(z_{s}, t\right)$ y $T^{(0)}\left(z_{s}, t\right)$ satisfacen la ecuación de estado

$$
p^{(0)}\left(z_{s}, t\right)=\mathcal{R} T^{(0)}\left(z_{s}, t\right) \rho^{(0)}\left(z_{s}, t\right)
$$

y definen lo que en lo que podemos llamar estado de referencia termodinámico de la atmósfera o, simplemente, estado de referencia atmosférico.

Para simplificar la notación podemos expresar cada desarrollo (3.21) como sigue

$$
\psi^{(0)}\left(z_{s}, t\right) \equiv \psi_{0}\left(z_{s}, t\right) \quad \sum_{k=1}^{\infty} \psi^{(k)}(\mathbf{r}, t) \mu^{k} \equiv \psi_{1}(\mathbf{r}, t)
$$

con lo cual tenemos

$$
\psi(r, t)=\psi_{0}\left(z_{s}, t\right)+\psi_{1}(\mathbf{r}, t)
$$

$\mathrm{Y}$ para $p, \rho$ y $T$ tenemos

$$
\begin{gathered}
p(\mathbf{r}, t)=p_{0}\left(z_{s}, t\right)+p_{1}(\mathbf{r}, t)=p_{0}+p_{1} \\
\rho(\mathbf{r}, t)=\rho_{0}\left(z_{s}, t\right)+\rho_{1}(\mathbf{r}, t)=\rho_{0}+\rho_{1} \\
T(\mathbf{r}, t)=T_{0}\left(z_{s}, t\right)+T_{1}(\mathbf{r}, t)=T_{0}+T_{1} .
\end{gathered}
$$

El resultado primario que hemos demostrado es la existencia del desarrollo (3.18) para la presión y hemos supuesto que existen desarrollos similares (3.19) para $\rho$ y $T$. Hay dos casos de interés donde los desarrollos (3.19) son obvios para $\rho$ y $T$ :

1. Atmósfera isotérmica. En este caso es obvio la validez del desarrollo para $\rho$ ya que, de acuerdo con la ecuación de estado, $\rho$ es una función lineal de la presión y por tanto

$$
\rho=\frac{p(\mathbf{r}, t)}{\mathcal{R} T}=\frac{p^{(0)}\left(z_{s}, t\right)}{\mathcal{R} T}+\sum_{k=0}^{\infty} \frac{p^{(k)}(\mathbf{r}, t)}{\mathcal{R} T} \mu^{k} .
$$

2. Atmósfera isoentrópica. De acuerdo con las fórmulas de Poisson

$$
T=T_{0}\left(\frac{p}{\rho_{0}}\right)^{\frac{R}{c_{p}}} \quad \rho=\rho_{0}\left(\frac{p}{p_{0}}\right)^{\frac{c_{v}}{c_{p}}} .
$$

es obvio que $\rho$ y $T$ tiene desarrollos en $\mu$ ya que son funciones analíticas de la presión $p(\mathbf{r}, t)$. 
Nota 3.1 . Es interesante observar que el uso de la aproximación hidrostática

$$
-\frac{1}{\rho} \frac{\partial p}{\partial z_{s}}=g \frac{a^{2}}{r^{2}}+A_{z} \sim g \text {. }
$$

constituye una perturbación regular de la ecuación de isobaras.

En efecto, la ecuación de momentum vertical adimensionalizada es

$$
-\frac{p_{r} / \rho_{r}}{U_{0}^{2}} \frac{1}{\bar{\rho}} \frac{\partial \bar{p}}{\partial \bar{z}}=\left(1+\tilde{\mu} p_{z}\right) \frac{1}{\mu} \bar{r}^{-2}
$$

y al calcular los cocientes (3.13) podemos desarrollar en forma regular el cociente

$$
\frac{1}{1+\mu \varepsilon p_{z}}=\left(1+\mu \varepsilon p_{z}\right)^{-1}=1-\underbrace{\mu \varepsilon p_{z}}_{10^{-3}}+O\left(10^{-6}\right) \sim 1
$$

lo que equivale exactamente a usar la aproximación hidrostática!

$$
-\frac{1}{\rho} \frac{\partial p}{\partial z_{s}} \sim g
$$

por lo que tal aproximación no implica una pérdida significativa de información o precisión en el cálculo de isobaras y en la definición del estado de referencia atmosférico.

\subsubsection{Estimación del valor de referencia $\psi^{(0)}\left(z_{s}, t\right)$ con un flujo bidimensional.}

En esta sección consideramos el cálculo de $\psi^{(0)}\left(z_{s}, t\right)$ en $(3.22)$ por medio de el promedio espacial

$$
\hat{\psi}\left(z_{s}\right)=\frac{1}{L_{s}} \int_{-L_{s} / 2}^{+L_{s} / 2} \psi\left(x_{s}, z_{s}\right) d x_{s}
$$

para el caso de un flujo bidimensional estacionario en el plano $x z$. El flujo en cuestión $\mathbf{v}=u \mathbf{i}+w \mathbf{k}$ es una solución analítica de la ecuación [33] de continuidad somera

$$
\nabla \cdot \mathbf{v}(\mathbf{r})=0
$$

con la condición de frontera

$$
\mathbf{v} \cdot \mathbf{n}=0 \quad \text { sobre } z=h(x)
$$

donde $h(x)$ es la elevación del terreno en el punto $(x, y=0, z=0)$. En la figura 1 se muestra la región y algunos puntos donde $\mathbf{v}$ es calculado. Los datos usados para calcular $h(x)$ por medio de polinomios cúbicos provienen de la base de datos GTOPO30 [41]. El campo $\mathbf{v}=u \mathbf{i}+w \mathbf{k}$ es calculado con el dato $u=10 \mathrm{~ms}^{-1}$ y $w=0$ en el punto $(x=0, z=10 \mathrm{~km})$.

\subsubsection{Estimación de $p^{(0)}\left(z_{s}, t\right)$}

Consideremos el cálculo del término $p^{(0)}\left(z_{s}, t\right)$ correspondiente a la descomposición

$$
p\left(x_{s}, z_{s}\right)=p^{(0)}\left(z_{s}\right)+\sum_{k=1}^{\infty} p^{(k)}(\mathbf{r}) \mu^{k},
$$

para la presión $p$, usando el promedio espacial

$$
\hat{p}\left(z_{s}\right)=\frac{1}{L_{s}} \int_{-L_{s} / 2}^{+L_{s} / 2} p\left(x_{s}, z_{s}\right) d x_{s}
$$




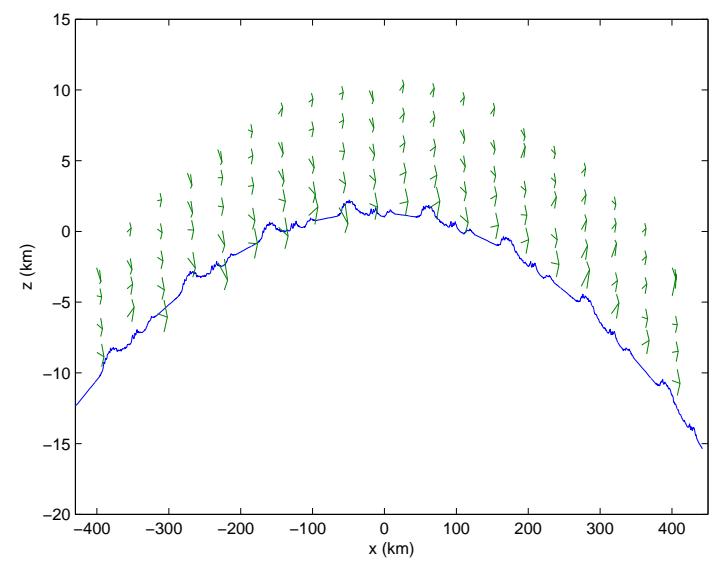

Figura 3.1: Topografía y campo de velocidad V

Las ecuaciones de Bernoulli son usadas para obtener el campo de presión en el punto $\mathbf{r}=(x, z)$,

$$
\frac{p(\mathbf{r})}{\rho_{0}}=C_{0}-\frac{1}{2} v^{2}(\mathbf{r})+\phi_{g}(\mathbf{r})
$$

donde $v^{2}=u^{2}+w^{2}, \rho_{0}=1 \mathrm{~kg} / \mathrm{m}^{3}, \phi_{g}(\mathbf{r})=-g a^{2} /\left(z_{s}+a\right)$ es el potencial gravitacional, $a=6376,98 \mathrm{~km}$ es el radio de la Tierra y $C_{0}=-62428 \times 10^{4} \mathrm{~m}^{2} / \mathrm{s}^{2}$ es calculado con $p(\mathbf{r})=324,84 \mathrm{mb}, u=10,0 \mathrm{~m} / \mathrm{s}, w=0,13$ $\mathrm{m} / \mathrm{s} \phi_{g}(\mathbf{r})=-62460 \times 10^{4} \mathrm{~m}^{2} / \mathrm{s}^{2}$ en $\left(x=0, y=0, z_{s}=10 \mathrm{~km}\right)$. El error relativo de $\hat{p}\left(z_{s}\right)$ con respecto a el valor exacto $p\left(x, z_{s}\right)$,

$$
\Delta \hat{p}\left(z_{s}\right)=\left(1-\frac{\hat{p}\left(z_{s}\right)}{p\left(x_{s}, z_{s}\right)}\right) \times 100
$$

es una medida correcta de la exactitud de $\hat{p}$ si ésta es vista como una aproximación de $p\left(x_{s}, z_{s}\right)$ y el estado de referencia $p^{(0)}\left(z_{s}\right)$.

El cuadro 3.2 muestra los resultados para la presión. Sorprendentemente, vemos que $\hat{p}\left(z_{s}\right)$ tiene un error relativo $\left|\Delta \hat{p}\left(z_{s}\right)\right|$ que es menor a $0,1 \%$ para $L_{s}$ de 50 a $800 \mathrm{~km}$, así que para propósitos prácticos $\hat{p}\left(z_{s}\right)$ es casi igual a $p\left(x, z_{s}\right) \mathrm{y}$, en consecuencia, el campo $p(\mathbf{r})$ tiene la descomposición

$$
p\left(x_{s}, z_{s}\right)=\hat{p}\left(z_{s}\right)+\delta \hat{p}\left(x_{s}, z_{s}\right)
$$

con $|\delta \hat{p} / p|<10^{-1} \%$. En [24] se reportan soluciones analíticas de la ecuación de continuidad profunda $\nabla \cdot \rho_{0}(\mathbf{r}) \mathbf{U}(\mathbf{r})=0$. El uso del campo $\mathbf{U}$ nos deja, básicamente, los mismos resultados del cuadro (3.2). Estos resultados justifican la descomposición (3.28) y la estimación de $\psi^{(0)}\left(z_{s}, t\right)$ por medio de un promedio espacial $\hat{\psi}\left(z_{s}\right)(3.25)$.

\begin{tabular}{|c|cc|cc|cc|}
\hline$L_{s}$ & \multicolumn{2}{|c|}{$\min \{\Delta \hat{p}\}$} & \multicolumn{2}{c|}{$\max \{\Delta \hat{p}\}$} & \multicolumn{2}{c|}{$\hat{p}$} \\
\hline & $2 \mathrm{~km}$ & $10 \mathrm{~km}$ & $2 \mathrm{~km}$ & $10 \mathrm{~km}$ & $2 \mathrm{~km}$ & $10 \mathrm{~km}$ \\
$800 \mathrm{~km}$ & -0.1 & 0.0 & 0.1 & 0.0 & 1106.64 & 324.85 \\
$400 \mathrm{~km}$ & -0.1 & 0.0 & 0.1 & 0.0 & 1106.60 & 324.85 \\
$100 \mathrm{~km}$ & -0.1 & 0.0 & 0.0 & 0.0 & 1106.65 & 324.85 \\
$50 \mathrm{~km}$ & 0.0 & 0.0 & 0.0 & 0.0 & 1106.67 & 324.86 \\
\hline
\end{tabular}

Cuadro 3.2: Valores de $\check{p}, \min \{\Delta \check{p}\}$ y $\max \{\Delta \breve{p}\}$ para la presión (en mb) a $z=2,10 \mathrm{~km}$. usando la descomposición (3.28)

\subsubsection{Región de validez de la descomposición estándar $\psi(t, \mathbf{r})=\psi_{0}(z)+\bar{\psi}(t, \mathbf{r})$}

Consideremos la descomposición usual en mesoescala 


$$
\psi(t, \mathbf{r})=\psi_{0}(z)+\bar{\psi}(t, \mathbf{r})
$$

donde los valores de referencia $\psi_{0}(z)$ son estimados por medio del promedio espacial

$$
\breve{\psi}(z)=\frac{1}{L} \int_{-L / 2}^{+L / 2} \psi(x, z) d x .
$$

El error relativo de $\breve{\psi}(z)$ con respecto a el valor exacto $\psi(x, z)$,

$$
\Delta \breve{\psi}(z)=\left(1-\frac{\breve{\psi}(z)}{\psi(x, z)}\right) \times 100
$$

es una medida de la exactitud de $\breve{\psi}$ si es vista como una aproximación de $\psi(x, z)$ y del estado de referencia. El cuadro (3.3) reporta los resultados para la presión obtenidos del mismo flujo que en el cuadro (3.3) y de la ecuación de Bernoulli. Ahí vemos que el error relativo $|\Delta \check{p}|$ es menor que $5 \%$ para $L \leq 100 \mathrm{~km}$. Del uso de soluciones analíticas de la ecuación de continuidad profunda $\nabla \cdot \rho_{0}(\mathbf{r}) \mathbf{U}(\mathbf{r})=0$ se obtienen, básicamente, los mismos valores de el cuadro (3.3). Si consideramos que el promedio de $\check{p}(z)$ es obtenido de un campo exacto $p(x, z)$ mientras que en situaciones reales $\check{p}(z)$ es estimado de datos de una red de monitoreo y, por lo tanto, su precisión con respecto a los valores verdaderos de $\check{p}(z)$, puede ser pobre por lo que la validez de la descomposición (3.30) puede ser significativamente menor que $100 \times 100 \mathrm{~km}^{2}$.

\begin{tabular}{|c|cc|cc|cc|}
\hline$L_{s}$ & \multicolumn{2}{|c|}{$\min \{\Delta \hat{p}\}$} & \multicolumn{2}{c|}{$\max \{\Delta \hat{p}\}$} & \multicolumn{2}{c|}{$\hat{p}$} \\
\hline & $2 \mathrm{~km}$ & $10 \mathrm{~km}$ & $2 \mathrm{~km}$ & $10 \mathrm{~km}$ & $2 \mathrm{~km}$ & $10 \mathrm{~km}$ \\
$400 \mathrm{~km}$ & -20.2 & -91.5 & 10.4 & 45.8 & 1102.63 & 222.82 \\
$200 \mathrm{~km}$ & -4.7 & -17.0 & 2.4 & 8.5 & 1080.71 & 299.28 \\
$100 \mathrm{~km}$ & -2.6 & -9.2 & 1.3 & 4.6 & 1092.42 & 310.44 \\
$150 \mathrm{~km}$ & -1.2 & -4.0 & 0.5 & 2.0 & 1100.87 & 318.42 \\
$50 \mathrm{~km}$ & -0.3 & -1.0 & 0.2 & 0.5 & 1105.02 & 323.20 \\
\hline
\end{tabular}

Cuadro 3.3: Valores de $\check{p}, \min \{\Delta \check{p}\}$ y $\max \{\Delta \check{p}\}$ para la presión (en mb) a $z=2,10 \mathrm{~km}$. usando la descomposición (3.30)

\subsubsection{Estimación del orden de magnitud del cociente $\psi_{1}(\mathbf{r}, t) / \psi_{0}(\mathbf{r}, t)$}

El campo de velocidad $\mathbf{v}$ alrededor de la esfera y elipsoide pueden usarse para "corroborar" el análisis de escalas.

Usando la ecuación de Bernoulli obtenemos una expresión analítica de la presión a partir de un campo de velocidad v Por ejemplo, usando

$$
\frac{p(\mathbf{r})}{\rho_{0}}=c_{0}-\frac{1}{2} v^{2}(\mathbf{r})+\phi_{g}(\mathbf{r})
$$

podemos proponer la estimación del término de referencia $p^{(0)}\left(z_{s}\right)$ por medio de un promedio espacial como:

$$
\widehat{p}\left(z_{s}\right)=\frac{1}{S} \iint_{S} d s p(\mathbf{r})
$$

y comparar $\widehat{p}\left(z_{s}\right)$ contra $p(\mathbf{r})$ sobre superficies $z_{s}=$ cte. Un método simple para estimar $\psi_{1} / \psi_{0}$ es usar los resultados de la sección anterior.

\subsubsection{Resultados con el flujo v sobre una topografía bidimensional}

El promedio espacial

$$
\widehat{p}_{0}\left(z_{s}\right)=\frac{1}{L_{s}} \int_{-L_{s} / 2}^{L_{s} / 2} p\left(x_{s}, z_{s}\right) d x_{s}
$$


tiene un error relativo

$$
\left|\frac{\delta p}{p}\right|=\left|\frac{\widehat{p}_{0}\left(z_{s}\right)-p\left(x_{s}, z_{s}\right)}{p\left(x_{s}, z_{s}\right)}\right|
$$

acotado por $10^{-3}$ para $z_{s}=2$ y $10 \mathrm{~km} \mathrm{y}$

$$
\left|\frac{\delta p}{p}\right| \leq 10^{-3} \quad \text { con } 50 \mathrm{~km} \leq L_{s} \leq 800 \mathrm{~km}
$$

ó

$$
|\delta p| \leq 10^{-3} p \quad \text { con } 50 \mathrm{~km} \leq L_{s} \leq 800 \mathrm{~km}
$$

En esta forma podemos proponer la descomposición

$$
p(\mathbf{r})=\widehat{p}_{0}\left(z_{s}\right)+\delta p
$$

donde la corrección $\delta p$ satisface

$$
\left|\frac{\delta p}{p}\right|<10^{-3}
$$

ó, equivalentemente,

$$
\begin{aligned}
p & \sim p_{0} \\
\delta p & \sim 10^{-3} p_{0} \quad \text { ó } \delta p \sim 10^{-3} p .
\end{aligned}
$$

En resumen, la presión $p(\mathbf{r}, t)$ admite la descomposición

$$
p=p_{0}\left(z_{s}, t\right)+p_{1}(\mathbf{r}, t)
$$

donde

$$
p_{1} \lesssim 10^{-3} p_{0} \quad \text { para } 10^{2} \mathrm{~km} \leq L_{s} \leq 10^{3} \mathrm{~km} .
$$

Para $\rho(\mathbf{r}, t)$ y $T(\mathbf{r}, t)$ proponemos

$$
\begin{aligned}
\rho & =\rho_{0}\left(z_{s}, t\right)+\rho_{1}(\mathbf{r}, t) \\
T & =T_{0}\left(z_{s}, t\right)+T_{1}(\mathbf{r}, t)
\end{aligned}
$$

donde $\rho$ y $T$ y los términos de referencia $\rho_{0}$ y $T_{0}$ satisfacen la ecuación de estado

$$
\begin{aligned}
p_{0} & =R T_{0} \rho_{0} \\
p & =R T \rho .
\end{aligned}
$$

Sustituyendo (3.37)-(3.39) en (3.41) y usando (3.40) se obtiene

$$
p_{0}+p_{1}=\mathcal{R}\left(T_{0}+T_{1}\right)\left(\rho_{0}+\rho_{1}\right)=\mathcal{R}\left(T_{0} \rho_{0}\right)+\mathcal{R}\left(T_{1} \rho_{0}+T_{0} \rho_{1}\right)
$$

que se reduce a

$$
p_{1}=\mathcal{R}\left(T_{1} \rho_{0}+T_{0} \rho_{1}\right)
$$

y dividiendo miembro a miembro con (3.41),

$$
\frac{p_{1}}{p_{0}}=\frac{\mathcal{R}\left(T_{1} \rho_{0}+T_{0} \rho_{1}\right)}{\mathcal{R} T_{0} \rho_{0}}=\frac{T_{1}}{T_{0}}+\frac{\rho_{1}}{\rho_{0}},
$$

se llega a

De acuerdo con relación

$$
\frac{p_{1}}{p_{0}}=\frac{T_{1}}{T_{0}}+\frac{\rho_{1}}{\rho_{0}} .
$$

$$
\left|\frac{p_{1}}{p_{0}}\right| \lesssim 10^{-3}
$$


se obtiene

$$
\left|\frac{T_{1}}{T_{0}}+\frac{\rho_{1}}{\rho_{0}}\right| \lesssim 10^{-3}
$$

Los valores de referencia $T_{0}$ y $\rho_{0}$ son positivos y las perturbaciones $T_{1}$ y $\rho_{1}$ pueden cambiar de signo. En el caso particular de que la temperatura $T=T_{0}+T_{1}$ sea ligeramente mayor a $T_{0}$ tenemos

$$
0<\frac{T_{1}}{T_{0}}+\frac{\rho_{1}}{\rho_{0}} \lesssim 10^{-3}
$$

por lo tanto

$$
\begin{aligned}
T_{1} & \lesssim 10^{-3} T_{0} \\
\rho_{1} & \lesssim 10^{-3} \rho_{0}
\end{aligned}
$$

lo que podemos extender al caso en que las perturbaciones $T_{1}$ y $\rho_{1}$ sean negativas

$$
\begin{aligned}
\left|T_{1}\right| \lesssim 10^{-3} T_{0} \\
\left|\rho_{1}\right| \lesssim 10^{-3} \rho_{0},
\end{aligned}
$$

dado que su magnitud es acotada, para una escala horizontal $L_{s}$ en el rango siguiente

$$
10^{2} \mathrm{~km} \leq L_{s} \leq 10^{3} \mathrm{~km} .
$$

\subsection{Simplificación de la ecuación para $w_{s}$}

En esta sección usaremos las estimaciones del estado de referencia y valores característicos con una escala horizontal aproximada de $L \sim 10^{3} \mathrm{~km}$ para simplificar las ecuaciones de movimiento vertical. Dicha ecuación para una Tierra esférica es

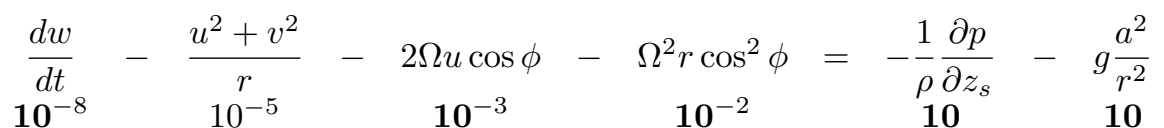

Estimación del orden de magnitud de los términos con los valores característicos

$$
U \sim 10 \mathrm{~ms}^{-1} \quad W \sim 10^{-2} \mathrm{~ms}^{-1} \quad L \sim 10^{6} \mathrm{~m}
$$

y la escala de tiempo $t_{c}$ la estimamos a partir de $U=L / t_{c}$, de donde se obtiene

$$
t_{c} \sim \frac{L}{U} \quad \text { ò } \quad \frac{1}{t_{c}}=\frac{U}{L}=10^{-5} \mathrm{~s}^{-1} .
$$

entonces

$$
\begin{aligned}
& \frac{d w_{s}}{d t} \sim W / t_{c}=10^{-2} \mathrm{~ms}^{-1} \times 10^{-5} \mathrm{~s}^{-1} \quad=\quad 10^{-7} \mathrm{~ms}^{-2} \\
& \frac{u_{s}^{2}+v_{s}^{2}}{r} \sim \frac{U^{2}}{a}=\frac{10^{2} \mathrm{~m}^{2} \mathrm{~s}^{-2}}{6,378 \times 10^{6} \mathrm{~m}}=1,6 \times 10^{-5} \mathrm{~ms}^{-2} \\
& 2 \Omega u_{s} \sim 2 \Omega U=2 \times 7 \times 10^{-5} \times 10 \mathrm{~ms}^{-2}=1,4 \times 10^{-3} \mathrm{~ms}^{-2} \\
& \Omega^{2} r \sim \Omega^{2} a=49 \times 10^{-10} \mathrm{~s}^{-2} \times 6,3 \times 10^{6} \mathrm{~m}=3,1 \times 10^{-2} \mathrm{~ms}^{-2} \\
& g \sim \quad=\quad 10 \mathrm{~ms}^{-2}
\end{aligned}
$$

a) El término dominante en el lado izquierdo de (3.43) corresponde a la aceleración centrípeta

$$
\Omega^{2} a \bar{r} \cos ^{2} \phi \sim 3 \times 10^{-2} \mathrm{~ms}^{-2} .
$$

b) El término dominante en el lado derecho de (3.43) es

$$
g \sim 10 \mathrm{~ms}^{-2}
$$


por lo tanto, necesariamente debemos tener

$$
\frac{-1}{\rho} \frac{\partial p}{\partial z_{s}} \sim 10 \mathrm{~ms}^{-2}
$$

Esto es correcto ya que

$$
\begin{aligned}
p^{0} & \sim 10^{5} \mathrm{~Pa}=10^{5} \mathrm{Nm}^{-2} \\
H & \sim 10^{4} \mathrm{~m} \\
\rho^{0} & \sim 1 \mathrm{~kg} \mathrm{~m}{ }^{-3}
\end{aligned}
$$

de manera que

$$
\frac{-1}{\rho} \frac{\partial p}{\partial z_{s}} \sim \frac{p^{0}}{\rho^{0} H}=\frac{10^{5} \mathrm{Nm}^{-2}}{10^{4} \mathrm{~kg} \mathrm{~m}^{-3} \mathrm{~m}}=10 \mathrm{~ms}^{-2}
$$

Por tanto la ecuación de momentum se reduce a la bien conocida aproximación hidrostática

$$
\frac{1}{\rho} \frac{\partial p}{\partial z_{s}}=-g \frac{a^{2}}{r^{2}}
$$

De acuerdo con (3.37) y (3.38) podemos aproximar $\rho$ y por los valores de referencia $\rho_{0}$ y $p_{0}$, esto es

$$
\frac{1}{\rho_{0}} \frac{\partial p_{0}}{\partial z_{s}}=-g \frac{a^{2}}{r^{2}}
$$

lo cual es consistente con el hecho de que $\rho_{0}$ y $p_{0}$ son funciones de $z_{s}$

$$
p_{0}=p_{0}\left(z_{s}, t\right), \quad \rho_{0}=\rho_{0}\left(z_{s}, t\right)
$$

y el lado derecho de (3.45) también lo es. Pero debemos señalar lo siguiente: La ecuación (3.45) está en forma Euleriana

$$
\frac{1}{\rho_{0}\left(z_{s}, t\right)} \frac{\partial p_{0}\left(z_{s}, t\right)}{\partial z_{s}}=-g \frac{a^{2}}{\left(z_{s}+a\right)^{2}}
$$

y dado que el lado derecho no depende explícitamente del tiempo $t$, entonces el lado izquierdo tampoco lo hace, por lo que podemos proponer

$$
\rho_{0}=\rho_{0}\left(z_{s}\right) \quad p_{0}=p_{0}\left(z_{s}\right)
$$

Esto reduce la ecuación de continuidad

$$
\frac{d \rho_{0}}{d t}+\rho_{0} \nabla \cdot \mathbf{v}=0
$$

a la forma

$$
\nabla \cdot \rho_{0}\left(z_{s}\right) \mathbf{v}=0
$$

conocida como ecuación de continuidad profunda.

\subsection{Algunos estados de referencia hidrostáticos}

En la literatura estándar y en modelos computacionales se acostumbra estimar el estado de referencia atmosférico con los valores de $p, \rho, T$ correspondientes a una atmósfera hidrostática sobre un modelo esférico terrestre, despreciando el efecto de la aceleración centrípeta, lo que equivale a hacer $\mathbf{a}=\boldsymbol{\Omega}=\mathbf{0}$ en la ecuación de momentum

$$
\mathbf{a}+2 \boldsymbol{\Omega} \times \mathbf{v}+\boldsymbol{\Omega} \times(\boldsymbol{\Omega} \times \mathbf{R})=-\frac{1}{\rho} \nabla p+\mathbf{g}
$$

Si, adicionalmente, consideramos que la atmósfera es barotrópica tenemos $\rho=\rho(p)$ con lo que calculamos la función

$$
P[p] \equiv \int^{p} \frac{d s}{\rho(s)}
$$


en términos de la cual la ecuación de momentum se reduce a

$$
\nabla\left[P-\Phi_{g}\right]=\mathbf{0}
$$

donde sólo aparece el potencial gravitacional de una esfera con radio a

$$
\Phi_{g}=g \frac{a^{2}}{r}
$$

De acuerdo con lo anterior las superficies con $P=$ constante coinciden con las superficies equipotenciales del campo de gravedad,

$$
P\left(p_{0}\right)-\Phi_{g}\left(z_{s}\right)=C
$$

donde agregamos el subíndice o para indicar valores de referencia. La constante de integración $C$ se determina con los valores de referencia de la presión y la temperatura en la superficie terrestre $p_{00}=10^{5} \mathrm{~Pa}, T_{00}=$ $300 \mathrm{~K}$,

$$
C=P\left(p_{00}\right)-\Phi_{g}\left(z_{s}=0\right)=P\left[p_{00}\right]-g a .
$$

En esta forma obtenemos $P(p)-\Phi_{g}\left(z_{s}\right)=P\left[p_{00}\right]-g a$ ó

$$
P\left[p_{0}\right]-P\left[p_{00}\right]=\Phi_{g}\left(z_{s}\right)-g a=-g a\left(1-\frac{a}{r}\right)
$$

donde $r=z_{s}+a$. Como casos particulares tenemos.

\subsubsection{Atmósfera isotérmica}

En el caso de una atmósfera isotérmica tenemos

$$
P\left[p_{0}\right]-P\left[p_{00}\right]=R T \ln \frac{p_{0}}{p_{00}}=-g a\left(1-\frac{a}{r}\right),
$$

por lo tanto

donde definimos

$$
p_{0}\left(z_{s}\right)=p_{00} \exp \left[-\frac{a}{H}\left(1-\frac{a}{r}\right)\right]
$$

$$
H \equiv \frac{R T_{00}}{g}
$$

como la altura característica de una atmósfera isotérmica. Con los valores estándar $R=2875 \mathrm{~J} / \mathrm{kgK}, g=9,8$ $\mathrm{ms}^{-2}, T_{00}=300 \mathrm{~K}$ tenemos

$$
H=8,78 \mathrm{~km} \text {. }
$$

\subsubsection{Atmósfera adiabática}

Para una atmósfera adiabática tenemos la fórmula de Poisson

$$
\rho_{0}^{-1}=\rho_{* 0}^{-1}\left(\frac{p_{0}}{p_{* 0}}\right)^{-1 / \gamma}
$$

donde $\left(p_{* 0}, \rho_{* 0}\right)$ son los valores que definen el estado de una partícula atmosférica en cualquier etapa del proceso adiabático al que se somete la atmósfera. Supongamos que $\left(p_{* 0}, \rho_{* 0}\right)$ son los valores al inicio del proceso. Si la atmósfera está en equilibrio hidrostático en los estados inicial y final del proceso, entonces $\rho_{* 0}$, $p_{* 0}$ dependen de la posición "inicial” $z_{s 0}$ de la partícula y $\rho_{0}, p_{0}$ de su posición final " $z_{s}$ ", es decir,

$$
\rho_{0}^{-1}\left(z_{s}\right)=\rho_{0}^{-1}\left(z_{s 0}\right)\left[\frac{p_{0}\left(z_{s}\right)}{p_{0}\left(z_{s 0}\right)}\right]^{-1 / \gamma} .
$$

Integrando obtenemos

$$
P\left[p_{0}\right]=c_{p} T_{0}\left(z_{s 0}\right)\left(\frac{p_{0}\left(z_{s}\right)}{p_{0}\left(z_{s 0}\right)}\right)^{R / c_{p}}
$$


Pero, de acuerdo con la fórmula de Poisson (1.39), la temperatura de una partícula "al final del proceso adiabático" está dada por

$$
T_{0}\left(z_{s}\right)=T_{0}\left(z_{s 0}\right)\left(\frac{p_{0}\left(z_{s}\right)}{p_{0}\left(z_{s 0}\right)}\right)^{R / c_{p}}
$$

entonces

$$
P\left[p_{0}\right]=c_{p} T_{0}\left(z_{s}\right)
$$

Considerando que las partículas en la superficie terrestre $\left(z_{s}=0\right)$ no se mueven durante todo el proceso y suponemos que al final del proceso las partículas en la superficie terrestre tienen los valores de referencia $p_{00}$, $T_{00}, \rho_{00}$, tenemos

$$
P\left[p_{00}\right]=c_{p} T_{00}
$$

donde suponemos que al final del proceso las partículas en la superficie terrestre tienen los valores de referencia $p_{00}, T_{00}, \rho_{00}$. Sustituyendo en (3.48) obtenemos la distribución de temperatura en una atmósfera de referencia adiabática

$$
T_{0}\left(z_{s}\right)=T_{00}-\frac{g a}{c_{p}}\left(1-\frac{a}{r}\right) .
$$

En términos de la altura característica de una atmósfera adiabática

$$
H_{s}=\frac{c_{p} T_{00}}{g} \sim \frac{104 \mathrm{~J}^{\circ} \mathrm{Kkg}^{-1} \times 300^{\circ} \mathrm{k}}{9.8 \mathrm{~ms}^{-1}}=30.735 \mathrm{~km} .
$$

podemos escribir

$$
T_{0}\left(z_{s}\right)=T_{00}\left[1-\frac{a}{H_{s}}\left(1-\frac{a}{r}\right)\right] .
$$

Para obtener la distribución de presión usamos (3.51),

$$
T_{0}\left(z_{s 0}\right)\left(\frac{p_{0}\left(z_{s}\right)}{p_{0}\left(z_{s 0}\right)}\right)^{R / c_{p}}=T_{00}\left[1-\frac{a}{H_{s}}\left(1-\frac{a}{r}\right)\right],
$$

de donde se obtiene

$$
p_{0}\left(z_{s}\right)=p_{0}\left(z_{s 0}\right)\left\{\frac{T_{00}}{T_{0}\left(z_{s 0}\right)}\left[1-\frac{a}{H_{s}}\left(1-\frac{a}{r}\right)\right]\right\}^{c_{p} / R} .
$$

Una aproximación consiste en sustituir $T_{0}\left(z_{s 0}\right)$ por el valor de referencia $T_{00}$, con lo cual se obtiene

$$
p_{0}\left(z_{s}\right)=p_{00}\left[1-\frac{a}{H_{s}}\left(1-\frac{a}{r}\right)\right]^{c_{p} / R}
$$

La aproximación $T_{0}\left(z_{s 0}\right) \sim T_{00}$ equivale a decir que inicialmente la atmósfera es isotérmica o que sólo consideramos en una pequeña capa atmosférica cercana a la superficie (probablemente no mayor a $100 \mathrm{~m}$ ), por lo que el uso de (3.54) para definir un estado de referencia en la troposfera puede se una mala aproximación.

\subsubsection{Estados de referencia locales}

Las expresiones (3.49-3.54) pueden simplificarse si consideramos que la relación $\left|z_{s}\right| \leq 20 \mathrm{~km}<<a$ tiene lugar en la troposfera. En tal caso tenemos

$$
1-\frac{a}{r}=1-\frac{a}{z_{s}+a}=1-\left(1+\frac{z_{s}}{a}\right)^{-1} \sim 1-\left(1-\frac{z_{s}}{a}\right)=\frac{z_{s}}{a}
$$

Así, para una atmósfera isotérmica tenemos

$$
p_{0}\left(z_{s}\right)=p_{00} e^{-z_{s} / H}
$$


Para el caso adiabático tenemos

$$
\begin{array}{ll}
p_{0}\left(z_{s}\right)=p_{00}\left\{\frac{T_{00}}{T_{0}\left(z_{s 0}\right)}\left[1-\frac{z_{s}}{H_{s}}\right]\right\}^{c_{p} / R}, \\
p_{0}\left(z_{s}\right)=p_{00} \quad\left[1-\frac{z_{s}}{H_{s}}\right]^{c_{p} / R} & \text { para } T_{0}\left(z_{s 0}\right) \sim T_{00} .
\end{array}
$$

Si usamos la fórmula de Poisson (3.51) y (3.54) para obtener la distribución de densidad tenemos

$$
\begin{gathered}
\rho_{0}\left(z_{s}\right)=\rho_{0}\left(z_{s 0}\right)\left\{\frac{T_{00}}{T_{0}\left(z_{s 0}\right)}\left[1-\frac{z_{s}}{H_{s}}\right]\right\}^{\frac{c_{v}}{R}}, \\
\rho_{0}\left(z_{s}\right)=\rho_{00}\left(z_{s 0}\right)\left(1-\frac{z_{s}}{H_{s}}\right)^{\frac{c_{v}}{R}} . \\
\text { para } z_{s 0} \sim 0
\end{gathered}
$$

Consideremos las expresiones anteriores en un sistema cartesiano asociado a un plano tangente a la esfera terrestre. Como se ve en la figura. 3.2 tenemos

$$
r=\sqrt{x^{2}+y^{2}+(z+a)^{2}}
$$

cualquiera que sea la orientación de los ejes $x, y$. El uso de esta expresión en (3.49-3.54) da los valores de referencia en un sistema cartesiano. Las expresiones resultantes pueden simplificarse con las consideraciones geométricas siguientes. Sea $L=\operatorname{máx}\{|x|,|y|\}$ Podemos estimar una cota de $L$ a partir de la intersección de la Troposfera y el plano $x y$. Sea $H_{0}$ la altura media de la troposfera. Como se observa en la figura 3.3 tenemos $r^{2}=\left(H_{0}+a\right)^{2}=2 L^{2}+a^{2}$ de donde se obtiene

$$
L^{2}=\frac{\left(H_{0}+a\right)^{2}-a^{2}}{2}=H_{0}\left(a+\frac{H_{0}}{2}\right)
$$

Para $H_{0} \sim 20 \mathrm{~km}$ y $a \sim 6378 \mathrm{~km}$ tenemos

$$
L=357,4 \mathrm{~km}
$$

valor que define el dominio máximo en el plano $x y[-L, L] \times[-L, L]$ sobre el cual la troposfera queda arriba de dicho dominio Con los valores de $L$ y $H_{0}$ podemos aproximar $r$. En términos de las variables adimensionales $\bar{x}=\frac{x}{L}, \bar{y}=\frac{y}{L}, \bar{z}=\frac{z}{L}$ tenemos

$$
r^{2}=\left(\bar{x}^{2}+\bar{y}^{2}\right) L^{2}+\left(a+H_{0} \bar{z}\right)^{2}=\bar{\xi} \frac{L^{2}}{a^{2}}+a^{2}\left(1+\bar{z} \frac{z_{\text {máx }}}{a}\right)^{2}=a^{2}\left[1+2 \bar{z} \frac{H_{0}}{a}+\bar{\xi} \frac{L^{2}}{a^{2}}+\bar{z}^{2} \frac{H_{0}^{2}}{a^{2}}\right]
$$

donde definimos

$$
\bar{\xi}^{2}=\bar{x}^{2}+\bar{y}^{2} .
$$

De acuerdo con el orden de magnitud de los cocientes

$$
\begin{aligned}
\frac{L}{a} & =\frac{357,4}{6378}=5,6 \times 10^{-2} \rightarrow \frac{L^{2}}{a^{2}}=3,1 \times 10^{-3} \\
\frac{H_{0}}{a} & =\frac{20}{6378}=3,1 \times 10^{-3} \rightarrow \frac{H_{0}^{2}}{a^{2}}=9,6 \times 10^{-6} \sim 10^{-5}
\end{aligned}
$$

podemos usar la aproximación

$$
r^{2} \sim a^{2}[1+\lambda], \quad \text { con } \quad \lambda \equiv 2 \bar{z} \frac{H_{0}}{a}+\bar{\xi}^{2} \frac{L^{2}}{a^{2}}
$$

con la cual obtenemos

$$
1-\frac{a}{r} \sim 1-(1+\lambda)^{-\frac{1}{2}} \sim 1-\left[1-\frac{\lambda}{2}\right]=\frac{\lambda}{2}=\frac{\bar{\xi}^{2}}{2} \frac{L^{2}}{a^{2}}+\frac{H_{0}}{a} \bar{z}=\frac{1}{a^{2}}\left(\frac{\xi^{2}}{2}+a z\right) .
$$


donde $\xi^{2}=x^{2}+y^{2}$. Sustituyendo en (3.49-3.54) obtenemos las siguientes expresiones válidas para $|x|$, $|y| \leq 357.4 \mathrm{~km},|z| \leq H_{0}=20 \mathrm{~km}, z_{s 0} \sim 0$ :

(i) Para una atmósfera de referencia isotérmica

$$
p_{0}(x, y, z)=p_{00} \exp \left[-\frac{1}{H a}\left(\frac{\xi^{2}}{2}+a z\right)\right], \quad \rho_{0}(x, y, z)=\rho_{00} \exp \left[-\frac{1}{a H}\left(\frac{\xi^{2}}{2}+a z\right)\right],
$$

(ii)Para una atmósfera adiabática

$$
p_{0}\left(z_{s}\right)=p_{00}\left[1-\frac{1}{a H_{s}}\left(\frac{\xi^{2}}{2}+a z\right)\right]^{c_{p} / R}, \quad \rho_{0}\left(z_{s}\right)=\rho_{00}\left[1-\frac{1}{a H_{s}}\left(\frac{\xi^{2}}{2}+a z\right)\right]^{c_{v} / R}
$$

En particular, para $\xi \sim 0$ obtenemos las expresiones reportadas en la literatura para el caso isotérmico

$$
p_{0}=p_{00} e^{-z / H} \quad \rho_{0}(x, y, z)=\rho_{00} e^{-z / H},
$$

y adiabático

$$
p_{0}\left(z_{s}\right)=p_{00}\left(1-\frac{z}{H_{s}}\right)^{c_{p} / R}, \quad \rho_{0}\left(z_{s}\right)=\rho_{00}\left(1-\frac{z}{H_{s}}\right)^{c_{v} / R}
$$




\section{Capítulo 4}

\section{Solución analítica de la ecuación de continuidad profunda}

El objetivo de este capítulo es construir un campo vectorial sintético que satisfaga

$$
\nabla \cdot \rho(\mathbf{r}) \mathbf{W}=0
$$

con las condiciones de frontera

$$
\mathbf{W} \cdot \mathbf{n}=0 \quad \text { sobre } \quad z=h(x)
$$

donde $h(x)$ es la elevación del terreno y $\mathbf{n}$ es un vector normal a ésta. Este campo será utilizado en los capítulos siguientes para dos cosas:

1. La componente horizontal de $\mathbf{W}$ será el campo inicial $\mathbf{v}^{0}$, ahorrándonos la penosa y costosa tarea de obtener los datos reales.

2. Como $\mathbf{W}$ es la solución analítica podremos evaluar de manera confiable la bondad de el método variacional que se discutirá más adelante.

El primer paso es encontrar la solución de la ecuación

$$
\nabla \cdot \mathbf{v}(\mathbf{r})=0
$$

con la condición de frontera

$$
\mathbf{v}(\mathbf{r}) \cdot \mathbf{n}=0 \quad \text { sobre } \quad z=h(x) .
$$

Una vez conocido el campo $\mathbf{v}$, éste satisface

$$
\nabla \cdot \rho(\mathbf{r}) \frac{\mathbf{v}(\mathbf{r})}{\rho(\mathbf{r})}=0
$$

con la condición de frontera

$$
\frac{\mathbf{v}(\mathbf{r})}{\rho(\mathbf{r})} \cdot \mathbf{n}=0 \quad \text { sobre } \quad z=h(x) .
$$

Por lo tanto

$$
\mathbf{W}(\mathbf{r})=\frac{\mathbf{v}(\mathbf{r})}{\rho(\mathbf{r})}
$$

es una solución de la ecuación (4.1). 


\subsection{Cálculo de $\mathrm{v}(\mathrm{r})$ con el método de mapeo conforme}

El método consiste en transformar el semiplano superior de un espacio abstracto $(\zeta \geq 0)$ en otra región del espacio físico con topografía arbitraria $h(x)$. Sea un punto del espacio físico

$$
\alpha=x+i z
$$

y el correspondiente punto en el espacio abstracto

$$
\beta=\chi+i \zeta
$$

tales que

$$
\alpha=G(\beta)
$$

donde $G$ es una función analítica de $\beta$. Como queremos mapear el eje $\zeta=0$ en la topografía, entonces, la transformación es

$$
G(\chi)=x+i h(x)
$$

Si pedimos que $x=\chi$ entonces

$$
G(\chi)=\chi+i h(\chi)
$$

Es claro que la función

$$
G(\beta)=\beta+i h(\beta)
$$

tiene la propiedad de mapear el eje real del espacio abstracto en la topografía. Si separamos a $h(\beta)$ en su componente real e imaginaria tenemos

$$
h(\beta)=h_{1}(\chi, \zeta)+i h_{2}(\chi, \zeta)
$$

entonces

$$
G(\beta)=\chi-h_{2}(\chi, \zeta)+i\left[\zeta+h_{1}(\chi, \zeta)\right]
$$

Comparando con (4.5) obtenemos el sistema de ecuaciones

$$
\begin{aligned}
& x=\chi-h_{2}(\chi, \zeta) \\
& z=\zeta+h_{1}(\chi, \zeta) .
\end{aligned}
$$

con el que definimos la trasformación de las coordenadas del espacio abstracto a las del espacio físico. El grado de dificultad con la que obtendremos la solución del sistema (4.9) dependerá en gran medida de las funciones $h_{1}(\chi, \zeta)$ y $h_{2}(\chi, \zeta)$. Para ilustrar lo anterior, usemos la topografía

$$
h(x)=\operatorname{sen}\left(\frac{\pi}{a} x\right)
$$

La transformación correspondiente es

$$
\begin{aligned}
& x=\chi-\cos \left(\frac{\pi}{a} \chi\right) \operatorname{senh}\left(\frac{\pi}{a} \zeta\right) \\
& z=\zeta+\operatorname{sen}\left(\frac{\pi}{a} \chi\right) \cosh \left(\frac{\pi}{a} \zeta\right)
\end{aligned}
$$

La presencia de las funciones hiperbólicas implica que una región en el plano $z$ que puede ser modificada drásticamente por tal transformación. La figura 4.2 muestra que la imagen del semiplano $\zeta \geq 0$ bajo la transformación es una región muy pequeña del espacio físico. Esto quiere decir que no podremos calcular el campo en una región arbitraria de el espacio físico. Una forma de evitar tal problema es representar a la topografía por medio de polinomios de bajo orden. Esto nos proporciona expresiones más simples con las cuales podremos calcular el campo en regiones arbitrarias. 


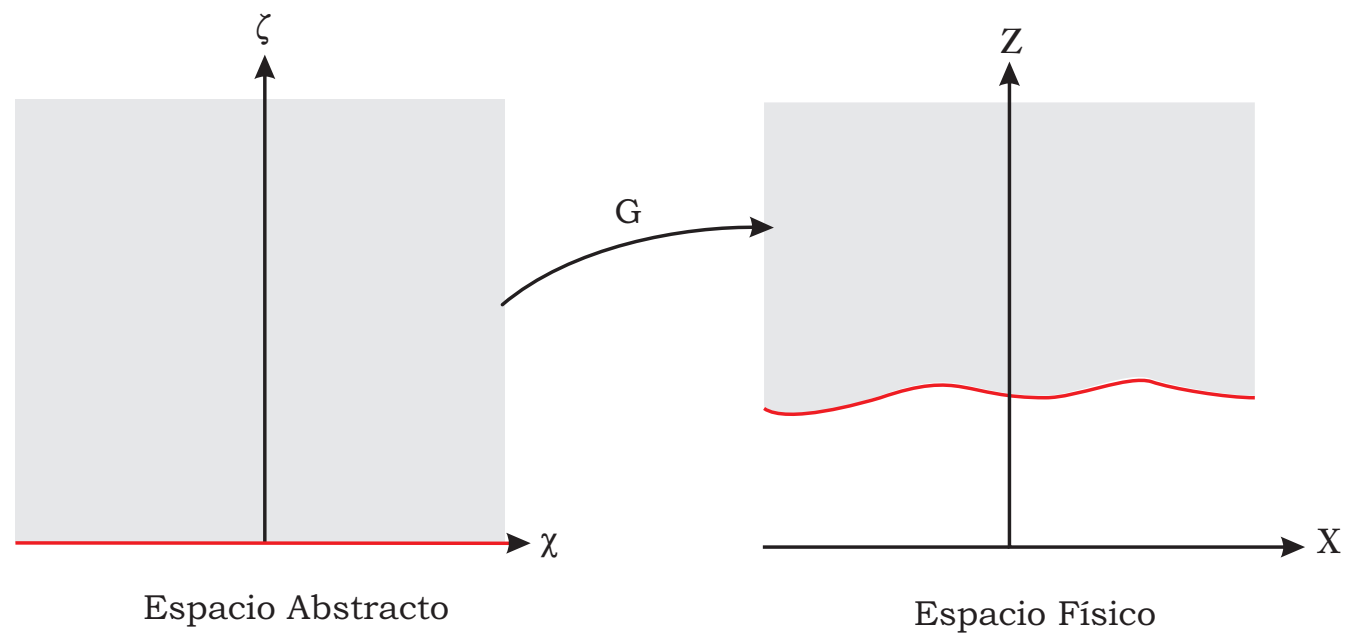

Figura 4.1: Transformación de el semiplano superior $\zeta \geq 0$ en la región $z \geq h(x)$.

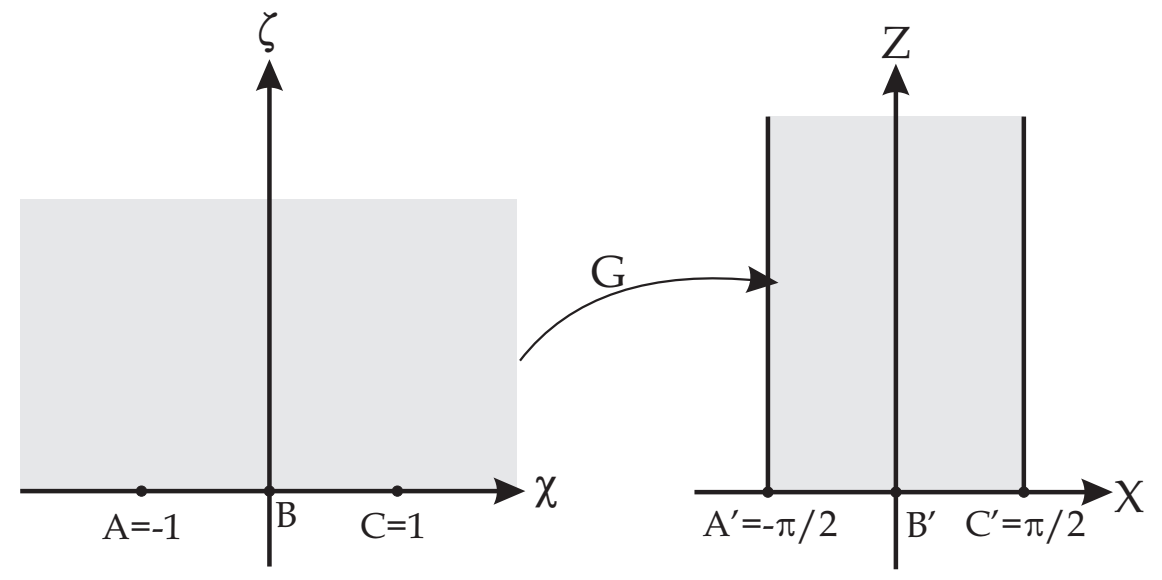

Figura 4.2: Deformación del espacio abstracto inducido por la transformación (4.10) 


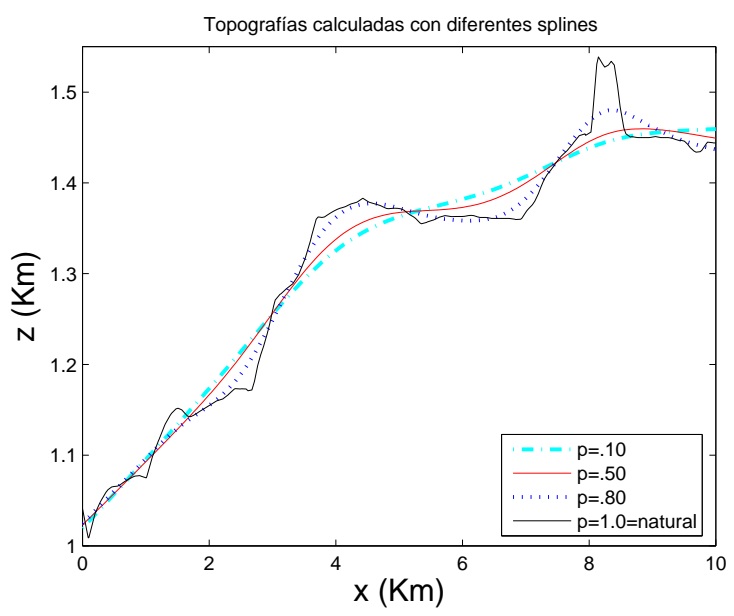

Figura 4.3: Comparación de diferentes factores de suavizamiento de la interpolación de los datos de la topografía.

\subsection{Interpolación Polinomial Cúbica Segmentada $\left(\operatorname{IP}^{3} \mathrm{~S}\right)$ Natural}

Un método para obtener una representación sencilla de la topografía es la Interpolación Polinomial Segmentada que consiste en ajustar un polinomio de grado $m$ sólo a un par de datos, por lo que la curva que ajusta a los $N$ datos está formada de $N-1$ polinomios de grado $m$. En este caso utilizaremos polinomios cúbicos para obtener la expresión de la topografía en el espacio físico. Tales polinomios tienen la expresión siguiente:

$$
\begin{aligned}
h_{k}(x) & =a_{k}+b_{k}\left(x-x_{k}\right)+c_{k}\left(x-x_{k}\right)^{2}+d_{k}\left(x-x_{k}\right)^{3} \\
x & \in\left[x_{k}, x_{k+1}\right] \quad k=1, \ldots, N-1 .
\end{aligned}
$$

donde el subíndice $k$ indica el $k$-ésimo dato. Adicionalmente se imponen $4(N-1)$ condiciones

$$
\begin{array}{rlrl}
h_{k}\left(x_{k}\right) & = & h\left(x_{k}\right) & k=1, \ldots, N-1 \\
h_{N-1}\left(x_{N}\right) & = & h\left(x_{N}\right) \\
h_{k}\left(x_{k+1}\right) & = & h_{k+1}\left(x_{k+1}\right) & k=1, \ldots, N-2 \\
h_{k}^{\prime}\left(x_{k+1}\right) & = & h_{k+1}^{\prime}\left(x_{k+1}\right) & k=1, \ldots, N-2 \\
h_{k}^{\prime \prime}\left(x_{k+1}\right) & = & h_{k+1}^{\prime \prime}\left(x_{k+1}\right) \quad k=1, \ldots, N-2 . \\
h_{1}^{\prime \prime}\left(x_{1}\right) & = & h_{N-1}^{\prime \prime}\left(x_{N}\right)=0
\end{array}
$$

que son necesarias para determinar los $4(N-1)$ coeficientes. A este tipo de polinomios se les conoce como polinomios cúbicos naturales y tiene la característica de que los polinomios pasan por los datos, dando a la curva que ajusta a los datos de la topografía un aspecto muy parecido a la topografía real, sin embargo, el campo de vientos resultante es errático, lo cual hace necesario suavizar la topografía. A continuación se describirá cómo construir los polinomios que forman la interpolación. De (4.11) es fácil ver que

$$
h_{k}\left(x_{k}\right)=a_{k} .
$$

Entonces de (4.12c), de la ecuación anterior y de la siguiente definición

$$
\delta_{k}=x_{k+1}-x_{k}
$$

obtenemos la siguiente ecuación

$$
a_{k+1}=a_{k}+b_{k} \delta_{k}+c_{k} \delta_{k}+d_{k} \delta_{k}^{3} .
$$


Además, de (4.12d) y de (4.12e) obtenemos las siguientes ecuaciones

$$
\begin{aligned}
& b_{k+1}=b_{k}+2 c_{k} \delta_{k}+3 d_{k} \delta_{k}^{2} \\
& c_{k+1}=c_{k}+3 d_{k} \delta_{k} .
\end{aligned}
$$

Al eliminar los coeficientes $b$ y $d$ de (4.13), (4.14) y (4.15) obtenemos el siguiente sistema de ecuaciones para los coeficientes $c$

$$
\delta_{k-1} c_{k-1}+2 c_{k}\left(\delta_{k-1}+\delta_{k}\right)+\delta_{k} c_{k+1}=\frac{3}{\delta_{k}}\left(a_{k+1}-a_{k}\right)-\frac{3}{\delta_{k-1}}\left(a_{k}-a_{k-1}\right) .
$$

Los demás coeficientes se calculan con las siguientes ecuaciones

$$
\begin{aligned}
b_{k} & =\frac{1}{\delta_{k}}\left(a_{k}-a_{k-1}\right)-\frac{1}{3} \delta_{k}\left(c_{k+1}+2 c_{k}\right) \\
d_{k} & =\frac{1}{3 \delta_{k}}\left(c_{k+1}-c_{k}\right) .
\end{aligned}
$$

Para que las expresiones de las secciones siguientes sean más compactas realizaremos el siguiente cambio de variable

$$
\begin{aligned}
a_{k}^{\prime} & =a_{k}-b_{k} h\left(x_{k}\right)+c_{k} h^{2}\left(x_{k}\right)-d_{k} h^{3}\left(x_{k}\right) \\
b_{k}^{\prime} & =b_{k}-2 c_{k} h\left(x_{k}\right)+3 d_{k} h^{2}\left(x_{k}\right) \\
c_{k}^{\prime} & =c_{k}-3 d_{k} h\left(x_{k}\right) .
\end{aligned}
$$

Con esto, la expresión de los polinomios cúbicos queda de manera más simple

$$
h_{k}(x)=a_{k}^{\prime}+b_{k}^{\prime} x+c_{k}^{\prime} x^{2}+d_{k}^{\prime} x^{3}
$$

\subsection{Interpolación Polinomial Cúbica Segmentada Suavizada}

Este tipo de interpolación consiste en encontrar los coeficientes de los $N-1$ polinomios cúbicos tales que minimicen el funcional

$$
p \sum_{i=1}^{N}\left[\frac{y_{i}-h\left(x_{i}\right)}{\sigma_{i}}\right]^{2}+(1-p) \int_{x_{1}}^{x_{N}}\left[h^{\prime \prime}(\tau)\right]^{2} d \tau
$$

donde $p \in[0,1], h(x)=\left\{h_{k}(x) \mid x \in\left[x_{k}, x_{k+1}\right] ; k=1,2, \ldots, N-1\right\}$ y $\sigma_{i}$ es la desviación estándar de el dato $y_{i}$. En este funcional están presentes dos aspectos importantes de la interpolación: en el primer término se exige que $h(x)$ esté lo más cerca posible de los datos, mientras que en el segundo término se pide que $h(x)$ sea una función suave. La manera en que podemos dar mayor importancia a alguno de los términos es a través de el parámetro $p$, por ejemplo, con $p=0$ obtendremos una función $h(x)$ suave, aunque es probable que no pase cerca de los puntos $y_{i}$. En cambio con $p=1$ la función $h(x)$ pasará por los puntos, sin embargo, no debemos esperar que sea una función suave.

Para resolver el problema discretizamos (4.19). El primer término no representa mayor problema, por lo que nos dedicaremos a la discretización de la integral. Ya que la segunda derivada de un polinomio cúbico resulta ser una línea recta $l(x)=m x+b$ la integral puede ser evaluada analíticamente:

$$
\int_{0}^{x}[l(\tau)]^{2} d \tau=\frac{1}{3} x\left[m^{2} x^{2}+3 m b x+3 b^{2}\right] .
$$

El lado derecho se puede desarrollar de tal manera que tenemos

$$
\begin{aligned}
\int_{0}^{x}[l(\tau)]^{2} d \tau & =\frac{1}{3} x[\underbrace{m^{2} x^{2}+2 m b x+b^{2}}_{[l(x)]^{2}}+\underbrace{b(m x+b)}_{l(0) l(x)}+\underbrace{b^{2}}_{[l(0)]^{2}}] \\
& =\frac{1}{3} x\left[[l(x)]^{2}+l(x) l(0)+[l(0)]^{2}\right]
\end{aligned}
$$


es decir, la integral sólo depende del ancho del intervalo de integración y de el valor de la segunda derivada en los extremos de cada intervalo $\left(h^{\prime \prime}\left(x_{i}\right)=2 c_{i}\right)$, por lo que, en general la discretización de (4.19) está dada por

$$
p \sum_{i=1}^{N}\left[\frac{y_{i}-a_{i}}{\sigma_{i}}\right]^{2}+\frac{4(1-p)}{3} \sum_{k=1}^{N-1} \delta x_{i}\left(c_{i}^{2}+c_{i} c_{i+1}+c_{i+1}^{2}\right) .
$$

Minimizando esta ecuación y con (4.16) podemos obtener el sistema de ecuaciones para los coeficientes $c_{i}$, que en forma matricial es

$$
\left[6(1-p) \mathbb{Q}^{T} \mathbb{D}^{2} \mathbb{Q}+p \mathbb{R}\right] \mathbf{c}=3 p \mathbb{Q}^{T} \mathbf{y}
$$

Donde $\mathbb{Q}_{(N-2) \times N}$ es una matriz tridiagonal cuyo i-ésimo renglón es

$$
\frac{1}{\delta x_{i-1}},-\frac{1}{\delta x_{i-1}}-\frac{1}{\delta x_{i}}, \frac{1}{\delta x_{i}},
$$

$\mathbb{R}_{N-2 \times N-2}$ es una matriz simétrica tridiagonal con su i-ésimo renglón

$$
\delta x_{i-1}, 2\left(\delta x_{i-1}+\delta x_{i}\right), \delta x_{i}
$$

y $\mathbb{D}$ es una matriz diagonal cuyos elementos son las desviaciones estándar de los datos $y_{i}$. Los detalles de como llegar a el sistema (4.21) se encuentran en [3, p235-243]. Una vez obtenidos los coeficientes $c_{i}$ se pueden calcular los demás coeficientes usando (4.17) y (4.18) con lo que habremos resuelto el problema de encontrar una representación analítica de la topografía.

\subsection{Expresión explícita del campo $\mathrm{v}$}

De los resultados de las secciones anteriores y de la ecuación (4.9) tenemos que las partes, real e imaginaria, de la topografía son

$$
\begin{aligned}
& h_{1 k}(\chi, \zeta)=a_{k}+b_{k} \chi+c_{k}\left(\chi^{2}-\zeta^{2}\right)+d_{k} \chi\left(\chi^{2}-3 \zeta^{2}\right) \\
& h_{2 k}(\chi, \zeta)=b_{k} \zeta+2 c_{k} \chi \zeta+d_{k} \zeta\left(3 \chi^{2}-\zeta^{2}\right) .
\end{aligned}
$$

Por lo que la transformación inversa $G^{-1}(\alpha)=\beta$ puede calcularse al resolver el sistema de ecuaciones no lineales

$$
\begin{aligned}
x & =\chi-\left[b_{k} \zeta+2 c_{k} \chi \zeta+d_{k} \zeta\left(3 \chi^{2}-\zeta^{2}\right)\right] \\
z & =\zeta+a_{k}+b_{k} \chi+c_{k}\left(\chi^{2}-\zeta^{2}\right)+d_{k} \chi\left(\chi^{2}-3 \zeta^{2}\right)
\end{aligned}
$$

en donde las incógnitas son $\chi$ y $\zeta$. Con la transformación ya definida podemos calcular el campo de velocidad. Sea un potencial complejo en el espacio físico

$$
F(x, z)=\phi(x, z)+i \psi(x, z)
$$

tal que

$$
u=\frac{\partial \phi}{\partial x}=\frac{\partial \psi}{\partial z} \quad w=\frac{\partial \phi}{\partial z}=-\frac{\partial \psi}{\partial x}
$$

donde $u$ y $v$ son las componentes de un campo de velocidad que satisface la ecuación (4.2) y las condiciones (4.3). Utilizando la regla de la cadena tenemos

$$
\begin{aligned}
& u=\frac{\partial \phi}{\partial x}=\frac{\partial \chi}{\partial x} \frac{\partial \phi}{\partial \chi}+\frac{\partial \zeta}{\partial x} \frac{\partial \phi}{\partial \zeta} \\
& w=\frac{\partial \phi}{\partial z}=\frac{\partial \chi}{\partial z} \frac{\partial \phi}{\partial \chi}+\frac{\partial \zeta}{\partial z} \frac{\partial \phi}{\partial \zeta}
\end{aligned}
$$

en donde

$$
\frac{\partial \phi}{\partial \chi}=\nu \quad \frac{\partial \phi}{\partial \zeta}=\omega
$$


son las componentes de el campo en el espacio abstracto. En forma matricial tenemos

$$
\left(\begin{array}{c}
u \\
w
\end{array}\right)=\left(\begin{array}{ll}
\frac{\partial \chi}{\partial x} & \frac{\partial \zeta}{\partial x} \\
\frac{\partial \chi}{\partial z} & \frac{\partial \zeta}{\partial z}
\end{array}\right)\left(\begin{array}{l}
\nu \\
\omega
\end{array}\right)=\left(\mathbb{J}^{-1}\right)^{T}\left(\begin{array}{l}
\nu \\
\omega
\end{array}\right)=\frac{1}{J}\left(\begin{array}{cc}
\frac{\partial z}{\partial \zeta} & -\frac{\partial z}{\partial \chi} \\
-\frac{\partial x}{\partial \zeta} & \frac{\partial x}{\partial \chi}
\end{array}\right)\left(\begin{array}{l}
\nu \\
\omega
\end{array}\right)
$$

donde

$$
\mathbb{J}=\left(\begin{array}{ll}
\frac{\partial x}{\partial \chi} & \frac{\partial x}{\partial \zeta} \\
\frac{\partial z}{\partial \chi} & \frac{\partial z}{\partial \zeta}
\end{array}\right)
$$

es la matriz jacobiana calculada con (4.9) y $J$ es el determinante de $\mathbb{J}$. Tomando un campo de velocidad uniforme en el espacio abstracto tal que

$$
\begin{aligned}
& \nu=\nu_{0} \\
& \omega=0 .
\end{aligned}
$$

obtenemos la expresión del campo $\mathbf{v}$ en términos de los coeficientes de los polinomios cúbicos y de la componente horizontal del campo de velocidad en el espacio abstracto

$$
\begin{aligned}
u_{k} & =\frac{\nu_{0}}{J}\left[1-2 \zeta\left(c_{k}+3 d_{k} \chi\right)\right] \\
w_{k} & =\frac{\nu_{0}}{J}\left[b_{k}+2 c_{k} \chi+3 d_{k}\left(\chi^{2}-\zeta^{2}\right)\right] .
\end{aligned}
$$

En donde el determinante de $\mathbb{J}$ está dado por

$$
J=\frac{\partial x}{\partial \chi} \frac{\partial z}{\partial \zeta}-\frac{\partial x}{\partial \zeta} \frac{\partial z}{\partial \chi}
$$

Las derivadas parciales involucradas en el cálculo del Jacobiano son

$$
\begin{aligned}
& \frac{\partial x}{\partial \chi}=1-\frac{\partial h_{2}}{\partial \chi}=1-2 \zeta\left(c_{k}+3 d_{k} \chi\right) \\
& \frac{\partial z}{\partial \zeta}=1+\frac{\partial h_{1}}{\partial \zeta}=1-2 \zeta\left(c_{k}+3 d_{k} \chi\right) \\
& \frac{\partial x}{\partial \zeta}=-\frac{\partial h_{2}}{\partial \zeta}=-\left[b_{k}+2 c_{k} \chi+3 d_{k}\left(\chi^{2}-\zeta^{2}\right)\right] \\
& \frac{\partial z}{\partial \chi}=\frac{\partial h_{1}}{\partial \chi}=b_{k}+2 c_{k} \chi+3 d_{k}\left(\chi^{2}-\zeta^{2}\right) .
\end{aligned}
$$

Para simplificar la notación sean

$$
J_{11}=1-2 \zeta\left(c_{k}+3 d_{k} \chi\right) \quad \text { y } \quad J_{21}=b_{k}+2 c_{k} \chi+3 d_{k}\left(\chi^{2}-\zeta^{2}\right),
$$

entonces el Jacobiano es

$$
J=J_{11}^{2}+J_{21}^{2}
$$

De aquí se puede ver que la transformación está bien definida en $\left[x_{k}, x_{k+1}\right]$ pues el jacobiano nuca es cero y que el campo $\left(u_{k}, w_{v}\right)$ es analítico en cada intervalo.

Por otro lado la divergencia del campo v, generado con mapeo conforme, puede ser calculada de manera explícita a partir de las expresiones anteriores para verificar que cumple con la condición (4.2). Para calcular la divergencia sabemos que ésta es

$$
\nabla \cdot \mathbf{v}=\frac{\partial u_{k}}{\partial x}+\frac{\partial w_{k}}{\partial z}
$$

Ya que $u$ y $w$ son funciones de $\chi$ y $\zeta$ usaremos la regla de la cadena para calcular las derivadas parciales en términos de éstas variables, entonces 


$$
\begin{aligned}
\frac{\partial u_{k}}{\partial x} & =\frac{\partial u_{k}}{\partial \chi} \frac{\partial \chi}{\partial x}+\frac{\partial u_{k}}{\partial \zeta} \frac{\partial \zeta}{\partial x} \\
\frac{\partial w_{k}}{\partial z} & =\frac{\partial w_{k}}{\partial \chi} \frac{\partial \chi}{\partial z}+\frac{\partial w_{k}}{\partial \zeta} \frac{\partial \zeta}{\partial z}
\end{aligned}
$$

De (4.24) tenemos que

$$
\begin{array}{ll}
\frac{\partial \chi}{\partial x}=\frac{1}{J} \frac{\partial z}{\partial \zeta} & \frac{\partial \zeta}{\partial x}=-\frac{1}{J} \frac{\partial z}{\partial \chi} \\
\frac{\partial \chi}{\partial z}=-\frac{1}{J} \frac{\partial x}{\partial \zeta} & \frac{\partial \zeta}{\partial z}=\frac{1}{J} \frac{\partial x}{\partial \chi}
\end{array}
$$

o bien

$$
\begin{array}{ll}
\frac{\partial \chi}{\partial x}=\frac{1}{J} J_{11} & \frac{\partial \zeta}{\partial x}=-\frac{1}{J} J_{21} \\
\frac{\partial \chi}{\partial z}=\frac{1}{J} J_{21} & \frac{\partial \zeta}{\partial z}=\frac{1}{J} J_{11}
\end{array}
$$

Con (4.25) y (4.27) podemos expresar de manera más compacta las componentes del campo v:

$$
\begin{gathered}
u_{k}=\frac{\nu_{0}}{J} J_{11} \\
w_{k}=\frac{\nu_{0}}{J} J_{21} .
\end{gathered}
$$

Entonces, las derivadas parciales de las componentes del campo $\mathbf{v}$ respecto a las variables $\chi$ y $\zeta$ son

$$
\begin{aligned}
\frac{\partial u_{k}}{\partial \chi}=\nu_{0}\left\{\frac{1}{J} \frac{\partial J_{11}}{\partial \chi}-\frac{J_{11}}{J^{2}} \frac{\partial J}{\partial \chi}\right\}=\frac{1}{J}\left\{\nu_{0} \frac{\partial J_{11}}{\partial \chi}-u_{k} \frac{\partial J}{\partial \chi}\right\} \\
\frac{\partial u_{k}}{\partial \zeta}=\nu_{0}\left\{\frac{1}{J} \frac{\partial J_{11}}{\partial \zeta}-\frac{J_{11}}{J^{2}} \frac{\partial J}{\partial \zeta}\right\}=\frac{1}{J}\left\{\nu_{0} \frac{\partial J_{11}}{\partial \zeta}-u_{k} \frac{\partial J}{\partial \zeta}\right\} \\
\frac{\partial w_{k}}{\partial \chi}=\nu_{0}\left\{\frac{1}{J} \frac{\partial J_{21}}{\partial \chi}-\frac{J_{21}}{J^{2}} \frac{\partial J}{\partial \chi}\right\}=\frac{1}{J}\left\{\nu_{0} \frac{\partial J_{21}}{\partial \chi}-w_{k} \frac{\partial J}{\partial \chi}\right\} \\
\frac{\partial w_{k}}{\partial \zeta}=\nu_{0}\left\{\frac{1}{J} \frac{\partial J_{21}}{\partial \zeta}-\frac{J_{21}}{J^{2}} \frac{\partial J}{\partial \zeta}\right\}=\frac{1}{J}\left\{\nu_{0} \frac{\partial J_{21}}{\partial \zeta}-w_{k} \frac{\partial J}{\partial \zeta}\right\} .
\end{aligned}
$$

Para finalizar el cálculo de la divergencia del campo tenemos que las derivadas parciales de el Jacobiano y de las componentes de la matriz Jacobiana son

$$
\begin{aligned}
& \frac{\partial J}{\partial \chi}=2\left(J_{11} \frac{\partial J_{11}}{\partial \chi}+J_{21} \frac{\partial J_{21}}{\partial \chi}\right) \\
& \frac{\partial J}{\partial \zeta}=2\left(J_{11} \frac{\partial J_{11}}{\partial \zeta}+J_{21} \frac{\partial J_{21}}{\partial \zeta}\right) \\
& \frac{\partial J_{11}}{\partial \chi}=-6 d_{k} \zeta \quad \frac{\partial J_{21}}{\partial \chi}=2\left(c_{k}+3 d_{k} \chi\right) \\
& \frac{\partial J_{21}}{\partial \zeta}=-6 d_{k} \zeta \quad \frac{\partial J_{11}}{\partial \zeta}=-2\left(c_{k}+3 d_{k} \chi\right) .
\end{aligned}
$$

Por último, para verificar que el campo v satisface la condición (4.3) sabemos que el vector tangente a la topografía es 


$$
\mathbf{t}=\left(\begin{array}{c}
1 \\
h_{k}^{\prime}(x)
\end{array}\right)
$$

Entonces, el vector normal a la topografía es

$$
\mathbf{n}=\left(\begin{array}{c}
-h_{k}^{\prime}(x) \\
1
\end{array}\right)
$$

Por lo que el producto escalar de el campo $\mathbf{v}$ con el vector normal a la topografía es

$$
\mathbf{v} \cdot \mathbf{n}=-u h_{k}^{\prime}(x)+w
$$

donde

$$
h_{k}^{\prime}(x)=b_{k}+2 c_{k} x+3 d_{k} x^{2} .
$$

\subsection{Algoritmo del método de mapeo conforme}

De manera breve se presentan los pasos a seguir para calcular el campo $\mathbf{v}$ con mapeo conforme:

1. Lectura de los puntos que forman la topografía.

2. Lectura de los puntos en los que se quiere calcular el campo $\mathbf{v}$.

3. Lectura de los coeficientes de los polinomios cúbicos que interpolan la topografía.

4. Determinar el intervalo $\left[x_{k}, x_{k+1}\right]$ correspondiente a cada punto $(x, z)$ del inciso 2 . Con esto se sabe qué conjunto de coeficientes $\left\{a_{k}, b_{k}, c_{k}, d_{k}\right\}$ utilizar para calcular el campo en dicho punto.

5. Determinación del campo uniforme $\nu_{0}$. Esto puede hacerse de dos maneras. La primera es dar, simplemente, el valor de el campo uniforme y la segunda es dar el valor de la componente horizontal del campo $\mathbf{v}$ medido en algún punto $(x, z)$, resolver el sistema de ecuaciones no lineales (4.23) para obtener el punto $(\chi, \zeta)$ correspondiente en el espacio abstracto y, por último, despejar $\nu_{0}$ de $(4.25 \mathrm{a})$.

6. Solución del sistema de ecuaciones no lineales $(4.23)$ para obtener el punto $(\chi, \zeta)$ del espacio abstracto correspondientes con el punto $(x, z)$ en el espacio físico por medio del método de Newton. De aquí se obtienen las componentes de la matriz jacobiana y su determinante utilizados en el cálculo del campo y de su divergencia.

7. Con la información de el inciso anterior se calculan las componentes del campo $\mathbf{v}$ y se verifica que cumpla con las condiciones (4.2) y (4.3).

8. Cálculo de la densidad la cual puede ser constante o calculada con las relaciones

9. Registro de resultados.

\subsection{Efecto de la topografía en el campo v}

Los datos de la topografía con los que se desarrollaron los ejemplos fueron tomados de [41]. En las figuras (4.4 - 4.6) puede observarse que entre más suave sea la topografía, más suave será el campo. Por ejemplo, en la figura (4.4) se observa el campo interpolado y la topografía generada con las condiciones naturales (4.12). Como la topografía es irregular, el campo tiende a ser errático y es poco probable que este campo se pueda recuperar con el método variacional. Por otro lado, tenemos las figuras (4.5) y (4.6) en donde la topografía se ha generado con condiciones de suavizamiento (4.19). El campo en ambos casos es más suave que en el caso anterior. Este tipo de campos es más adecuado para ser utilizados en el método variacional pues, como se verá más adelante, el campo de velocidad recuperado con éste método es suave. Esto pone de manifiesto el papel que juega el parámetro de suavizamiento $p$. Con éste parámetro podemos controlar la suavidad del campo de vientos que utilizaremos como prueba del método variacional y así saber de manera precisa que tan bueno es. 

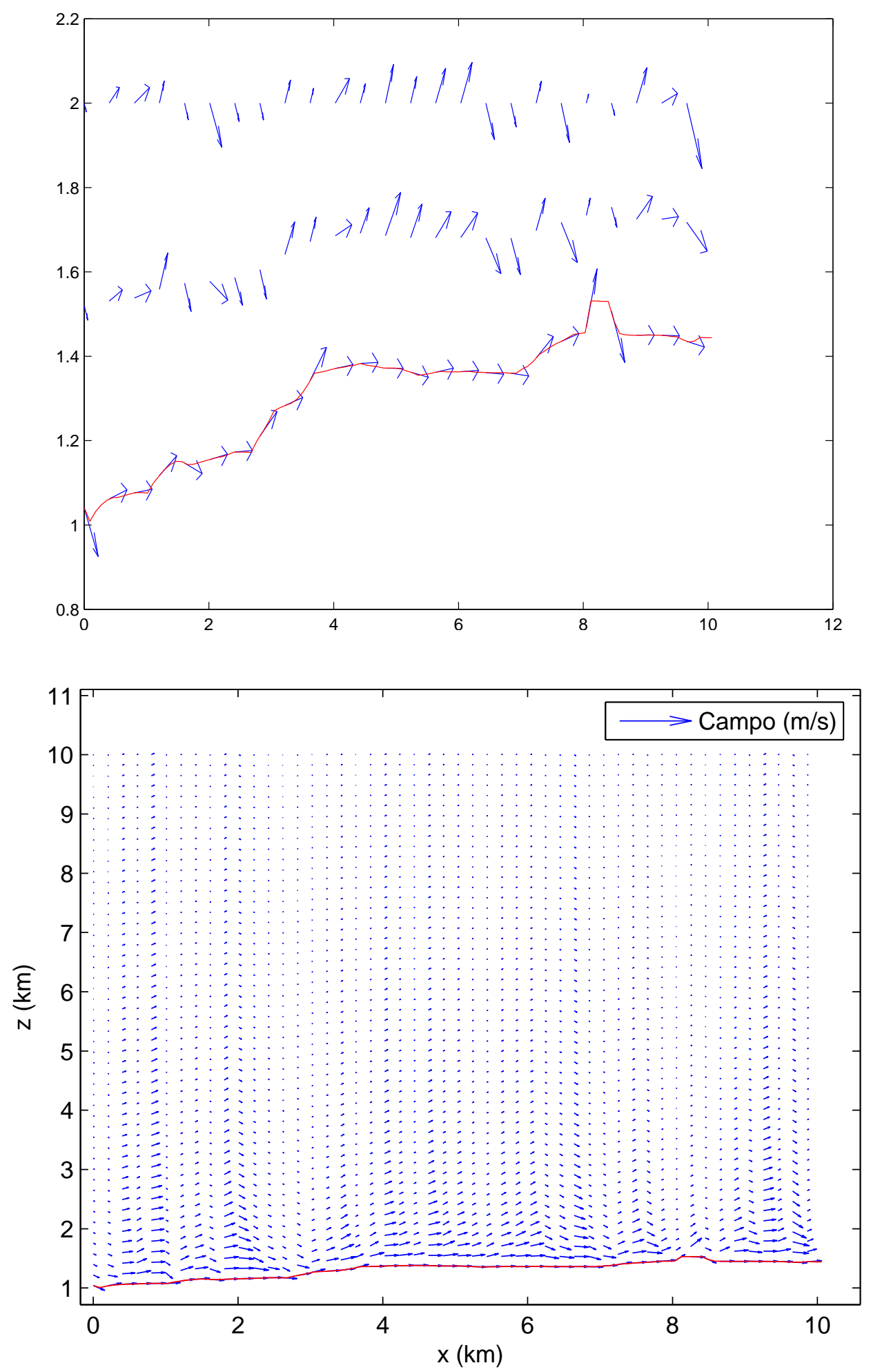

Figura 4.4: Campo de vientos calculado con mapeo conforme y la topografía ajustada con polinomios cúbicos con $\mathrm{p}=1.0$ 

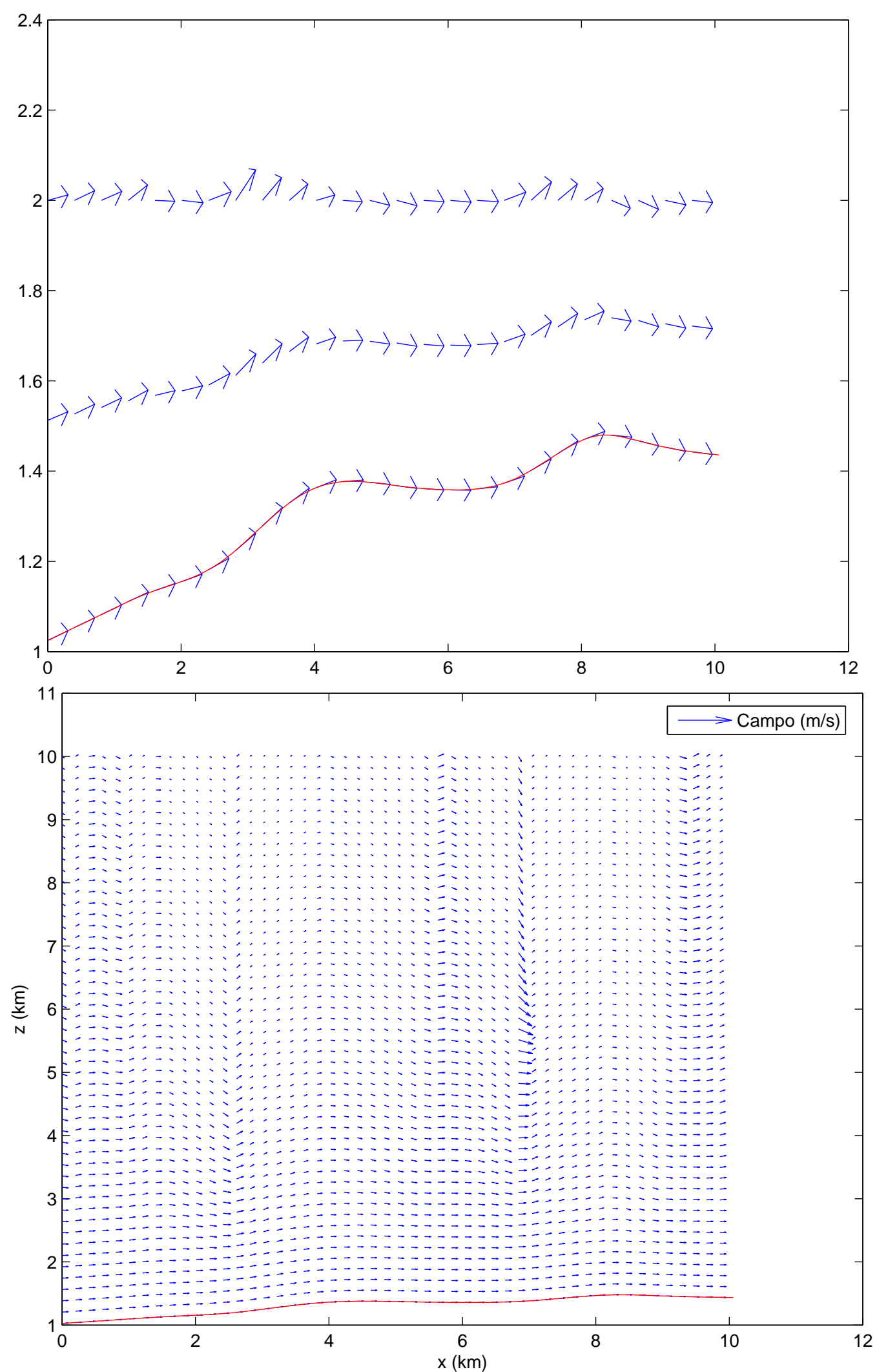

Figura 4.5: Campo de vientos calculado con mapeo conforme y la topografía ajustada con polinomios cúbicos con $\mathrm{p}=0.8$ 

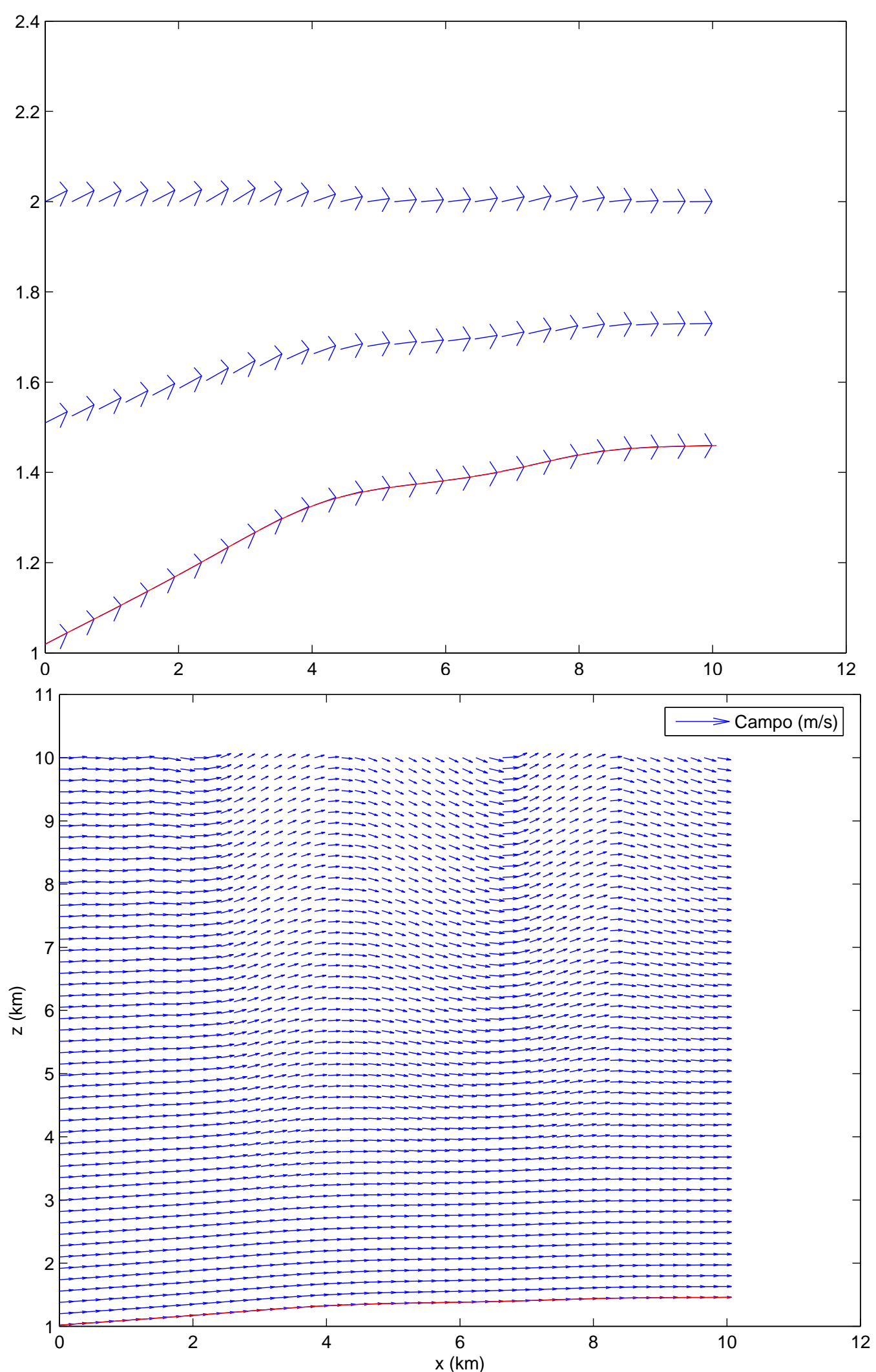

Figura 4.6: Campo de vientos calculado con mapeo conforme y la topografía ajustada con polinomios cúbicos con $\mathrm{p}=0.1$ 


\section{Capítulo 5}

\section{Cálculo aproximado de la solución de $\nabla \cdot \mathbf{v}=0$ a partir de un conjunto de datos experimentales}

En el presente capítulo se presentas las ideas desarrolladas en [25], [30] y [31], las gráficas y resultados numéricos se reproducen con el permiso de los autores.

\subsection{Planteamiento del problema. Campos inicial $\mathrm{v}^{0} \mathrm{y}$ ajustado $\mathrm{v}$}

Consideremos un sistema cartesiano $x y z$ con su origen en un punto con coordenadas geográficas $\left(\lambda_{c}, \phi_{c}\right)$ sobre un modelo esférico terrestre, el plano $x y$ es tangente a la esfera y el eje $z$ es exterior a la esfera (ver la figura 4.1). En lo que sigue usaremos: (i) en forma indistinta la notación $x^{1}=x, x^{2}=y, x^{3}=z \mathrm{y} \hat{\mathbf{x}}=\hat{\mathbf{x}}_{1}$, $\hat{\mathbf{y}}=\hat{\mathbf{x}}_{2}, \hat{\mathbf{z}}=\hat{\mathbf{x}}_{3}$ para las coordenadas y vectores unitarios asociados al sistema $x y z$, y (ii) la convención de suma sobre índices repetidos, de manera que $\sum_{i} v_{\text {real }}^{i} \hat{\mathbf{x}}_{i}=v_{\text {real }}^{i} \hat{\mathbf{x}}_{i}$. En el sistema $x y z$ definimos la región acotada

$$
\mathcal{D}=\left\{x_{\text {mín }} \leqslant x \leqslant x_{\text {máx }}, \quad y_{\text {mín }} \leqslant y \leqslant y_{\text {máx }}, \quad h(x, y) \leqslant z \leqslant z_{\text {máx }}\right\}
$$

donde $h(x, y)$ es la elevación del terreno sobre el punto $(x, y)$ en el plano tangente $x y$.

La velocidad del viento relativa al sistema $x y z$ está dada por

$$
\mathbf{v}_{\text {real }}=v_{\text {real }}^{i} \hat{\mathbf{x}}_{i}
$$

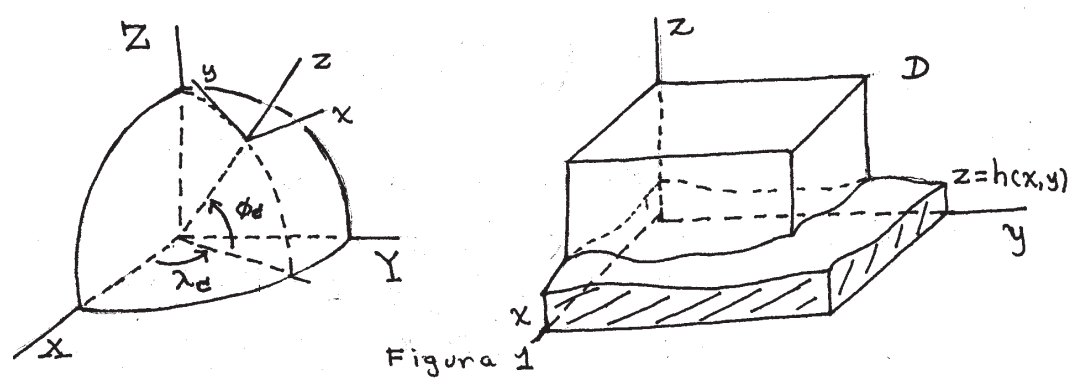

Figura 5.1: Sistema de referencia primario $X Y Z$ y sistema $x y z$. 
Para estimar el campo $\mathbf{v}_{\text {real }}$ suponemos (i) tiene lugar la ecuación de conservación de la masa en la forma

$$
\nabla \cdot \mathbf{v}_{\text {real }}=0, \quad\left(\nabla \equiv \hat{\mathbf{x}}_{i} \frac{\partial}{\partial x^{i}}\right)
$$

y (ii) el aire resbala en la superficie terrestre. Esta última condición tiene la forma

$$
\mathbf{v}_{\text {real }} \cdot \mathbf{n}=0 \quad \text { para } \quad z=h(x, y)
$$

donde $h(x, y)$ es la elevación del terreno sobre $(x, y)$ y $\mathbf{n}$ es un vector normal a la superficie terrestre,

$$
\mathbf{n}=\nabla(h-z)=\hat{\mathbf{x}} \partial_{x} h+\hat{\mathbf{y}} \partial_{y} h-\hat{\mathbf{z}} .
$$

Además, (iii) supondremos que tenemos un campo inicial

$$
\mathbf{v}^{0}=v^{i 0} \hat{\mathbf{x}}_{i}
$$

obtenido por interpolación de datos proporcionados por una red de monitoreo atmosférico. El postprocesamiento de datos considera: (i) la validación de datos, donde los valores fuera de lo esperado son eliminados y (ii) el reemplazamiento de datos incorrectos por medio de un método de interpolación [34]. En un contexto meteorológico estos pasos constituyen el llamado análisis objetivo espacial el cual considera el uso de esquemas de interpolación que, además, actúan como filtros [43], [8]. El resultado es un campo inicial de velocidades $\mathbf{v}^{0}(x)=v^{i 0}(x) \hat{\mathbf{x}}_{i}$ obtenido de un conjunto finito de datos $\left\{\mathbf{v}_{\text {true }}\left(x_{k}\right)\right\}_{k=1}^{N}$ medido en $\mathcal{D}$. Además de los esquemas meteorológicos para calcular $\mathbf{v}^{0}$ tenemos el método de correcciones sucesivas y la interpolación estadística [43], [8], la última es conocida como interpolación óptima o método de Gauss-Markov en las áreas de procesamiento de señales e imágenes [6].

Dado que, en general, el campo inicial $\mathbf{v}^{0}$ no satisface $\nabla \cdot \mathbf{v}^{0}=0$, el problema es estimar el campo $\mathbf{v}$ más cercano a $\mathbf{v}^{0}$ que satisface $\nabla \cdot \mathbf{v}=0$. Supongamos que $d(\mathbf{u}, \mathbf{v})$ (a lo que nos referiremos como métrica) proporciona una medida de la proximidad o distancia entre dos campos vectoriales $\mathbf{u}$ y $\mathbf{w}$. Entonces el problema para estimar el campo $\mathbf{v}_{\text {real }}$ por medio de un campo $\mathbf{v}$ puede enunciarse como sigue: Hallar el campo $\mathbf{v}$ más cercano a $\mathbf{v}^{0}$ con respecto a la métrica $d(\mathbf{u}, \mathbf{v})$, que satisfaga la ecuación

$$
\nabla \cdot \mathbf{v}=0
$$

y la condición de resbalamiento en la superficie terrestre,

$$
\mathbf{v} \cdot \mathbf{n}=0 \quad \text { para } \quad z=h(x, y) .
$$

Es claro que el campo estimado $\mathbf{v}$ puede no coincidir con el verdadero $\mathbf{v}_{\text {real }}$ dado que $\mathbf{v}$ se obtiene "ajustando" al campo inicial $\mathbf{v}^{0}$ por medio de la ecuación de continuidad, la condición de resbalamiento y una noción de distancia. Por tal motivo, nos referiremos a v como el campo ajustado. Para definir con mayor precisión el cálculo de $\mathbf{v}$ introduciremos algunos conceptos en la secciones siguientes.

\subsubsection{Normas vectoriales $\|\mathrm{w}\|_{S}$}

La forma más sencilla para obtener una noción de distancia entre vectores, es a través de una norma vectorial $[32]$

$$
\|\cdot\|: \mathbb{R}^{3} \rightarrow \mathbb{R}
$$

la cual asocia a cada vector $\mathbf{w}$ un número real no negativo $\|\mathbf{w}\|$ con las propiedades siguientes:

(i) $\|\mathbf{w}\| \geqslant 0 \mathrm{y}\|\mathbf{w}\|=0 \quad$ si y sólo si $\mathbf{w} \equiv \mathbf{0}$,

(ii) $\|\mathbf{w}+\mathbf{u}\| \leqslant\|\mathbf{w}\|+\|\mathbf{u}\|$,

(iii) $\|\alpha \mathbf{w}\|=|\alpha|\|\mathbf{w}\| \quad$ para cada escalar $\alpha \in \mathbb{R}$. Estas propiedades son una extensión de aquellas asociadas al valor absoluto $|x|$ de un número real $x$, si $|x-y|$ mide la distancia entre dos números reales $x$, $y$, para dos vectores $\mathbf{u}, \mathbf{v}$ definimos

$$
d(\mathbf{u}, \mathbf{w}) \equiv\|\mathbf{u}-\mathbf{w}\|
$$

como la métrica o distancia entre dichos vectores. Usando las propiedades de $\|\cdot\|$ es fácil ver que la función $d(\mathbf{u}, \mathbf{w})$ tiene las propiedades siguientes 
(i) $d(\mathbf{u}, \mathbf{w})=d(\mathbf{w}, \mathbf{u}) \quad$ (simetría),

(ii) $d(\mathbf{u}, \mathbf{w}) \geq 0$ y $d(\mathbf{u}, \mathbf{w})=0 \quad$ si y sólo si $\mathbf{u}=\mathbf{w}$,

(iii) $d(\mathbf{u}, \mathbf{v}) \leqslant d(\mathbf{u}, \mathbf{w})+d(\mathbf{v}, \mathbf{w})$ (la desigualdad triangular) .

La norma vectorial más común se obtiene a partir del producto escalar euclidiano

$$
\mathbf{w} \cdot \mathbf{u}=w^{i} \hat{\mathbf{x}}_{i} \cdot u^{j} \hat{\mathbf{x}}_{j}=w^{i} u^{i},
$$

y está dada por

$$
\|\mathbf{w}\|=\sqrt{\mathbf{w} \cdot \mathbf{w}} .
$$

Usando la desigualdad de Schwartz $|\mathbf{w} \cdot \mathbf{u}| \leqslant\|\mathbf{w}\|\|\mathbf{u}\|$ es fácil ver que $\|\cdot\|$ se satisface $\|\mathbf{w}+\mathbf{u}\| \leqslant\|\mathbf{w}\|+\|\mathbf{u}\|$.

Nota 5.1 El espacio $\mathbb{R}^{3}$ puede definirse como el conjunto de vectores columna

$$
\mathbf{w}=\left(\begin{array}{c}
w^{1} \\
w^{2} \\
w^{3}
\end{array}\right) .
$$

En particular, los vectores unitarios $\hat{\mathbf{x}}_{i}$ pueden definirse como

$$
\hat{\mathbf{x}}_{1}=\left(\begin{array}{c}
1 \\
0 \\
0
\end{array}\right) \quad \hat{\mathbf{x}}_{2}=\left(\begin{array}{l}
0 \\
1 \\
0
\end{array}\right) \quad \hat{\mathbf{x}}_{3}=\left(\begin{array}{l}
0 \\
0 \\
1
\end{array}\right) .
$$

Con la definición usual de multiplicación por un escalar y suma vectorial,

$$
\alpha \mathbf{u}+\beta \mathbf{w}=\left(\begin{array}{c}
\alpha u^{1} \\
\alpha u^{2} \\
\alpha u^{3}
\end{array}\right)+\left(\begin{array}{c}
\beta w^{1} \\
\beta w^{2} \\
\beta w^{3}
\end{array}\right),
$$

es inmediato que cada vector $\mathbf{w}$ tiene la forma

$$
\mathbf{w}=w^{i} \hat{\mathbf{x}}_{i}
$$

lo que da lugar a la identidad

$$
\mathbf{w}=\left(\begin{array}{c}
w^{1} \\
w^{2} \\
w^{3}
\end{array}\right)=w^{i} \hat{\mathbf{x}}_{i}
$$

La identificación de $\mathbf{w}$ como un vector columna permite expresar al producto interior euclidiano como un producto matricial. En efecto, el vector $\mathbf{w}$ es una matriz $3 \times 1$ (tres renglones y una columna) y el vector transpuesto $\mathbf{w}^{t}$ es el vector renglón o matriz $1 \times 3$

$$
\mathbf{w}^{t}=\left(\begin{array}{lll}
w^{1} & w^{2} & w^{3}
\end{array}\right) .
$$

De acuerdo con la multiplicación de matrices tenemos

$$
\mathbf{w}^{t} \mathbf{u}=\left(\begin{array}{ccc}
w^{1} & w^{2} & w^{3}
\end{array}\right)\left(\begin{array}{l}
u^{1} \\
u^{2} \\
u^{3}
\end{array}\right)=w^{i} u^{i} ;
$$

es decir,

$$
\mathbf{w} \cdot \mathbf{u}=\mathbf{w}^{t} \mathbf{u} .
$$

En general, para una matriz $\mathbb{S}_{3 \times 3}$ tenemos la identidad

$$
\mathbb{S}_{i j} w^{i} u^{j}=\mathbf{w}^{t} \mathbb{S} \mathbf{u}
$$

que usaremos para simplificar la notación en el presente trabajo. 
Con el propósito de dar un planteamiento general que nos permita analizar los distintos métodos usados en la literatura para calcular un campo ajustado v, consideraremos una norma más general definida a partir de una matriz simétrica $\mathbb{S}$ positiva definida. Decimos que $\mathbb{S}$ es simétrica positiva definida si tiene las propiedades siguientes:

(i) es simétrica $\left(\mathbb{S}=\mathbb{S}^{t}\right)$,

(ii) $\mathbf{w}^{t} \mathbb{S} \mathbf{w} \geq 0$ para cada vector $\mathbf{w} \in \mathbb{R}^{3}$ :,

(iii) $\mathbf{w}^{t} \mathbb{S} \mathbf{w}=0$ sólo para $\mathbf{w}=\mathbf{0}$.

Usando estas propiedades es fácil ver que

$$
\langle\mathbf{w} \mid \mathbf{u}\rangle_{S} \equiv \mathbb{S}_{i j} w^{i} u^{j}=\mathbf{w}^{t} \mathbb{S} \mathbf{u}
$$

define un producto interior, es decir, tiene las propiedades siguientes

(i) $<\mathbf{w}\left|\mathbf{u}>_{S}=<\mathbf{u}\right| \mathbf{w}>_{S}$ (simetría),

(ii) $<\mathbf{w}\left|\alpha \mathbf{u}+\beta \mathbf{v}>_{S}=\alpha<\mathbf{w}\right| \mathbf{u}>+\beta<\mathbf{w} \mid \mathbf{v}>\quad$ (linealidad),

(iii) $<\mathbf{w} \mid \mathbf{w}>\geqslant 0 \quad$ para cada $\mathbf{w}$,

(iv) $<\mathbf{w} \mid \mathbf{w}>=0 \quad$ sólo para $\mathbf{w}=\mathbf{0}$.

El número de normas vectoriales es infinito pero hay una relación entre ellas. Decimos que dos normas, vectoriales $\|\cdot\|_{a} \mathrm{y}\|\cdot\|_{b}$ con equivalentes si existen constantes $K_{1}, K_{2}>0$ tales que

$$
K_{1}\|\mathbf{w}\|_{a} \leqslant\|\mathbf{w}\|_{b} \leqslant K_{2}\|\mathbf{w}\|_{a} \quad \text { para cada } \mathbf{w} \in \mathbb{R}^{3} .
$$

Tenemos el resultado siguiente: Dos normas vectoriales cualesquiera $\|\cdot\|_{a} \mathrm{y}\|\cdot\|_{b}$ en $\mathbb{R}^{3}$, son equivalentes [15].

\subsubsection{Norma $\|\mathbf{w}\|_{\mathcal{D S}}$ de campos vectoriales $\mathbf{w}(x)$}

Hasta este momento hemos considerado la norma de un vector

$$
\mathbf{w}=w^{i} \hat{\mathbf{x}}_{i},
$$

cuyas componentes $w^{i}$ son números reales, pero en la estimación de un campo de vientos $\mathbf{v}(x)$ debemos tratar con campos vectoriales $\mathbf{w}(x)$ cuyas componentes dependen de cada punto en el espacio $x \equiv\left(x^{1}, x^{2}, x^{3}\right)$,

$$
\mathbf{w}=\mathbf{w}(x)=w^{i}(x) \hat{\mathbf{x}}_{i} .
$$

Así como la norma $\|\mathbf{w}\|$ de un vector $\mathbf{w}$ con componentes constantes $w^{i}$, se puede considerar como la extensión del valor absoluto $\left|w^{i}\right|$ de sus componentes, la norma de un campo vectorial $\mathbf{w}(x)$ se puede considerar como una extensión de la norma de una función $w^{i}(x)$. El producto interior entre funciones

$$
<f \mid g>=\int_{\mathcal{D}} f(x) g(x) d x
$$

define la norma para funciones

$$
\|f\|=<f \mid f>^{1 / 2} .
$$

El conjunto de funciones $f(x)$ cuya norma $\|f(x)\|$ es finita se denota con $L_{2}(\mathcal{D})$ y se conoce como el conjunto de funciones cuadrado integrables sobre la región $\mathcal{D}$,

$$
L_{2}(\mathcal{D})=\left\{f(x):\|f\|^{2}=\int_{\mathcal{D}}|f(x)|^{2} d x<\infty\right\}
$$

En nuestro caso las componentes $w^{i}(x)$ de un campo vectorial $\mathbf{w}(x)$ deben ser contínuas y cuadrado integrables en el conjunto acotado $\mathcal{D}$

$$
\int_{\mathcal{D}}\left|W^{k}(x)\right|^{2} d x<\infty
$$

Esto sugiere identificar a $\mathbf{w}(x)$ como un elemento del espacio vectorial $\mathbf{L}_{2}(\mathcal{D})$, constituido por los campos vectoriales cuyas componentes son cuadrado integrables,

$$
\mathbf{L}_{2}(\mathcal{D})=\left\{\mathbf{w}(x)=w^{i}(x) \hat{\mathbf{x}}_{i}: \int_{\mathcal{D}}\left|w^{i}(x)\right|^{2} d x<\infty\right\}
$$


El producto interior natural entre dos campos vectoriales $\mathbf{w}(x)$ y $\mathbf{u}(x)$ en $\mathbf{L}_{2}(\mathcal{D})$ se define por medio de la integración del producto interior euclidiano $\mathbf{w} \cdot \mathbf{u}=\mathbf{w}^{t} \mathbf{u}$

$$
<\mathbf{w} \mid \mathbf{u}>_{\mathcal{D}}=\int_{\mathcal{D}} \mathbf{w}^{t} \mathbf{u} d x .
$$

Donde $\langle\cdot \mid \cdot\rangle_{\mathcal{D}}$ tiene las propiedades de un producto interior

$$
\text { 1. }<\mathbf{w}\left|\mathbf{u}>_{\mathcal{D}}=<\mathbf{u}\right| \mathbf{w}>_{\mathcal{D}} \quad \text { simetría }
$$

2. $<\mathbf{w}\left|\alpha \mathbf{u}+\beta \mathbf{v}>_{\mathcal{D}}=\alpha<\mathbf{w}\right| \mathbf{u}>_{\mathcal{D}}+\beta<\mathbf{w} \mid \mathbf{v}>_{\mathcal{D}} \quad$ linealidad

3. $<\mathbf{w} \mid \mathbf{w}>_{\mathcal{D}} \geqslant 0$ y $\quad<\mathbf{w} \mid \mathbf{w}>_{\mathcal{D}}=0$ s y sólo sí $<\mathbf{w} \mid \mathbf{w}>_{\mathcal{D}}=0$.

La norma asociada $\mathrm{a}<\cdot|\cdot\rangle_{\mathcal{D}}$ es la integración de la norma vectorial $\|\cdot\|$,

$$
\|\mathbf{w}\|_{\mathcal{D}}^{2}=<\mathbf{w} \mid \mathbf{w}>_{\mathcal{D}}=\int_{\mathcal{D}}\|\mathbf{w}\|^{2} d x
$$

con lo cual obtenemos una medida de la distancia entre dos campos vectoriales

$$
\|\mathbf{w}-\mathbf{u}\|_{\mathcal{D}}^{2}=\int_{\mathcal{D}}\|\mathbf{w}-\mathbf{u}\|^{2} d x
$$

Extendamos lo anterior al caso del producto interior vectorial

$$
\langle\mathbf{w} \mid \mathbf{w}\rangle_{S}=\mathbf{w}^{t} \mathbb{S} \mathbf{u},
$$

definido por una matriz $\mathbb{S}$ simétrica positiva definida. Integrando obtenemos un producto interior entre dos campos vectoriales

$$
\langle\mathbf{w} \mid \mathbf{u}\rangle_{\mathcal{D} S} \equiv \int_{\mathcal{D}}\langle\mathbf{w} \mid \mathbf{u}\rangle_{S} d x=\int_{\mathcal{D}} \mathbf{w}^{t} \mathbb{S} \mathbf{u} d x .
$$

La norma asociada está dada por

$$
\|\mathbf{w}\|_{\mathcal{D} S}^{2}=\langle\mathbf{w} \mid \mathbf{w}\rangle_{\mathcal{D} S}=\int_{\mathcal{D}}\|\mathbf{w}\|_{S}^{2} d x
$$

y la métrica correspondiente es

$$
\|\mathbf{w}-\mathbf{u}\|_{\mathcal{D} S}
$$

Se puede demostrar que $\langle\cdot \mid \cdot\rangle_{\mathcal{D} S}$ satisface las propiedades de un producto interior,

$$
\begin{array}{ccll}
(\text { i }) & <\mathbf{w} \mid \mathbf{u}>_{\mathcal{D} S}= & <\mathbf{u} \mid \mathbf{w}>_{\mathcal{D} S} & \text { (simetría) } \\
(\text { ii }) & <\mathbf{w} \mid \alpha \mathbf{u}+\beta \mathbf{v}>_{\mathcal{D} S}= & \alpha<\mathbf{w}\left|\mathbf{u}>_{\mathcal{D} S}+\beta<\mathbf{w}\right| \mathbf{v}>_{\mathcal{D}} & \text { (linealidad) } \\
(\text { iii }) & <\mathbf{w} \mid \mathbf{w}>_{\mathcal{D} S} \geqslant & 0 \quad \mathrm{y}<\mathbf{w} \mid \mathbf{w}>_{\mathcal{D} S}=0 & \text { síy sólo sí } \mathbf{w}=\mathbf{0} .
\end{array}
$$

\subsection{Cálculo del campo ajustado $\mathrm{v}$ minimizando $\left\|\mathrm{w}-\mathrm{v}^{0}\right\|_{\mathcal{D S}}$ con $\nabla \cdot \mathbf{v}=0$}

En lo que sigue $\Gamma$ representará a la frontera de la región $\mathcal{D}$ y supondremos que tiene la descomposición

$$
\Gamma=\Gamma_{D} \cup \Gamma_{N}
$$

y $\mathbf{n}$ será un vector normal y exterior a la superficie $\Gamma$. El significado de los subíndices (Dirichlet y Neumann) quedará claro más adelante.

Sea $\mathbf{v}^{0}$ un campo obtenido a partir del procesamiento de un conjunto de datos $\left\{\mathbf{v}_{\text {true }}\left(x_{k}\right)\right\}_{k=1}^{N}$. En general $\mathbf{v}^{0}$ no satisface la ecuación de continuidad para flujos incompresibles por lo que el problema consiste en estimar un campo ajustado $\mathbf{v}$ cercano $\mathbf{v}^{0}$ (en el sentido definido abajo) que satisface

$$
\nabla \cdot \mathbf{v}=0 \text { en } \mathcal{D},
$$


además, el flujo es ideal y se mueve tangencialmente a la frontera sólida de $\mathcal{D}\left(\Gamma_{N}\right)$ por lo quesatisface la condición de frontera

$$
\mathbf{v} \cdot \mathbf{n}=0 \text { sobre } \Gamma_{N}
$$

Las condiciones (5.1) y (5.2) son suficientes para garantizar la existencia y unicidad de v. Si tenemos en mente que $\mathbf{v}$ es una pequeña modificación de $\mathbf{v}^{0}$ que satisface (5.1) entonces podemos afirmar que $\mathbf{v}$ es una aproximación aceptable aproximación de $\mathbf{v}_{\text {true }}$ en $\mathcal{D}$. Desde luego que la cercanía entre $\mathbf{v}$ y $\mathbf{v}_{\text {True }}$ está determinada principalmente por la cantidad de datos usados para determinar $\mathbf{v}^{0}$. Si tenemos información acerca de la capa de frontera física en $\Gamma_{N}$, ésta puede ser usada en la estimación de $\mathbf{v}^{0}$ como en varios Modelos de Masa Constante (MMC) [19].

El campo ajustado $\mathbf{v}$ se puede definir como un campo que minimiza el funcional cuadrático

$$
J(\mathbf{w})=\left\|\mathbf{w}-\mathbf{v}^{0}\right\|_{\mathcal{D} S}^{2}
$$

en el espacio de campos vectoriales w que satisfacen(5.1, 5.2). Para caracterizar tal espacio se utilizan algunos resultados publicados en $[9,18,42]$. El conjunto

$$
H(\operatorname{div})=\left\{\mathbf{w} \in \mathbf{L}^{2}(\overline{\mathcal{D}}): \nabla \cdot \mathbf{w} \in L^{2}(\overline{\mathcal{D}})\right\}
$$

es un espacio de Hilbert con el producto interior

$$
\langle\mathbf{w} \mid \mathbf{u}\rangle_{H(\text { div })}=\langle\mathbf{w} \mid \mathbf{u}\rangle_{\mathcal{D}}+\langle\nabla \cdot \mathbf{w} \mid \nabla \cdot \mathbf{u}\rangle
$$

en donde $\nabla \cdot \mathbf{w}$ se considera en el sentido débil en $L^{2}(\mathcal{D})$. Supondremos de aquí en adelante que el campo $\mathbf{v}^{0}$, definido como una interpolación de los datos de viento, pertenece al espacio $H(d i v)$. También, como parte de este espacio se encuentra el conjunto

$$
\mathbb{V}=\left\{\mathbf{w} \in H(\operatorname{div}): \nabla \cdot \mathbf{w}=0 \quad \text { en } \quad \mathcal{D},\left.\quad \mathbf{w} \cdot \mathbf{n}\right|_{\Gamma_{N}}=0\right\},
$$

el cual es un subespacio de $\mathbf{L}^{2}(\mathcal{D})[9,42]$, como el espacio que contiene a los posibles campos ajustados.

Nota 5.2 Para verificar la validez de la condición de frontera $\mathbf{w} \cdot \mathbf{n}=0$ sobre la frontera $\Gamma_{N}$ se define a $C_{0}^{\infty}(\overline{\mathcal{D}})=\left\{\left.\phi\right|_{\mathcal{D}}: \phi \in \mathcal{D}\left(\mathbb{R}^{3}\right)\right\}$ como el conjunto de funciones escalares infinitamente diferenciales con soporte compacto en $\overline{\mathcal{D}}$ y a $C_{0}^{\infty}(\overline{\mathcal{D}})^{3}$ como el espacio de los campos vectoriales con componente en $C_{0}^{\infty}(\overline{\mathcal{D}})$. Como puede verse en [9, p27], $C_{0}^{\infty}(\overline{\mathcal{D}})^{3}$ es denso en $H($ div $)$ y el mapeo $\gamma_{h}:\left.\mathbf{w} \rightarrow \mathbf{w} \cdot \mathbf{n}\right|_{\Gamma}$ puede extenderse a un mapeo lineal y continuo representado aún por $\gamma_{n}: H($ div $) \rightarrow H^{1 / 2}(\Gamma)$. Esto garantiza que la expresión $\mathbf{w} \cdot \mathbf{n}=0$ está bien definida en $\Gamma_{N}$ para cada $\mathbf{w} \in \mathbb{V} \subset H($ div $)$.

El conjunto

$$
\mathcal{V}=\left\{\phi \in \mathcal{D}^{3}: \nabla \cdot \phi=0 \quad \text { en } \quad \mathcal{D}\right\}
$$

es un subespacio de $\mathbb{V}$. Sea $H_{\mathcal{V}}$ la cerradura de $\mathcal{V}$ con la norma usual de $\mathbf{L}^{2}(\mathcal{D})$, y sea $H_{\mathcal{V}}$ el complemento ortogonal correspondiente. El teorema de proyección afirma que el espacio $\mathbf{L}^{2}$ tiene la descomposición

$$
\mathbf{L}^{2}=H_{\mathcal{V}} \oplus H_{\mathcal{V}}^{\perp}
$$

con el producto interior estándar $\langle\cdot \mid \cdot\rangle_{\mathcal{D}}$. De acuerdo con el teorema 2,7 de $[9]$

$$
H^{\perp}=\left\{\nabla q: q \in H^{1}(\mathcal{D})\right\}
$$

donde $H^{1}$ es el conjunto de funciones $\phi(x)$ con primeras derivadas $\partial \phi / \partial x^{i}$ en $L^{2}(\mathcal{D})$. Si $\overline{\mathbb{V}}$ es la cerradura de $\mathbb{V}$, visto como subespacio de $\mathbf{L}^{2}(\mathcal{D})$, entonces $\overline{\mathbb{V}}^{\perp}=\mathbb{V}^{\perp}$ y la relación $\mathcal{V} \subset \mathbb{V}$ implica $H_{\mathcal{V}}=\overline{\mathcal{V}} \subset \overline{\mathbb{V}}$ y $\mathbb{V} \perp \subset H_{\mathcal{V}}^{\perp}$ Así, para cada $\mathbf{u} \in \mathbb{V}^{\perp}$ existe una $q \in H^{1}(\mathcal{D})$ tal que $\mathbf{u}=\nabla q$ y $\langle\mathbf{w} \mid \nabla q\rangle_{\mathcal{D}}=0$ para cada $\mathbf{w} \in \mathbb{V}$. La identidad de Green

$$
\langle\mathbf{w} \mid \nabla q\rangle_{\mathcal{D}}+\langle\nabla \cdot \mathbf{w} \mid q\rangle_{\mathcal{D}}=\langle\mathbf{w} \cdot \mathbf{n} \mid q\rangle_{\Gamma}
$$

se cumple para toda $\mathbf{w} \in H\left(\right.$ div) y toda $q \in H^{1}(\mathcal{D})$, donde $\langle f \mid g\rangle_{\Gamma}=\int_{\Gamma} f g d s$ es el producto interior del espacio de Hilbert. En particular para $\mathbf{w} \in \overline{\mathbb{V}}$ y $\nabla q \in \mathbb{V}^{\perp}$ tenemos que $\langle\mathbf{w} \mid \nabla q\rangle_{\mathcal{D}}=\langle\nabla \cdot \mathbf{w} \mid q\rangle_{\mathcal{D}}=0$, entonces

$$
\langle\mathbf{w} \cdot \mathbf{n} \mid q\rangle_{\Gamma_{D}}=0 .
$$


De aquí se concluye que $q$ debe satisfacer la siguiente condición de frontera:

$$
q=0 \text { sobre } \Gamma_{D}
$$

En términos del conjunto

$$
H_{\mathcal{D}}^{1}=\left\{\phi \in H^{1}(\mathcal{D}): \phi=0 \quad \text { en } \quad \Gamma_{\mathcal{D}}\right\}
$$

podemos escribir

$$
\mathbb{V}^{\perp}=\left\{\nabla q: q \in H_{\mathcal{D}}^{1}(\mathcal{D}) .\right\}
$$

Para demostrar la existencia y unicidad del campo ajustado usando el teorema de proyección, sea $\mathbb{V}_{S}$ la cerradura de $\mathbb{V}$ con respecto a la norma $\|\cdot\|_{\mathcal{D} S}$. Dado que las normas $\|\cdot\|_{\mathcal{D}}$ y $\|\cdot\|_{\mathcal{D} S}$ son equivalentes, tenemos $\overline{\mathbb{V}}=\mathbb{V}_{S}$. Entonces el conjunto

$$
\mathbb{V}_{S}^{\perp}=\left\{\mathbb{S}^{-1} \nabla q: q \in H_{\mathcal{D}}^{1}(\mathcal{D})\right\}
$$

es el complemento ortogonal de $\mathbb{V}_{S}$ en $\mathbb{L}^{2}(\mathcal{D})$ con el producto interior $\langle\cdot \mid \cdot\rangle_{\mathcal{D} S}$. En efecto, sea $\mathbf{u} \in \mathbb{V} \frac{1}{S}$ y $\mathbf{w} \in \mathbb{V}_{S}$, entonces

$$
\langle\mathbf{w} \mid \mathbf{u}\rangle_{\mathcal{D} S}=\langle\mathbf{w} \mid \mathbb{S u}\rangle_{\mathcal{D}}=\left\langle\mathbf{w} \mid \mathbb{S S}^{-1} \nabla q\right\rangle_{\mathcal{D}}=\langle\mathbf{w} \mid \nabla q\rangle_{\mathcal{D}}=0
$$

Con esto se ve que, efectivamente, los espacios $\mathbb{V}$ y $\mathbb{V} \frac{\perp}{S}$ son ortogonales en la norma $\|\cdot\|_{\mathcal{D} S}$, en consecuencia se tiene la descomposición

$$
\mathbf{L}^{2}(\mathcal{D})=\mathbb{V} \oplus \mathbb{V} \stackrel{\perp}{S}
$$

De acuerdo con el Teorema de Proyección, para cada $\mathbf{v}^{0} \in H(\operatorname{div}) \subset \mathbf{L}^{2}$, existe un único $\mathbf{v} \in \mathbb{V}$ tal que

$$
\left\|\mathbf{v}-\mathbf{v}^{0}\right\|_{\mathcal{D} S}^{2}=\operatorname{mín}_{\mathbf{w} \in \mathbb{V}_{S}}\left\|\mathbf{w}-\mathbf{v}^{0}\right\|_{\mathcal{D} S}^{2}=\operatorname{mín}_{\mathbf{w} \in \mathbb{V}_{S}} J(\mathbf{w})
$$

y

$$
\mathbf{v}^{0}=\mathbf{v}-\mathbf{u}
$$

con $\mathbf{u} \in \mathbb{V} \frac{\perp}{S}$. Esto demuetra la existencia y unicidad del campo ajustado v. La caracterización de $\mathbb{V} \frac{\perp}{S}$ implica que existe $\lambda \in H_{\mathcal{D}}^{1}(\mathcal{D})$ tal que $\mathbf{u}=\mathbb{S}^{-1} \nabla \lambda$ con lo cual podemos escribir

$$
\mathbf{v}=\mathbf{v}^{0}+\mathbb{S}^{-1} \nabla \lambda
$$

Las primeras derivadas $\partial \lambda / \partial x^{i}$ pertenecen a $L^{2}(\mathcal{D})$ pero el lado derecho de $(5.6)$ no puede ponerse en (5.1) para obtener una ecuación para $\lambda$ ya que la ecuación resultante

$$
\nabla \cdot \mathbf{v}=\nabla \cdot \mathbf{v}^{0}+\nabla \cdot \mathbb{S}^{-1} \nabla \lambda=0
$$

sólo tiene sentido si $\lambda$ tiene segundas derivadas $\partial^{2} \lambda / \partial x^{i} \partial x^{j}$ en $L^{2}(\mathcal{D})$. Podemos evitar este problema reescribiendo (5.1) en una forma débil.

De acuerdo con (5.5), si v pertenece a $\mathbb{V} \subset H($ div), debe satisfacer

$$
\langle\mathbf{v} \mid \nabla \phi\rangle_{\mathcal{D}}=0 \quad \forall \quad \phi \in H_{D}^{1}(\mathcal{D}) \text {. }
$$

Esta es la forma débil deseada de la ecuación (5.1,5.2). La sustitución de (5.6) en (5.7) implica que $\lambda$ debe satisfacer la ecuación integral

$$
a(\lambda, \phi)=-\left\langle\mathbf{v}^{0} \mid \nabla \phi\right\rangle_{\mathcal{D}} \quad \forall \quad \phi \in H_{D}^{1}(\mathcal{D})
$$

donde establecemos

$$
a(\lambda, \phi) \equiv\left\langle\mathbb{S}^{-1} \nabla \lambda \mid \nabla \phi\right\rangle_{\mathcal{D}}=\left\langle\mathbb{S}_{j i}^{-1} \frac{\partial \lambda}{\partial x^{i}} \mid \frac{\partial \phi}{\partial x^{j}}\right\rangle .
$$

Entonces la minimización de $J(\cdot)$ es reemplazada por la solución de (5.8).

Para demostrar la existencia y unicidad de la solución $\lambda$ de la ecuación (5.8) usemos (5.5) para reescribir el lado derecho de la ecuación (5.8) y obtenemos el problema

$$
a(\lambda, \phi)=\left\langle\nabla \cdot \mathbf{v}^{0} \mid \phi\right\rangle_{\mathcal{D}}+\left\langle-\mathbf{v}^{0} \cdot \mathbf{n} \mid \phi\right\rangle_{\Gamma_{N}}
$$

para toda $\phi \in H_{D}^{1}(\mathcal{D})$. Ahora se toman en cuenta los siguientes hechos: 
- El conjunto $H_{D}^{1}(\mathcal{D})$ es un espacio de Hilbert con el producto interior

$$
\langle\psi \mid \phi\rangle_{1}=\langle\psi \mid \phi\rangle+\langle\nabla \psi \mid \nabla \phi\rangle \quad \forall \psi, \phi \in H_{D}^{1}(\mathcal{D})
$$

y la correspondiente norma $\|\cdot\|_{1}$;

- Si $\mathbb{S}(x)$ es simétrica y positiva definida en $\bar{D}$, su inversa $\mathbb{S}^{-1}(x)$ también lo es. Esto implica que la forma bilineal $a(\cdot, \cdot)$ es simétrica $[a(\psi, \phi)=a(\phi, \psi)]$ y coerciva $\left(H_{D}^{1}\right.$-elíptica $)$, i.e., existe una contante positiva $c_{D}$ tal que

$$
a(\phi, \phi) \geq c_{D}\|\phi\|_{1}^{2} \quad \forall \phi \in H_{D}^{1}(\mathcal{D})
$$

- Si cada elemento $\mathbb{S}_{i j}^{-1}(x)$ pertenece a $C^{(0)}(\bar{D})$, la forma $a(\cdot, \cdot)$ es acotada en $H_{D}^{1}(\mathcal{D})$,

$$
a(\phi, \psi) \leq K \quad\|\phi\|_{1} \quad\|\psi\|_{1} \quad \forall \psi, \phi \in H_{D}^{1}(\mathcal{D})
$$

Estas propiedades junto con $\mathbf{v}^{0} \in H($ div) garantizan, por el teorema de Lax-Milgram que existe una única solución $\lambda$ de la ec. (5.10) en $H_{D}^{1}(\mathcal{D})$, que satisface la CFD

$$
\lambda=0 \text { en } \Gamma_{D} .
$$

De estos resultados se deriva la siguiente proposición.

Proposición 5.1 Sean $\mathbb{S}(x)$ una matriz definida positiva en $\overline{\mathcal{D}}, \mathbf{v}^{0} \in H($ div $)$ y $\mathbb{S}_{i j}^{-1}(x) \in C^{(0)}(\bar{D})$. Entonces existe una única $\lambda$ en $H_{D}^{1}(\mathcal{D})$ que satisface (5.8).

Bajo condiciones generales , $\lambda$ proporciona un campo ajustado v (5.6) que satisface la ecuación de continuidad en su forma fuerte (5.1) y la condición de frontera (5.2). Sea $H^{2}(\mathcal{D})$ El espacio de Sobolev de funciones con segundas derivadas en $L^{2}(\mathcal{D})$, y $C^{(1)}(\bar{D})$ las funciones con primeras derivadas continuas en $\bar{D}$. Las condiciones son dadas básicamente por

Teorema 5.1 [36, p.548]. Si $\nabla \cdot \mathbf{v}^{0} \in L^{2}(\mathcal{D})$ y $\mathbb{S}_{i j}^{-1}(x) \in C^{(1)}(\bar{D})$, la solución $\lambda$ de (5.8) pertenece a $H^{2}\left(\mathcal{D}^{\prime}\right)$ donde $\mathcal{D}^{\prime}$ es una región abierta tal que $\bar{D}^{\prime} \subset \mathcal{D}$.

Nota 5.3 La forma más común de $\mathbb{S}$ en la literatura meteorológica [35] es $\mathbb{S}_{i j}=\alpha_{i}^{2} \delta_{i j}$ con $\alpha_{i}$ constantes En este caso $\mathbb{S}_{i j}^{-1} \in C^{(\infty)}(\mathcal{D})$ (el conjunto de funciones infinitamente diferenciables en $\mathcal{D}$ ) y los esquemas de interpolación comunes [35] proporcionan un campo $\mathbf{v}^{0}$ con componentes en $C^{(\infty)}(\mathcal{D})$, tal que $\lambda \in C^{(\infty)}(\mathcal{D})$ y $\mathbf{v} \in C^{(\infty)}(\mathcal{D})^{3}$. Por lo que, en adelante consideraremos que los datos del problema (5.8) son suaves cuando $\lambda$ pertenezca a $H^{2}(\mathcal{D})$. Los dominios $\mathcal{D}$ usuales tienen esquinas donde $\lambda$ pierde regularidad $y$, en consecuencia, es posible que $\mathbf{v}$ no satisfaga $\nabla \cdot \mathbf{v}=0$ en $\Gamma$ (ver, e.g., [36, cap.46], [17, p.18]).

Sea $\mathbf{v}^{0}, \mathbb{S}_{i j}^{-1}$ definida como en el Teorema 5.1 y sea $\lambda \in H^{2}(\mathcal{D})$. Entonces podemos usar la fórmula de Green

$$
\int_{\mathcal{D}} u \frac{\partial v}{\partial x^{j}} d x+\int_{\mathcal{D}} \frac{\partial u}{\partial x^{j}} v d x=\int_{\Gamma} u v n_{j} d s
$$

con $u=\mathbb{S}_{j i}^{-1} \partial \lambda / \partial x^{i}$ y $v=\phi$ para integrar $a(\cdot, \cdot)(5.9)$ y obtener

$$
a(\lambda, \phi)=\langle L \lambda \mid \phi\rangle+\langle\phi \mid \mathcal{L} \lambda\rangle_{\Gamma_{N}} \forall \phi \in H_{D}^{1}(\mathcal{D}),
$$

donde $L$ es el operador elíptico

y $\mathcal{L}$ es la derivada co-normal

$$
L=-\nabla^{t} \mathbb{S}^{-1} \nabla=-\frac{\partial}{\partial x^{j}} \mathbb{S}_{j i}^{-1} \frac{\partial}{\partial x^{i}}
$$

$$
\mathcal{L} \lambda \equiv \mathbf{n} \cdot \mathbb{S}^{-1} \nabla \lambda=n_{j} \mathbb{S}_{j i}^{-1} \frac{\partial \lambda}{\partial x^{i}} .
$$

Por lo tanto, la ecuación (5.10) toma la forma

$$
\langle L \lambda \mid \phi\rangle=\left\langle\nabla \cdot \mathbf{v}^{0} \mid \phi\right\rangle-\left\langle\mathbf{v}^{0} \cdot \mathbf{n}+\mathcal{L} \lambda \mid \phi\right\rangle_{\Gamma_{N}}
$$


para toda $\phi \in H_{D}^{1}(\mathcal{D})$. En particular para $\phi \in C_{0}^{\infty}(\mathcal{D}) \subset H_{D}^{1}(\mathcal{D})$ tenemos $\left.\phi\right|_{\Gamma}=0 \mathrm{y}$

$$
\langle L \lambda \mid \phi\rangle=\left\langle\nabla \cdot \mathbf{v}^{0} \mid \phi\right\rangle \forall \phi \in C_{0}^{\infty}(\mathcal{D}) .
$$

Ya que $C_{0}^{\infty}(\mathcal{D})$ es denso en $L^{2}(\mathcal{D})$, se sigue que $\lambda$ es una solución fuerte de la ecuación elíptica

$$
L \lambda=\nabla \cdot \mathbf{v}^{0} \text { en } \mathcal{D}
$$

y (5.16) toma la forma

$$
\left\langle\mathbf{v}^{0} \cdot \mathbf{n}+\mathcal{L} \lambda \mid \phi\right\rangle_{\Gamma_{N}}=0 \quad \forall \phi \in H_{D}^{1}(\mathcal{D}) .
$$

Esto significa que $\lambda$ tiene la propiedad de satisfacer las Condiciones de Frontera Neumann (CFN)

$$
\mathbf{v}^{0} \cdot \mathbf{n}+\mathcal{L} \lambda=0 \text { sobre } \Gamma_{N}
$$

y, En consecuencia, el campo ajustado v (5.6) satisface (5.2).

Proposición 5.2 Sean $\mathbb{S}, \mathbf{v}^{0}, \mathbb{S}^{-1}$ definidas como en las proposiciones ?? y 5.1 y supongamos que los datos del problema (5.8) son suaves de tal forma que $\lambda \in H^{2}(\mathcal{D})$. Entonces $\lambda$ satisface (5.17) y las condiciones de frontera (5.11,5.18). El campo $\mathbf{v}=\mathbf{v}^{0}+\mathbb{S}^{-1} \nabla \lambda$ satisface $(5.1,5.2)$ y minimiza globalmente a $J(\cdot)$ en $\mathbb{V}$.

\subsection{Formulación variacional del problema elíptico generalizado $L_{y} \lambda=f$}

En esta sección consideramos la formulación variacional del problema elíptico (5.17) en coordenadas arbitrarias con el propósito de dar un planteamiento que incluya tanto la formulación en coordenadas cartesianas $x y z$ como en coordenadas generalizadas $y^{1} y^{2} y^{3}$. El planteamiento en coordenadas cartesianas se recupera de lo expuesto en esta sección haciendo $\mathbb{M}=\mathbb{S}, M=S, \sqrt{g}=1$ y eliminando el subíndice $y$. La región $\mathcal{D}_{y}$ es la imagen de $\mathcal{D}$ bajo una transformación de coordenadas $x^{i}=x^{i}\left(y^{1}, y^{2}, y^{3}\right)$, en forma análoga definimos $\Gamma_{D y}$, $\Gamma_{N y}$.

$$
L_{y} \lambda=f \quad \text { en } \quad \mathcal{D}_{y}
$$

con las condiciones de frontera mixtas

$$
\begin{array}{rlll}
\lambda=0 & \text { en } & \Gamma_{D y}, \\
\mathcal{L}_{y} \lambda=q & \text { en } & \Gamma_{N y},
\end{array}
$$

donde $\Gamma_{y}=\Gamma_{D y} \cup \Gamma_{N y}$ es la frontera de la región $\mathcal{D}_{y}$ en el espacio $y$, y $L_{y}, \mathcal{L}_{y}, f, q$ están dados por

$$
L_{y}=-\nabla^{t} \sqrt{g} \mathbb{M}^{-1} \nabla \quad f=\sqrt{g} \nabla \cdot \mathbf{v}^{0} \quad \mathcal{L}_{y}=\hat{\mathbf{n}}_{y}^{t} \mathbb{M}^{-1} \nabla \quad q=-\hat{\mathbf{n}}_{y}^{t} \mathbf{v}^{0} .
$$

La solución $\lambda$ es (al menos) de cuadrado integrable por lo que pertenece al espacio

$$
L_{2}\left(\mathcal{D}_{y}\right)=\left\{v: \int_{\mathcal{D}_{y}}|v|^{2} d y<\infty\right\}
$$

dotado con el producto interior

$$
\langle v \mid u\rangle_{y} \equiv \int_{\mathcal{D}_{y}} v u d y,
$$

y la norma $\|v\|_{y}=\langle v \mid v\rangle_{y}^{1 / 2}$. Además, $\lambda$ debe pertenecer al espacio de funciones que satisfacen la condiciones de frontera Dirichlet (5.20), espacio que denotamos por $V_{a}$. En lo que sigue consideramos que $V_{a}$ es completo con la norma

$$
\|v\|_{y 1}^{2} \equiv\|v\|_{y}^{2}+\sum_{k}\left\|\frac{\partial v}{\partial y^{k}}\right\|_{y}^{2} ;
$$


es decir,

$$
V_{a}=\left\{v \in H^{1}\left(\mathcal{D}_{y}\right): v=0 \text { sobre } \Gamma_{y}\right\} .
$$

Para obtener la formulación variacional (o débil) del problema elíptico (5.19-5.21) multiplicamos (5.19) por una función $v$ en $V_{a}$ e integramos,

$$
\left\langle L_{y} \lambda \mid v\right\rangle_{y}=\langle f \mid v\rangle_{y} \quad \text { para cada } v \in V_{a}
$$

El lado izquierdo se integra usando el teorema de Green en el espacio $y$

$$
\int_{\mathcal{D} y} \frac{\partial w}{\partial y^{k}} v d y=\oint_{\Gamma y} v w \hat{n}_{y k} d s_{y}-\int_{\mathcal{D} y} w \frac{\partial v}{\partial y^{k}} d y
$$

donde $\hat{n}_{y k}$ son las componentes del vector normal, unitario y exterior a $\Gamma_{y}$. Haciendo cálculos se obtiene

$$
\begin{aligned}
\left\langle L_{y} \lambda \mid v\right\rangle_{y} & =-\sum_{k l}\left\langle\frac{\partial}{\partial y^{k}} \sqrt{g} M_{k l}^{-1} \frac{\partial \lambda}{\partial y^{l}} \mid v\right\rangle_{y} \\
& =-\sum_{k l}\left\{\oint_{\Gamma_{y}} v \sqrt{g} M_{k l}^{-1} \frac{\partial \lambda}{\partial y^{l}} \hat{n}_{y k} d s_{y}-\int_{\mathcal{D}_{y}} \sqrt{g} M_{k l}^{-1} \frac{\partial \lambda}{\partial y^{l}} \frac{\partial v}{\partial y^{k}} d y\right\} \\
& =-\oint_{\Gamma_{y}} \sqrt{g} v\left(\sum_{k l} \hat{n}_{y k} M_{k l}^{-1} \frac{\partial}{\partial y^{l}}\right) \lambda d s_{y}+\sum_{k l}\left\langle\sqrt{g} M_{k l}^{-1} \frac{\partial \lambda}{\partial y^{l}} \mid \frac{\partial v}{\partial y^{k}}\right\rangle_{y} \\
& =-\oint_{\partial \Gamma_{y}} \sqrt{g} v \mathcal{L}_{y} \lambda d s_{y}+a_{y}(\lambda, v),
\end{aligned}
$$

donde aparece el operador

$$
\mathcal{L}_{y}=\sum_{k l} \hat{n}_{y k} M_{k l}^{-1} \frac{\partial}{\partial y^{l}}
$$

y la forma bilineal

$$
a_{y}(u, v) \equiv \sum_{k l}\left\langle\sqrt{g} M_{k l}^{-1} \frac{\partial u}{\partial y^{l}}, \frac{\partial v}{\partial y^{k}}\right\rangle_{y}=\int_{\mathcal{D}_{y}}(\nabla u)^{t} \mathbb{M}^{-1} \nabla v \sqrt{g} d y
$$

Entonces (5.22) queda como sigue

$$
a_{y}(\lambda, v)=\langle f \mid v\rangle_{y}+\oint_{\Gamma_{y}} \sqrt{g} v \mathcal{L}_{y} \lambda d s_{y} \quad \forall v \in V_{a} .
$$

Dado que $v$ satisface $v=0$ en $\Gamma_{y}$ y $\mathcal{L}_{y} \lambda=q$ tiene lugar en $\Gamma_{N y}$, la integral de superficie toma la forma

$$
\oint_{\Gamma_{y}} \sqrt{g} v \mathcal{L}_{y} \lambda d s_{y}=\int_{\Gamma_{N y}} \sqrt{g} v q d s_{y} .
$$

Así, concluimos que si $\lambda$ existe debe satisfacer la ecuación integral

$$
a_{y}(\lambda, v)=\tilde{f}_{y}(v) \quad \forall v \in V_{a}
$$

donde definimos

$$
\tilde{f}_{y}(v) \equiv\langle f \mid v\rangle_{y}+\int_{\Gamma_{N y}} \sqrt{g} v q d s_{y}
$$

La ecuación (5.23) recibe en nombre de forma débil del problema elíptico (5.19-5.21). La existencia y unicidad de la solución de la ecuación integral (5.23) está garantizada por las propiedades de $a_{y}(\cdot, \cdot)$ y $\tilde{f}_{y}(\cdot)$. Es fácil ver que $a_{y}(\cdot, \cdot)$ define un producto interior en el espacio $V_{a}$ dado que es:

1. simétrica, $a_{y}(u, v)=a_{y}(v, u)$, 
2. positiva definida, $a_{y}(u, u) \geq 0, \mathrm{y} a_{y}(u, u)=0$ sólo para $u=0$,

3. bilineal, $a_{y}\left(u, c_{1} v+c_{2} w\right)=c_{1} a_{y}(u, v)+c_{2} a_{y}(u, w)$.

En efecto, usando la simetría de $\mathbb{M}^{-1}$ se obtiene

$$
(\nabla u)^{t} \mathbb{M}^{-1} \nabla v=\left(\nabla u^{t} \mathbb{M}^{-1} \nabla v\right)^{t}=(\nabla v)^{t}\left(\mathbb{M}^{-1}\right)^{t} \nabla u=(\nabla v)^{t} \mathbb{M}^{-1} \nabla u,
$$

y por tanto

$$
a_{y}(u, v)=\int_{\mathcal{D}_{y}}(\nabla u)^{t} \mathbb{M}^{-1} \nabla v \sqrt{g} d y=\int_{\mathcal{D}_{y}}(\nabla v)^{t} \mathbb{M}^{-1} \nabla u \sqrt{g} d y=a_{y}(v, u)
$$

Si la transformación de coordenadas $x^{i}=x^{i}(y)$ es bien comportada y los coeficientes $\alpha_{i}^{2}(y)$ no se anulan en $\bar{D} \equiv \mathcal{D} \cup \Gamma$, entonces $\mathbb{M}^{-1}(y)$ es positiva definida en cada punto de la región $\bar{D}$, es decir,

$$
\mathbf{u}^{t} \mathbb{M}^{-1} \mathbf{u}>0 \text { para } \mathbf{u} \neq \mathbf{0} .
$$

En particular para $\mathbf{u}=\nabla u$ tenemos $(\nabla u)^{t} \mathbb{M}^{-1} \nabla u>0$ si $u$ no es una función constante, multiplicando la última desigualdad por $\sqrt{g}$ e integrando se concluye que $a_{y}(\cdot, \cdot)$ es positiva definida en $V_{a} ;$ es decir, $a_{y}(\cdot, \cdot)$ satisface

$$
a_{y}(u, u)>0 \forall u \in V_{a} .
$$

La linealidad de $a_{y}(\cdot, \cdot)$ es obvia. Finalmente si $f(y)$ y $q(y)$ son funciones continuas a trozos, el funcional $\tilde{f}_{y}(\cdot)$ es acotado. De acuerdo con el teorema de Lax-Milgram [36] estas propiedades garantizan la existencia y unicidad de la solución $\lambda$ de la ecuación integral $(5.23)$. La simetría de $a_{y}(\cdot, \cdot)$ permite identificar a las soluciones de la ecuación integral (5.23) como las extremales del funcional cuadrático

$$
J_{y}(v)=\frac{1}{2} a_{y}(v, v)-\tilde{f}_{y}(v) \quad \text { para } v \in V_{a} .
$$

Una función $u$ se llama extremal del funcional $J_{y}(v)$ si satisface la condición de extremo

$$
\left.\frac{d}{d \epsilon} J_{y}(u+\epsilon v)\right|_{\epsilon=0}=0 \forall v \in V_{a}
$$

De acuerdo con esta definición para mostrar que las soluciones de (5.23) son extremales de $J_{y}(v)$ basta con probar que (5.23) coincide con la condición de extremo. Usando la simetría y linealidad de $a_{y}(\cdot, \cdot)$ y $\tilde{f}_{y}(\cdot)$ se obtiene

$$
\begin{aligned}
J_{y}(\lambda+\epsilon v) & =\frac{1}{2} a_{y}(\lambda+\epsilon v, \lambda+\epsilon v)-\tilde{f}_{y}(\lambda+\epsilon v) \\
& =\frac{1}{2} a_{y}(\lambda, \lambda)+\epsilon a_{y}(\lambda, v)+\frac{1}{2} \epsilon^{2} a_{y}(v, v)-\tilde{f}_{y}(\lambda)-\epsilon \tilde{f}_{y}(v)
\end{aligned}
$$

Derivando y evaluando en $\epsilon=0$ se obtiene

$$
\left.\frac{d}{d \epsilon} J_{y}(\lambda+\epsilon v)\right|_{\epsilon=0}=a_{y}(\lambda, v)-\tilde{f}_{y}(v)=0
$$

que es exactamente la ecuación (5.23) y por consiguiente una solución $\lambda$ de (5.23) es una extremal de $J_{y}(v)$. El teorema de Lax-Milgram garantiza que existe una extremal $\lambda$ y es única.

De acuerdo con lo anterior, en lugar de calcular a $\lambda$ por medio de la ecuación integral (5.23), podemos obtenerla como la función que minimiza al funcional $J(\lambda)$. Este último camino tiene la ventaja de incorporar la condición de frontera Neumann (5.21) en $J(\lambda)$ por lo que sólo debemos considerar en forma explícita la condición de frontera Dirichlet (5.19). Para probar esto mostremos que si $\lambda$ es una extremal de $J(\lambda)$, entonces satisface la condición de frontera Neumann (5.21).

Arriba probamos que si $\lambda$ minimiza a $J_{y}(\lambda)$ satisface (5.23). Consideremos (5.23) con $v$ en $C_{0}^{\infty}\left(\mathcal{D}_{y}\right)$. Dado que cada $v$ se anula en toda la frontera $\Gamma_{y}, v$ pertenece al espacio $V_{a}$ por lo que $\lambda$ satisface (5.23) para cada $v \in C_{0}^{\infty}\left(\mathcal{D}_{y}\right)$

$$
a_{y}(\lambda, v)=\langle f \mid v\rangle_{y}+\oint_{\Gamma_{N y}} \sqrt{g} v q d s_{y} \quad \forall v \in C_{0}^{\infty}\left(\mathcal{D}_{y}\right),
$$


pero $v=0$ sobre $\Gamma_{y}$ lo que reduce la ecuación anterior a

$$
a_{y}(\lambda, v)=\langle f \mid v\rangle_{y} \quad \text { para } v \in C_{0}^{\infty}\left(\mathcal{D}_{y}\right)
$$

Por otro lado, si $\lambda$ tiene derivadas de segundo orden podemos integrar por partes el lado izquierdo para obtener

$$
a_{y}(\lambda, v)=\left\langle L_{y} \lambda \mid v\right\rangle_{y} \quad \text { para } v \in C_{0}^{\infty}\left(\mathcal{D}_{y}\right) .
$$

Reemplazando en la ecuación anterior se obtiene

$$
\left\langle L_{y} \lambda-f \mid v\right\rangle_{y}=0 \quad \text { para } v \in C_{0}^{\infty}\left(\mathcal{D}_{y}\right),
$$

y por tanto $\lambda$ satisface la ecuación elíptica

$$
L_{y} \lambda=f \quad \text { en } \mathcal{D}_{y}
$$

Si ahora consideramos la ecuación (5.23) en la forma

$$
\left\langle L_{y} \lambda \mid v\right\rangle_{y}+\int_{\Gamma_{N y}} \sqrt{g} v \mathcal{L}_{y} \lambda d s_{y}=\langle f \mid v\rangle_{y}+\int_{\Gamma_{N y}} \sqrt{g} v q d s_{y}
$$

y usamos el hecho que $\lambda$ satisface (5.24), los términos $\langle L \lambda \mid v\rangle_{y}$ y $\langle f \mid v\rangle_{y}$ se cancelan por lo que $\lambda$ satisface

$$
\int_{\Gamma_{N y}} \sqrt{g} v \mathcal{L}_{y} \lambda d s_{y}=\int_{\Gamma_{N y}} \sqrt{g} v q d s_{y} \quad \forall v \in V_{a}
$$

y dado que en general $v \neq 0$ se concluye que $\lambda$ debe satisfacer

$$
\mathcal{L}_{y} \lambda=q \quad \text { sobre } \Gamma_{N y} .
$$

En otras palabras, si $\lambda$ minimiza al funcional $J_{y}(v)$ entonces satisface la condición de frontera Neumann $(5.21)$.

\subsection{Cálculo de $\lambda$ con el método del elemento finito}

En esta sección consideramos la solución del problema con condiciones de frontera $(5.11,5.17,5.18)$ utilizando el método de Elemento finito (EF). Los MMC usan esquemas de diferencias para calcular $\lambda$ [35] pero en este trabajo se usa el EF ya que tiene la ventaja de la forma débil de la ecuación (5.17). De hecho, vemos que $\lambda$ es la única solución en $H_{D}^{1}(\mathcal{D})$ de la ecuación integral $(5.8)$

$$
\int_{\mathcal{D}}\left(\mathbb{S}^{-1} \nabla \lambda\right) \cdot \nabla \phi d x=-\int_{\mathcal{D}} \mathbf{v}^{0} \cdot \nabla \phi d x \text { para } \phi \in H_{D}^{1}(\mathcal{D}) .
$$

Esta ecuación sólo requiere de las CFD (5.11) por medio de las funciones $\phi$ ya que la CFN (5.18) es una propiedad intrínseca de $\lambda$. El EF introduce una aproximación

$$
\lambda_{h}(x)=\sum_{k=1}^{N_{0}} \lambda_{k} \phi_{k}(x)
$$

donde las funciones $\phi_{i}(x)$ son la base de un espacio de aproximación (definido abajo) de $H_{D}^{1}(\mathcal{D})$. En lo que sigue consideramos a la matriz $\mathbb{S}$ en su forma más común $\mathbb{S}_{i j}=\delta_{i j} \alpha_{i}^{2}[2]$, donde los elementos $\alpha_{i}$ son constantes. En este caso el campo ajustado v (5.6) está dado por

$$
v^{i}=v^{0, i}+\frac{1}{\alpha_{i}^{2}} \frac{\partial \lambda}{\partial x^{i}} .
$$


Sea $h$ el paso de discretización y sea $\tau_{h}$ un elemento finito de interpolación de $\mathcal{D}$, sea $P_{1}$ el espacio de polinomios de dos variables de grado $\leq 1$ y sea $C^{0}(\mathcal{D})$ el espacio de funciones continuas en $\bar{D}$. Los espacios $H^{1}(\mathcal{D})$ y $H_{D}^{1}(\mathcal{D})$ son aproximados por los espacios de dimensión finita

$$
\begin{aligned}
W_{h} & =\left\{\phi_{h} \in C^{0}(\mathcal{D}):\left.\phi_{h}\right|_{T} \in P_{1} \text { para } T \in \tau_{h}\right\}, \\
W_{0 h} & =\left\{\phi_{h} \in W_{h}: \phi_{h}=0 \text { en } \Gamma_{D}\right\},
\end{aligned}
$$

respectivamente. La formulación de elemento finito del problema (5.19) es: encontrar $\lambda_{h} \in W_{0 h}$ tal que

$$
\int_{\mathcal{D}}\left(\mathbb{S}^{-1} \nabla \lambda_{h}\right) \cdot \nabla \phi_{h} d x=-\int_{\mathcal{D}} \mathbf{v}_{h}^{0} \cdot \nabla \phi_{h} \forall \phi_{h} \in W_{0 h},
$$

donde $\mathbf{v}_{h}^{0}$ es la función de interpolación en $W_{h} \times W_{h}$ que aproxima $\mathbf{v}^{0}$. Se sabe que la aproximación lineal a trozos $\lambda_{h}$ es una aproximación de segundo orden [7]. Sea $N$ el número total de vértices $x_{k}$ en la triangulación $\tau_{h}$ de $\bar{D}$, y supongamos una numeración de los vértices mencionados anteriormente. Entonces, una base del espacio $W_{k}$ es la colección $\beta_{h}=\left\{\phi_{k}\right\}_{1 \leq k \leq N}$ de funciones gorro $\phi_{k}$ [7] asociadas a aquellos vértices donde $\phi_{k}$ es una función lineal a trozos tal que $\phi_{k}\left(x_{l}\right)=\delta_{k l}$ para $l=1, \ldots N$. El soporte de estas funciones es la unión de triángulos en $\tau_{h}$ que contienen $x_{k}$ como vértice. De la misma manera, una base del espacio $W_{0 h}$ es

$$
\beta_{0 h}=\left\{\phi_{k} \in \beta_{h}: \phi_{k}\left(x_{l}\right)=0 \text { si } x_{l} \text { es un vértice en } \Gamma_{D}\right\}=\left\{\phi_{k} \in \beta_{h}: k=1,2, \ldots, N_{0}\right\}
$$

donde $N_{0}$ es el número de vértices que no pertenecen a $\Gamma_{D}$. Usando estas funciones base $\lambda_{k}(x)$ tiene la forma (5.26) donde $\left\{x_{k}\right\}_{k=1}^{N_{0}}$ es la colección de vértices en la triangulación que no pertenece a $\Gamma_{D}$. Si a $\lambda\left(x_{k}\right)$ la expresamos como $\lambda_{k}$ el problema (5.28) es equivalente a resolver el problema algebraico

$$
\sum_{l=1}^{N_{0}} a_{k l} \lambda_{l}=f_{k}, k=1, \ldots, N_{0}
$$

donde $a_{k l}=\left\langle\mathbb{S}^{-1} \nabla \phi_{k} \mid \nabla \phi_{l}\right\rangle_{\mathcal{D}}, f_{k}=-\left\langle\mathbf{v}_{h}^{0} \mid \nabla \phi_{k}\right\rangle_{\mathcal{D}}$. La matriz $a_{k l}$ mantiene las propiedades de $\mathbb{S}^{-1}$, i.e., $a_{k l}$ es simétrica y positiva definida. El problema algebraico (5.29) es resuelto por una versión del método de gradiente conjugado para sistemas lineales ralos [16]. En principio $\lambda_{h}$ proporciona la aproximación

$$
v_{h}^{i}=v_{h}^{0, i}+\frac{1}{\alpha_{i}^{2}} \frac{\partial \lambda_{h}}{\partial x^{i}}
$$

de $\mathbf{v}$ pero $\nabla \lambda_{h}$ no está definida en la frontera de la triangulación $\tau_{h}$ ya que $\lambda_{h}$ es una función lineal a trozas, así que $V_{h}^{i}$ puede ser calculada en un sentido débil de la siguiente manera [20]. Supongamos que $\partial \lambda_{h} / \partial x^{i}$ tiene la forma

$$
\frac{\partial \lambda_{h}}{\partial x^{i}}=\sum_{k=1}^{N_{0}} \tilde{\lambda}_{i k} \phi_{k}(x)
$$

de (5.29) tenemos

$$
\frac{\partial \lambda_{h}}{\partial x^{i}}=\sum_{k=1}^{N_{0}} \lambda_{k} \frac{\partial \phi_{k}(x)}{\partial x^{i}}
$$

Multiplicando por $\phi_{l}$ e integrando obtenemos el problema algebraico para las $\tilde{\lambda}_{i k}$

$$
\sum_{k=1}^{N_{0}} \tilde{\lambda}_{i k}\left\langle\phi_{k}, \phi_{l}\right\rangle=\sum_{k=1}^{N_{0}} \lambda_{k}\left\langle\frac{\partial \phi_{k}}{\partial x^{i}}, \phi_{l}\right\rangle \text { para } l=1, \ldots, N_{0} .
$$

Una forma más simple es calcular $u_{i} \equiv V_{h}^{i}-V_{h}^{0, i}$. Para $i=1,3$ encontrar $u_{i} \in W_{h}$ tal que

$$
\int_{\mathcal{D}} u_{i} \phi_{k} d x=\int_{\mathcal{D}} \frac{1}{\alpha_{i}^{2}} \frac{\partial \lambda}{\partial x^{i}} \phi_{k} d x \text { para } k=1,2, \ldots, N .
$$

La integral en el lado derecho puede calcularse exactamente por la regla del trapecio ya que $\partial \lambda / \partial x^{i}$ es constante en cada triángulo $T \in \tau_{h}$. Si el lado izquierdo es calculado con la misma regla obtenemos un sistema de ecuaciones diagonal cuya solución es inmediata. 
Nota 5.4 Un procedimiento común utilizado en modelos atmosféricos para simplificar las condiciones de frontera impuestas por la topografía, es el uso de coordenadas que siguen al terreno ó coordenadas $\sigma$ las cuales son constantes sobre la topografía [33], [35].

En este trabajo se usa

$$
\sigma=\frac{z-h(x)}{z_{\text {máx }}-h(x)}
$$

para trasformar el dominio $\mathcal{D}=\left[0, x_{\text {máx }}\right] \times\left[h(x), z_{\text {máx }}\right]$ en el espacio $x z$, en el dominio $\mathcal{D}_{\sigma}=\left[0, x_{\text {máx }}\right] \times$ $\left[0, \sigma_{\text {máx }}=1\right]$ en el espacio $x \sigma$. Entonces se define una malla uniforme $\left\{x^{(k)}, \sigma^{(k)}\right\}_{k=1}^{N}$, cuya imagen bajo la trasformación inversa $z=h(x)+\left[z_{\text {máx }}-h(x)\right] \sigma$ proporciona la malla de los elementos finitos $\left\{x^{(k)}, z^{(k)}\right\}_{k=1}^{N}$ en el espacio $x z$. Todos los cálculos fueron hechos en una malla de $81 \times 81$ puntos; el método de elemento finito se implementó en Fortran 77 utilizando una computadora de 32 bytes.

\subsection{Ejemplos numéricos}

Consideramos el dominio $\mathcal{D}=[0,10] \times[h(x), 10] \mathrm{km}^{2}$. El campo $\mathbf{v}_{\text {true }}=u_{\text {true }} \mathbf{i}+w_{\text {true }} \mathbf{k}$ es obtenido con el método de mapeo conforme donde $V_{0}$ viene de $(4.25)$ con $u_{\text {true }}=10 \mathrm{~ms}^{-1}$ a $(x=0, z=10 \mathrm{~km})$. Para simplificar la discusión proponemos el campo inicial $\mathbf{v}^{0}=u_{\text {true }}(x, y) \mathbf{i}$ donde hacemos $w^{0}=0$ ya que en situaciones reales la componente vertical $w_{\text {true }}$ no es medida. Este ejemplo nos permite tener el mejor campo ajustado que el MMC puede dar. El punto importante es, entonces, si el esquema puede generar el campo verdadero, en particular, la componente vertical $w_{\text {true }}$. Siguiendo [35] el campo ajustado $\mathbf{v}\left(\alpha^{2}\right)$ es reportado

\begin{tabular}{|c|c|c|}
\hline$\left[x_{\text {mín }}^{i}, x_{\text {máx }}^{i}\right]$ & {$\left[\sigma_{\text {mín }}^{i}, \sigma_{\text {máx }}^{i}\right.$} & {$\left[I\left(\Gamma_{i}\right)_{\text {mín }}, I\left(\Gamma_{i}\right)_{\text {máx }}\right]$} \\
\hline $\begin{array}{ll}0,02 & 0,99]\end{array}$ & {$\left[\begin{array}{ll}0,4 & 0,6\end{array}\right]$} & {$\left[6 \times 10^{-4} \quad 5 \times 10^{-3}\right]$} \\
\hline 0,6$]^{\circ}$ & $0,6]$ & {$\left[\begin{array}{ll}2 \times 10^{-4} & 10^{-3}\end{array}\right]$} \\
\hline
\end{tabular}

Cuadro 5.1: Flujo $I\left(\Gamma_{i}\right)$ a través de la región $\mathcal{D}_{i}=\left[x_{\text {mín }}^{i}, x_{\text {máx }}^{i}\right] \times\left[\sigma_{\text {mín }}^{i}, \sigma_{\text {máx }}^{i}\right]$ con $\alpha^{2} \in\left[10^{-4}, 10^{+6}\right]$.

en términos del parámetro $\alpha=\alpha_{1} / \alpha_{3}$, donde proponemos $\alpha_{1}=1$ así como $\alpha^{2}=\alpha_{3}^{-2}$. La figura (5.2) muestra los campos $\mathbf{v}_{\text {true }} \mathrm{y} \mathbf{v}\left(\alpha^{2}=1\right)$ sobre la topografía $h(x)=h_{0}+h_{1} \cos \omega x$ estimadas con $\operatorname{IP}^{3} \mathrm{~S} s(x)$ con $p=0,8$. Detalles de la figura (5.2) se muestran en la figura (5.3). donde se puede ver un diferencia significativa entre $\mathbf{v}_{\text {true }} \mathrm{y} \mathbf{v}\left(\alpha^{2}=1\right)$.

Sherman [23] seguida por otros autores [8,23], sugiere que $\alpha$ puede ser proporcional a la magnitud del cociente $w_{\text {true }} / u_{\text {true }}$. Aplicando este criterio calculamos el cociente $\alpha_{\text {true }}^{(k)} \equiv\left|w_{\text {true }}^{(k)} / u_{\text {true }}^{(k)}\right|$ en la malla $\left\{x^{(k)}, z^{(k)}\right\}_{k=1}^{N=6561}$ usada para estimar $\mathbf{v}\left(\alpha^{2}\right)$ con EF. El resultado es $\operatorname{mín}\left(\alpha_{\text {true }}^{(k)}\right)=0,7, \operatorname{máx}\left(\alpha_{\text {true }}^{(k)}\right)=17,5$, $\left\langle\alpha_{\text {true }}\right\rangle=\frac{1}{N} \sum_{k=1}^{N} \alpha_{\text {true }}^{(k)}=0,8$ con desviación estándar 1,8 , de tal forma que

$$
10^{-1} \leq\left\langle\alpha_{\text {true }}\right\rangle^{2} \leq 10^{+2}
$$

El valor $\left\langle\alpha_{\text {true }}\right\rangle^{2} \sim 10^{0}$ proporciona el campo $\mathbf{v}\left(\alpha^{2}=1\right)$ de las figura (5.2) y (5.3). Consideremos los valores extremos. El campo $\mathbf{v}\left(\alpha^{2}=10^{-1}\right)$ es casi igual a $\mathbf{v}\left(\alpha^{2}=1\right)$. la figura (5.4) muestra que el campo $\mathbf{v}\left(10^{-2}\right)$ es peor que el campo $\mathbf{v}\left(\alpha^{2}=1\right)$ mientras la figura (5.5) muestra que $\mathbf{v}\left(\alpha^{2}=10^{+2}\right)$ es más cercano a $\mathbf{v}_{\text {true }}$. Siguiendo esta tendencia calculamos $\mathbf{v}\left(\alpha^{2}=10^{+6}\right)$ el cual es dibujado en la figura (5.5). Ahí vemos que $\mathbf{v}\left(\alpha^{2}=10^{+6}\right)$ es casi igual a $\mathbf{v}_{\text {true }}$. Todos los cálculos fueron hechos con la misma malla, así que las diferencias entre los campos ajustados se deben a los valores de $\alpha^{2}$.

Como un segundo ejemplo, la figura (5.5) muestra los campos $\mathbf{v}_{\text {true }} \mathrm{y} \mathbf{v}\left(\alpha^{2}=10^{+6}\right)$ sobre la topografía $h(x)$ definida por datos de elevación del terreno de la base de datos GTOPO [41]. Los detalles de la misma figura son mostrados en figura (5.5). Observamos que el campo ajustado $\mathbf{v}\left(\alpha^{2}=10^{+6}\right)$ es de hecho una correcta aproximación de $\mathbf{v}_{\text {true }}$. Una medida conveniente de la cercanía entre $\mathbf{v}_{\text {true }} \mathrm{y} \mathbf{v}\left(\alpha^{2}\right)$ es dada por la distancia en $L^{2}(\mathcal{D})$ entre sus componentes

$$
d\left(u, \alpha^{2}\right)=\left[\int_{\mathcal{D}}\left|u_{\text {true }}-u\left(\alpha^{2}\right)\right|^{2} d x\right]^{1 / 2}
$$


La figura (5.9) muestra que $d\left(u, \alpha^{2}\right)$ y $d\left(w, \alpha^{2}\right)$ tienden monótona y asintóticamente al valor mínimo, cercano a 0,01 , conforme $\alpha^{2} \rightarrow \infty$, a su vez, valores pequeños de $\alpha^{2}$ proporciona malos campos ajustados. Esto confirma la bondad de $\mathbf{v}\left(\alpha^{2}=10^{+6}\right)$. La misma figura muestra un mejor ajuste para la componente horizontal que para la componente vertical, como se esperaba, debido a que al principio eliminamos la componente vertical. La figura (5.10) muestra las gráficas de $\alpha^{2}$ vs. $\left\|\mathbf{v}-\mathbf{v}\left(\alpha^{2}\right)\right\|_{\mathcal{D}}^{2} \mathrm{y}\left\|\mathbf{v}-\mathbf{v}\left(\alpha^{2}\right)\right\|_{\mathcal{D} S}^{2}$, ambas normas tienden a $10^{-2}$ mientras $\alpha^{2} \rightarrow 10^{+6}$ pero la norma $\left\|\mathbf{v}-\mathbf{v}\left(\alpha^{2}\right)\right\|_{\mathcal{D S}}^{2}$ decrece más rápidamente ya que su minimización es el criterio usado para calcular $\mathbf{v}\left(\alpha^{2}\right)$. Veamos qué tan bien satisface el campo $\mathbf{v}\left(\alpha^{2}\right)$ la ecuación de conservación de la masa. De acuerdo a la identidad

$$
\int_{\mathcal{D}_{i}} \nabla \cdot \mathbf{v} d x=\int_{\Gamma_{i}} \mathbf{v} \cdot \mathbf{n} d s
$$

es suficiente calcular el flujo

$$
I\left(\Gamma_{i}\right)=\int_{\Gamma_{i}} \mathbf{v}\left(\alpha^{2}\right) \cdot \mathbf{n} d s
$$

sobre algunas subregiones $\mathcal{D}_{i}$ de $\mathcal{D}$ sobre el espacio $x \sigma$ (NOTA 2). Los resultado reportados en el cuadro (5.1) muestran que $I\left(\Gamma_{i}\right)$ va de $2 \times 10^{-4}$ a $5 \times 10^{-3}$ para $\alpha^{2}=\left(1 / \alpha_{3}\right)^{2} \in\left[10^{-4}, 10^{6}\right]$ así que $\mathbf{v}\left(\alpha^{2}\right)$ casi satisface la ecuación (5.1). 


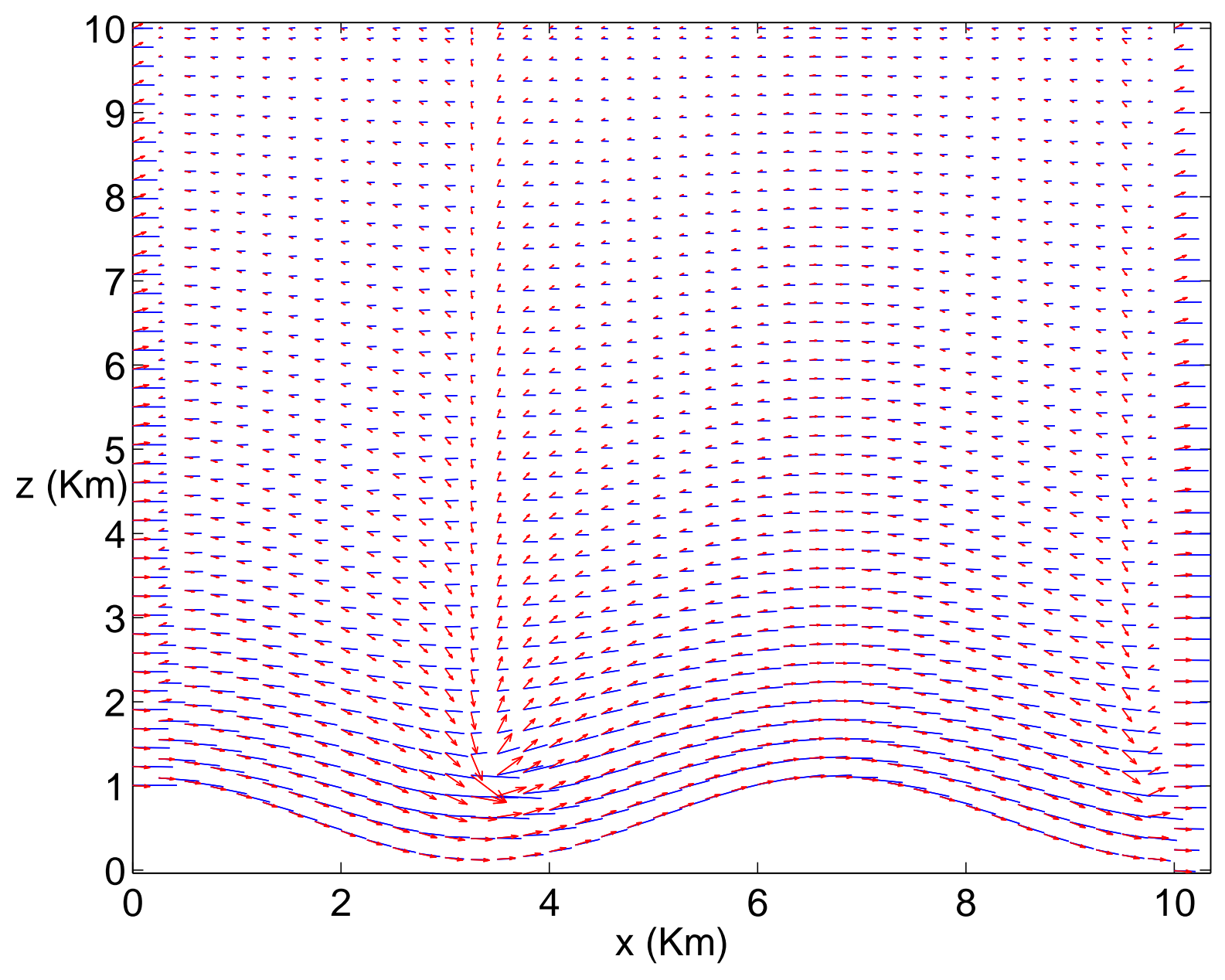

Figura 5.2: Campos $\mathbf{v}_{\text {true }}(\rightarrow)$ y $\mathbf{v}\left(\alpha^{2}=1\right)(-)$. 


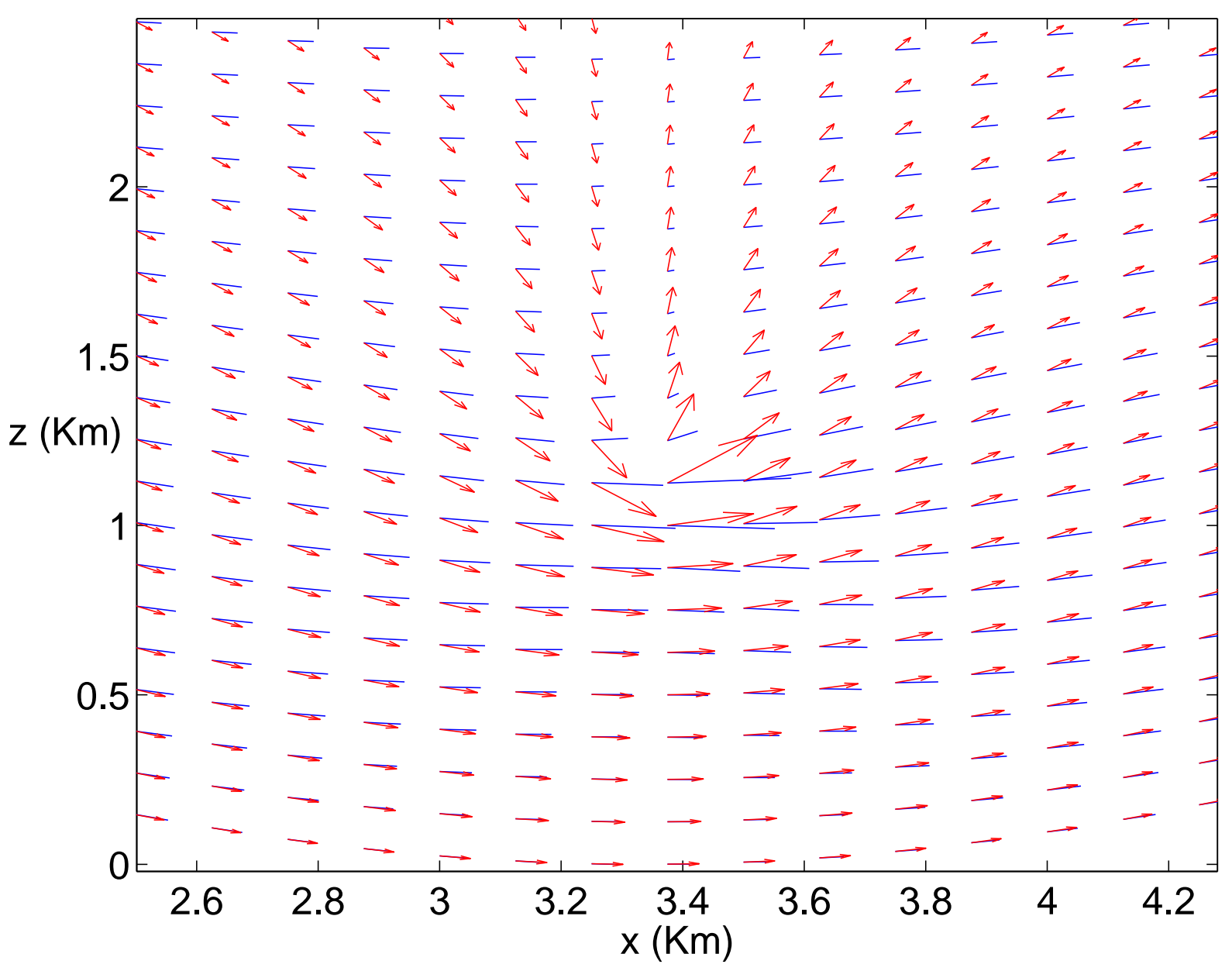

Figura 5.3: Detalle de la figura (5.2). 


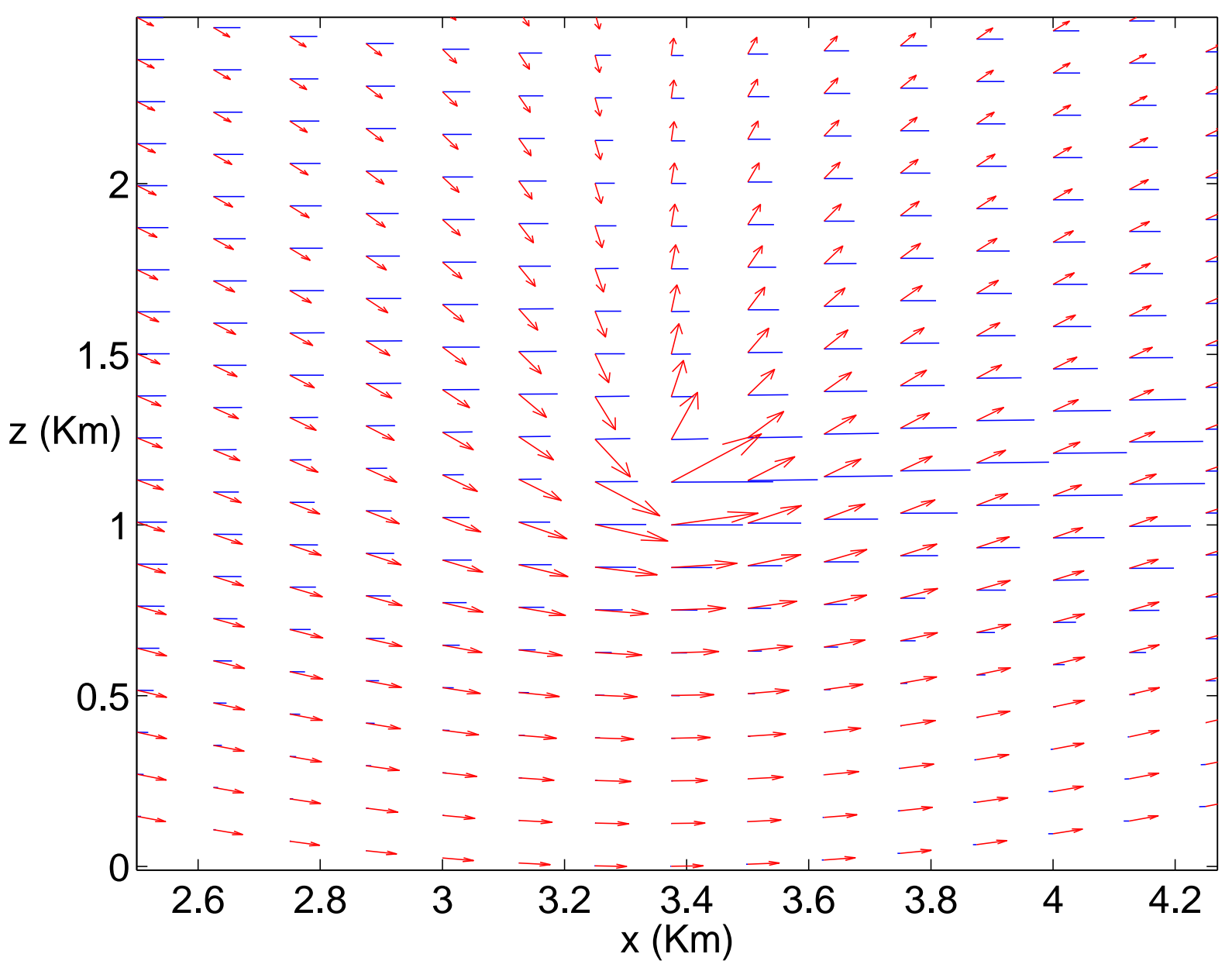

Figura 5.4: Campos $\mathbf{v}_{\text {true }}(\rightarrow)$ y v $\left(\alpha^{2}=10^{-2}\right)(-)$. 


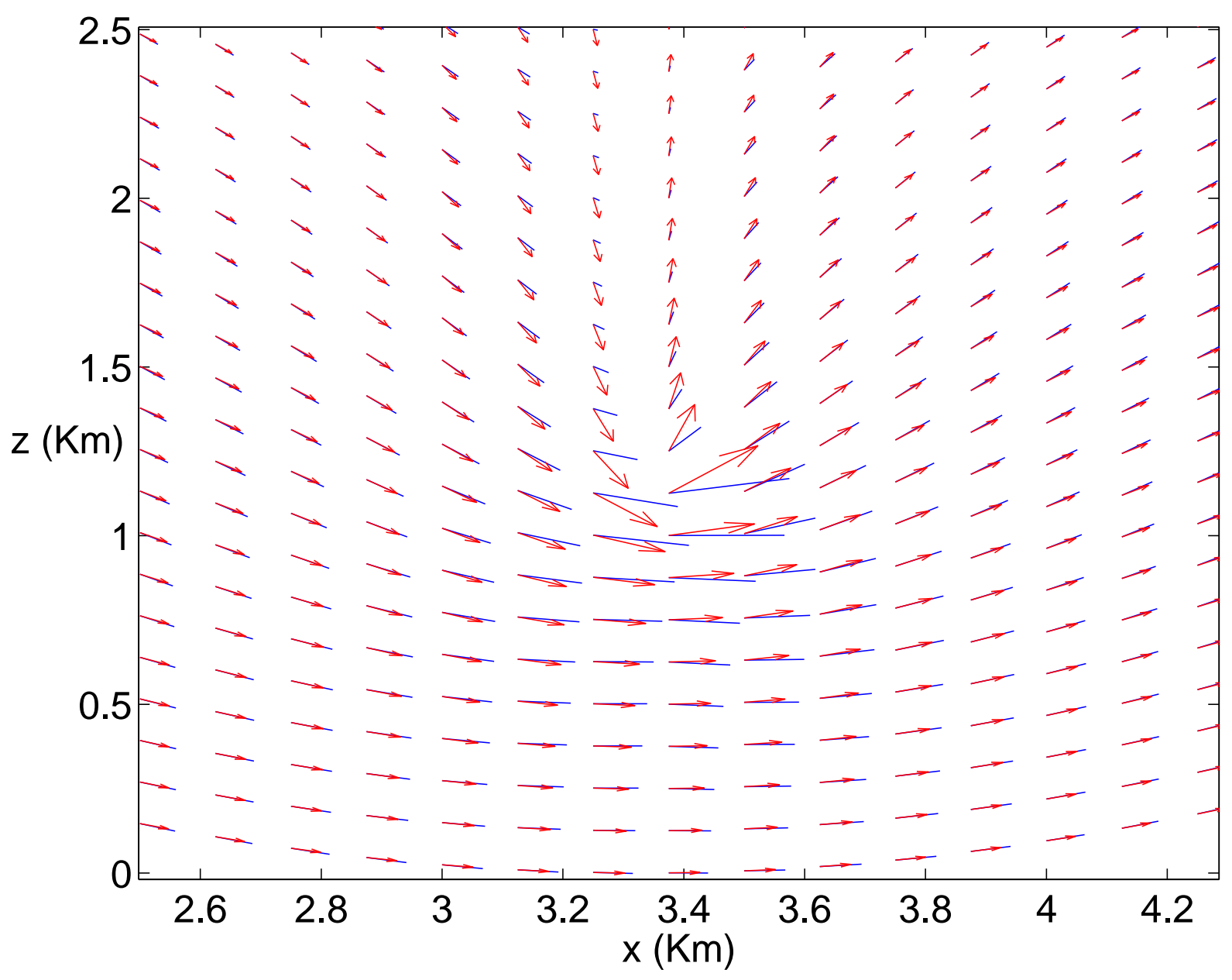

Figura 5.5: Campos $\mathbf{v}_{\text {true }}(\rightarrow)$ y $\mathbf{v}\left(\alpha^{2}=10^{+2}\right)(-)$. 


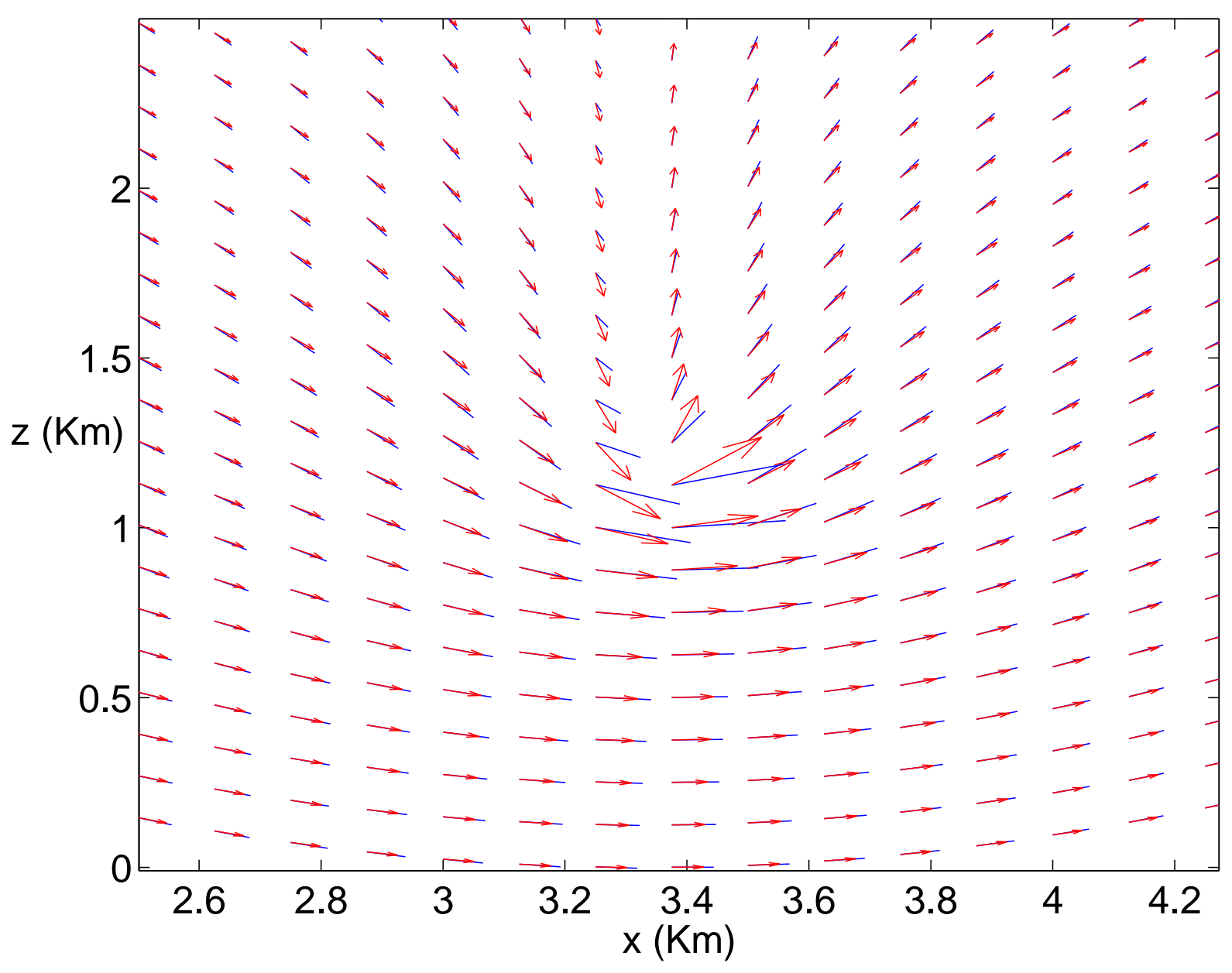

Figura 5.6: Campos $\mathbf{v}_{\text {true }}(\rightarrow)$ y v $\left(\alpha^{2}=10^{-6}\right)(-)$. 


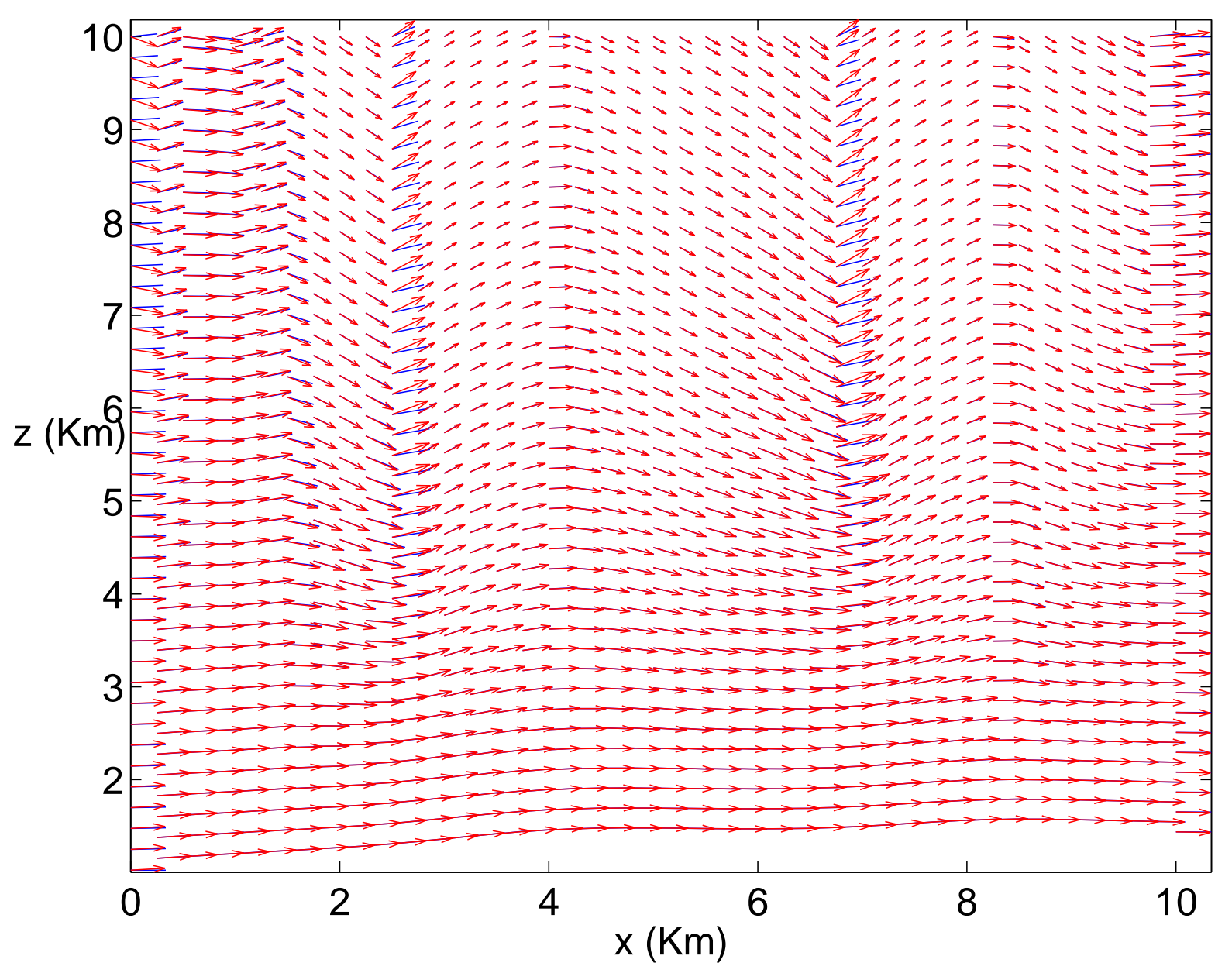

Figura 5.7: Campos $\mathbf{v}_{\text {true }}(\rightarrow)$ y v $\left(\alpha^{2}=10^{+6}\right)(-)$. 


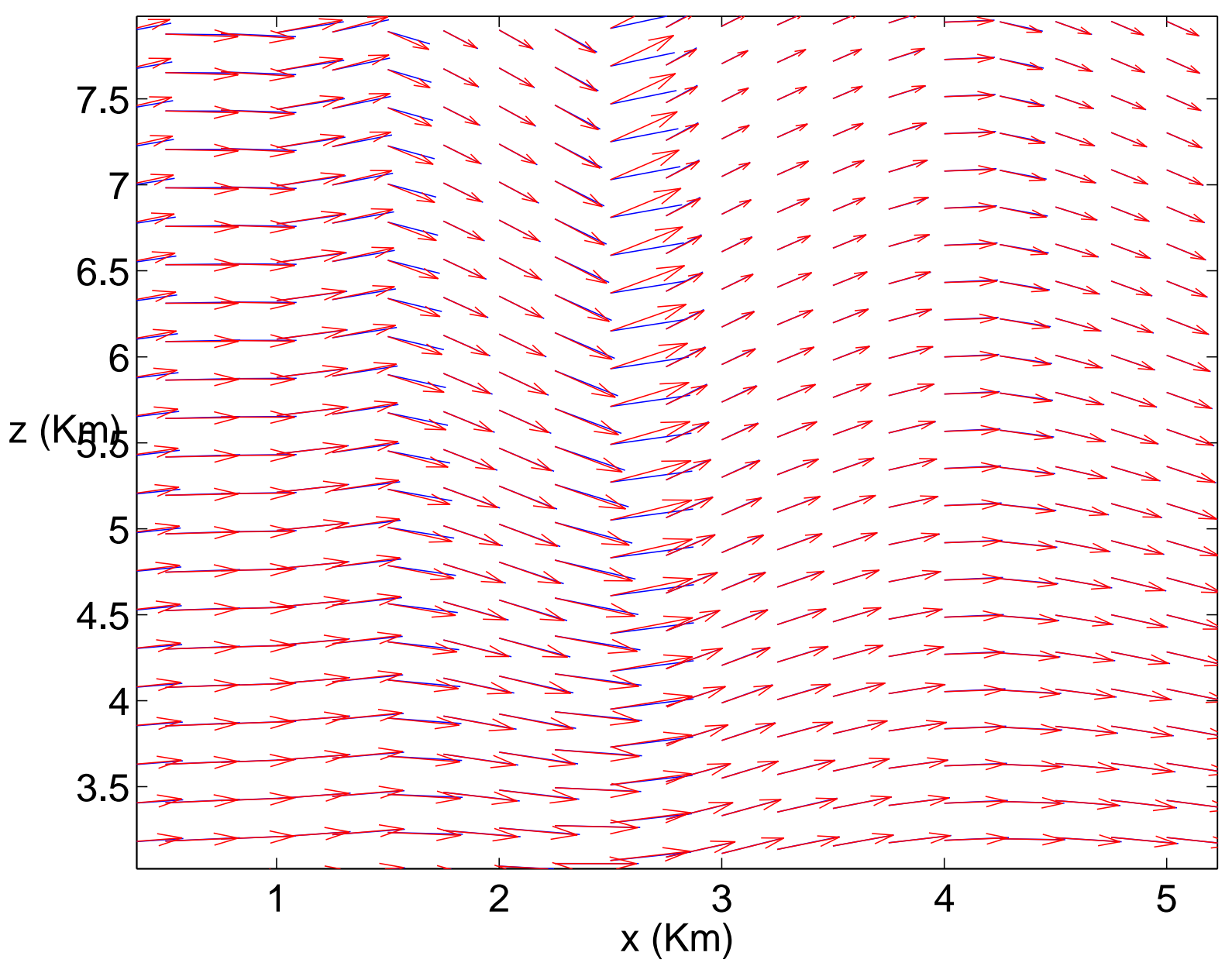

Figura 5.8: Detalles de Fig. 5.5. 


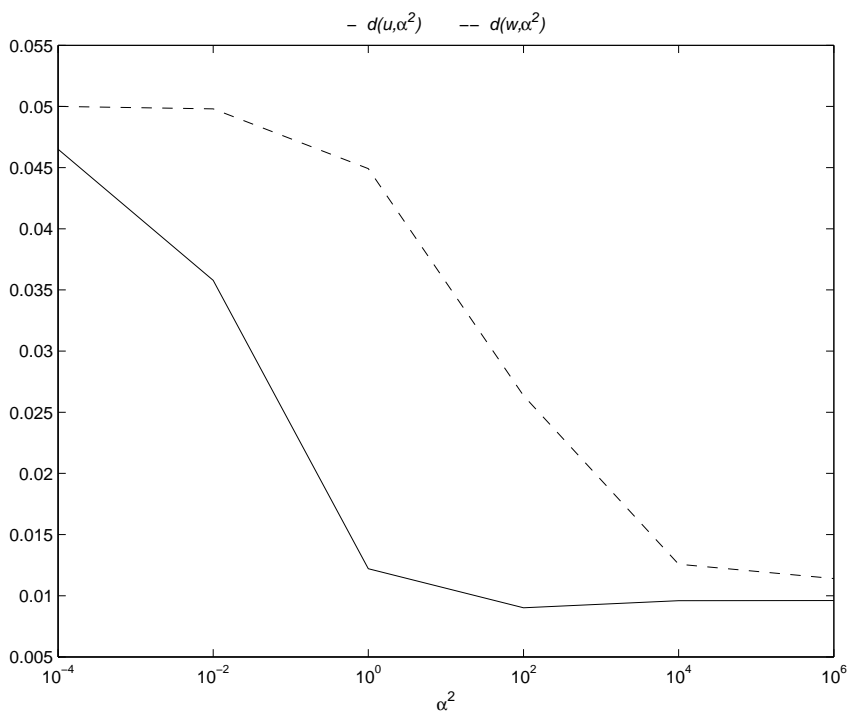

Figura 5.9: Distancias $d\left(u, \alpha^{2}\right)(-)$ y $d\left(w, \alpha^{2}\right)(--)$ entre componentes $\mathbf{v}_{\text {true }} \mathrm{y} \mathbf{v}\left(\alpha^{2}\right)(-)$.

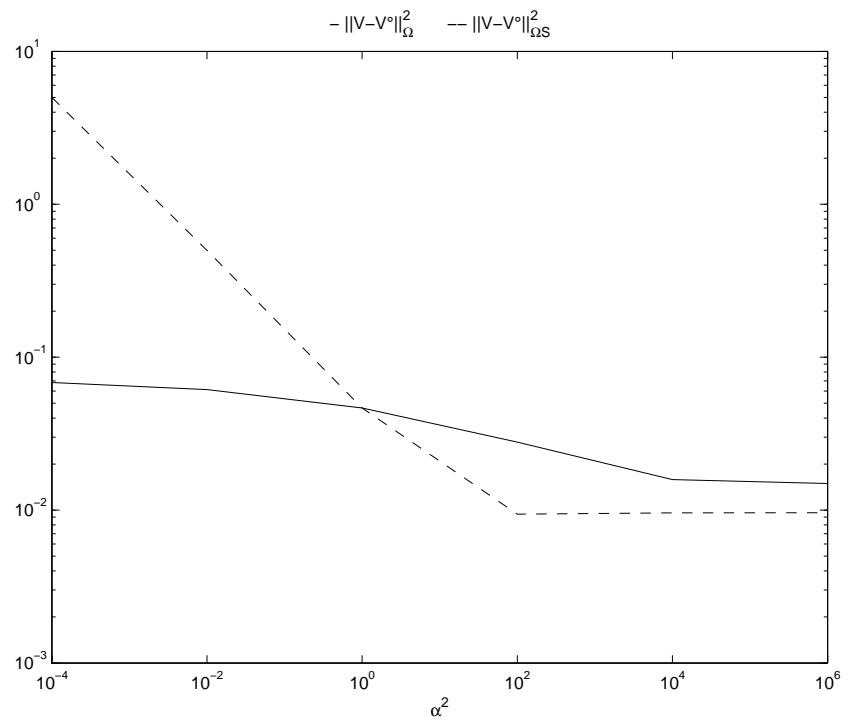

Figura 5.10: Distancias $\left\|\mathbf{v}_{\text {true }}-\mathbf{v}\left(\alpha^{2}\right)\right\|_{D}^{2}$ y $\left\|\mathbf{v}_{\text {true }}-\mathbf{v}\left(\alpha^{2}\right)\right\|_{D S}^{2}$. 


\section{Capítulo 6}

\section{Formulación tensorial}

En meteorología una forma estándar de simplificar las condiciones de frontera impuestas por el terreno es introducir un nuevo sistema de coordenadas generalizadas $y^{k}$ las cuales el tienen el nombre de coordenadas $\sigma$ ó coordenadas que siguen al terreno. Veamos algunos ejemplos

1. En [22] Moussiopoulus y Flassak (1983) usan

$$
\begin{aligned}
& y^{1}=x \quad y^{2}=y \quad y^{3}=\sigma=\frac{H-z^{3}}{H-h(x, y)} \quad \text { con } \quad h(x, y) \leq z \leq H(x, y) \\
& x=y^{1} \quad y=y^{2} \quad z=H+\left[h\left(y^{1}, y^{2}\right)-H\right] y^{3}
\end{aligned}
$$

2. En [15] Kitada et. al. (1983) usan

$$
\begin{array}{rlrl}
y^{1}=x & y^{2}=y & y^{3}=\frac{z-h(x, y)}{H(x, y)-h(x, y)} \quad \text { con } \quad h(x, y) \leq z \leq H(x, y) \\
x=y^{1} & y=y^{2} & z & =y^{3}\left[H\left(y^{1}, y^{2}\right)-h\left(y^{1}, y^{2}\right)\right]+h\left(y^{1}, y^{2}\right) .
\end{array}
$$

3. En [40] Kitada et.al. (1986) usan

$$
\begin{array}{llc}
y^{1}=x & y^{2}=y & y^{3}=\frac{z-h(x, y)}{\Delta H} \quad \text { con } \quad h(x, y) \leq z \leq \Delta H+h(x, y) \\
x=y^{1} & y=y^{2} & z=y^{3} \Delta H+h\left(y^{1}, y^{2}\right)
\end{array}
$$

donde $\Delta H$ es la altura de la frontera superior $\mathcal{D}$ y a la cual se le asigna un valor de $10^{3} \mathrm{~m}$.

4. Bernard et. al. (1987) consideran [2]

$$
\begin{array}{rlrl}
y^{1}=x & y^{2}=y & y^{3}=\frac{z_{t}-z}{z_{t}-z_{s}(x, y)} \quad \text { con } \quad z_{s}(x, y) \leq z \leq z_{t} \\
x=y^{1} & y=y^{2} & z & =z_{t}-y^{3}\left[z_{t}-z_{s}\left(y^{1}, y^{2}\right)\right]
\end{array}
$$

donde $z_{t}$ es la altura (constante) de la frontera superior y $z_{s}(x, y)$ es la altura del terreno.

5. El modelo ARPS [44] usa las coordenadas

$$
\begin{array}{lll}
y^{1}=x & y^{2}=y & y^{3}=H \frac{z-h(x, y)}{H-h(x, y)}+z_{0} \quad \text { con } \quad h(x, y) \leq z \leq H \\
x=y^{1} & y=y^{2} & z=\frac{H-h\left(y^{1}, y^{2}\right)}{H}\left(y^{3}-z_{0}\right)+h\left(y^{1}, y^{2}\right)
\end{array}
$$


En cada uno de lo ejemplos anteriores las coordenadas $\sigma$ tienen la forma

$$
y^{1}=x \quad y^{2}=y \quad y^{3}=y^{3}(x, y, z)
$$

o, equivalentemente,

$$
x=y^{1} \quad y=y^{2} \quad z=z\left(y^{1}, y^{3}, y^{2}\right)
$$

donde la coordenada $\sigma$ toma un valor constante tanto en la superficie terrestre,

$$
\sigma[x, y, z=h(x, y)]=\text { cte } \quad \text { sobre } \Gamma_{N}
$$

como en la frontera superior $z=z_{\max }$,

$$
\sigma\left[x, y, z=z_{\max }\right]=c t e \quad \text { sobre } \Gamma_{D} .
$$

Las coordenadas $x, y$ están acotadas por valores constantes. Así, en el espacio físico el dominio $\mathcal{D}$ tiene la forma

$$
\mathcal{D}=\left\{(x, y, z): x_{\min } \leq x \leq x_{\max } \quad y_{\min } \leq y \leq y_{\max } \quad h(x, y) \leq z \leq z_{\max }\right\}
$$

y su imagen bajo la transformación $y^{k}=y^{k}(x)$ es un paralelepípedo

$$
\mathcal{D}_{y}=\left\{\left(y^{1}, y^{2}, y^{3}\right): x_{\min } \leq y^{1} \leq x_{\max } \quad y_{\min } \leq y^{2} \leq y_{\max } \quad \sigma_{\min } \leq y^{3} \leq \sigma_{\max }\right\}
$$

\subsection{Base covariante $\tau_{j}$, contravariante $\eta^{j}$ y tensor métrico $\mathbb{G}$}

En esta seccion reproducimos el procedimiento usado en [25] para obtener la formulación tensorial de el problema variacional. Usaremos la convención de suma sobre índices repetidos. Comencemos con los conceptos básicos para construir la estructura invariante de la ecuación de continuidad profunda. Sean $x^{i}$ las coordenadas en el sistema $X$, de un punto $P=\left(x^{1}, x^{2}, x^{3}\right)$. Supongamos que las coordenadas $y^{i}$ son las coordenadas, en el sistema $Y$, del mismo punto $p=\left(y^{1}, y^{2}, y^{3}\right)$. Ya que estamos describiendo la posición del mismo punto con dos conjuntos diferentes de funciones es lógico pensar que existe una relación entre ambos

$$
x^{i}=x^{i}\left(y^{j}\right) .
$$

El vector de posición de un punto con coordenadas $\left(x^{1}, x^{2}, x^{3}\right)$ está dado por

$$
\mathbf{r}=x^{i} \hat{\mathbf{x}}^{i}(t)
$$

en donde los vectores base y las coordenadas dependen implícitamente del tiempo ya que el sistema $X$ es un sistema fijo a la tierra y por lo tanto no es inercial. Usando (6.2) vemos que el vector de posición es función de las coordenadas $y^{i}$

$$
\mathbf{r}\left(y^{j}\right)=x^{i}\left(y^{j}\right) \hat{\mathbf{x}}^{i}(t) .
$$

La variación de la j-ésima coordenada $y^{j}$ define una curva en $\mathbb{R}^{3}$ que llamaremos curva coordenada. Los vectores tangentes a las curvas coordenadas que pasan el punto $\left(y^{1}, y^{2}, y^{3}\right)$ están dados por

$$
\tau_{j}=\frac{\partial \mathbf{r}}{\partial y^{j}}=\frac{\partial x^{i}}{\partial y^{j}} \hat{\mathbf{x}}^{i}(t)
$$

y reciben el nombre de vectores base covariantes. De acuerdo con la definición de matriz jacobiana $\mathbb{J}$

$$
\mathbb{J}_{i j}=\frac{\partial x^{i}}{\partial y^{j}}
$$

podemos escribir a los vectores base covariantes como combinación lineal de los vectores base $\hat{\mathbf{x}}^{i}$

$$
\tau_{j}=\mathbb{J}_{j i}^{T} \hat{\mathbf{x}}^{i}(t) .
$$


Si el jacobiano $J=\operatorname{det}(\mathbb{J})$ de la transformación (6.2) no es nulo en el punto $\left(y^{1}, y^{2}, y^{3}\right)$, el conjunto $\left\{\tau_{j}\right\}_{j=1,2,3}$ es linealmente independiente, lo que valida el uso del término "base" al referirnos a dicho conjunto. En este caso el teorema de la función implícita afirma que las coordenadas $y^{j}$ pueden despejarse de (6.2) lo que nos da las ecuaciones de transformación inversa

$$
y^{j}=y^{j}\left(x^{i}\right)
$$

El gradiente de cada función $y^{j}$ da un vector normal a la superficie definida por $y^{j}=c t e$, dichos vectores forman la base contravariante

$$
\eta^{j}=\nabla y^{j}=\frac{\partial y^{j}}{\partial x^{i}} \hat{\mathbf{x}}^{i}
$$

o en forma matricial

$$
\eta^{j}=\mathbb{J}_{j i}^{-1} \hat{\mathbf{x}}^{i}
$$

Dado que $\left\{\eta^{j}\right\}_{j=1,2,3}$ es una base, podemos escribir la base $\left\{\hat{\mathbf{x}}^{i}\right\}_{i=1,2,3}$ en términos de ésta

$$
\hat{\mathbf{x}}^{i}=\mathbb{J}_{i j} \eta^{j}
$$

sustituyendo en (6.3) obtenemos la relación entre la base covariante y la contravariante

$$
\tau_{i}=\frac{\partial x^{k}}{\partial y^{i}} \frac{\partial x^{k}}{\partial y^{j}} \eta^{j}
$$

donde aparecen los elementos

$$
g_{i j}=\frac{\partial x^{k}}{\partial y^{i}} \frac{\partial x^{k}}{\partial y^{j}}
$$

del tensor métrico $\mathbb{G}=\left\{g_{i j}\right\}$, con lo que obtenemos

$$
\tau_{i}=g_{i j} \eta^{j}
$$

En forma matricial podemos escribir

$$
\mathbb{G}=\mathbb{J}^{T} \mathbb{J}
$$

\subsection{Forma covariante y contravariante de un vector}

La expresión de un vector $\mathbf{A}$ en la base $\tau_{j}$

$$
\mathbf{A}=\sum_{j} A^{j}(y) \tau_{j}
$$

recibe el nombre de forma contravariante de $\mathbf{A}$ y nos referimos a los coeficientes $A^{j}(y)$ como las componentes contravariantes de $\mathbf{A}$ en el sistema de coordenadas $y$. La expresión de $\mathbf{A}$ en la base $\eta^{j}$

$$
\mathbf{A}=\sum_{j} A_{j}(y) \eta^{j}
$$

se llama forma covariante de $\mathbf{A}$ y los coeficientes $A_{j}(y)$ reciben el nombre de componentes covariantes de $\mathbf{A}$ en el sistema de coordenadas $y$. De acuerdo con lo anterior, las formas covariante y contravariante de A en el sistema de coordenadas $x^{1}, x^{2}, x^{3}$ son, respectivamente,

$$
\mathbf{A}=\sum_{i} A^{i}(x) \hat{\mathbf{x}}_{i}
$$

y

$$
\mathbf{A}=\sum_{i} A_{i}(x) \hat{\mathbf{x}}^{i}
$$


pero dado que la base $\hat{\mathbf{x}}_{i}$ es recíproca de si misma $\left(\hat{\mathbf{x}}_{i}=\hat{\mathbf{x}}^{i}\right)$ tenemos

$$
A^{i}(x)=A_{i}(x) .
$$

Para obtener la relación entre componentes cartesianas, covariantes y contravariantes de $\mathbf{A}$ consideremos su expresión en términos de las tres bases

$$
\begin{aligned}
\mathbf{A} & =A_{i}(x) \hat{\mathbf{x}}^{i} & & \text { base canónica } \\
& =A^{j}(y) \tau_{j} & & \text { forma contravariante } \\
& =A_{k}(y) \eta^{k} & & \text { forma covariante. }
\end{aligned}
$$

Igualando estas expresiones obtenemos

$$
\begin{aligned}
& A_{i}(x) \hat{\mathbf{x}}^{i}=A^{j}(y) \tau_{j} \\
& A_{i}(x) \hat{\mathbf{x}}^{i}=A_{k}(y) \eta^{k} \\
& A^{j}(y) \tau_{j}=A_{k}(y) \eta^{k} .
\end{aligned}
$$

Utilicemos las leyes de transformación entre bases para encontrar las leyes de transformación entre componentes, de (6.3) y de (6.8a) tenemos

$$
A^{i}(x) \frac{\partial y^{j}}{\partial x^{i}} \tau_{j}=A^{j}(y) \tau_{j}
$$

igualando componentes obtenemos la ley de transformación contravariante

$$
A^{j}(y)=A^{i}(x) \frac{\partial y^{j}}{\partial x^{i}} .
$$

De $(6.5)$ y de $(6.8 \mathrm{~b})$ tenemos

$$
A_{i}(x) \frac{\partial x^{i}}{\partial y^{k}} \eta^{k}=A_{k}(y) \eta^{k}
$$

igualando componentes obtenemos la ley de transformación covariante

$$
A_{k}(y)=A_{i}(x) \frac{\partial x^{i}}{\partial y^{k}} .
$$

De (6.8c) y de (6.6) tenemos

$$
A^{j}(y) g_{j k} \eta^{k}=A_{k}(y) \eta^{k}
$$

igualando componentes obtenemos la propiedad del tensor métrico de "bajar"índices

$$
A_{k}(y)=A^{j}(y) g_{j k} .
$$

Si multiplicamos $(6.11)$ por la inversa del tensor métrico $\left(\mathbb{G}^{-1}\right)_{i j}=g^{i j}$ obtenemos la propiedad del tensor métrico inverso de subir índices

$$
A^{j}(y)=A_{k}(y) g^{k j} .
$$

Una propiedad importante de las bases covariante y contravariante es la de reciprocidad. Calculemos el producto escalar entre un vector base covariante y otro vector base contravariante $\tau_{i} \cdot \eta^{j}$. Usando la regla de transformación entre bases tenemos

$$
\tau_{i} \cdot \eta^{j}=\frac{\partial x^{k}}{\partial y_{i}} \hat{\mathbf{x}}^{k} \cdot \frac{\partial y^{j}}{\partial x^{l}} \hat{\mathbf{x}}^{l}=\frac{\partial x^{k}}{\partial y_{i}} \frac{\partial y^{j}}{\partial x^{l}} \hat{\mathbf{x}}^{k} \cdot \hat{\mathbf{x}}^{l}
$$

como $\hat{\mathbf{x}}^{k}$ y $\hat{\mathbf{x}}^{l}$ son ortogonales entre sí, la expresión anterior se reduce a

$$
\tau_{i} \cdot \eta^{j}=\frac{\partial x^{k}}{\partial y_{i}} \frac{\partial y^{j}}{\partial x^{l}} \delta_{l}^{k}=\frac{\partial x^{k}}{\partial y_{i}} \frac{\partial y^{j}}{\partial x^{k}}=\frac{\partial y^{j}}{\partial y_{i}}=\delta_{i}^{j}
$$


donde usamos la regla de la cadena. La expresión

$$
\tau_{i} \cdot \eta^{j}=\delta_{i}^{j}
$$

es la propiedad de reciprocidad. Usemos esta propiedad para calcular el producto escalar de dos vectores cualquiera. Sean A y B dos vectores, uno en su forma contravariante y el otro en su forma covariante, entonces el producto escalar es

$$
\mathbf{A} \cdot \mathbf{B}=A^{i}(y) \tau_{i} \cdot B_{j}(y) \eta^{j}=A^{i}(y) B_{j}(y) \tau_{i} \cdot \eta^{j}=A^{i}(y) B_{j}(y) \delta_{i}^{j} ;
$$

es decir,

$$
\mathbf{A} \cdot \mathbf{B}=A^{i}(y) B_{i}(y)
$$

que es la regla para el producto escalar generalizado.

La velocidad de una partícula tiene una representación natural en la base $\tau_{j}$. Si el vector de posición con respecto al sistema $x^{1} x^{2} x^{3}$ está dado por

$$
\mathbf{r}=x^{i}(y) \hat{\mathbf{x}}_{i}
$$

La velocidad relativa a la Tierra está dada por $\mathbf{v}=\dot{x}^{i}(y) \hat{\mathbf{x}}_{i}$ (capítulo 1). Aplicando la regla de la cadena

$$
\dot{x}^{i}=\frac{d}{d t} x^{i}(y)=\dot{y}^{j} \frac{\partial x^{i}}{\partial y^{j}}
$$

y sustituyendo se obtiene

$$
\mathbf{v}=\hat{\mathbf{x}}_{i}\left(\dot{y}^{j} \frac{\partial x^{i}}{\partial y^{j}}\right)=\left(\hat{\mathbf{x}}_{i} \frac{\partial x^{i}}{\partial y^{j}}\right) \dot{y}^{j}=\tau_{j} \dot{y}^{j}
$$

por lo que los coeficientes $\dot{y}^{j}$ son las componentes contravariantes de v. En la literatura de mecánica de Lagrange los coeficientes $v^{j}=\dot{y}^{j}$ reciben el nombre de velocidades generalizadas. Así tenemos $v^{j}(y) \equiv \dot{y}^{j}$ tenemos

$$
\mathbf{v}=\sum_{j} \tau_{j} v^{j}
$$

\subsection{Forma tensorial de $\nabla, \nabla \cdot \mathbf{y} \nabla \cdot \rho_{0} \mathbf{v}=0$}

En el capítulo 1 mostramos que si en un sistema de referencia inercial $X$ definimos el operador gradiente como

$$
\nabla=\hat{\mathbf{X}}^{i} \frac{\partial}{\partial X^{i}}
$$

entonces conserva su estructura en cualquier sistema cartesiano $x^{i}$ fijo a la Tierra, esto es,

$$
\nabla=\hat{\mathbf{x}}^{i} \frac{\partial}{\partial x^{i}}
$$

Usando esta expresión junto con la regla de la cadena para calcular el gradiente de una función $f$ que depende explícitamente de las coordenadas $y^{i}$ se obtiene

$$
\nabla f=\hat{\mathbf{x}}^{i} \frac{\partial}{\partial x^{i}} f[y(x)]=\hat{\mathbf{x}}^{i} \frac{\partial y^{j}}{\partial x^{i}} \frac{\partial f(y)}{\partial y^{j}}
$$

donde aparece la definición de $\eta^{j}$ (6.5). Esto da la forma generalizada del vector gradiente

$$
\nabla f=\eta^{j} \frac{\partial f(y)}{\partial y^{j}}
$$

o bien

$$
\nabla=\eta^{j} \frac{\partial}{\partial y^{j}}
$$

lo que muestra que la forma natural del vector gradiente es su forma covariante. 
Para calcular la forma generalizada de la ecuación de continuidad profunda reescribámosla primero de la siguiente manera

$$
\nabla \cdot \mathbf{A}=0
$$

En el sistema $x$ ésta expresión se desarrolla de la siguiente manera

$$
\frac{\partial A^{i}(x)}{\partial x^{i}}=0
$$

nótese que A está escrito en su representación contravariante. Usando la regla de la cadena y la ley de transformación contravariante obtenemos el desarrollo en las coordenadas del sistema $y^{1}, y^{2}, y^{3}$

$$
\begin{aligned}
\frac{\partial y^{k}}{\partial x^{i}} \frac{\partial}{\partial y^{k}}\left[\frac{\partial x^{i}}{\partial y^{l}} A^{l}(y)\right] & =0 \\
\frac{\partial y^{k}}{\partial x^{i}}\left[\frac{\partial^{2} x^{i}}{\partial y^{k} \partial y^{l}} A^{l}(y)+\frac{\partial x^{i}}{\partial y^{l}} \frac{\partial A^{l}(y)}{\partial y^{k}}\right] & =0 \\
\frac{\partial^{2} x^{i}}{\partial y^{k} \partial y^{l}} \frac{\partial y^{k}}{\partial x^{i}} A^{l}(y)+\frac{\partial A^{l}(y)}{\partial y^{l}} & =0 .
\end{aligned}
$$

donde aparecen los símbolos de Christoffel los cuales se definen por

$$
\Gamma_{k l}^{n} \equiv \frac{\partial^{2} x^{i}}{\partial y^{k} \partial y^{l}} \frac{\partial y^{n}}{\partial x^{i}}
$$

Entonces, la forma generalizada de la ecuación de continuidad profunda es

$$
\Gamma_{k l}^{k} A^{l}(y)+\frac{\partial A^{l}(y)}{\partial y^{l}}=0
$$

Para obtener una forma más compacta de la última ecuación consideremos la siguiente relación entre $\Gamma_{k l}^{k}$ y el tensor métrico $\mathbb{G}$. El tensor métrico inverso $\mathbb{G}^{-1}$ puede calcularse por cofactores

$$
\mathbb{G}^{-1}=\frac{1}{g}[\operatorname{Cof}(\mathbb{G})]^{T}
$$

de (6.6) podemos ver que $\mathbb{G}$ es simétrico, por lo que podemos escribir

$$
\mathbb{G}^{-1}=\frac{1}{g} \operatorname{Cof}(\mathbb{G}) \text {. }
$$

El cofactor del elemento $g_{i j}$ de $\mathbb{G}$ es el valor del determinante que resulta de $\mathbb{G}$ al eliminar el $i$-ésimo renglón y la $j$-ésima columna a la que pertenece $g_{i j}$ y multiplicado por $(-1)^{i+j}$. De este modo tenemos

$$
\operatorname{Cof}(\mathbb{G})_{i j}=(-1)^{i+j} \operatorname{det}_{i j} \mathbb{G} \equiv G(i, j)
$$

entonces

$$
g^{i j} \equiv\left(\mathbb{G}^{-1}\right)_{i j}=\frac{1}{g} G(i, j) .
$$

Por otro lado, $g=\operatorname{det}(\mathbb{G})$ se puede calcular también por cofactores

$$
g=g_{11} G(1,1)+g_{12} G(1,2)+g_{13} G(1,3),
$$

en forma compacta

$$
g=\sum_{j} g_{i j} G(i, j)
$$

esto significa que el determinante es una combinación lineal de los elementos $g_{i j}$ y dado que $G(i, j)$ no contiene al elemento $g_{i j}$ entonces

$$
\frac{\partial g}{\partial g_{i j}}=G(i, j)=g g^{i j}
$$


Por otra parte, para obtener la relación entre los símbolos de Christoffel y el tensor métrico $\mathbb{G}$ recordemos la definición

$$
g_{i j}=\frac{\partial x^{l}}{\partial y^{i}} \frac{\partial x^{l}}{\partial y^{j}}
$$

y calculemos la derivada de $g_{i j}$ con respecto a la coordenada $y_{k}$

$$
\frac{\partial g_{i j}}{\partial y^{k}}=\frac{\partial^{2} x^{l}}{\partial y^{k} \partial y^{i}} \frac{\partial x^{l}}{\partial y^{j}}+\frac{\partial^{2} x^{l}}{\partial y^{k} \partial y^{j}} \frac{\partial x^{l}}{\partial y^{i}} .
$$

De la definición de los símbolos de Christoffel obtenemos

$$
\frac{\partial g_{i j}}{\partial y^{k}}=\Gamma_{k i}^{m} \frac{\partial x^{l}}{\partial y^{m}} \frac{\partial x^{l}}{\partial y^{j}}+\Gamma_{k j}^{m} \frac{\partial x^{l}}{\partial y^{m}} \frac{\partial x^{l}}{\partial y^{i}}=g_{m j} \Gamma_{k i}^{m}+g_{m i} \Gamma_{k j}^{m} .
$$

Por otro lado, usando (6.17) y la regla de la cadena obtenemos la relación buscada entre los símbolos de Christoffel y el tensor métrico

$$
\frac{\partial g}{\partial y^{k}}=\frac{\partial g}{\partial g_{i j}} \frac{\partial g_{i j}}{\partial y^{k}}=G(i, j)\left[g_{m j} \Gamma_{k i}^{m}+g_{m i} \Gamma_{k j}^{m}\right]=g g^{i j}\left[g_{m j} \Gamma_{k i}^{m}+g_{m i} \Gamma_{k j}^{m}\right]=g\left[\delta_{m}^{i} \Gamma_{k i}^{m}+\delta_{m}^{j} \Gamma_{k j}^{m}\right]=2 g \Gamma_{m k}^{m}
$$

Despejando $\Gamma_{m k}^{m}$

$$
\Gamma_{m k}^{m}=\frac{1}{2 g} \frac{\partial g}{\partial y^{k}}=\frac{1}{2} \frac{\partial \ln g}{\partial y^{k}}=\frac{\partial \ln \sqrt{g}}{\partial y^{k}}=\frac{1}{\sqrt{g}} \frac{\partial \sqrt{g}}{\partial y^{k}}
$$

llegamos a

$$
\Gamma_{m k}^{m}=\frac{1}{\sqrt{g}} \frac{\partial \sqrt{g}}{\partial y^{k}}
$$

Sustituyendo en (6.16) obtenemos la forma generalizada de $\nabla \cdot A=0$

$$
\frac{1}{\sqrt{g}} \frac{\partial \sqrt{g}}{\partial y^{l}} A^{l}(y)+\frac{\partial A^{l}(y)}{\partial y^{l}}=0
$$

o bien

$$
\frac{1}{\sqrt{g}} \frac{\partial}{\partial y^{l}}\left[\sqrt{g} A^{l}(y)\right]=0 .
$$

Para $A^{l}(y)=\rho(y) v^{l}(y)$ obtenemos la forma generalizada de la ecuación de continuidad profunda

$$
\frac{1}{\sqrt{g}} \frac{\partial}{\partial y^{l}}\left[\sqrt{g} \rho(y) v^{l}(y)\right]=0
$$

\subsection{Cálculo del campo ajustado $\mathrm{v}$ en coordenadas generalizadas}

En meteorología, el procedimiento seguido para simplificar las condiciones de frontera impuestas por la topografía es transformar las ecuaciones a un sistema de coordenadas que "siguen el terreno" llamado sistema de coordenadas $\sigma$ [35]. En esta sección damos las expresiones para calcular el campo ajustado $\mathbf{v}$ en coordenadas arbitrarias $y^{1}, y^{2}, y^{3}$ definidas por medio de un conjunto de transformaciones

$$
x^{i}=x^{i}(y) \quad i=1,2,3,
$$

con matriz Jacobiana $\mathbb{J}_{i j}=\partial x^{i} / \partial y^{j}$. Sean $\mathcal{D}_{y}, \Gamma_{y}, \Gamma_{y D}, \Gamma_{y N}$ la preimagen de $\mathcal{D}, \Gamma, \Gamma_{D}, \Gamma_{N}$, respectivamente, bajo (6.20) y sea $\sqrt{g}=|\operatorname{det}(\mathbb{J})|$. Considérese la matriz $\mathbb{S}(x)$ descrita en el capítulo 4.

Comencemos con la transformación del funcional

$$
J(\mathbf{w})=\left\|\mathbf{w}-\mathbf{v}^{0}\right\|_{\mathcal{D} S}=\int_{\mathcal{D}}\left\|\mathbf{w}-\mathbf{v}^{0}\right\|_{S}^{2} d x
$$

donde $\|\cdot\|_{S}$ es la norma asociada al producto escalar $\langle\mathbf{w} \mid \mathbf{u}\rangle_{S}=\mathbf{w}^{t} \mathbb{S} \mathbf{u}$, y $\mathbf{w}, \mathbf{u}$ son vectores columna con componentes cartesianas. Si $\mathbf{u}^{\eta}$ es el vector definido por las componentes contravariantes $A^{k}(y)$ de $\mathbf{u}$, la ley 
de trasformación contravariante $A^{i}(x)=\partial x^{i} / \partial y^{k} A^{k}(y)$ tiene la forma $\mathbf{u}=\mathbb{J} \mathbf{u}^{\eta}$. Entonces $\mathbf{w}^{t}=\left(\mathbf{w}^{\eta}\right)^{t} \mathbb{J}^{t} \mathrm{y}$ $\langle\mathbf{w} \mid \mathbf{u}\rangle_{S}$ tienen la forma

$$
\langle\mathbf{w} \mid \mathbf{u}\rangle_{S}=\left(\mathbf{w}^{\eta}\right)^{t} \mathbb{M} \mathbf{u}^{\eta}
$$

donde $\mathbb{M} \equiv \mathbb{J}^{t} \mathbb{S} J$ conserva las propiedades de $\mathbb{S}$. Entonces $\langle\mathbf{w} \mid \mathbf{u}\rangle_{M} \equiv\left(\mathbf{w}^{\eta}\right)^{t} \mathbb{M} \mathbf{u}^{\eta}$ es un producto escalar y la expresión

$$
\langle\mathbf{w} \mid \mathbf{u}\rangle_{\mathcal{D} M}=\int_{\mathcal{D}_{y}}\left(\mathbf{w}^{\eta}\right)^{t} \mathbb{M} \mathbf{u}^{\eta} \sqrt{g} d y
$$

define un producto interior en el espacio $\mathbf{L}^{2}\left(\mathcal{D}_{y}\right)=\left\{W^{i=1,2,3}(y) \in L_{g}^{2}\left(\mathcal{D}_{y}\right)\right\}$ donde $L_{g}^{2}\left(\mathcal{D}_{y}\right)$ tiene el producto interior $\langle f, q\rangle=\int_{\mathcal{D}_{y}} f q \sqrt{g} d y$. Sea $\|\cdot\|_{\mathcal{D} M}$ la norma asociada a $\langle\cdot \mid \cdot\rangle_{\mathcal{D} M}$. De acuerdo con la identidad $\|\mathbf{u}\|_{\mathcal{D} S}^{2}=$ $\|\mathbf{u}\|_{\mathcal{D} M}^{2}$ la forma contravariante de $J(\cdot)$ es

$$
J(\mathbf{w})=\left\|\mathbf{w}-\mathbf{v}^{0}\right\|_{\mathcal{D} M}^{2}
$$

Sea $\mathbf{n}_{\tau}=\left(n_{1}, n_{2}, n_{3}\right)^{t}$ el vector columna definido por las componentes covariantes de $\mathbf{n}$, entonces $\mathbf{w} \cdot \mathbf{n}=\mathbf{n}_{\tau}^{t} \mathbf{w}^{\eta}$ y el espacio $\mathbb{V}$ toma la forma

$$
\mathbb{V}_{y}=\left\{\mathbf{w}^{\eta}: \nabla \cdot \mathbf{w}=0 \text { en } \mathcal{D}_{y}, \mathbf{n}_{\tau}^{t} \mathbf{w}^{\eta}=0 \text { sobre } \Gamma_{N y}\right\}
$$

donde

$$
\nabla \cdot \mathbf{w}=g^{-1 / 2} \nabla_{y}^{t} g^{1 / 2} \mathbf{w}^{\eta}
$$

y $\nabla_{y}=\left(\partial_{y^{1}}, \partial_{y^{2}}, \partial_{y^{3}}\right)^{t}$. El complemento ortogonal de $\mathbb{V}_{y}$ en $\mathbf{L}_{g}^{2}(\mathcal{D})$ es $\mathbb{V}{ }_{y}^{\perp}=\left\{\nabla_{y} q: q \in H_{D}^{1}\left(\mathcal{D}_{y}\right)\right\}$ donde $H_{D}^{1}\left(\mathcal{D}_{y}\right)$ es el conjunto de funciones en $H^{1}\left(\mathcal{D}_{y}\right)$ que satisfacen $\left.q\right|_{\Gamma_{D y}}=0$. Entonces la minimización de $(6.22)$ en $\mathbb{V}_{y}$ nos proporciona a $\mathbb{M}\left(\mathbf{v}^{\eta}-\mathbf{v}^{0, \eta}\right)-\nabla_{y} \lambda=0 \operatorname{con} \lambda \in H_{D}^{1}\left(\mathcal{D}_{y}\right)$ y

$$
\mathbf{v}^{\eta}=\mathbf{v}^{0, \eta}+\mathbb{M}^{-1} \nabla_{y} \lambda
$$

La condición $\mathbf{n}_{\tau}^{t} \mathbf{v}^{\eta}=0$ en $\Gamma_{N y}$ es dada en términos de las componentes covariantes $n_{l}$ de $\mathbf{n}$ pero existe $c(y)$ tal que $n_{l}=c(y) n_{y l}$ donde $n_{y l}$ son las componentes del vector normal unitario $\mathbf{n}_{y}$ a $\Gamma_{y}$, por lo que la condición toma la forma

$$
\mathbf{n}_{y}^{t} \mathbf{v}^{\eta}=0 \text { sobre } \Gamma_{N y} .
$$

La sustitución de (6.24) en la forma (6.23) da como resultado la ecuación

$$
L_{y} \lambda=\sqrt{g} \nabla \cdot \mathbf{v}^{0} \quad \text { en } \mathcal{D}_{y}
$$

donde $L_{y} \equiv-\nabla_{y}^{t} \sqrt{g} \mathbb{M}^{-1} \nabla_{y}$, sujeta a

$$
\lambda=0 \text { sobre } \Gamma_{D y} .
$$

El teorema de Lax-Milgram garantiza la existencia y unicidad de $\lambda$. Las ecuaciones (6.20) son suaves y en consecuencia, $\lambda(y)$ y $\mathbf{v}^{\eta}$ también. En consecuencia, podemos poner (6.24) en (6.25) para obtener la forma generalizada de las CFN,

$$
\mathcal{L}_{y} \lambda=-\mathbf{n}_{y}^{t} \mathbf{v}^{0} \quad \text { sobre } \Gamma_{N y}
$$

donde $\mathcal{L}_{y} \equiv \mathbf{n}_{y}^{t} \mathbb{M}^{-1} \nabla_{y}$. Las ecuaciones (6.24) nos dan la forma contravariante de $\mathbf{v}$, usando $\mathbf{v}=\mathbb{J} \mathbf{v}^{\eta}$ obtenemos la forma cartesiana

$$
\mathbf{v}=\mathbf{v}^{0}+\mathbb{S}^{-1}\left(\mathbb{J}^{t}\right)^{-1} \nabla_{y} \lambda
$$

La forma usual de las ecuaciones (6.1) mantienen las coordenadas horizontales $\left(y^{1}=x, y^{2}=y\right)$ e introducen la coordenada vertical

$$
\sigma=y^{3}(x, y, z)
$$

que es constante sobre la topografía: $\sigma[x, y, z=h(x, y)] \equiv \sigma_{h}$ sobre $\Gamma_{N}$. De (6.30) tenemos $z=z(x, y, \sigma)$, $\mathbb{J}_{i j}=\mathbb{J}_{i j}^{-1}=\delta_{i j}$ para $i=1,2, j=1,2,3$,

$$
\mathbb{J}_{3 j}=\left(\begin{array}{lll}
z_{x} & z_{y} & z_{\sigma}
\end{array}\right), \quad \quad \mathbb{J}_{3 j}^{-1}=\left(\begin{array}{lll}
-z_{x} / z_{\sigma} & -z_{y} / z_{\sigma} & 1 / z_{\sigma}
\end{array}\right),
$$


donde $\sqrt{g} \equiv z_{\sigma}$ y los subíndices significan derivación, e.g. $\partial_{x} z \equiv z_{x}$. Todo esto, junto con $\mathbb{S}_{k l}^{-1}=\delta_{k l} \alpha_{k}^{-2}$ nos proporcionan a $M_{12}^{-1}=0, M_{i i}^{-1}=\alpha_{i}^{-2}(i=1,2), M_{13}^{-1}=-z_{x} / z_{\sigma} \alpha_{1}^{2}, M_{23}^{-1}=-z_{y} / z_{\sigma} \alpha_{2}^{2}$,

$$
M_{33}^{-1}=\left(\frac{z_{x}}{z_{\sigma} \alpha_{1}}\right)^{2}+\left(\frac{z_{y}}{z_{\sigma} \alpha_{2}}\right)^{2}+\left(\frac{1}{z_{\sigma} \alpha_{3}}\right)^{2}
$$

Si $u^{0}, v^{0}, w^{0}$ son las componentes cartesianas de $\mathbf{v}^{0}$, las componentes contravariantes $\operatorname{son} V^{0,1}(y)=u^{0}$, $V^{0,2}(y)=v^{0}$,

$$
V^{0,3}(y)=\frac{-z_{x} u^{0}-z_{y} v^{0}+w^{0}}{z_{\sigma}}
$$

relaciones similares se observan para las componentes de $\mathbf{v}$. Para las ecuaciones (6.7) tenemos

$$
\begin{aligned}
L_{y} & =-\partial_{x} \frac{1}{\alpha_{1}^{2}}\left(z_{\sigma} \partial_{x}-z_{x} \partial_{\sigma}\right)-\partial_{y} \frac{1}{\alpha_{2}^{2}}\left(z_{\sigma} \partial_{y}-z_{y} \partial_{\sigma}\right)-\partial_{\sigma}\left\{-\frac{z_{x}}{\alpha_{1}^{2}} \partial_{x}-\frac{z_{y}}{\alpha_{2}^{2}} \partial_{y}+z_{\sigma} M_{33}^{-1} \partial_{\sigma}\right\} \\
\sqrt{g} \nabla \cdot \mathbf{v}^{0} & =\partial_{x} z_{\sigma} u^{0}+\partial_{y} z_{\sigma} v^{0}+\partial_{\sigma} z_{\sigma} V^{0,3}(y) .
\end{aligned}
$$

Usando $n_{y k}=-\delta_{3 k}$ la CFN (6.28) sobre $\Gamma_{N y}=\left\{\left(y^{1}, y^{2}, y^{3}=\sigma_{h}\right)\right\}$ toman la forma

$$
\frac{z_{x}}{z_{\sigma} \alpha_{1}^{2}} \lambda_{x}+\frac{z_{y}}{z_{\sigma} \alpha_{2}^{2}} \lambda_{y}-M_{33}^{-1} \lambda_{\sigma}=V^{3,0}(y)
$$

\subsection{Expresiones con coordenadas $\sigma$ usadas en la literatura}

Los ejemplos de coordenadas $\sigma$ definidas al principio de este capítulo tienen la forma

$$
y^{1}=x \quad y^{2}=y \quad y^{3}=y^{3}(x, y, z)
$$

y

$$
x=y^{1} \quad y=y^{2} \quad z=z\left(y^{1}, y^{3}, y^{2}\right)
$$

donde la coordenada $\sigma$ toma un valor constante tanto en la superficie terrestre,

$$
\sigma[x, y, z=h(x, y)]=\text { cte } \quad \text { sobre } \Gamma_{N},
$$

como en la frontera superior $z=z_{\max }$,

$$
\sigma\left[x, y, z=z_{\max }\right]=\text { cte } \quad \text { sobre } \Gamma_{D} .
$$

Las coordenadas $x, y$ están acotadas por valores constantes. Así, en el espacio físico el dominio $\mathcal{D}$ tiene la forma

$$
\mathcal{D}=\left\{(x, y, z): x_{\min } \leq x \leq x_{\max } \quad y_{\min } \leq y \leq y_{\max } \quad h(x, y) \leq z \leq z_{\max }\right\}
$$

y su imagen bajo la transformación $y^{k}=y^{k}(x)$ es un paralelepípedo

$$
\mathcal{D}_{y}=\left\{\left(y^{1}, y^{2}, y^{3}\right): x_{\min } \leq y^{1} \leq x_{\max } \quad y_{\min } \leq y^{2} \leq y_{\max } \quad \sigma_{\min } \leq y^{3} \leq \sigma_{\max }\right\}
$$

En estos casos la forma explícita de $\mathbb{J}, \mathbb{J}^{-1}, \mathbb{G}, \mathbb{G}^{-1}, \nabla \cdot \mathbf{v}=0, L_{y}$ y $\mathcal{L}_{y}$ es como sigue.

Las matrices $\mathbb{J}, \mathbb{J}^{-1}, \mathbb{G}, \mathbb{G}^{-1}$ asociadas a la transformación (6.37) están dadas por

$$
\begin{aligned}
\mathbb{J}=\left(\begin{array}{ccc}
1 & 0 & 0 \\
0 & 1 & 0 \\
z_{1} & z_{2} & z_{3}
\end{array}\right) & \mathbb{J}^{-1}=\left(\begin{array}{ccc}
1 & 0 & 0 \\
0 & 1 & 0 \\
-z_{1} / z_{3} & -z_{2} / z_{3} & 1 / z_{3}
\end{array}\right) \\
\mathbb{G}=\left(\begin{array}{cccc}
1+z_{1}^{2} & z_{1} z_{2} & z_{1} z_{3} \\
z_{1} z_{2} & 1+z_{2}^{2} & z_{2} z_{3} \\
z_{1} z_{3} & z_{2} z_{3} & z_{3}^{2}
\end{array}\right) & \mathbb{G}^{-1}=\left(\begin{array}{ccc}
1 & 0 & -z_{1} / z_{3} \\
0 & 1 & -z_{2} / z_{3} \\
-z_{1} / z_{3} & -z_{2} / z_{3} & \left(z_{1}^{2}+z_{2}^{2}+1\right) / z_{3}^{2}
\end{array}\right)
\end{aligned}
$$


donde usamos la notación

$$
z_{j} \equiv \frac{\partial z}{\partial y^{j}}, \quad J=\sqrt{g}=z_{3}=\frac{\partial z}{\partial y^{3}}
$$

2. Tenemos

$$
\begin{aligned}
\mathbb{M} & =\left[\begin{array}{lll}
1 & 0 & z_{1} \\
0 & 1 & z_{2} \\
0 & 0 & z_{3}
\end{array}\right]\left[\begin{array}{ccc}
\alpha_{1}^{2} & 0 & 0 \\
0 & \alpha_{2}^{2} & 0 \\
0 & 0 & \alpha_{3}^{2}
\end{array}\right]\left[\begin{array}{ccc}
1 & 0 & 0 \\
0 & 1 & 0 \\
z_{1} & z_{2} & z_{3}
\end{array}\right] \\
& =\left[\begin{array}{lll}
1 & 0 & z_{1} \\
0 & 1 & z_{2} \\
0 & 0 & z_{3}
\end{array}\right]\left[\begin{array}{ccc}
\alpha_{1}^{2} & 0 & 0 \\
0 & \alpha_{2}^{2} & 0 \\
\alpha_{3}^{2} z_{1} & \alpha_{3}^{2} z_{2} & \alpha_{3}^{2} z_{3}
\end{array}\right] \\
& =\left[\begin{array}{ccc}
\alpha_{1}^{2}+z_{1}^{2} \alpha_{3}^{2} & z_{1} z_{2} \alpha_{3}^{2} & z_{1} z_{3} \alpha_{3}^{2} \\
z_{1} z_{2} \alpha_{3}^{2} & \alpha_{2}^{2}+z_{2}^{2} \alpha_{3}^{2} & z_{2} z_{3} \alpha_{3}^{2} \\
z_{1} z_{3} \alpha_{3}^{2} & z_{2} z_{3} \alpha_{3}^{2} & z_{3}^{2} \alpha_{3}^{2}
\end{array}\right] .
\end{aligned}
$$

Para el cálculo de $\mathbb{M}^{-1}=\left(\mathbb{J}^{t} \tilde{\alpha}^{2} \mathbb{J}\right)^{-1}=\mathbb{J}^{-1}\left(\mathbb{J}^{t} \tilde{\alpha}^{2}\right)^{-1}=\mathbb{J}^{-1}\left[\left(\tilde{\alpha}^{2} \mathbb{J}\right)^{-1}\right]^{t}$ tenemos

$$
\tilde{\alpha}^{2} \mathbb{J}=\left[\begin{array}{ccc}
\alpha_{1}^{2} & 0 & 0 \\
0 & \alpha_{2}^{2} & 0 \\
z_{1} \alpha_{3}^{2} & z_{2} \alpha_{3}^{2} & z_{3} \alpha_{3}^{2}
\end{array}\right] \quad\left(\tilde{\alpha}^{2} \mathbb{J}\right)^{-1}=\left[\begin{array}{ccc}
1 / \alpha_{1}^{2} & 0 & 0 \\
0 & 1 / \alpha_{2}^{2} & 0 \\
-z_{1} / z_{3} \alpha_{1}^{2} & -z_{2} / z_{3} \alpha_{2}^{2} & 1 / z_{3} \alpha_{3}^{2}
\end{array}\right]
$$

y por tanto

$$
\mathbb{M}^{-1}=\left[\begin{array}{ccc}
1 & 0 & 0 \\
0 & 1 & 0 \\
-\frac{z_{1}}{z_{3}} & -\frac{z_{2}}{z_{3}} & \frac{1}{z_{3}}
\end{array}\right]\left[\begin{array}{ccc}
\frac{1}{\alpha_{1}^{2}} & 0 & -\frac{z_{1}}{z_{3} \alpha_{1}^{2}} \\
0 & \frac{1}{\alpha_{2}^{2}} & -\frac{z_{2}}{z_{3} \alpha_{2}^{2}} \\
0 & 0 & \frac{1}{z_{3} \alpha_{3}^{2}}
\end{array}\right]=\left[\begin{array}{ccc}
\frac{1}{\alpha_{1}^{2}} & 0 & -\frac{z_{1}}{z_{3} \alpha_{1}^{2}} \\
0 & \frac{1}{\alpha_{2}^{2}} & -\frac{z_{2}}{z_{3} \alpha_{2}^{2}} \\
-\frac{z_{1}}{z_{3} \alpha_{1}^{2}} & -\frac{z_{2}}{z_{3} \alpha_{2}^{2}} & M_{33}^{-1}
\end{array}\right]
$$

con

$$
M_{33}^{-1}=\left(\frac{z_{1}}{z_{3} \alpha_{1}}\right)^{2}+\left(\frac{z_{2}}{z_{3} \alpha_{2}}\right)^{2}+\left(\frac{1}{z_{3} \alpha_{3}}\right)^{2} .
$$

3. La notación estándar para las componentes cartesianas del campo inicial $\mathbf{v}^{0}$ es

$$
v^{1,0}(x)=u^{0} \quad v^{2,0}(x)=v^{0} \quad v^{3,0}(x)=w^{0} \quad \text { ó } \quad \mathbf{v}^{0}=\hat{\mathbf{x}}_{i} v^{i, 0}(x)=\hat{\mathbf{x}} u^{0}+\hat{\mathbf{y}} v^{0}+\hat{\mathbf{z}} w^{0} .
$$

Las componentes contravariantes de $\mathbf{v}^{0}$ son

$$
\left(\begin{array}{c}
v^{1,0}(y) \\
v^{2,0}(y) \\
v^{3,0}(y)
\end{array}\right)=\mathbb{J}^{-1}\left(\begin{array}{c}
u^{0}(x) \\
v^{0}(x) \\
w^{0}(x)
\end{array}\right)=\left(\begin{array}{c}
u^{0} \\
v^{0} \\
\frac{-z_{1} u^{0}-z_{2} v^{0}+w^{0}}{z_{3}}
\end{array}\right)
$$

En forma análoga, las componentes contravariantes del campo ajustado son

$$
\left(\begin{array}{c}
v^{1}(y) \\
v^{2}(y) \\
v^{3}(y)
\end{array}\right)=\left(\begin{array}{c}
u \\
v \\
\frac{-z_{1} u-z_{2} v+w}{z_{3}}
\end{array}\right)
$$

4. La ecuación de continuidad $\nabla \cdot \mathbf{v}=0$ tiene la forma

$$
\frac{\partial}{\partial y^{1}} z_{3} v^{1}+\frac{\partial}{\partial y^{2}} z_{3} v^{2}+\frac{\partial}{\partial y^{3}} z_{3} v^{3}=0
$$


5. En la ecuación elíptica $L_{y} \lambda=\sqrt{g} \nabla \cdot \mathbf{v}^{0}$ tenemos

$$
\begin{aligned}
& L_{y}=-\nabla^{t} z_{3} \mathbb{M}^{-1} \nabla=-\left(\partial_{1} \partial_{2} \partial_{3}\right)\left[\begin{array}{ccc}
\frac{z_{3}}{\alpha_{1}^{2}} & 0 & -\frac{z_{1}}{\alpha_{1}^{2}} \\
0 & \frac{z_{3}}{\alpha_{2}^{2}} & -\frac{z_{2}^{2}}{\alpha_{2}^{2}} \\
-\frac{z_{1}}{\alpha_{1}^{2}} & -\frac{z_{2}}{\alpha_{2}^{2}} & z_{3} M_{33}^{-1}
\end{array}\right]\left(\begin{array}{l}
\partial_{1} \\
\partial_{2} \\
\partial_{3}
\end{array}\right) \\
& =-\left(\begin{array}{lll}
\partial_{1} & \partial_{2} \partial_{3}
\end{array}\right)\left[\begin{array}{c}
\frac{z_{3}}{\alpha_{1}^{2}} \partial_{1}-\frac{z_{1}}{\alpha_{1}^{2}} \partial_{3} \\
\frac{z_{3}^{3}}{\alpha_{2}^{2}} \partial_{2}-\frac{z_{2}^{2}}{\alpha_{2}^{2}} \partial_{3} \\
-\frac{z_{1}}{\alpha_{1}^{2}} \partial_{1}-\frac{z_{2}}{\alpha_{2}^{2}} \partial_{2}+z_{3} M_{33}^{-1} \partial_{3}
\end{array}\right]
\end{aligned}
$$

Ó

$$
-L_{y}=\partial_{1} \frac{1}{\alpha_{1}^{2}}\left(z_{3} \partial_{1}-z_{1} \partial_{3}\right)+\partial_{2} \frac{1}{\alpha^{2}}\left(z_{3} \partial_{2}-z_{2} \partial_{3}\right)+\partial_{3}\left\{-\frac{z_{1}}{\alpha_{1}^{2}} \partial_{1}-\frac{z_{2}}{\alpha_{2}^{2}} \partial_{2}+z_{3} M_{33}^{-1} \partial_{3}\right\},
$$

donde

$$
z_{3} M_{33}^{-1}=\frac{1}{z_{3}}\left[\left(\frac{z_{1}}{\alpha_{1}}\right)^{2}+\left(\frac{z_{2}}{\alpha_{2}}\right)^{2}+\left(\frac{1}{\alpha_{3}}\right)^{2}\right]
$$

y

$$
\sqrt{g} \nabla \cdot \mathbf{v}^{0}=\frac{\partial}{\partial y^{1}} z_{3} u^{0}+\frac{\partial}{\partial y^{2}} z_{3} v^{0}+\frac{\partial}{\partial y^{3}}\left(-z_{1} u^{0}-z_{2} v^{0}+w^{0}\right) .
$$

La condición de frontera Dirichlet $\lambda=0$ en $\Gamma_{D y}$ equivale las condiciones

$$
\begin{aligned}
& \lambda\left(y^{1}=x_{\text {mín }}, y^{2}, y^{3}\right)=0, \\
& \lambda\left(y^{1}=x_{\text {máx }}, y^{2}, y^{3}\right)=0, \\
& \lambda\left(y^{1}, y^{2}=y_{\text {mín }}, y^{3}\right)=0, \\
& \lambda\left(y^{1}, y^{2}=y_{\text {máx }}, y^{3}\right)=0, \\
& \lambda\left(y^{1}, y^{2}, y^{3}=z_{\text {máx }}\right)=0 .
\end{aligned}
$$

Para la condición de frontera Neumann $\mathcal{L}_{y} \lambda=-\hat{\mathbf{n}}_{y}^{t} \mathbf{v}^{0}$ sobre $\Gamma_{N y}=\left\{\left(y^{1}, y^{2}, y^{3}=\sigma_{\min }\right)\right\}$ tenemos $\hat{\mathbf{n}}_{y}=$ $\hat{\mathbf{y}}_{l} \hat{n}_{y l}=-\hat{\mathbf{y}}_{3}$, por tanto

$$
\begin{aligned}
& \mathcal{L}_{y}=\hat{\mathbf{n}}_{y}^{t} \mathbb{M}^{-1} \nabla=\left(\begin{array}{ccc}
0, & 0, & -1
\end{array}\right)\left[\begin{array}{ccc}
\frac{1}{\alpha_{1}^{2}} & 0 & -\frac{z_{1}}{z_{3} \alpha_{1}^{2}} \\
0 & \frac{1}{\alpha_{2}^{2}} & -\frac{z_{2}}{z_{3} \alpha_{2}^{2}} \\
-\frac{z_{1}}{z_{3} \alpha_{1}^{2}} & -\frac{z_{2}}{z_{3} \alpha_{2}^{2}} & M_{33}^{-1}
\end{array}\right]\left(\begin{array}{c}
\partial_{1} \\
\partial_{2} \\
\partial_{3}
\end{array}\right) \\
& =\left(\begin{array}{cc}
\frac{z_{1}}{z_{3} \alpha_{1}^{2}}, & \frac{z_{2}}{z_{3} \alpha_{2}^{2}}, \quad-M_{33}^{-1}
\end{array}\right)\left(\begin{array}{l}
\partial_{1} \\
\partial_{2} \\
\partial_{3}
\end{array}\right) \\
& =\frac{z_{1}}{z_{3} \alpha_{1}^{2}} \partial_{1}+\frac{z_{2}}{z_{3} \alpha_{2}^{2}} \partial_{2}-M_{33}^{-1} \partial_{3}
\end{aligned}
$$

$\mathrm{y}$

$$
\hat{\mathbf{n}}_{y}^{t} \mathbf{v}^{0}=-v^{3,0}
$$

Así $\mathcal{L}_{y} \lambda=-\hat{\mathbf{n}}_{y}^{t} \mathbf{v}^{0}$ queda como sigue

$$
\left(\frac{z_{1}}{z_{3} \alpha_{1}^{2}} \partial_{1}+\frac{z_{2}}{z_{3} \alpha_{2}^{2}} \partial_{2}-M_{33}^{-1} \partial_{3}\right) \lambda=v^{3,0}(y) \quad \text { sobre } \mathcal{D}
$$


6. Las componentes contravariantes del campo ajustado están dadas por

$$
\left(\begin{array}{l}
v^{1}(y) \\
v^{2}(y) \\
v^{3}(y)
\end{array}\right)=\left(\begin{array}{l}
v^{1,0}(y) \\
v^{2,0}(y) \\
v^{3,0}(y)
\end{array}\right)+\left[\begin{array}{ccc}
\frac{1}{\alpha_{1}^{2}} & 0 & -\frac{z_{1}}{z_{3} \alpha_{1}^{2}} \\
0 & \frac{1}{\alpha_{2}^{2}} & -\frac{z_{2}}{z_{3} \alpha_{2}^{2}} \\
-\frac{z_{1}}{z_{3} \alpha_{1}^{2}} & -\frac{z_{2}}{z_{3} \alpha_{2}^{2}} & M_{33}^{-1}
\end{array}\right]\left(\begin{array}{c}
\partial_{1} \lambda \\
\partial_{2} \lambda \\
\partial_{3} \lambda
\end{array}\right)
$$

y las componentes cartesianas por

$$
\left(\begin{array}{l}
v^{1}(x) \\
v^{2}(x) \\
v^{3}(x)
\end{array}\right)=\left(\begin{array}{l}
v^{1,0}(x) \\
v^{2,0}(x) \\
v^{3,0}(x)
\end{array}\right)+\left[\begin{array}{ccc}
\frac{1}{\alpha_{1}^{2}} & 0 & -\frac{z_{1}}{z_{3} \alpha_{1}^{2}} \\
0 & \frac{1}{\alpha_{2}^{2}} & -\frac{z_{2}}{z_{3} \alpha_{2}^{2}} \\
0 & 0 & \frac{1}{z_{3} \alpha_{3}^{2}}
\end{array}\right]\left(\begin{array}{l}
\partial_{1} \lambda \\
\partial_{2} \lambda \\
\partial_{3} \lambda
\end{array}\right)
$$

\subsection{Formulación variacional del problema elíptico $L_{y} \lambda=f$}

En la sección 6.4 consideramos la formulación variacional del problema elíptico (6.26), (6.27), (6.28). La solución $\lambda$ pertenece al espacio $L_{2}\left(\mathcal{D}_{y}\right)=\left\{v: \int_{\mathcal{D}_{y}}|v|^{2} d y<\infty\right\}$ con el producto interior

$$
\langle v \mid u\rangle_{y} \equiv \int_{\mathcal{D}_{y}} v u d y
$$

y la norma $\|v\|_{y}=\langle v \mid v\rangle_{y}^{1 / 2}$. Mas aún, $\lambda$ pertenece al espacio $V_{a}=\left\{v \in H^{1}\left(\mathcal{D}_{y}\right): v=0\right.$ sobre $\left.\Gamma_{D y}\right\}$. Para obtener la formulación variacional (o débil) del problema elíptico (6.26-6.28) multiplicamos (6.26) por una función $v$ en $V_{a}$ e integramos,

$$
\left\langle L_{y} \lambda \mid v\right\rangle_{y}=\langle f \mid v\rangle_{y} \quad \text { para cada } v \in V_{a}
$$

El lado izquierdo se integra usando el teorema de Green en el espacio:

$$
\left\langle L_{y} \lambda \mid v\right\rangle_{y}=-\oint_{\Gamma_{y}} \sqrt{g} v \mathcal{L}_{y} \lambda d s_{y}+a_{y}(\lambda, v),
$$

donde $\hat{n}_{y k}$ son las componentes del vector normal, unitario y exterior a $\Gamma_{y}$, y aparece el operador

$$
\mathcal{L}_{y}=\sum_{k l} \hat{n}_{y k} M_{k l}^{-1} \frac{\partial}{\partial y^{l}}
$$

y la forma bilineal

$$
a_{y}(u, v) \equiv \sum_{k l}\left\langle\sqrt{g} M_{k l}^{-1} \frac{\partial u}{\partial y^{l}}, \frac{\partial v}{\partial y^{k}}\right\rangle_{y}=\int_{\mathcal{D}_{y}}(\nabla u)^{t} \mathbb{M}^{-1} \nabla v \sqrt{g} d y
$$

Entonces (6.50) queda como sigue

$$
a_{y}(\lambda, v)=\langle f \mid v\rangle_{y}+\oint_{\Gamma_{y}} \sqrt{g} v \mathcal{L}_{y} \lambda d s_{y} \quad \text { para cada } v \in V_{a} .
$$

Dado que $v$ satisface $v=0$ en $\Gamma_{D y}$ y $\mathcal{L}_{y} \lambda=q$ tiene lugar en $\Gamma_{N y}$, la integral de superficie toma la forma

$$
\oint_{\Gamma_{y}} \sqrt{g} v \mathcal{L}_{y} \lambda d s_{y}=\int_{\Gamma_{N y}} \sqrt{g} v q d s_{y} .
$$

Así, concluimos que si $\lambda$ existe debe satisfacer la ecuación integral

$$
a_{y}(\lambda, v)=\tilde{f}_{y}(v) \quad \text { para cada } v \in V_{a}
$$


donde definimos

$$
\tilde{f}_{y}(v) \equiv\langle f \mid v\rangle_{y}+\int_{\Gamma_{N y}} \sqrt{g} v q d s_{y} .
$$

La ecuación (6.51) recibe el nombre de forma débil del problema elíptico (6.26-6.28). Si la transformación de coordenadas $x^{i}=x^{i}(y)$ es bien comportada y los coeficientes $\alpha_{i}^{2}(y)$ no se anulan en $\overline{\mathcal{D}} \equiv \mathcal{D} \cup \Gamma_{y}$, la existencia y unicidad de la solución de la ecuación integral (6.51) está garantizada por las propiedades de $a_{y}(\cdot, \cdot)$ y $\tilde{f}_{y}(\cdot)$.

La simetría de $a_{y}(\cdot, \cdot)$ permite identificar a las soluciones de la ecuación integral (6.51) como las extremales del funcional cuadrático

$$
J_{y}(v)=\frac{1}{2} a_{y}(v, v)-\tilde{f}_{y}(v) \quad \text { para } v \in V_{a} .
$$

En efecto, tenemos

$$
\left.\frac{d}{d \epsilon} J_{y}(u+\epsilon v)\right|_{\epsilon=0}=a_{y}(\lambda, v)-\tilde{f}_{y}(v)=0 \text { para cada } v \in V_{a} .
$$

que es exactamente la ecuación (6.51) y por consiguiente una solución $\lambda$ de (6.51) es una extremal de $J_{y}(v)$. El teorema de Lax-Milgram garantiza que existe una extremal $\lambda$ y es única. Por lo tanto, en lugar de calcular a $\lambda$ por medio de la ecuación integral (6.51), podemos obtenerla como la función que minimiza al funcional $J(\lambda)$. Este último camino tiene la ventaja de incorporar la condición de frontera Neumann $(6.28)$ en $J(\lambda)$ por lo que sólo debemos considerar en forma explícita la condición de frontera Dirichlet (6.26). En la sección 6.4 probamos que si $\lambda$ minimiza a $J_{y}(\lambda)$ satisface $(6.27)$ y, bajo condiciones adicionales de regularidad en los coeficientes de $L_{y}, \lambda$ tiene la propiedad inherente de satisfacer

$$
\mathcal{L}_{y} \lambda=q \quad \text { sobre } \Gamma_{N y} .
$$

\subsubsection{La condición de frontera $\partial \lambda / \partial n=0$ es incorrecta en general}

El procedimiento estándar usado en la literatura para calcular el campo ajustado $\mathbf{V}$ con el funcional

$$
F(\mathbf{W})=\int_{D} \sum_{i} \alpha_{i}^{2}\left(W^{i}-V^{i, 0}\right)^{2} d x
$$

y la condición $\nabla \cdot \mathbf{V}=0$ consiste en minimizar el funcional

$$
J(\mathbf{W}, \lambda)=\int_{D}\left[\sum_{i} \alpha_{i}^{2}\left(W^{i}-V^{i, 0}\right)^{2}+\lambda \nabla \cdot \mathbf{W}\right] d x
$$

donde $\lambda$ es un multiplicador de Lagrange. Debe notarse que ninguna de las referencias consultadas [6-14] considera una definición adecuada del espacio $\mathbb{V}$ al que debe pertenecer tanto el campo ajustado $\mathbf{V}$ como los campos de prueba W. Esta ambigüedad se refleja en las condiciones bajo las cuales se satisface la ecuación

$$
\lambda \delta \mathbf{V} \cdot \hat{\mathbf{n}}=0 \quad \text { sobre } \quad \partial D
$$

la cual se obtiene al minimizar $J(\mathbf{V}+\epsilon \delta \mathbf{V}, \lambda)$. En efecto, (6.52) tiene lugar si $\lambda$ ó $\delta \mathbf{V} \cdot \hat{\mathbf{n}}$ son cero en $\partial D$. Dado que ambas condiciones no pueden imponerse (o el problema esta sobre determinado) la siguiente elección fue adoptada por Christine Sherman [39] y la mayoría de los autores que han usado el esquema variacional:

1. La condición de frontera tipo Dirichlet

$$
\lambda=0
$$

se usa para obtener un campo ajustado $\mathbf{V}$ cuando la frontera $\partial D$ es abierta ó permite un flujo. En tal caso podemos tener $\delta \mathbf{V} \neq 0$ y por tanto $\mathbf{V} \cdot \hat{\mathbf{n}}$ puede diferir de $\mathbf{V}^{0} \cdot \hat{\mathbf{n}}$ sobre $\partial D$.

2. La condición de frontera Neumann homogénea

$$
\frac{\partial \lambda}{\partial n}=0
$$

se usa para "imponer la condición de que el flujo a través de $\partial D$ sea cero" [35]. Se afirma que tal condición es "apropiada" para una frontera cerrada como el terreno $\partial D_{b} \mathrm{y}$, posiblemente, en la frontera superior del dominio $\partial D$. Como veremos enseguida esta interpretación de (6.54) no es correcta. 
Para mostrar que la condición (6.54) es incorrecta en general, consideremos el cálculo del campo ajustado V con el funcional en coordenadas cartesianas

$$
F(\mathbf{W})=\left\|\mathbf{W}-\mathbf{V}^{0}\right\|_{D \alpha}^{2}=\int_{D}\left\langle\mathbf{W}-\mathbf{V}^{0} \mid \mathbf{W}-\mathbf{V}^{0}\right\rangle_{\alpha} d x \quad\left(\langle\mathbf{W} \mid \mathbf{W}\rangle_{\alpha}=\mathbf{W}^{t} \tilde{\alpha}^{2} \mathbf{W}\right)
$$

y la condición de resbalamiento

$$
\mathbf{V} \cdot \hat{\mathbf{n}}=0 \quad \text { sobre } \quad \partial D_{b}
$$

como la única condición de frontera que debe satisfacer el campo ajustado V. El cálculo de $\mathbf{V}$ en el espacio

$$
\mathbb{V}=\left\{\mathbf{W}: \nabla \cdot \mathbf{W}=0 \quad \text { en } \quad D \quad \mathrm{y} \quad \mathbf{W} \cdot \hat{\mathbf{n}}=0 \quad \text { sobre } \quad \partial D_{b}\right\}
$$

da

$$
V^{k}=V^{k, 0}+\frac{1}{\alpha_{k}^{2}} \frac{\partial \lambda}{\partial x^{k}}
$$

y la condición (6.55) da la condición de frontera Neumann no homogénea

$$
\sum_{k} \frac{\hat{n}_{k}}{\alpha_{k}^{2}} \frac{\partial \lambda}{\partial x^{k}}=-\mathbf{V}^{0} \cdot \hat{\mathbf{n}} \quad \text { sobre } \quad \partial D_{b} .
$$

Para apreciar la diferencia con (6.54) reescribamos esta última en la forma

$$
\frac{\partial \lambda}{\partial n}=\nabla \lambda \cdot \hat{\mathbf{n}}=\sum_{k} \hat{n}_{k} \frac{\partial \lambda}{\partial x^{k}}=0 \quad \text { sobre } \quad \partial D_{b} .
$$

Es claro que el único caso en el cual (6.54) coincide con la condición correcta (6.56) es cuando $\alpha_{1}^{2}=\alpha_{2}^{2}=$ $\alpha_{3}^{2}=1 \mathrm{y} \mathbf{V}^{0} \cdot \hat{\mathbf{n}}=0$ sobre $\partial D_{b}$. Desafortunadamente, en la literatura se considera al menos un coeficiente $\alpha_{k}^{2}$ distinto de 1 y la ecuación $\mathbf{V}^{0} \cdot \hat{\mathbf{n}}=0$ no se satisface en general.

De acuerdo con lo anterior, el problema elíptico que se resuelve en la literatura estándar para calcular el multiplicador de Lagrange $\lambda$ tiene la forma

$$
\begin{aligned}
\left(-\sum \frac{\partial}{\partial x^{k}} \frac{1}{\alpha_{k}^{2}} \frac{\partial}{\partial x^{k}}\right) \lambda & =\nabla \cdot \mathbf{V}^{0} \quad \text { en } \quad D \\
\lambda & =0 \text { sobre } \quad \partial D_{a} \\
\frac{\partial \lambda}{\partial n} & =0 \text { sobre } \quad \partial D_{b}
\end{aligned}
$$

donde se usa (6.54) en lugar de la condición correcta (6.56). Veamos algunos ejemplos.

\subsection{Ejemplos reportados en la literatura}

En esta seccion reproducimos los calculos reportados en [31]. Un intento de comparar un MMC contra soluciones exactas de un flujo que pasa sobre topografía simple, es reportado en [38]. El modelo usa las coordenadas

$$
\tilde{x}=x, \quad \tilde{y}=y, \quad \sigma=\left(z_{t}-z\right) / \pi
$$

donde $z_{t}$ es la tapa de $\mathcal{D}, \pi \equiv z_{t}-z_{s}$ y $z_{s}$ denota la elevación del terreno. El funcional $G(\mathbf{v}, \lambda)$ es escrito en términos de $\tilde{x} \tilde{y} \sigma$ y las componentes contravariantes $\tilde{u}, \tilde{v}, \tilde{w}$ de $\mathbf{v}$, cuya relación con las componentes cartesianas $u, v, w$ es la ecuación (6.52): $\tilde{u}=u, \tilde{v}=v \quad \mathrm{y}$

$$
\tilde{w}=-\left(w+\sigma u \pi_{x}+\sigma v \pi_{y}\right) / \pi .
$$

La ecuación elíptica del multiplicador de Lagrange es (6.26) con $\lambda / 2$ en lugar de $\lambda$, y es resuelta con las condiciones de frontera $\lambda \delta \tilde{u}=0, \lambda \delta \tilde{v}=0, \lambda \delta \tilde{w}=0$ sobre las fronteras $x, y, \sigma$ [38]. Los autores afirman que las coordenadas $\tilde{x} \tilde{y} \sigma$ permiten imponer

$$
\tilde{w}_{0}=0
$$


y $\delta \tilde{w}=0$ en $\sigma=1$ (los valores que definen la superficie del terreno $z=z_{s}$ ) donde $\delta \tilde{w} \equiv \tilde{w}-\tilde{w}_{0}$. Entonces $\left.\tilde{w}\right|_{\sigma=1}=0$ y usando (6.58) obtenemos la condición de frontera

$$
w+u \pi_{x}+v \pi_{y}=0 \text { en } \sigma=1 .
$$

Esta condición es inconsistente en general. La CFN natural en la superficie del terreno es (6.54). De (6.57) tenemos $z=z_{t}-\sigma \pi, z_{\sigma}=-\pi, z_{x}=-\sigma \pi_{x}, z_{y}=-\sigma \pi_{y}$ y reemplazando en (6.54) con $\lambda / 2$ en lugar de $\lambda$ tenemos

$$
\frac{\pi_{x} \lambda_{x}}{2 \pi \alpha_{1}^{2}}+\frac{\pi_{y} \lambda_{y}}{2 \pi \alpha_{1}^{2}}-\frac{1}{2} \mathbb{M}_{33}^{-1} \lambda_{\sigma}=\tilde{w}_{0} \quad \text { en } \sigma=1
$$

donde hemos usado la notación de [38] para las $\alpha_{i}\left(\alpha_{1} \equiv \alpha_{1}, \alpha_{2} ; \alpha_{2} \equiv \alpha_{3}\right)$ y

$$
\left.\mathbb{M}_{33}^{-1}\right|_{\sigma=1}=\left(\frac{\pi_{x}}{\pi \alpha_{1}}\right)^{2}+\left(\frac{\pi_{y}}{\pi \alpha_{1}}\right)^{2}+\left(\frac{1}{\pi \alpha_{2}}\right)^{2} .
$$

Para comparar (6.60) con (6.61)se reescriben las ecuaciones (2.a-c) en [38, 786] en términos de $x y \sigma$. El resultado es

$$
\frac{\pi_{x} \lambda_{x}}{2 \alpha_{1}^{2}}+\frac{\pi_{y} \lambda_{y}}{2 \alpha_{1}^{2}}-\left(\frac{1}{2 \alpha_{2}^{2} \pi}+\frac{\pi_{x}^{2}}{2 \alpha_{1}^{2} \pi}+\frac{\pi_{y}^{2}}{2 \alpha_{1}^{2} \pi}\right) \lambda_{\sigma}=\pi \tilde{w}_{0}
$$

en $\sigma=1$ y coincide con (6.61) pero, siguiendo a [38] imponemos $\tilde{w}_{0}=0$ [eq. (6.59)], simplificando y definiendo $\alpha^{2} \equiv \alpha_{1}^{2} / \alpha_{2}^{2}$, para obtener las CFN reportadas en [27,p787]

$$
\lambda_{\sigma}=\pi \frac{\pi_{x} \lambda_{x}+\pi_{y} \lambda_{y}}{\alpha^{2}+\pi_{x}^{2}+\pi_{y}^{2}} \text { en } \sigma=1
$$

Esta condición es inconsistente. Si, por ejemplo, consideramos un campo $\mathbf{v}^{0}=u \mathbf{i}, v_{0}=w_{0}=0$, usado en $[38$, eq 12], las componentes contravariantes de $\mathbf{v}^{0}$ son $\tilde{u}_{0}=u_{0}, \tilde{v}_{0}=0, \quad \tilde{w}_{0}=-\sigma \frac{u_{0}}{\pi} \pi_{x}$ y la CFN natural (6.61) toma la forma

$$
\lambda_{\sigma}=\pi \frac{2 u_{0} \pi_{x}+\pi_{x} \lambda_{x}+\pi_{y} \lambda_{y}}{\alpha^{2}+\pi_{x}^{2}+\pi_{y}^{2}} \quad \text { en } \sigma=1
$$

en lugar de (6.63). El problema en [38] reside en considerar $\delta \tilde{u}, \delta \tilde{v}, \delta \tilde{w}$ como las componentes de $\mathbf{v}-\mathbf{v}^{0}$. Estas variaciones son las componentes contravariantes de un elemento arbitrario de $\mathbb{V}_{y}$. Tenemos $\nabla \cdot\left(\mathbf{v}-\mathbf{v}^{0}\right)=$ $-\nabla \cdot \mathbf{v}^{0} \neq 0$ y por lo tanto $\mathbf{v}-\mathbf{v}^{0} \notin \mathbb{V}_{y}$. Si consideramos $\delta \tilde{w}=\tilde{w}-\tilde{w}_{0}$ en $\sigma=1$ y un $\tilde{w}_{0}$ no necesariamente cero obtenemos la condición incorrecta (6.63). Los autores de [38] encontraron que, para flujos simples, $\alpha$ varía entre 0.1 a 1 . Una comparación entre (6.63) y (6.64) muestra que la CFN natural (6.64) tiene un término adicional $2 u_{0} \pi_{x} \pi /\left(\alpha^{2}+\pi_{x}^{2}+\pi_{y}^{2}\right)$ el cual puede ser grande incluso para $\alpha^{2} \in\left[10^{-2}, 1\right]$. Por ejemplo, en algunos casos reportados en [38] los autores consideran $u_{0} \sim 1 \mathrm{~ms}^{-1}, \pi=500 \mathrm{~m}$.

Bernard et. al. [2] proponen una técnica de optimización para estimar automáticamente y de forma precisa los parámetros $\alpha$ en un MMC junto con las CFN

$$
\frac{\partial \lambda}{\partial \mathbf{n}}=\mathbf{n} \cdot \nabla \lambda=0
$$

Los autores definen $\tau=\log _{e} \alpha^{2}$ y encuentran en sus simulaciones que $\tau=-0,25\left(\alpha^{2}=0,8\right)$ da por resultado un campo ajustado cercano a los datos. Podemos justificar, en parte, dicho resultado por la cercanía entre las CFN (6.65) y las condiciones de frontera (6.28) para $\alpha^{2} \sim 1,0$ [2]. De hecho, los autores usan las coordenadas (6.57) y explícitamente señalan [2, pág 677] que sobre superficies sólidas la derivada normal $\partial \lambda / \partial n$ debe ser cero para que no se ajuste la componente normal, y esta condición de frontera, junto con la condición de que la componentes del flujo inicial sean paralelas a la superficie, satisfacen la condición de impenetrabilidad (ver también [22, 251]. En consecuencia, la componente contravariante $v^{0,3}(y)$ de el campo inicial deben ser cero. En este caso la CFN natural (6.28) es

$$
\frac{z_{x}}{z_{\sigma} \alpha_{1}^{2}} \lambda_{x}+\frac{z_{y}}{z_{\sigma} \alpha_{2}^{2}} \lambda_{y}-\mathbb{M}_{33}^{-1} \lambda_{\sigma}=0
$$


y la forma de (6.65) viene de (6.66) con $\alpha_{i}=1$. La diferencia entre (6.65) y (6.66) es significativa cuando $\alpha$ varía en el intervalo $\left[10^{-12}, \infty\right)$ considerado en la literatura [22]. A continuación se presentan unos ejemplos numéricos

Consideremos la región $\overline{\mathcal{D}}=[0,1] \times[h(x), 1+h(x)]$ con $h=2-2 x+x^{2} / 2$. Las coordenadas son $y^{1}=x$, $y^{3}=\sigma=z-h(x)$, de ahí $z_{x}=-2+x, z_{\sigma}=1, \overline{\mathcal{D}}_{y}=[0,1] \times[0,1]$. El campo $\mathbf{v}^{0}$ es $u^{0}=v_{0}[z-h(x)], w^{0}=0$ con $v_{0}=10 \mathrm{~ms}^{-1}$ y su componente contravariante es

$$
v^{0,1}(y)=v_{0} \sigma, \quad v^{0,3}(y)=-z_{x} u^{0}
$$

donde $V^{0,3}(y)=0$ en $\sigma=0(6.52)$. El problema elíptico (6.26) para $\lambda$ es

$$
-\frac{\lambda_{x x}}{\alpha_{1}^{2}}+2 \frac{z_{x} \lambda_{x \sigma}}{\alpha_{1}^{2}}+\frac{\lambda_{\sigma}}{\alpha_{1}^{2}}-\mathbb{M}_{33}^{-1} \lambda_{\sigma \sigma}=u_{x}^{0}-z_{x} u_{\sigma}^{0}
$$

con $\mathbb{M}_{33}^{-1}=z_{x}^{2} / \alpha_{1}^{2}+1 / \alpha_{3}^{2}(6.32,6.53)$. La CFN (6.54) es

$$
\frac{z_{x}}{\alpha_{1}^{2}} \lambda_{x}-\left(\frac{z_{x}^{2}}{\alpha_{1}^{2}}+\frac{1}{\alpha_{3}^{2}}\right) \lambda_{\sigma}=0 \text { en } \sigma=0
$$

y la CFN (6.65) viene de (6.68) con $\alpha_{i}=1$,

$$
z_{x} \lambda_{x}-\left(z_{x}^{2}+1\right) \lambda_{\sigma}=0
$$

Los cálculos fueron hechos con $\alpha_{1}=1$ y la variable $\alpha_{3}$. Sean $\mathbf{v}_{n}\left(\alpha_{3}^{-2}\right)$ y $\mathbf{v}_{a}\left(\alpha_{3}^{-2}\right)$ los campos ajustados obtenidos de las CFN naturales (6.68) y de las CFN "artificiales"(6.69), respectivamente. Estos campos son estimados resolviendo (6.67) con el mismo esquema de diferencias finitas. Se usa una malla uniforme $\left\{x_{i}, \sigma_{j}\right\}$ para discretizar (6.68) con un esquema de diferencias de cinco puntos [11]. Sean $\lambda_{i j} \equiv \lambda\left(x_{i}, \sigma_{j}\right)$, las ecuaciones $(6.68,6.69)$ son discretizadas con

$$
\begin{aligned}
\frac{\partial \lambda\left(x_{i}, \sigma_{j}\right)}{\partial x} & \sim \frac{\lambda_{i-1, j}-\lambda_{i+1, j}}{2 h} \\
\frac{\partial \lambda\left(x_{i}, \sigma_{j}\right)}{\partial \sigma} & \sim \frac{\lambda_{i, j+1}-\lambda_{i, j}}{h} .
\end{aligned}
$$

Los campos $\mathbf{v}_{n}$ y $\mathbf{v}_{a}$ coinciden con $\alpha_{3}^{2}=1$, la gráfica correspondiente en el espacio $x z$ se muestra en la figura (6.1). Un resultado similar se obtiene con $\alpha_{3}^{2}=10^{+4}$ cuyos campos coinciden y la gráfica es mostrada en la figura (6.2). Sin embargo, para $\alpha_{3}^{-2}=10^{+4} \operatorname{los}$ campos $\mathbf{v}_{n}\left(10^{+4}\right)$ y $\mathbf{v}_{a}\left(10^{+4}\right)$ son bastante diferentes como se muestra en la figura (6.3). Según la estimación del cociente $\alpha=\alpha_{1} / \alpha_{3}$ por medio de el cociente esperado $w / u$, hemos usado $\alpha_{3}^{-2} \in\left[10^{-4}, 10^{0}\right]$. En este rango $\mathbf{v}_{n}\left(\alpha_{3}^{-2}\right)$ y $\mathbf{v}_{a}\left(\alpha_{3}^{-2}\right)$ son prácticamente el mismo campo. Esto puede explicar por qué la inconsistencia de las CFN (6.65) no ha sido reportada en la literatura. Sin embargo, los ejemplos de [31]-[4] sugieren que $\alpha=\alpha_{1} / \alpha_{3}$ puede escogerse en el rango $\left[10^{+4}, \infty\right)$ donde los campos $\mathbf{v}_{n}$ y $\mathbf{v}_{a}$ son bastante diferentes. Creemos que el número de datos utilizado por autores anteriores no era suficiente para estimar el campo verdadero, lo cual permitiría poner en evidencia la inconsistencia de las CFN (6.65). Algunos autores [38] han intentado poner a prueba la confiabilidad de los MMC usando campos ideales analíticos $\mathbf{v}$, pero estos estudios son parciales ya que $\lambda$ fue calculada con los parámetros $\alpha_{i}=1$ los cuales no consideran los casos de interés $\alpha_{1} / \alpha_{3} \ll 1$ y $\alpha_{1} / \alpha_{3} \gg 1$. 


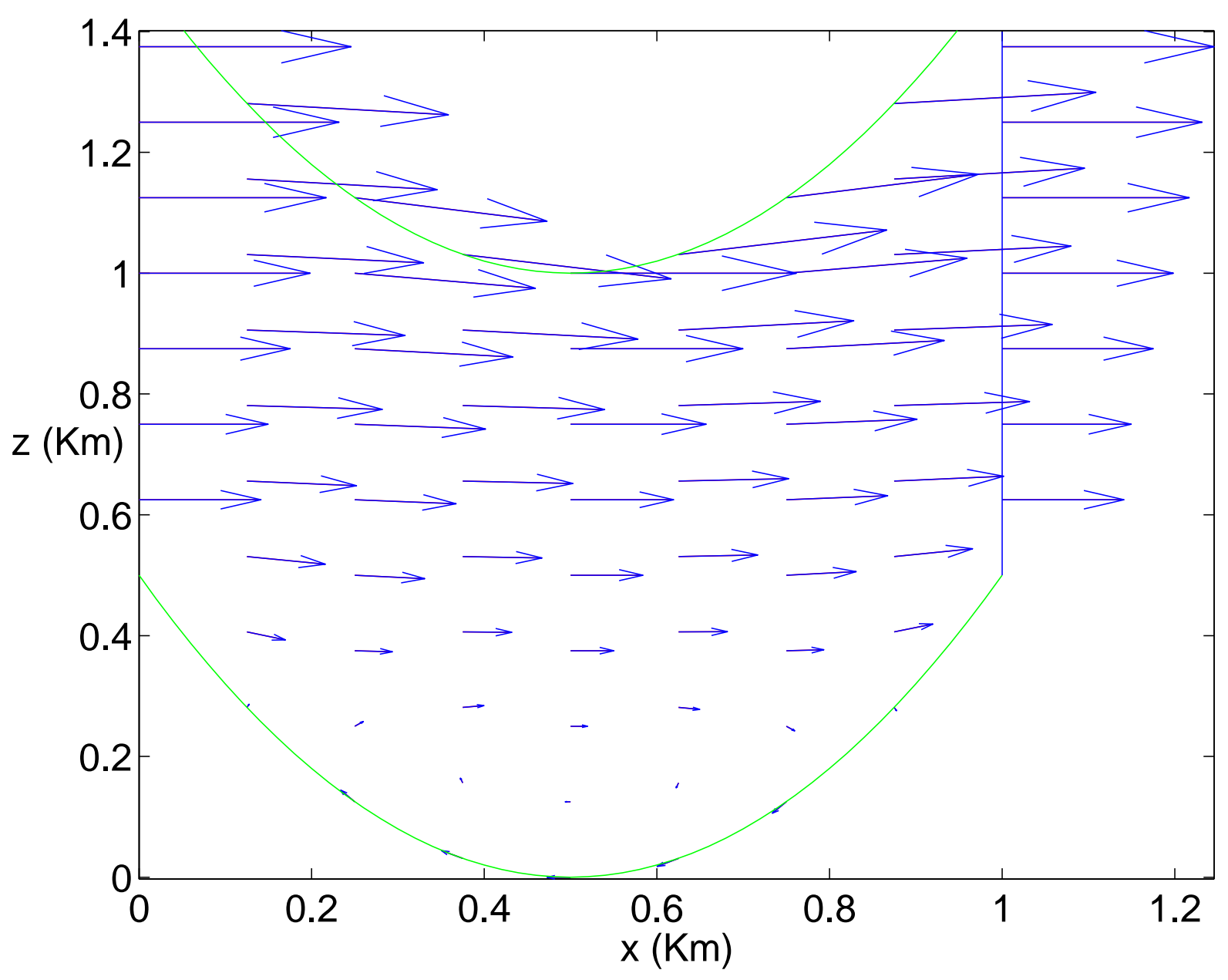

Figura 6.1: Campos $\mathbf{v}_{n}(\rightarrow)$ y $\mathbf{v}_{a}(-)$ con $\left(\alpha^{2}=1\right)$. 


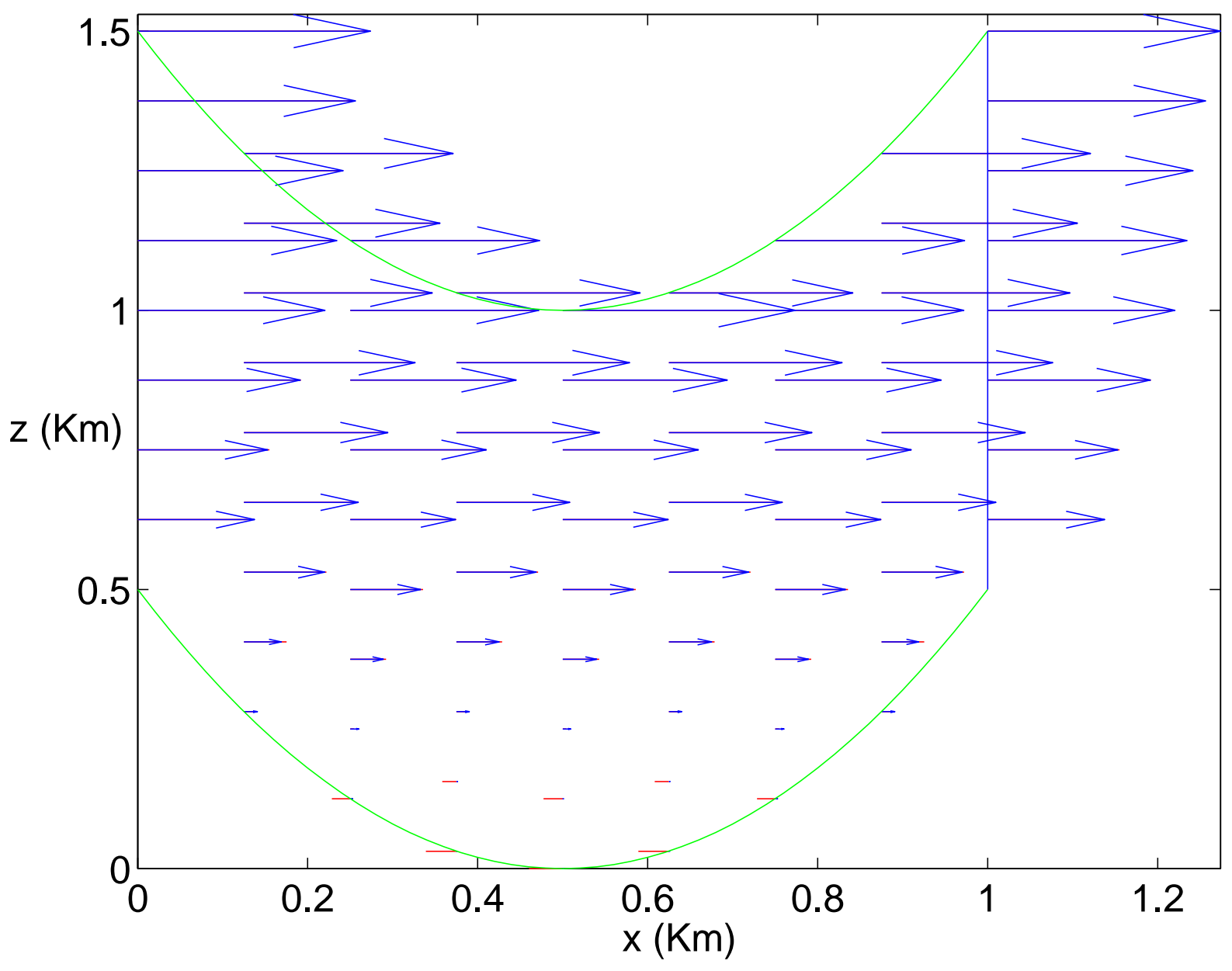

Figura 6.2: Campos $\mathbf{v}_{n}(\rightarrow)$ y $\mathbf{v}_{a}(-)$ con $\left(\alpha^{2}=10^{-4}\right)$. 


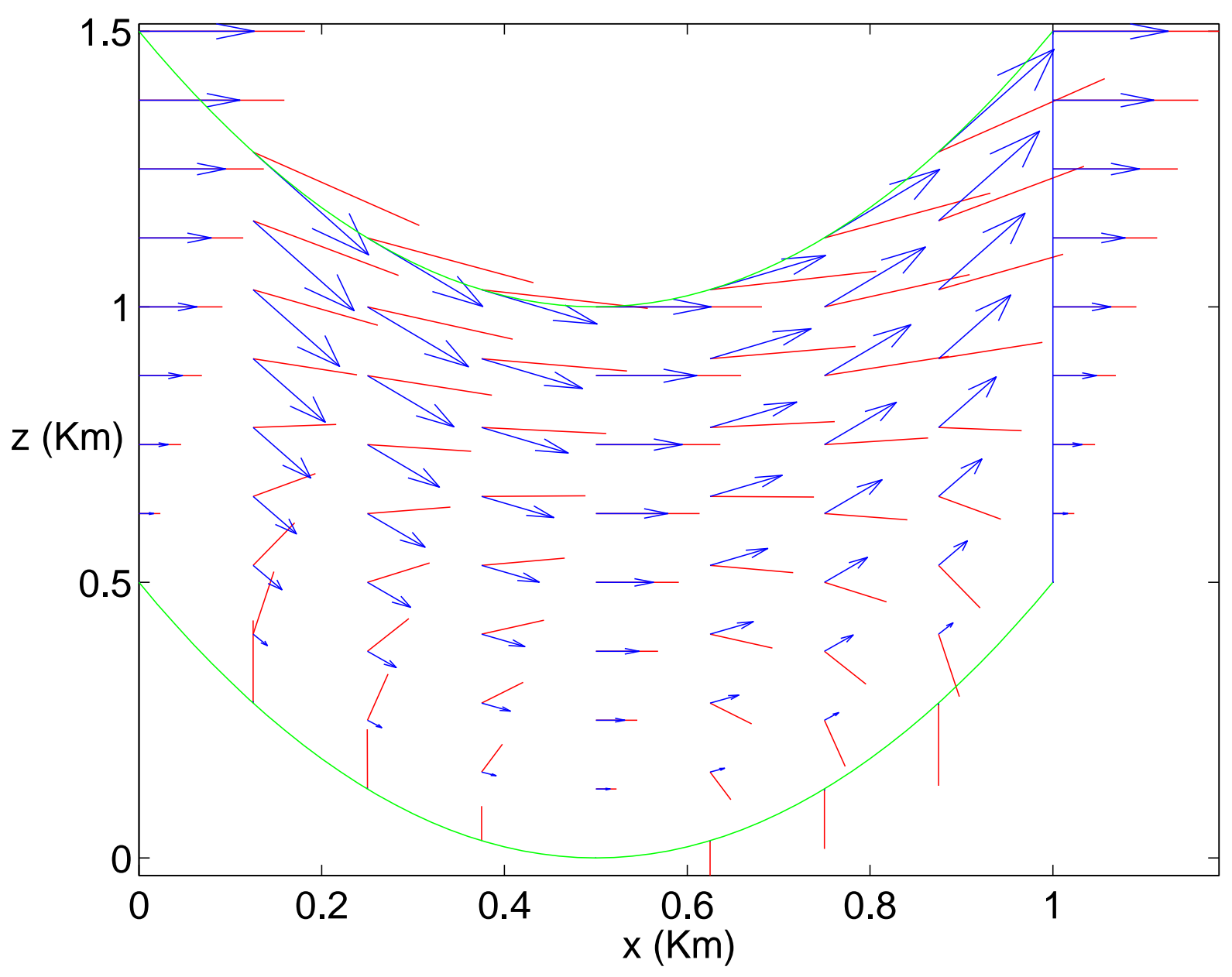

Figura 6.3: Campos $\mathbf{v}_{n}(\rightarrow)$ y $\mathbf{v}_{a}(-)$ con $\left(\alpha^{2}=10^{+4}\right)$. 


\section{Capítulo 7}

\section{Cálculo de $\mathbf{v}(\mathbf{r})$ con la restricción $\nabla \cdot \rho_{0}(\mathbf{r}) \mathbf{v}=0$}

Para analizar la importancia de la estratificación vertical en la sección 6.1 planteamos el cálculo de un campo ajustado $\mathbf{v}(\mathbf{r})$ con la restricción $\nabla \cdot \rho_{0} \mathbf{v}=0$. El cálculo en coordenadas generalizadas como referencia de futuros trabajos. En la sección 7.1 consideramos un ejemplo sencillo en el cual el cálculo de $\lambda$ se reduce a la solución de una familia de problemas de Sturm-Liouville. Los resultados numéricos reportados en la sección 7.6 muestran que la variación de $\rho_{0}(\mathbf{r})$ puede ignorarse a lo más en una región de análisis con una altura de $2 \mathrm{~km}$. En un trabajo reciente [37] se han reportado cálculos del campo ajustado en una región con $5 \mathrm{~km}$ de altura considerando a $\rho_{0}$ constante mientras que nuestros resultados demuestran que el error en $\mathbf{v}(\mathbf{r})$ aumenta rápidamente con relación al cálculo de $\mathbf{v}(\mathbf{r})$ con $\rho_{0}(\mathbf{r})$ variable, hasta un $20 \%$.

\subsection{Cálculo en coordenadas generalizadas}

En esta sección consideramos el cálculo del campo ajustado en coordenadas generalizadas usando como restricción dinámica la ecuación de continuidad profunda

$$
\nabla \cdot \rho_{0} \mathbf{v}=0
$$

donde $\rho_{0}$ es variable y la condición de resbalamiento

$$
\mathbf{w} \cdot \mathbf{n}=0 \quad \text { sobre } \quad \Gamma_{N y} .
$$

con $\mathbf{n}$ un vector normal unitario exterior a $\Gamma$ en el espacio físico $x^{1} x^{2} x^{3}$. La forma generalizada de estas restricciones es

$$
\begin{aligned}
\mathbf{w} \cdot \mathbf{n} & =w^{i} \tau_{i} \cdot n_{j} \eta^{j}=w^{i}(y) n_{i}(y) \\
\nabla \cdot \rho_{0} \mathbf{w} & =\frac{1}{\sqrt{g}} \frac{\partial}{\partial y^{i}} \sqrt{g} \rho_{0} w^{i}(y)
\end{aligned}
$$

donde $n_{j}=$ componente covariante de $\mathbf{n}$. El espacio al que pertenece $\mathbf{v}$ es

$$
\mathbb{N}_{y} \equiv\left\{\mathbf{w}: \nabla \cdot \rho_{0} \mathbf{w}=0 \text { en } \mathcal{D}_{y},\left.\mathbf{n} \cdot \mathbf{w}\right|_{\Gamma_{N}}=0\right\}
$$

y el funcional a minimizar es

$$
J_{y}(\mathbf{w})=\left\|\mathbf{w}-\mathbf{v}^{0}\right\|_{\mathcal{D} M}^{2}
$$

donde $\|\mathbf{u}\|_{\mathcal{D} M}$ es la norma asociada al producto interior en el espacio $\mathbf{L}^{2}\left(\mathcal{D}_{y}\right)=\left\{W^{i=1,2,3}(y) \in L_{g}^{2}\left(\mathcal{D}_{y}\right)\right\}$

$$
\langle\mathbf{w} \mid \mathbf{u}\rangle_{D M}=\int_{\mathcal{D} y}\left(\mathbf{w}^{\eta}\right)^{t} \mathbb{M} \mathbf{u}^{\eta} \sqrt{g} d y
$$


$\mathbb{M} \equiv \mathbb{J}^{t} \mathbb{S} \mathbb{J}$, y $L_{g}^{2}\left(\mathcal{D}_{y}\right)$ tiene el producto interior $\langle f, q\rangle_{g}=\int_{\mathcal{D}_{y}} f q \sqrt{g} d y$.

Si $\mathbf{v}$ es una extremal de $J_{y}(\mathbf{w})$ en $\mathbb{N}_{y}$ debe satisfacer

$$
\left.\frac{d J_{y}(\mathbf{v}+\epsilon \mathbf{w})}{d \epsilon}\right|_{\epsilon=0}=0 \quad \forall \mathbf{w} \in \mathbb{N}_{y}
$$

donde

$$
J_{y}(\mathbf{v}+\epsilon \mathbf{w})=\|\Delta \mathbf{v}+\epsilon \mathbf{w}\|_{\mathcal{D} M}^{2}=\|\Delta \mathbf{v}\|_{\mathcal{D} M}^{2}+2 \epsilon\langle\Delta \mathbf{v} \mid \mathbf{w}\rangle_{\mathcal{D} M}+\epsilon^{2}\|\mathbf{w}\|_{\mathcal{D} M}^{2}
$$

por lo tanto

$$
\int_{\mathcal{D} y} \mathbf{w}^{T} \mathbb{M} \Delta \mathbf{v} \sqrt{g} d y=0 \quad \forall \mathbf{w} \in \mathbb{N}_{y} .
$$

Para introducir la restricción $\nabla \cdot \rho_{0} \mathbf{w}=0$ consideramos $\lambda \nabla \cdot \rho_{0} \mathbf{w}=0$ en la forma $\nabla \cdot \lambda \rho_{0} \mathbf{w}-\nabla \lambda \cdot \rho_{0} \mathbf{w}=0$ e integramos

$$
\int_{\mathcal{D} y}\left[\nabla \cdot \lambda \rho_{0} \mathbf{w}-\nabla \lambda \cdot \rho_{0} \mathbf{w}\right] \sqrt{g} d y=0
$$

Usando a w y $\nabla \lambda$ en su forma contravariante y covariante, respectivamente,

$$
\mathbf{w}=\mathbf{w}^{i}(y) \tau_{i} \quad \nabla \lambda=\eta^{j} \frac{\partial \lambda}{\partial y^{i}},
$$

obtenemos

$$
\nabla \cdot \lambda \rho_{0} \mathbf{w}=\frac{1}{\sqrt{g}} \frac{\partial}{\partial y^{i}} \sqrt{g} \lambda \rho_{0} w^{i} \quad \nabla \lambda \cdot \rho_{0} \mathbf{w}=\rho_{0} \frac{\partial \lambda}{\partial y^{i}} \sqrt{g} w^{i} .
$$

Aplicando el teorema de la divergencia en el espacio $y$ obtenemos

$$
\int_{\mathcal{D} y}\left[\nabla \cdot \lambda \rho_{0} \mathbf{w}\right] \sqrt{g} d y=\int_{\mathcal{D} y} \frac{1}{\sqrt{g}}\left(\frac{\partial}{\partial y^{i}} \sqrt{g} \lambda \rho_{0} w^{i}\right) \sqrt{g} d y=\int_{\mathcal{D} y} \frac{\partial}{\partial y^{i}} \sqrt{g} \lambda \rho_{0} w^{i} d y=\oint_{\Gamma y} \sqrt{g} \lambda \rho_{0} w^{i} \hat{n}_{i} d s_{y}
$$

donde $\hat{n}_{i}=$ componente del vector normal unitario exterior a $\Gamma_{y}$. De acuerdo con la relación $n_{i}=\sqrt{g} \hat{n}_{i}$ entre las componentes covariantes $n_{i}$ del vector normal unitario exterior $\mathbf{n}$ a $\Gamma$ y $\hat{n}_{i}$ tenemos $w^{i} \sqrt{g} \hat{n}_{i}=\mathbf{w} \cdot \mathbf{n}$ y reescribimos (7.7)

$$
\int_{\mathcal{D} y}\left[\nabla \cdot \lambda \rho_{0} \mathbf{w}\right] \sqrt{g} d y=\oint_{\Gamma y} \lambda \rho_{0} \mathbf{w} \cdot \mathbf{n} d s_{y} .
$$

Por otro lado

$$
\int_{\mathcal{D} y}\left[\nabla \lambda \cdot \rho_{0} \mathbf{w}\right] \sqrt{g} d y=\left\langle\mathbf{w} \mid \rho_{0} \nabla \lambda\right\rangle_{D y} .
$$

En esta forma $(7.6,7.5)$ queda como sigue:

$$
\oint_{\Gamma_{y}} \lambda \rho_{0} \mathbf{w} \cdot \mathbf{n} d s_{y}-\left\langle\mathbf{w} \mid \rho_{0} \nabla \lambda\right\rangle_{\mathcal{D} y}=0 .
$$

Pero $\Gamma_{y}=\left.\Gamma_{D y} \cup \Gamma_{N y} \mathrm{y} \mathbf{w} \cdot \mathbf{n}\right|_{\Gamma_{D y}}=0$ lo que nos lleva a

$$
\int_{\Gamma_{N y}} \lambda \rho_{0} \mathbf{w} \cdot \mathbf{n} d s_{y}-\left\langle\mathbf{w} \mid \rho_{0} \nabla \lambda\right\rangle_{\mathcal{D} y}=0 .
$$

Sumando (7.5) con (7.8)

$$
\left\langle\mathbf{w} \mid \mathbb{M} \Delta \mathbf{v}-\rho_{0} \nabla \lambda\right\rangle_{\mathcal{D} y}+\int_{\Gamma_{N y}} \lambda \rho_{0} \mathbf{w} \cdot \mathbf{n} d s_{y}=0 \quad \forall \mathbf{w} \in \mathbb{N}_{y} .
$$

En particular, si $\mathbf{w}\left(\Gamma_{y}\right)=0$, queda

$$
\left\langle\mathbf{w} \mid \mathbb{M} \Delta \mathbf{v}-\rho_{0} \nabla \lambda\right\rangle_{\mathcal{D} y}=0
$$


de donde se obtiene la ecuación de Euler-Lagrange

$$
\mathbf{v}(y)=\mathbf{v}^{i}(y)+\rho_{0} \mathbb{M}^{-1} \nabla \lambda \quad \text { ó } \quad v^{i}(y)=v^{0 i}(y)+\rho_{0} \mathbb{M}_{i j}^{-1} \frac{\partial \lambda(y)}{\partial y^{i}} .
$$

Dado que (7.10) no depende de $\mathbf{w}$, es válida para toda $\mathbf{w}$ en $\mathbb{N}_{y}$ con lo cual (7.9) se reduce a

$$
\int_{\Gamma_{N y}} \lambda \rho_{0} \mathbf{w} \cdot \mathbf{n} d s_{y}=0 \quad \forall \mathbf{w} \in \mathbb{V}_{y}
$$

lo que es posible sí y sólo si $\lambda$ satisface la condición de frontera Dirichlet

$$
\lambda=0 \quad \text { sobre } \quad \Gamma_{D y} .
$$

Para calcular $\lambda$ sustituimos (7.10) en la forma generalizada de $\nabla \cdot \rho_{0} \mathbf{v}=0$ y haciendo algunos cálculos

$$
\begin{aligned}
\frac{1}{\sqrt{g}} \frac{\partial}{\partial y^{i}} \sqrt{g} \rho_{0} v^{i} & =0 \\
\frac{1}{\sqrt{g}} \frac{\partial}{\partial y^{i}} \sqrt{g} \rho_{0}\left[v^{0 i}+\rho_{0} \mathbb{M}_{i j}^{-1} \frac{\partial \lambda}{\partial y^{j}}\right] & =0 \\
-\frac{1}{\sqrt{g}} \frac{\partial}{\partial y^{i}} \sqrt{g} \rho_{0}^{2} \mathbb{M}_{i j}^{-1} \frac{\partial \lambda}{\partial y^{j}}-\frac{\partial}{\partial y^{i}} \sqrt{g} \rho_{0} v^{0 i} & =0
\end{aligned}
$$

se llega a la ecuación elíptica

$$
L_{y} \lambda=\sqrt{g} \nabla \cdot \rho_{0} v^{0}
$$

donde

$$
\begin{aligned}
L_{y} & \equiv-\frac{1}{\sqrt{g}} \frac{\partial}{\partial y^{i}} \sqrt{g} \rho_{0}^{2} \mathbb{M}_{i j}^{-1} \frac{\partial}{\partial y^{j}}=-\nabla_{y}^{T} \sqrt{g} \rho_{0}^{2} \mathbb{M}^{-1} \nabla_{y} . \\
\sqrt{g} \nabla \cdot \rho_{0} \mathbf{v}^{0} & =\frac{\partial}{\partial y^{i}} \sqrt{g} \rho_{0} \mathbf{v}^{0 i}
\end{aligned}
$$

La condición $\mathbf{v} \cdot \mathbf{n}=0$ sobre $\Gamma_{N y}$ toma la forma $\mathbf{n} \cdot \mathbf{v}=\mathbf{n}^{t}\left[\mathbf{v}^{0}+\rho_{0} \mathbb{M}^{-1} \nabla \lambda\right]=0$. Sustituyendo $\mathbf{n}=\sqrt{g} \hat{\mathbf{n}} \mathrm{y}$ multiplicando por $\rho_{0}$ obtenemos

$$
\mathcal{L}_{y} \lambda=-\rho_{0} \hat{\mathbf{n}} \cdot \mathbf{v}^{0}
$$

donde aparece la derivada conormal

$$
\mathcal{L}_{y} \equiv \hat{\mathbf{n}}^{t} \rho_{0}^{2} \mathbb{M}^{-1} \nabla
$$

asociada al operador $L_{y}$ y en términos de la cual la condición $\mathbf{v} \cdot \mathbf{n}=0$ sobre $\Gamma_{N y}$ constituye una condición de frontera natural para el problema elíptico (7.12).

\subsubsection{Forma débil del problema elíptico asociado a $\lambda$}

El multiplicador $\lambda$ pertenece al espacio $L_{2}\left(\mathcal{D}_{y}\right)=\left\{v: \int_{\mathcal{D}_{y}}|v|^{2} d y<\infty\right\}$ con el producto interior

$$
\langle f \mid g\rangle_{y} \equiv \int_{\mathcal{D} y} f(y) g(y) d y
$$

donde no aparece el jacobiano $\sqrt{g}$, así como al espacio

$$
H_{D y}^{1}=\left\{\phi \in H^{1}\left(\mathcal{D}_{y}\right): \phi=0 \text { sobre } \Gamma_{y}\right\}
$$

Para obtener la forma débil del problema elíptico (7.12), tenemos la identidad

$$
\left\langle\phi \mid L_{y} \lambda\right\rangle=-\oint_{\Gamma_{y}} \phi \mathcal{L}_{y} \lambda \sqrt{g} d s_{y}+a(\phi, \lambda) \quad \text { válida para } \phi, \lambda \in H_{D y}^{1}
$$


que se obtiene integrando por partes

$$
\begin{aligned}
\left\langle\phi \mid L_{y} \lambda\right\rangle & =\int_{\mathcal{D} y} \phi\left(-\frac{\partial}{\partial y^{i}} \sqrt{g} \rho_{0}^{2} \mathbb{M}_{i j}^{-1} \frac{\partial \lambda}{\partial y^{j}}\right) d y \\
& =-\int_{\mathcal{D} y} \phi \frac{\partial}{\partial y^{i}}\left(\sqrt{g} \rho_{0}^{2} \mathbb{M}_{i j}^{-1} \frac{\partial \lambda}{\partial y^{j}}\right) d y \\
& =-\left[\oint_{\Gamma_{y}} \phi \sqrt{g} \rho_{0}^{2} \mathbb{M}_{i j}^{-1} \frac{\partial \lambda}{\partial y^{j}} \hat{n}_{i} d s_{y}-\int_{\mathcal{D} y} \rho_{0}^{2} \frac{\partial \phi}{\partial y^{i}} \mathbb{M}_{i j}^{-1} \frac{\partial \lambda}{\partial y^{j}} \sqrt{g} d y\right] \\
& =-\int_{\Gamma_{D y}} \phi \mathcal{L}_{y} \lambda d s_{y}+a(\phi, \lambda),
\end{aligned}
$$

donde usamos: (i) $\phi=0$ sobre $\Gamma_{D y}$, (ii) la definición de $\mathcal{L}_{y}$, y (iii) definimos

$$
a(\phi, \lambda)=\int_{\Gamma_{N y}} \rho_{0}^{2} \frac{\partial \phi}{\partial y^{i}} \mathbb{M}_{i j}^{-1} \frac{\partial \lambda}{\partial y^{j}} \sqrt{g} d y=\int_{D y} \nabla^{T} \phi \rho_{0}^{2} \mathbb{M}^{-1} \nabla \lambda \sqrt{g} d y .
$$

Así, la ecuación (7.12) toma la forma

$$
a(\phi, \lambda)=\int_{\Gamma_{N y}} \phi \mathcal{L}_{y} \lambda \sqrt{g} d s_{y}+\left\langle\phi \mid \sqrt{g} \nabla \cdot \rho_{0} V^{0}\right\rangle_{y}
$$

Imponiendo la condición $\mathcal{L}_{y} \lambda=-\rho_{0} \mathbf{v}^{0} \cdot \hat{\mathbf{n}}$ obtenemos la forma débil del problema ( 7.12 ), a saber,

$$
a(\phi, \lambda)=\left\langle\phi \mid \sqrt{g} \nabla \cdot \rho_{0} \mathbf{v}^{0}\right\rangle_{y}-\int_{\Gamma_{N}} \phi \rho_{0} \mathbf{v}^{0} \cdot \hat{\mathbf{n}} \sqrt{g} d s_{y} \quad \forall \phi \in H_{\mathcal{D}}^{1}\left(D_{y}\right) .
$$

El lado derecho de (7.15) puede simplificarse con la identidad $\nabla \cdot \phi \rho_{0} \mathbf{v}^{0}=\nabla \phi \cdot \rho_{0} \mathbf{v}^{0}+\phi \nabla \cdot \rho_{0} \mathbf{v}^{0}$ la cual equivale a

$$
\sqrt{g}\left[\phi \nabla \cdot \rho_{0} \mathbf{v}^{0}\right]=\sqrt{g}\left[\nabla \cdot \phi \rho_{0} \mathbf{v}^{0}-\nabla \phi \cdot \rho_{0} \mathbf{v}^{0}\right]=\sqrt{g}\left[\frac{1}{\sqrt{g}} \frac{\partial}{\partial y^{i}} \sqrt{g} \phi \rho_{0} v^{0, i}-\nabla \phi \cdot \rho_{0} \mathbf{v}^{0}\right] .
$$

Integrando

$$
\left\langle\phi \mid \sqrt{g} \nabla \cdot \rho_{0} \mathbf{v}^{0}\right\rangle_{y}=\int_{\mathcal{D} y} \frac{\partial}{\partial y^{i}} \sqrt{g} \phi \rho_{0} v^{0, i} d y-\int_{\mathcal{D} y} \nabla \phi \cdot \rho_{0} \mathbf{v}^{0} \sqrt{g} d y
$$

y usando la identidad de Green junto con $\phi=0$ sobre $\Gamma_{D y}$,

$$
\int_{\mathcal{D} y} \frac{\partial}{\partial y^{i}} \sqrt{g} \phi \rho_{0} v^{0, i} d y=\oint_{\Gamma_{y}} \phi \sqrt{g} \rho_{0} v^{0, i} \hat{n}_{i} d s_{y}=\int_{\Gamma_{N}} \phi \rho_{0} \mathbf{v}^{0} \cdot \hat{\mathbf{n}} \sqrt{g} d s_{y},
$$

se obtiene

$$
\left\langle\phi \mid \sqrt{g} \nabla \cdot \rho_{0} v^{0}\right\rangle_{y}=\int_{\Gamma_{N y}} \phi \rho_{0} \mathbf{v}^{0} \cdot \hat{\mathbf{n}} \sqrt{g} d s_{y}-\int_{\mathcal{D} y} \nabla \phi \cdot \rho_{0} \mathbf{v}^{0} \sqrt{g} d y
$$

y reemplazando en el lado derecho de ( 7.15 ) llegamos a la identidad

$$
\left\langle\phi \mid \sqrt{g} \nabla \cdot \rho_{0} \mathbf{v}^{0}\right\rangle_{y}-\int_{\Gamma_{N}} \phi \rho_{0} \mathbf{v}^{0} \cdot \hat{\mathbf{n}} \sqrt{g} d s_{y}=\int_{\mathcal{D} y} \nabla \phi \cdot \rho_{0} \mathbf{v}^{0} \sqrt{g} d y \quad \forall \phi \in H_{D}^{1}\left(\mathcal{D}_{y}\right) .
$$

Sustituyendo lo anterior en (7.15) obtenemos

$$
a(\phi, \lambda)=-\left\langle\nabla \phi \mid \rho_{0} v^{0}\right\rangle_{\mathcal{D} y} \quad \forall \phi \in H_{D}^{1}\left(\mathcal{D}_{y}\right)
$$

con

$$
\left\langle\nabla \phi \mid \rho_{0} v^{0}\right\rangle_{\mathcal{D} y}=\int_{\mathcal{D} y} \nabla \phi \cdot \rho_{0} \mathbf{v}^{0} \sqrt{g} d y=\int_{\mathcal{D} y} \rho_{0} v^{0, i} \frac{\partial \phi}{\partial y^{i}} \sqrt{g} d y
$$

que es una forma adecuada para el cálculo de $\lambda$ con el método de elemento finito. 


\subsubsection{Problema variacional asociado a $\lambda$}

Para obtener la formulación variacional del problema débil ( 7.16 ) definamos

$$
f_{y}(\phi) \equiv\left\langle\phi \mid \sqrt{g} \nabla \cdot \rho_{0} \mathbf{v}^{0}\right\rangle_{y}-\oint_{\Gamma_{n y}} \phi \sqrt{g} \rho_{0} v^{0} \cdot \hat{n} d s_{y}=-\left\langle\nabla \phi \mid \rho_{0} v^{0}\right\rangle_{D y}
$$

con lo cual (7.17) toma la forma

$$
a(\phi, \lambda)=f_{y}(\phi) \quad \forall \phi \in H_{D}^{1}\left(\mathcal{D}_{y}\right)
$$

Las extremales del funcional

$$
J(\phi)=\frac{1}{2} a(\phi, \phi)-f_{y}(\phi) \quad \operatorname{con} \phi \in H_{D}^{1}\left(\mathcal{D}_{y}\right)
$$

satisfacen (7.18). En efecto la condición de extremo es

$$
\left.\frac{d}{d \epsilon} J(\lambda+\epsilon \phi)\right|_{\epsilon=0}=0
$$

con

$$
J(\lambda+\epsilon \phi)=\frac{1}{2}\left[a(\lambda, \lambda)+\epsilon a(\lambda, \phi)+\epsilon^{2} a(\phi, \phi)\right]-f(\phi)-\epsilon f(\phi)
$$

equivale a $a(\lambda, \phi)=f_{y}(\phi)$ que es exactamente (7.18).

\subsection{Flujo alrededor de un cilindro circular}

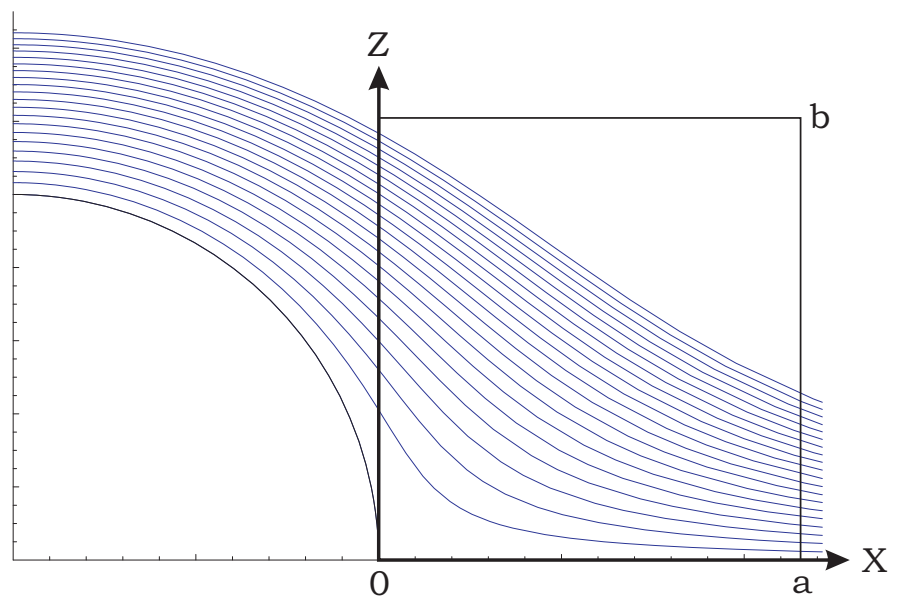

Figura 7.1: Líneas de corriente del flujo alrededor de un cilindro

El campo de velocidad del flujo alrededor de un cilindro $\mathbf{v}_{c}=u_{c} \hat{\mathbf{x}}+w_{c} \hat{\mathbf{z}}$ es

$$
u_{c}(x, z)=u_{0}+u_{0} \frac{r_{c}^{2}}{r^{4}}\left(Z^{2}-X^{2}\right) \quad w(x, z)=-u_{0} \frac{r_{c}^{2}}{r^{4}} X Z
$$

donde

$$
Z=z \quad X=x+r_{c} \quad r^{2}=X^{2}+Z^{2} .
$$

El campo $\mathbf{v}_{c}$ satisface

$$
\nabla \cdot \mathbf{v}_{c}=0 \quad \text { con }\left.\quad \mathbf{v}_{c} \cdot \hat{\mathbf{n}}\right|_{z=0}=\left.\mathbf{v}_{c} \cdot \hat{\mathbf{z}}\right|_{z=0}=0
$$


pero esto es irrelevante ya que el propósito inmediato es estudiar la importancia de imponer la restricción

$$
\nabla \cdot \rho_{0}(z) \mathbf{v}=0
$$

en lugar de $\nabla \cdot \mathbf{v}=0$ para calcular un campo ajustado $\bar{V}_{a}$ a partir de un campo inicial que, en principio, es arbitrario $\mathbf{v}^{0}$. Un primer campo inicial $\mathbf{v}^{0} \equiv u^{0} \hat{\mathbf{x}}+w^{0} \hat{\mathbf{z}}$ es

$$
\begin{aligned}
& u^{0}(x, z) \equiv u(x, z)=u_{0}+u_{0} \frac{r_{c}^{2}}{r^{4}}\left(Z^{2}-X^{2}\right) \\
& w^{0}(x, z) \equiv 0 .
\end{aligned}
$$

el cual satisface la condición de resbalamiento

$$
\left.\mathbf{v} \cdot \hat{\mathbf{n}}\right|_{z=h(x)}=\left.\mathbf{v} \cdot \hat{\mathbf{z}}\right|_{z=0}=\left.\mathbf{w}^{0}\right|_{z=0}=0 .
$$

Sin embargo, para simplificar los cálculos numéricos consideraremos un campo inicial $u^{0}(x, z)$ definido por interpolación lineal del flujo alrededor de un cilindro. Sea

$$
u_{i}(z)=u_{0}\left(R_{i}, z\right)=u_{0}+u \frac{r_{c}^{2}}{r^{4}}\left(Z^{2}-X^{2}\right) \quad \text { con } \quad X=x+\left.r_{0}\right|_{x=R_{i}}
$$

el valor de campo "real" a lo largo de dos radiosondeos lanzados en $R_{1}$ y $R_{2}$, entonces definimos

$$
u^{0}(x, z)=\frac{x-R_{2}}{R_{1}-R_{2}} u_{1}(z)+\frac{x-R_{1}}{R_{2}-R_{1}} u_{2}(z)
$$

Este campo coincide con los radiosondeos,

$$
\left.u^{0}(x, z)\right|_{x=R_{i}}=u_{i}=u\left(R_{i}, z\right) .
$$

Otra expresión de $u^{0}(x, z)$ es

de la cual obtenemos

$$
u^{0}(x, z)=\frac{R_{2} u_{1}-R_{1} u_{2}}{R_{2}-R_{1}}+\left(\frac{u_{2}-u_{1}}{R_{2}-R_{1}}\right) x
$$

$$
\frac{\partial u^{0}}{\partial x}=\frac{u_{2}(z)-u_{1}(z)}{R_{2}-R_{1}} .
$$

El campo ajustado $\mathbf{v}$ es aquel que minimiza al funcional $F(\mathbf{w})=\left\|\mathbf{w}-V^{0}\right\|_{\Omega s}^{2}$ y satisface

$$
\nabla \cdot \rho_{0}(z) \mathbf{v}=0 \quad \text { en } \quad \mathcal{D}_{y}
$$

con

El campo v está dado por

$$
\mathbf{v} \cdot \hat{n}=0 \quad \text { en } \quad z=h(x) .
$$

donde $\lambda$ es la solución de problema

$$
\mathbf{v}=v^{0}+\rho_{0} S^{-1} \nabla \lambda
$$

$$
\begin{array}{rlrlrl}
L \lambda & =\nabla \cdot \rho_{0} v^{0} & & \\
\lambda & =0 & & \text { sobre } & \Gamma_{D} \\
\mathcal{L} \lambda & =-\rho_{0} v^{0} \cdot \hat{n} & \text { sobre } & \Gamma_{N}=\{z=h(x)=0\} .
\end{array}
$$

La ecuación elíptica para $\lambda$ puede reescribirse como

$$
\left(\alpha_{1}^{2} L_{x}+\alpha_{3}^{-2} L_{z}\right) \lambda=F(x, z)
$$

donde definimos

$$
L_{x} \equiv-\partial_{x}^{2}, \quad L_{z} \equiv-\frac{1}{\rho_{0}^{2}} \frac{\partial}{\partial z} \rho_{0}^{2} \frac{\partial}{\partial z}, \quad F(x, z) \equiv \frac{\nabla \cdot \rho_{0}^{2} v^{0}}{\rho_{0}^{2}},
$$

$\mathrm{y}$ las condiciones de frontera sobre $\lambda$ toman la forma

$$
\begin{aligned}
\lambda & =0 & \text { en } & & x=0, x_{\text {máx }} ; z=z_{\text {máx }} \\
\frac{\partial \lambda}{\partial z} & =0 & \text { en } & z & =0 .
\end{aligned}
$$




\subsection{Problema de Sturm-Liouville asociado a $\lambda$}

Para calcular $\lambda$ consideramos la forma

$$
\lambda=\sum_{n=1}^{\infty} c_{n}(z) u_{n}(x), \quad F=\sum_{n=1}^{\infty} F_{n}(z) u_{n}(x)
$$

donde las funciones

$$
u_{n}(x)=\sqrt{\frac{2}{a}} \sin \omega_{n} x \quad \text { con } \quad \omega_{n}=\frac{n \pi}{a}, \quad a \equiv x_{\text {máx }},
$$

forman un base completa de $L^{2}\left(0, x_{\text {máx }}\right)$ que satisface las condiciones de frontera Dirichlet en $x=0, x_{\text {máx }}, \mathrm{y}$

$$
F_{n}(z)=\int_{0}^{a} u_{n}(x) F(x, z) d x .
$$

Calculemos en forma más detallada la última integral. Tenemos

$$
F_{n}(z)=\int_{0}^{a} \sqrt{\frac{2}{a}} \sin \omega_{n} x \frac{\nabla \cdot \rho_{0} \mathbf{v}^{0}}{\rho_{0}^{2}(z)} d x=\sqrt{\frac{2}{a}} \frac{1}{\rho_{0}^{2}(z)} \int_{0}^{a} \sin \omega_{n} x \nabla \cdot \rho_{0} \mathbf{v}^{0} d x
$$

Para un campo inicial de la forma $\mathbf{v}^{0} \equiv u^{0} \hat{\imath}$

$$
\nabla \cdot \rho_{0}(z) \mathbf{v}^{0}=\frac{\partial}{\partial x} \rho_{0}(z) u^{0}(x, z)=\rho_{0}(z) \frac{\partial}{\partial x} u^{0}(x, z),
$$

por lo tanto

$$
F_{n}(z)=\sqrt{\frac{2}{a}} \frac{1}{\rho_{0}(z)} \int_{0}^{a} \sin \omega_{n} x \frac{\partial}{\partial x} u^{0}(x, z) d x
$$

En el caso particular del campo $\mathbf{v}^{0} \equiv u^{0} \hat{\imath}$ definido por interpolación lineal de dos radiosondeos, $\frac{\partial u^{0}}{\partial x}$ no depende de $x$ lo que nos permite evaluar inmediatamente la integral anterior

$$
\int_{0}^{a} \sin \omega_{n} x \frac{\partial u^{0}}{\partial x} d x=\frac{\partial u^{0}}{\partial x}\left[-\frac{\cos \omega_{n} x}{\omega_{n}}\right]_{0}^{a}=\frac{\partial u^{0}}{\partial x} \frac{1-\cos \omega_{n} a}{\omega_{n}}
$$

pero $\omega_{n} a=n \pi, \cos \omega_{n} a=\cos n \pi=(-1)^{n}$, por lo tanto

$$
F_{n}(z)=\sqrt{\frac{2}{a}} \frac{1}{\rho_{0}(z)} \frac{\partial u^{0}}{\partial x} \frac{1-(-1)^{n}}{\omega_{n}}=\left\{\begin{array}{lll}
\frac{2}{\omega_{n}} \sqrt{\frac{2}{a}} \frac{1}{\rho_{0}(z)} \frac{\partial u^{0}}{\partial x} & \text { para } & n=1,3,5, \ldots \\
0 & \text { para } & n=2,4,6, \ldots
\end{array} .\right.
$$

En esta forma sólo debemos resolver

$$
\left(\alpha_{3}^{-2} L_{z}+E_{n} \alpha_{1}^{-2}\right) c_{n}(z)=F_{n}(z) \quad \text { para } \quad n=1,3,5, \ldots
$$

con

$$
\frac{d c_{n}(0)}{d z}=c(b)=0, \quad E_{n} \equiv \omega_{n}^{2}, \quad F_{n}(z)=\frac{2}{\omega_{n}} \sqrt{\frac{2}{a}} \frac{1}{\rho_{0}(z)} \frac{\partial u^{0}}{\partial x} .
$$

Reescribiendo (7.19),

$$
\left[\alpha_{3}^{-2}\left(-\frac{1}{\rho_{0}^{2}} \frac{d}{d z} \rho_{0}^{2} \frac{d}{d z}\right)+E_{n} \alpha_{1}^{-2}\right] c_{n}(z)=F_{n}(z)
$$

obtenemos

$$
\frac{1}{\rho_{0}^{2}}\left[-\frac{d}{d z} \rho_{0}^{2} \frac{d}{d z}+E_{n} \frac{\alpha_{3}^{2}}{\alpha_{1}^{2}} \rho_{0}^{2}(z)\right] c_{n}(z)=\alpha_{3}^{2} F_{n}(z)
$$

que es una caso particular del problema de Sturm-Liouville

$$
\frac{1}{r(z)}\left[-\partial_{z} p(z) \partial_{z}+q(z)\right] u=f
$$


con

$$
r(z)=\rho_{0}^{2}(z) \quad p(z)=\rho_{0}^{2}(z) \quad q(z)=E_{n} \frac{\alpha_{3}^{2}}{\alpha_{1}^{2}} \rho_{0}^{2}(z) \quad f(z)=\alpha_{3}^{2} F_{n}(z) .
$$

En términos del operador

$$
A \equiv \frac{1}{r}\left[-\partial_{z} p \partial_{z}+q\right]
$$

podemos escribir

$$
A u=f .
$$

Para obtener la formulación variacional de este problema consideremos $\varphi \in C_{0}^{\infty}(0, b)$, entonces

$$
\begin{aligned}
\varphi A u & =f \varphi \\
(\varphi A u) r & =f \varphi r \\
\int_{0}^{b}(\varphi A u) r d z & =\int_{0}^{b} f \varphi r d z .
\end{aligned}
$$

donde aparece el producto interior

$$
\langle f \mid g\rangle_{r} \equiv \int_{0}^{b} f g r d z
$$

Integrando por partes

$$
\begin{aligned}
\langle\varphi \mid A u\rangle_{r} & =\int_{0}^{b}(\varphi A u) r d z=\int_{0}^{b} \varphi\left[-\partial_{z} p \partial_{z}+q\right] u d z \\
& =-\int_{0}^{b} \varphi \partial_{z} p \partial_{z} u d z+\int_{0}^{b} \varphi q u d z \\
& =-\left[\varphi p \partial_{z} u\right]_{0}^{b}+\int_{0}^{b} p \dot{\varphi} \dot{u} d z+\int_{0}^{b} \varphi q u d z \\
& =a(u, \varphi),
\end{aligned}
$$

donde definimos

$$
a(u, \varphi) \equiv\langle p \dot{\varphi} \mid \dot{u}\rangle+\langle q \varphi \mid u\rangle
$$

se concluye que $u$ debe satisfacer

$$
a(u, \varphi) \equiv\langle f \mid g\rangle_{r} \quad \text { para cada } \varphi \in C_{0}^{\infty}(0, b) .
$$

Esto nos lleva a buscar a $u(z)$ como la extremal del funcional

$$
J(v) \equiv \frac{1}{2} a(v, v)-F(v), \quad \text { con } F(v) \equiv\langle f \mid v\rangle_{r},
$$

sobre el espacio $\mathbb{V}_{b}=\left\{v \in H^{1}(0, b): v(b)=0\right\}$. El operador $A$ es simétrico en $L_{2}(0, b, r)$ con el dominio

$$
\mathcal{D}(A)=\left\{v \in H^{1}(0, b): \dot{v}(0)=v(b)=0\right\} .
$$

y el teorema de Lax-Milgram garantiza que existe una única función $u$ en $\mathbb{V}_{b}$ que minimiza a $J(v)$.

\subsection{Solución aproximada del problema de Sturm-Liouville con el método del elemento finito}

Sea $\left\{\phi_{j}(x)\right\}_{j=1}^{N}$ un conjunto linealmente independiente del espacio $\mathbb{V}_{b}$. Proponemos

$$
u_{N}=\sum_{j=1}^{N} u_{j} \phi_{j}(x)
$$


y sustituimos en $J\left(u_{N}\right)$,

$$
J\left(u_{N}\right)=\frac{1}{2} \sum_{j k} a\left(\phi_{k}, \phi_{j}\right) u_{k} u_{j}-\sum_{k} F\left(\phi_{k}\right) u_{k}
$$

Minimizando

$$
\frac{\partial J}{\partial u_{i}}=\frac{1}{2} \sum_{j k} a\left(\phi_{k}, \phi_{j}\right)\left(\delta_{k i} u_{j}+u_{k} \delta i j\right)-\sum_{k} F\left(\phi_{k}\right) \delta_{i k}=0
$$

y usando la simetría de $a(\cdot, \cdot)$

se llega al sistema de ecuaciones algebraicas

$$
\frac{1}{2}\left[\sum_{j} a_{i j} u_{j}+\sum_{k} a_{k i} u_{k}\right]-F\left(\phi_{i}\right)=0
$$

$$
\sum_{j} a_{i j} u_{j}=F_{i}
$$

donde $a_{i j} \equiv a\left(\phi_{k}, \phi_{j}\right)$ y $F_{i} \equiv F\left(\phi_{i}\right)$. Sea $\left\{x_{i}=(i-1) h\right\}_{i=1}^{n-1}$ una malla uniforme sobre el intervalo [0,b], con $x_{1}=0$ y $x_{n}=b$. El conjunto $\left\{\phi_{j}(x)\right\}_{j=1}^{N}$ que consideraremos son las funciones base gorro

$$
\begin{aligned}
& \phi_{1}(x)= \begin{cases}\frac{x_{2}-x}{h} & x_{1} \leq x \leq x_{2} \\
0 & x \geq x_{2}\end{cases} \\
& \phi_{i}(x)=\left\{\begin{array}{rl}
0 & x \leq x_{i-1} \\
\frac{x-x_{i-1}}{h} & x_{i-1} \leq x \leq x_{i} \\
\frac{x_{i+1}-x}{h} & x_{i} \leq x \leq x_{i+1} \\
0 & x \geq x_{i+1}
\end{array} \quad \text { para } i=2, \ldots, n-1\right.
\end{aligned}
$$

las cuales tienen la propiedad $\phi_{j}\left(x_{k}\right)=\delta_{j k}$, es decir,

$$
\phi_{j}\left(x_{j}\right)=1 \quad \phi_{i}\left(x_{j}\right)=0,
$$

lo que aprovechamos para calcular los elementos de matriz $a_{k j}$ y $F_{i}$ con la regla del trapecio.

1. Dado que la matriz $a_{i j}$ es simétrica, solo calcularemos la parte triangular superior. Para $a_{11}$ tenemos

$$
a_{11}=\left\langle\dot{\phi}_{1} \mid \dot{\phi}_{1}\right\rangle_{p}+\left\langle q \phi_{1} \mid \phi_{1}\right\rangle_{p}
$$

donde

$$
\begin{aligned}
\dot{\phi}_{1} & =-\frac{1}{h} \\
\left\langle p \dot{\phi}_{1} \mid \dot{\phi}_{1}\right\rangle & =\frac{1}{h^{2}} \int_{0}^{h} p(x) d x=\frac{1}{h^{2}} \frac{h}{2}[p(0)+p(h)]=\frac{1}{2 h}[p(0)+p(h)] \\
\left\langle q \phi_{1} \mid \phi_{1}\right\rangle & =\int_{0}^{h} q \phi_{1}^{2}(x) d x=\frac{h}{2}\left[\left(q \phi_{1}^{2}\right)_{z=0}+\left(q \phi_{1}^{2}\right)_{z=h}\right]=\frac{h}{2} q(0) .
\end{aligned}
$$

En forma análoga

$$
\begin{aligned}
a_{12} & =\left\langle p \dot{\phi}_{1} \mid \dot{\phi}_{2}\right\rangle+\left\langle q \phi_{1} \mid \phi_{2}\right\rangle \\
& =\int_{0}^{h}\left(-\frac{1}{h}\right)\left(\frac{1}{h}\right) p d x+\int_{0}^{h} q \phi_{1} \phi_{2} d x=-\frac{1}{h^{2}} \int_{0}^{h} p d x+\int_{0}^{h} q \phi_{1} \phi_{2} p d x \\
& =\left(-\frac{1}{h^{2}}\right) \frac{h}{2}[p(0)+p(h)]+\frac{h}{2}[0] \\
& =-\frac{1}{2 h}[p(0)+p(h)]
\end{aligned}
$$

los elementos $a_{1 j}$ con $j \geq 3$ son cero. 
2. Para $2 \leq i \leq n$ solo los elementos $a_{i i}$ y $a_{i, i+1}$ son diferentes de 0 :

$$
\begin{aligned}
a_{i i} & =\left\langle p \dot{\phi}_{i} \mid \dot{\phi}_{i}\right\rangle+\left\langle q \phi_{i} \mid \phi_{i}\right\rangle=\int_{x_{i-1}}^{x_{i+1}} \dot{\phi}^{2} p d x+\int_{x_{i-1}}^{x_{i+1}} q \phi_{i}^{2} d x \\
& =\int_{x_{i-1}}^{x_{i}} \frac{1}{h^{2}} p d x+\int_{x_{i}}^{x_{i+1}} \frac{1}{h^{2}} p d x+\int_{x_{i-1}}^{x_{i}} q \phi_{i}^{2} d x+\int_{x_{i}}^{x_{i+1}} q \phi_{i}^{2} d x \\
& =\frac{1}{h^{2}}\left[p_{i}+p_{i-1}+p_{i}+p_{i+1}\right] \frac{h}{2}+\frac{h}{2}\left[\left(q \phi_{i}^{2}\right)_{x_{i-1}}+\left(q \phi_{i}^{2}\right)_{x_{i}}\right]+\frac{h}{2}\left[\left(q \phi_{i}^{2}\right)_{x_{i}}+\left(q \phi_{i}^{2}\right)_{x_{i+1}}\right] \\
& =\frac{1}{2 h}\left[p_{i-1}+2 p_{i}-p_{i+1}\right]+h q_{i}
\end{aligned}
$$

donde usamos la notación $p\left(x_{i}\right) \equiv p_{i}, q\left(x_{i}\right) \equiv q_{i}, \mathrm{y}$

$$
\begin{aligned}
a_{i, i+1} & =\left\langle\dot{\phi}_{i} \mid \dot{\phi}_{i+1}\right\rangle+\left\langle q \phi_{i} \mid \phi_{i+1}\right\rangle=\int_{x_{i}}^{x_{i+1}}\left(-\frac{1}{h^{2}}\right) p d x+\int_{x_{i}}^{x_{i+1}} q \phi_{i} \phi_{i+1} p d x \\
& =-\frac{1}{2 h}\left[p_{i}+p_{i+1}\right]
\end{aligned}
$$

3. Para el vector $F_{i}=F\left(\phi_{i}\right)$ tenemos

$$
\begin{aligned}
F_{i} & =\left\langle f \mid \phi_{i}\right\rangle_{r}=\int_{x_{i-1}}^{x_{i+1}} f \phi_{i} r d x=\int_{x_{i-1}}^{x_{i}} f \phi_{i} r d x+\int_{x_{i}}^{x_{i+1}} f \phi_{i} r d x \\
& =\frac{h}{2}(f r)_{x_{i}}+\frac{h}{2}(f r)_{x_{i}}=h f_{i} r_{i}
\end{aligned}
$$

\subsection{Cálculo del campo ajustado con elemento finito}

De acuerdo con el método del elemento finito la solución aproximada del problema ( 7.19 ) tiene la forma

$$
c_{N n}(z)=\sum_{j=1}^{N} c_{N n j} \phi_{j}(z)
$$

y procedemos calcular las correcciones al campo ajustado

$$
\begin{aligned}
& \Delta V^{1} \equiv u_{a}-u_{a}^{0}=\frac{\rho_{0}(z)}{\alpha_{1}^{2}} \sum_{n=1,3,5, \ldots} c_{N n}(z) \frac{d u_{n}(x)}{d x} \\
& \Delta V^{1} \equiv w_{a}-w_{a}^{0}=\frac{\rho_{0}(z)}{\alpha_{3}^{2}} \sum_{n=1,3,5, \ldots} \frac{d c_{N n}(z)}{d z} u_{n}(x) .
\end{aligned}
$$

El cálculo de la derivada en $\Delta V^{1}$ es inmediato. El cálculo de $\frac{d}{d z} c_{N n}(z)$ lo hacemos en forma débil como sigue. Proponemos

$$
\frac{d c_{N n}}{d z}=\sum_{j=1}^{N} \xi_{N n j} \phi_{j}(z)
$$

donde $\xi_{N n j}$ es un conjunto de coeficientes indeterminados, multiplicando por $\phi_{i}(z)$ e integrando

$$
\int_{0}^{b} \phi_{i} \frac{d c_{N n}}{d z} d z=\sum_{j=1}^{N} \xi_{N n j} \int_{0}^{b} \phi_{i} \phi_{j} d z \quad 1 \leq i \leq N .
$$

Si definimos

$$
M_{i j}=\int_{0}^{b} \phi_{i} \phi_{j} d z, \quad g_{i}=\int_{0}^{b} \phi_{i} \frac{d c_{n}^{(N)}}{d z} d z
$$


y $\xi_{N n}$ es el vector columna con componentes $\xi_{N n j}$, observamos que (7.20) define el sistema de ecuaciones

$$
\mathbb{M} \xi_{N n}=g
$$

para los coeficientes $\xi_{N n j}$. Usemos la regla del trapecio y la relación de ortogonalidad $\phi_{i}\left(z_{j}\right)=\delta_{i j}$ para calcular $M_{i j}$. Para $i=1$ tenemos

$$
\begin{aligned}
& M_{11}=\int_{0}^{b} \phi_{1}^{2}(z) d z=\int_{z_{1}}^{z_{2}} \phi_{1}^{2}(z) d z=\frac{h}{2}\left[\phi_{1}^{2}\left(z_{1}\right)+\phi_{1}^{2}\left(z_{2}\right)\right]=\frac{h}{2} \\
& M_{12}=\int_{0}^{b} \phi_{1} \phi_{2} d z=\int_{0}^{h} \phi_{1} \phi_{2} d z=\frac{h}{2}\left[\phi_{1}(0) \phi_{2}(0)+\phi_{1}(h) \phi_{2}(h)\right]=0,
\end{aligned}
$$

y para $2 \leq i \leq N$

$$
\begin{aligned}
M_{i i} & =\int_{0}^{b} \phi_{i} \phi_{i} d z=\int_{z_{i-1}}^{z_{i}} \phi_{i}^{2} d z+\int_{z_{i}}^{z_{i+1}} \phi_{i}^{2} d z=\frac{h}{2}\left[\left(\phi_{i}^{2}\right)_{z_{i-1}}^{z_{i}}+\left(\phi_{i}^{2}\right)_{z_{i}}^{z_{i+1}}\right] \\
& =\frac{h}{2}\left[\phi_{i}^{2}\left(z_{i-1}\right)+2 \phi_{i}^{2}\left(z_{i}\right)+\phi_{i}^{2}\left(z_{i+1}\right)\right]=h \\
M_{i, i+1} & =\int_{0}^{b} \phi_{i} \phi_{i+1} d z=\frac{h}{2}\left[\left(\phi_{i} \phi_{i+1}\right)_{z_{i}}+\left(\phi_{i} \phi_{i+1}\right)_{z_{i+1}}\right]=0 .
\end{aligned}
$$

Entonces

$$
\mathbb{M}=\left[\begin{array}{cccc}
h / 2 & 0 & \ldots & 0 \\
0 & h & & 0 \\
\vdots & & \ddots & \vdots \\
0 & 0 & \ldots & h
\end{array}\right]
$$

por lo que los coeficientes $\xi_{N n j}$ están dados por

$$
\begin{aligned}
\xi_{1} & =\frac{2}{h} g_{1} \\
\xi_{j} & =\frac{1}{h} g_{j} \quad \text { para } j=2, \ldots, N .
\end{aligned}
$$

Integrando directamente

$$
\begin{aligned}
g_{1} & =\int_{0}^{b} \phi_{1} \frac{d c_{n}}{d z} d z=\int_{z_{1}}^{z_{2}} \phi_{1} \sum_{j=1}^{N} c_{j} \dot{\phi}_{j} d z=\sum_{j=1}^{N} c_{j} \int_{z_{1}}^{z_{2}} \phi_{1} \dot{\phi}_{j} d z=\sum_{j=1,2} c_{j} \int_{z_{1}}^{z_{2}} \phi_{1} \dot{\phi}_{j} d z \\
& =\left\{c_{1} \frac{h}{2}\left[\phi_{1} \dot{\phi}_{1}\left|z_{1}+\phi_{1} \dot{\phi}_{1}\right| z_{2}\right]+c_{2} \frac{h}{2}\left[\phi_{1} \dot{\phi}_{2}\left|z_{1}+\phi_{1} \dot{\phi}_{2}\right| z_{2}\right]\right\}=\frac{h}{2}\left\{-\frac{1}{h} c_{1}+\frac{1}{h} c_{2}\right\},
\end{aligned}
$$

obtenemos

y para $g_{j \geq 2}$

$$
\xi_{1}=\frac{c_{2}-c_{1}}{h}
$$

$$
\begin{aligned}
g_{j \geq 2} & =\int_{0}^{b} \phi_{j} \frac{d c_{n}}{d z} d z=\int_{z_{j-1}}^{z_{j+1}} \phi_{j} \sum_{k=1}^{N} c_{k} \dot{\phi}_{k}=\sum_{k=j-1, j, j+1} c_{k} \int_{z_{j-1}}^{z_{j+1}} \phi_{j} \dot{\phi}_{k} d z \\
& =\sum_{k=j-1, j, j+1} c_{k}\left\{\int_{z_{j-1}}^{z_{j}} \phi_{j} \dot{\phi}_{k} d z+\int_{z_{j}}^{z_{j+1}} \phi_{j} \dot{\phi}_{k} d z\right\} \\
& =c_{j-1} \int_{z_{j-1}}^{z_{j}} \phi_{j}\left(\frac{-1}{h}\right)+c_{j} \int_{z_{j-1}}^{z_{j}} \phi_{j}\left(\frac{1}{h}\right) d z+c_{j} \int_{z_{j}}^{z_{j+1}} \phi_{j}\left(\frac{-1}{h}\right) d z+c_{j+1} \int_{z_{j}}^{z_{j+1}} \phi_{j}\left(\frac{1}{h}\right) d z \\
& =\frac{1}{h}\left\{-c_{j-1} \int_{z_{j-1}}^{z_{j}} \phi_{j} d z+c_{j} \int_{z_{j-1}}^{z_{j}} \phi_{j} d z-c_{j} \int_{z_{j}}^{z_{j+1}} \phi_{j} d z+c_{j+1} \int_{z_{j}} d z\right\} \\
& =\frac{1}{h} \frac{h}{2}\left\{-c_{j-1}+c_{j}-c_{j}+c_{j+1}\right\}
\end{aligned}
$$


tenemos

$$
\xi_{j \geq 2}=\frac{c_{j+1}-c_{j-1}}{2 h}
$$

\subsection{Resultados numéricos}

En la figura (7.2) podemos ver la gran diferencia que hay entre la densidad constante y las densidades calculadas en (Estados de referencia hidrostáticos). En las figuras (7.3 - 7.4) podemos apreciar la comparación entre el campo ajustado con densidad constante y los campos ajustados considerando la densidad variable. En estas gráficas no es claro que exista una diferencia significativa, sin embargo en (7.5) se muestran los errores relativos de la componente vertical del campo ajustado con densidad constante con respecto a los campos ajustados con densidad variable y en éstas se puede apreciar que el error relativo puede llegar a ser mayor al $10 \%$ en tan sólo $2 \mathrm{~km}$ de altura. Es por esto que señalamos la importancia de resolver $\nabla \cdot \rho_{0} \mathbf{v}=0$

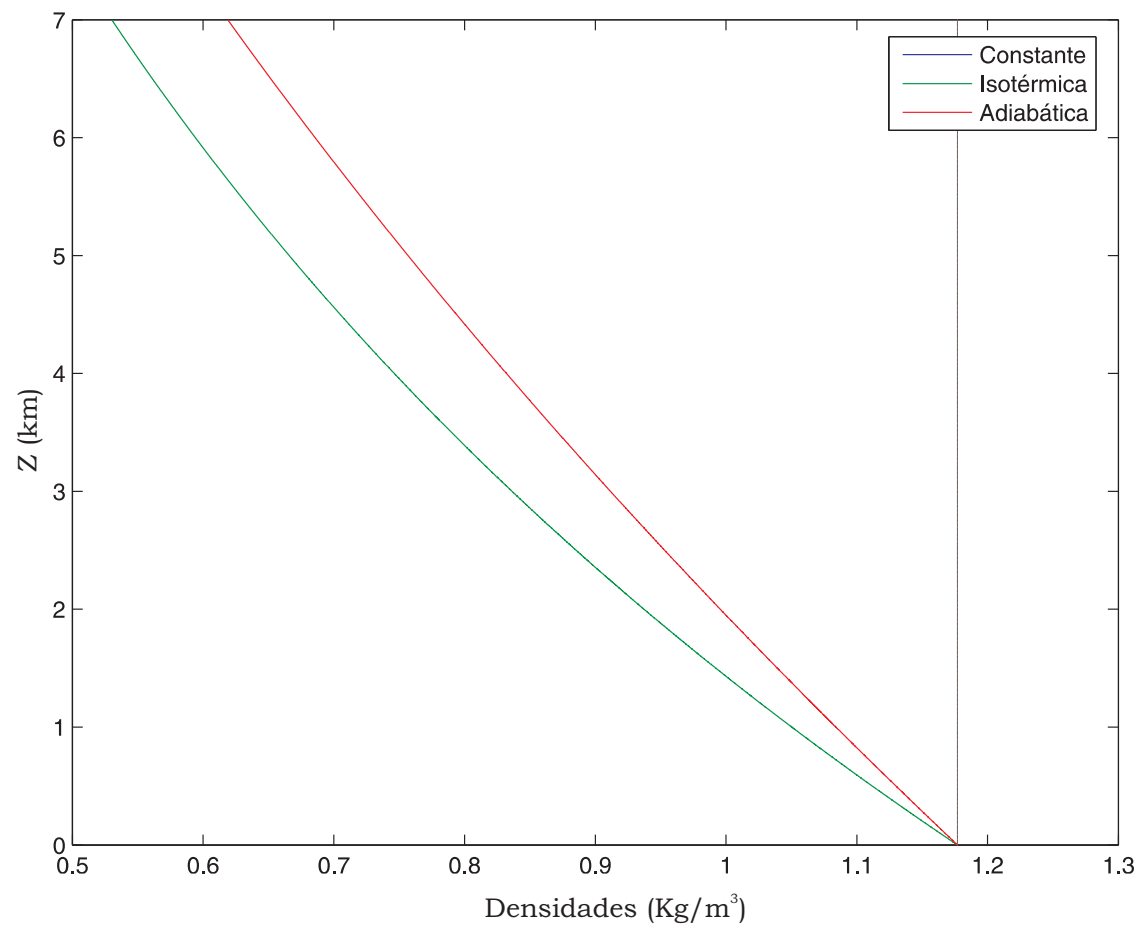

Figura 7.2: Gráficas de las densidades utilizadas para ajustar el campo de viento 


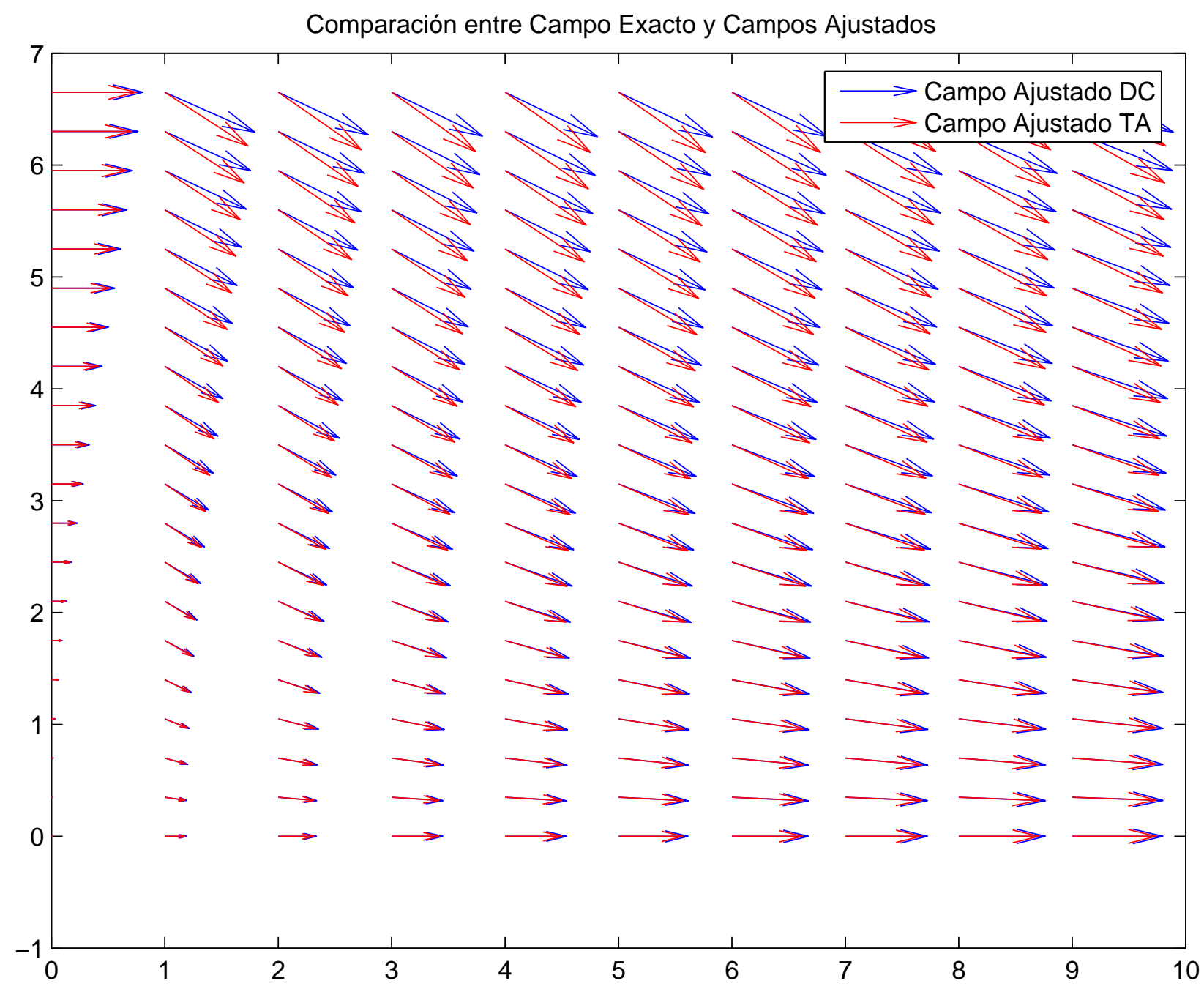

Figura 7.3: Comparación entre los campos de viento ajustados con densidad constante y variable. $($ TA $=$ Termodinámica Adiabática) 


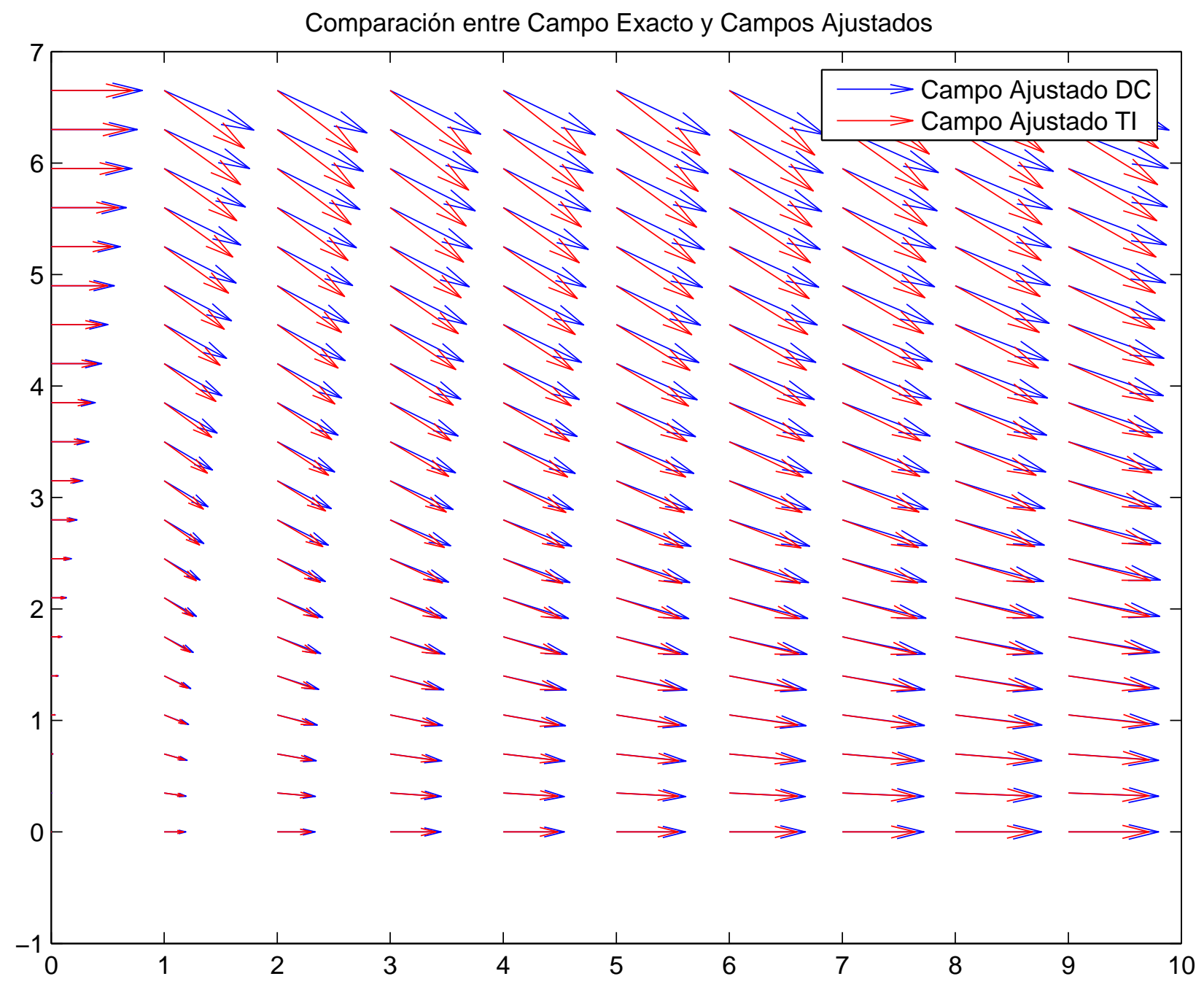

Figura 7.4: Comparación entre los campos de viento ajustados con densidad constante y variable $($ TI $=$ Termodinámica Isotérmica) 

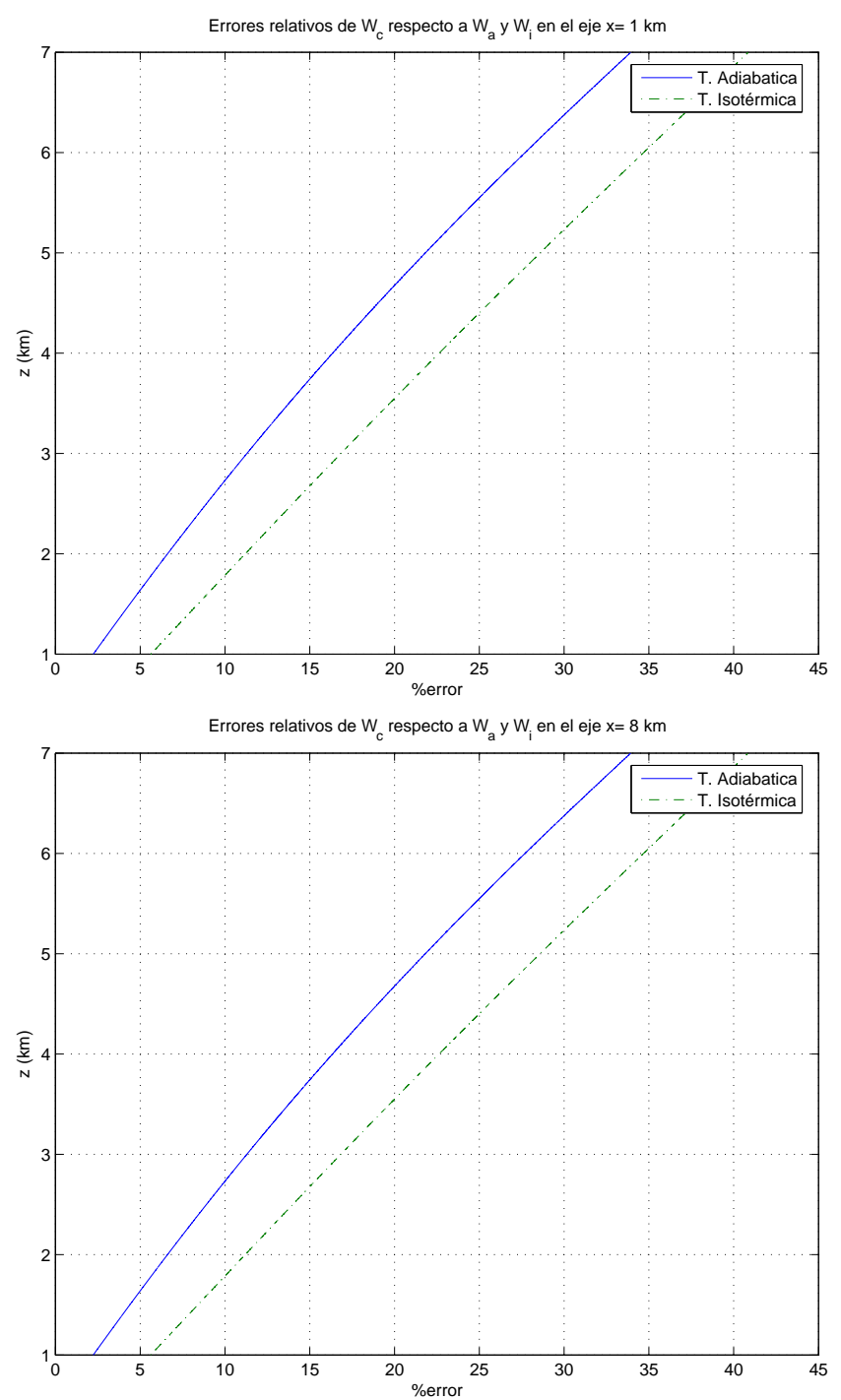

Figura 7.5: Errores relativos de la componente vertical del campo de vientos estimado con densidad constante respecto a la componente vertical del campo de vientos calculado con densidad dependiente de la coordenada vertical $z$ 


\section{Conclusiones y perspectivas}

En los dos primeros capítulos se han desarrollado las ecuaciones que gobiernan el movimiento de la atmósfera sobre la esfera terrestre, visto desde diferentes sistemas de coordenadas. También se ha calculado que la región de validez de las ecuaciones de momentum aproximadas es aproximadamente de $150 \mathrm{~km} \times 150 \mathrm{~km}$. Además, se ha demostrado con un argumento puramente geométrico que el modelo elipsoidal terrestre es más adecuado para estudiar la dinámica atmosférica, relegando al modelo esférico a un modelo de carácter local. Es por esta razón, que queda pendiente el planteamiento de todo la teoría desarrollada en este trabajo utilizando el modelo elipsoidal terrestre, con el fin de poder desarrollar modelos que realmente sean adecuados a escala global.

Se muestra que las variables atmosféricas (temperatura, presión, densidad, etc) pueden separarse en dos partes:

$$
\psi(\mathbf{r}, t)=\psi^{(0)}\left(z_{s}, t\right)+\sum_{k=1}^{\infty} \mu^{k} \psi^{k}(\mathbf{r}, t)
$$

Donde a $\psi^{(0)}(z, t)$ se le conoce como el estado de referencia atmosférico y a la suma $\sum_{k=1}^{\infty} \mu^{k} \psi^{k}(\mathbf{r}, t)$ se le conoce como la perturbación del estado de referencia. También se demostró, con un ejemplo numérico, que la definición (3.25) de tal estado de referencia es muy cercana al valor, en cada punto, de las variables atmosféricas calculadas con la ecuación de Bernoulli. Con dicho estado de referencia se simplificaron las ecuaciones desarrolladas anteriormente y, como resultado de dicha simplificación se obtuvo la ecuación de continuidad profunda la cual, es una de las restricciones utilizadas en el esquema variacional desarrollado en los capítulos 5-7. Con esto se da sentido práctico al presente trabajo pues, con las modificaciones mencionadas anteriormente, puede implementarse en un sistema de asimilación de datos de alguna institución dedicada a la meteorología. Queda pendiente explorar las implicaciones teóricas y prácticas que tiene la descomposición de las variables atmosféricas pues los términos $\mu^{k} \psi^{(k)}$ podrían ser de ayuda al modelar fenómenos como la turbulencia, etc.

El método de mapeo conforme para obtener un campo de velocidad analítico con divergencia cero resulto ser de gran utilidad. Nos evitó el problema de la obtención de datos reales del campo de viento además de darnos la oportunidad de verificar de manera confiable la bondad de esquema variacional. Ya que el campo que obtenemos con este método es bidimensional, queda pendiente su adaptación para obtener campos tridimensionales. Aunque el flujo alrededor de un cilindro es también útil para obtener datos sintéticos, el método de mapeo conforme nos da un campo con mayor complejidad y con él, se puede verificar de mejor manera la bondad del esquema variacional.

En el capítulo 5 se muestra el desarrollo del método variacional en un sistema cartesiano. Con este método se construye, en [30] y en [31], un campo de velocidad con divergencia cero a partir de los datos tomados del mapeo conforme desarrollado en el capítulo 4. Se muestra la discusión del efecto que tienen los parámetros $\alpha$ en el campo ajustado y con esto se demuestra que cualquier interpretación física o estadística de estos parámetros está fuera de lugar. Estos parámetros son sólo funciones de peso que ayudan a calibrar el ajuste del campo de velocidad. En [30] y en [31] se realizaron experimentos numéricos para poder determinar el valor óptimo de los parámetros $\alpha$, queda pendiente la creación de algún método que nos permita calcular automáticamente dichos parámetros utilizando los datos obtenidos en las redes de monitoreo ambiental.

Ya que el sistema de coordenadas cartesiano no es el más popular en el ambiente meteorológico, se planteó el método variacional para sistemas de coordenadas que siguen el terreno (coordenadas $\sigma$ ) y se obtienen las condiciones de frontera generalizadas que debe cumplir el multiplicador de Lagrange $\lambda$. También se analizan ejemplos reportados en la literatura poniendo especial interés en las condiciones de frontera que 
se utilizan. Se demuestra que en general son incorrectas y se dan ejemplos numéricos de ello.

Ante la duda de que la hipótesis de la atmósfera constante era lo mejor, en el último capítulo se desarrolla el método variacional para una atmósfera con densidad variable. Se comparan los campos obtenidos con ambas hipótesis y se observa la gran diferencia que hay. Por lo tanto, si se se desea obtener resultados más precisos, se debe tomar en cuenta la variación de la densidad con la altura.

A pesar de que la teoría aquí presentada aplica a casos tridimensionales, por simplicidad sólo se han dado ejemplos bidimensionales, quedando pendiente el desarrollo de los ejemplos tridimensionales. 


\section{Bibliografía}

[1] V. A Belinskii. Dynamic meteorology. Israel Program for Scientific Translations; available from the Office of Technical Services, U.S. Dept. of Commerce, Washington, 1961.

[2] J. C. Bernard, H. L. Wegley, and T.R. Hiester. Improving the performance of mass-consistent numerical models usin optimization tecniques. Journal Climate and Applied Meteorology, 26(6):675-686, 1987.

[3] Carl De Boor. A Practical Guide to Splines. Springer, 1 edition, 112001.

[4] S. S. Bunker C. G. Davis and J. P. Mutsclecner. Atmospheric transport models for complex terrain. Journal Climate Apllied Meteorology, pages 235-238, 1984.

[5] M. Caputo. The Gravity Field of the Earth. Academic Press New York, 1967.

[6] Kenneth R. Castleman. Digital Image Processing. Prentice Hall, 2r.e. edition, 81995.

[7] Philippe Ciarlet. The Finite Element Method for Elliptic Problems (Classics in Applied Mathematics). SIAM: Society for Industrial and Applied Mathematics, $2^{\circ}$ edition, 42002.

[8] Roger Daley. Atmospheric Data Analysis (CambridgeAtmospheric and Space Science Series). Cambridge University Press, 31991.

[9] Vivette Girault and Pierre-Arnaud Raviart. Finite Element Methods for Navier-Stokes Equations: Theory and Algorithms). Springer, 81986.

[10] Albert Gyr and Franz-S Rys, editors. Diffusion and Transport of Pollutants in Atmospheric Mesoscale Flow Fields (Ercoftac Series). Springer, 1 edition, 11995.

[11] Wolfgang Hackbusch. Elliptic Differential Equations: Theory and Numerical Treatment. Springer, 1 edition, 82003 .

[12] H. Hochstadt. Differential Equations: A modern Aproach. Dover New York, 1965.

[13] JamesR. Holton. An Introduction to Dynamic Meteorology, volume 88. Academic Press, fourth edition.

[14] Maarten Hooijberg. Practical Geodesy Using Computers. Springer, 1 edition, 101997.

[15] Toshihiro Kitada, Kiyomi Igarashi, and Michio Owada. Numerical analysis of air pollution in a combined field of land/sea and mountain/valley wind. Journal Climate and Applied Meteorology, 25:767-784, 1986.

[16] Lyle W. Konigsberg. Numerical Recipes Example Book (Fortran). Wayne State University Press, 41994.

[17] M. Krizek and P.Neittaanmaki. Finite Element Approximation of Variational Problems and Applications. Longman Sc and Tech, 71990.

[18] O. A. Ladyzhenskaya. The mathematical theory of viscous incompressible flow, volume 2 of Mathematics and its applications. GOrdon and Breach, Science plubishers, 2 edition, Marzo 1969.

[19] Demetri P. Lalas and Corrado F. Ratto, editors. Modelling of Atmospheric Flow Fields. World Scientific Pub Co Inc, 11996. 
[20] Rainald Lohner. Applied Computational Fluid Dynamics Techniques. John Wiley and Sons Inc 1/7/2001, 2001.

[21] G.C. McVitte. Quat. J. Mech. Appl. Math, 1:174-195, 1948.

[22] N. Moussiopoulus and Th. Flassak. Two vectorized algorithms for the effective calculations of massconsistent flow fields. Journal Climate and Applied Meteorology, 25(6):847-857, 1986.

[23] M. A. Núñez. Formal proof of the existence of an atmospheric base-state and its estimation. 11th Conference on Mesoscale Processes, Albuquerque, New Mexico, 2005. P1M.5.

[24] M. A. Núñez and E. Cruz. Analytic solutions of the deep continuity equation over a complex terrain. American Meteorological Society, 2005. P1M.13.

[25] M. A. Núñez, G. Ramírez, and H. Hernández. Análisis de métodos de interpolación de datos de vientos basados en la ecuación de conservación de la masa. Reporte de investigación, Universidad Autónoma Metropolitana, Abril 2004.

[26] M. A. Nuñez. Región de validez de modelos atmosféricos globales y su reformulación con un modelo elipsoidal terrestre. Reporte CBI No. 01.0106.I.011.02, Univ. Aut. Met. Izt., 2002.

[27] M. A. Nuñez. Región de validéz de algunos modelos atmosféricos de mesoescala. Reporte de Investigación UAM-I, CBI, 01.0106.01.009.2, Universidad Autónoma Metropolitana, 2002.

[28] M. A. Nuñez. Analysis of some atmospheric mesoscale models. Revista Mexicana de Fisica, 51(3):217, 2003.

[29] M. A. Núñez. Domain of validity of some atmospheric models. Il Nuovo Cimento C, 26(5):469-491, Sep-Oct 2003.

[30] M. A. Núñez, Ciro Flores, and Héctor Juárez. Interpolation of hydrodynamic velocity data with the continuity equation. Journal of Computational Methods in Sciences and Engineering, 3(2), 2006.

[31] M. A. Núñez, Ciro Flores, and Héctor Juárez. A study of hydrodynamic mass-consistent models. Journal of Computational Methods in Sciences and Engineering, 6(5-6):365-385, 2006.

[32] J. M. Ortega. Numerical Analysis. A second Course. Academic Press, New York, 1972.

[33] Roger A. Pielke. Mesoscale Meteorological Modeling(International Geophysics). Academic Press, 2 edition, 122001.

[34] Markus Raffel, Chris Willert, and J. Kompenhans. Particle Image Velocimetry: A Practical Guide (Experimental Fluid Mechanics). Springer, 1 edition, 62002.

[35] C. F. Ratto, R. Festa, C. Romero, O. A. Frumento, and M. Galluzzi. Mass consistent-models for wind fields over complex terrain: The state of the art. Environmental Software, 9:247-268, 1994.

[36] K. Rektorys. Variational Methods in Mathematics, Science and Engineering. Springer, 2 edition, 12 1899.

[37] Eduardo Rodríguez Barrera. Modelación y simulación numérica de campos de viento mediante elementos adaptativos en 3-D. Tesis doctoral, Universidad de las Palmas de Gran Canaria, Mayo 2004.

[38] D. G. Ross, I.Ñ. Smith., P. C. Manins, and D. G. Fox. Diagnostic wind field for complex terrain: Model developing and testing. Journal Apllied Meteorology, 30:785-796, 1988.

[39] C. A. Sherman. A mass consistent model for wind fields over complex terrain. Journal Applied Meteorology, 17:312-319, 1978 .

[40] Shang-Gyoo Shim, Seon-Yeon Cho, Gregory R. Charmichel, and T. Kitada. The sensivity of a 3dimensional eulerian model to unsertainties in emissions and dry deposition velocities. Atmospheric Environment, 20(10):1979-1987, 1986. 
[41] U.S. Geological Survey. Gtopo30, 1997. http://www.scd.ucar.edu/dss/datasets/ds758.0hmtl.

[42] Roger Temam. Navier-Stokes Equations, volume 2 of Studies in Mathematics and its applications. NorthHolland Publishing Company, Elsevier North-Holland, Inc. 52 Vanderbilt Avenue New York, NY 10017, reised edition edition, 1979 .

[43] H. J. Thiebaux and M.A. Pedder. Spatial Objective Analysis: WithApplications in Atmospheric Science. Academic Pr, 51987.

[44] M. Xue, K. K. Droegemeler, V. Wong, A. Shapiro, and K. Brewster. Advanced regional prediction system (arps), 1995. http://www.caps.ou.edu/ARPS. 


\title{
Universidad Autónoma Metropolitana \\ Unidad Iztapalapa \\ División de Ciencias Básicas e Ingeniería
}

\author{
Esquema Variacional para Estimar un Campo \\ de Velocidad Hidrodinámico a Partir de datos y \\ su Aplicación Meteorológica \\ Gerardo Ramírez Rosario \\ Maestría en Ciencias \\ Matemáticas Aplicadas e industriales
}

\author{
Asesores: \\ Nuiaz Penula Maras Antonio \\ Dr. Marco A. Núñez Peralta \\ Departamento de Física \\ Dr. Héctor Juárez Valencia \\ Departamento de Matemáticas
}

Sinodales:

Presidente:

Dr. Gustavo Izquierdo Buenrostro

Departamento de Matemáticas

Secretario:

Dr. Jorge E. Sánchez Sánchez

Laboratorio de Simulación de Materiales

Instituto de Matemáticas

Unidad Cuernavaca

\section{Vocal:}

Dra. María Luisa Sandoval Solís

Departamento de Matemáticas 